\title{
Arrival times in quantum mechanics: Operational and quantum optical approaches
}

\author{
Dissertation
}

zur Erlangung des Doktorgrades

der Mathematisch-Naturwissenschaftlichen Fakultäten der Georg-August-Universität zu Göttingen

vorgelegt von

Dirk Seidel

aus Halle(Saale)

Göttingen 2005 
D7

Referent: Prof. Dr. G.C. Hegerfeldt

Korreferent: Prof. Dr. K. Schönhammer

Tag der mündlichen Prüfung: 06.07.2005 


\section{Contents}

1 Introduction $\quad 1$

2 Arrival and dwell times in quantum mechanics 5

2.1 Arrival times . . . . . . . . . . . . . . . . 5

2.1.1 Classical arrival time . . . . . . . . . . . . . . . 5

2.1.2 Quantum arrival times for free motion . . . . . . . . . 7

2.1.3 Quantum mechanical flux . . . . . . . . . . . . . 8

2.1.4 Kijowski's distribution . . . . . . . . . . . . . . . 9

2.1.5 Aharonov-Bohm arrival-time operator and POVMs . . . . . . 10

2.1.6 Arrival-time distributions and the relation to local densities . 12

2.1.7 Phase times, tunneling times, transmission times . . . . . . 13

2.2 Dwell times . . . . . . . . . . . . . . . . . . . 15

2.2.1 Classical dwell time . . . . . . . . . . . . . . . . 15

2.2.2 Classical flux-flux correlation function . . . . . . . . . . . 16

2.2.3 Quantum dwell times . . . . . . . . . . . . . . . . . . 17

2.2.4 Quantum dwell-time operator . . . . . . . . . . . 18

2.2.5 Dwell-time distribution for free motion . . . . . . . . . . . 19

2.2.6 Moments of the dwell-time distribution . . . . . . . . . . . . 20

2.2.7 An alternative proposal for free dwell times . . . . . . . . 22

3 Operational approaches to quantum arrival times $\quad \mathbf{2 4}$

3.1 The quantum jump approach . . . . . . . . . . . . . 25

3.1.1 Conditional time evolution . . . . . . . . . . . . 25

3.1.2 The reset operation and the Bloch equation . . . . . . . 26

3.1.3 Exclusive and non-exclusive detection probability . . . . . . 28

3.1.4 Example: Two-level system with quantized $\mathrm{cm}$ motion . . . . 29

3.1.5 Corrections to the conditional time evolution . . . . . . . . . . 32

3.2 Laser-based approach to quantum arrival-time distributions . . . . . . 33

3.2.1 The probability density for the first photon . . . . . . . . . 35

3.2.2 Detection delay and reflection . . . . . . . . . . . . 37

3.2.3 Deconvolution and ideal distributions . . . . . . . . . . . . 39

3.2.4 Normalized arrival-time distributions . . . . . . . . . . . . 41

3.2.5 Galilean invariance of the first-photon distribution . . . . . . . 44

3.3 Arrival-time measurement with narrow laser fields . . . . . . . . . . . 45

3.3.1 The probability density for the first photon . . . . . . . . . 45 
3.3.2 Parameter regimes for the narrow laser model . . . . . . . . . 47

3.4 Arrival-time measurement with absorbing potentials . . . . . . . . . . 49

3.4.1 Absorbing potentials as a limiting case of the laser model . . . 50

3.4.2 Deconvolution of the absorption rate . . . . . . . . . . . 52

3.4.3 The reset state for absorption . . . . . . . . . . . . . . . 53

3.4.4 Position-dependent decay rate . . . . . . . . . . . . . . 54

3.5 Arrival-time distributions and relation to kinetic energy densities . . . 55

3.5.1 Local kinetic energy densities . . . . . . . . . . . . 55

3.5.2 Measurement-based approach to kinetic energy densities . . . 56

3.5.3 The case of fixed decay rate . . . . . . . . . . . . . 58

3.5.4 Expansion of Kijowski's distribution . . . . . . . . . . . 61

4 Operational approach to quantum dwell times $\quad \mathbf{6 3}$

4.1 Operational approach to mean dwell times . . . . . . . . . . . . . 63

4.2 Quantum dwell-time distributions and flux-flux correlation functions . 64

4.2.1 Quantum flux-flux correlations . . . . . . . . . . . . 64

4.2 .2 First moment . . . . . . . . . . . . . . . . 66 66

4.2 .3 Second moment . . . . . . . . . . . . . . . . 67

4.3 Operational approach to flux-flux correlation functions . . . . . . . . 69

4.3.1 Photon-photon correlations . . . . . . . . . . . . 69

5 Operator normalization $\quad 71$

5.1 Operator normalization of the quantum optical arrival-time model . . 71

5.1.1 Normalization on the level of operators . . . . . . . . . . 71

5.1 .2 Connection to Kijowski's arrival-time distribution . . . . . . . 73

5.1 .3 Fixed decay rate . . . . . . . . . . . . . . . 75

5.1.4 Operator normalization and connection with the flux . . . . 77

5.1.5 Alternative derivation of the operator normalization . . . . . 78

5.2 Operator normalization for narrow laser fields . . . . . . . . . . . 79

5.3 Operator normalization for absorbing potentials . . . . . . . . . 80

5.4 Measurement-based approaches to operator normalization . . . . . . . 82

5.4 .1 Operator normalization and filtering . . . . . . . . . . . 82

5.4.2 Real-valued filter potentials . . . . . . . . . . . . 84

6 Arrival times in the presence of interactions $\quad 93$

6.1 Operator-normalized absorption rate . . . . . . . . . . . . . . 94

6.2 Generalized free arrival-time distributions . . . . . . . . . . . . 96

6.2.1 Left incidence . . . . . . . . . . . . . . . . . . . . . . . . . . . . . . . .

6.2.2 Symmetric and antisymmetric incidence . . . . . . . . . . . 98

6.2.3 General incidence . . . . . . . . . . . . . . . . . 999

6.3 Generalized arrival-time distribution in the presence of interactions . 100

6.4 Example: Rectangular barrier . . . . . . . . . . . . . . . . . . . 101

6.4.1 Arrival time behind a potential barrier . . . . . . . . . . . . 101

6.4.2 Mean arrival times and the Hartman effect . . . . . . . . . . . 104

6.4.3 Arrival time inside a potential barrier . . . . . . . . . . . 107 
6.4.4 Arrival time in front of a potential barrier . . . . . . . . . 108

6.5 Example: Linear potentials . . . . . . . . . . . . . . . . . . . . 110

6.6 Example: Reflectionless potentials . . . . . . . . . . . . . . . 113

7 Conclusion 118

A Kijowski's axiomatic approach to arrival-time distributions $\quad \mathbf{1 2 2}$

A.1 The classical case . . . . . . . . . . . . . . . . . . . . . . . . 122

A.2 The quantum case . . . . . . . . . . . . . . . . 123

B Transfer matrix method $\quad \mathbf{1 2 5}$

B.1 One-channel case . . . . . . . . . . . . . . . . . 125

B.1.1 Potential barrier . . . . . . . . . . . . . 126

B.1.2 Dirac delta potential . . . . . . . . . . . . . . 127

B.1.3 Potential step . . . . . . . . . . . . . . . . . . 127

B.1.4 Continuous potentials . . . . . . . . . . . . . . 128

B.2 Two-channel case . . . . . . . . . . . . . . . . . . . . . . . . 129

B.2.1 Half-space laser field . . . . . . . . . . . . . . . . . 130

B.2.2 Laser barrier . . . . . . . . . . . . . . . . . . . 131

B.2.3 Dirac delta laser field . . . . . . . . . . . . . . . 131

C Faddeev-Marchenko inverse scattering methods $\quad 132$

C.1 One-dimensional scattering . . . . . . . . . . . . . . . . . . . 132

C.2 Relation between transmission and reflection coefficients . . . . . . 133

C.3 Marchenko equations without bound states . . . . . . . . . . . . . . 134

C.4 Marchenko equations with bound states . . . . . . . . . . . . 134

D Atomic units 135

$\begin{array}{ll}\text { Bibliography } & 135\end{array}$ 



\section{Chapter 1}

\section{Introduction}

In quantum mechanics, the notion of time arises in a twofold way. Firstly, time is a parameter appearing in the Schrödinger equation, which describes the evolution of states, but secondly it is an observable associated with measurable quantities such as lifetimes, arrival times, dwell times, etc. The theoretical analysis of several of these quantities has been controversial and is still subject to debate [1].

Within the last 10 years, a renewed interest in the treatment of such time observables has evolved. To some extent, this is due to the recent progress in quantum optics, nano-technology and in experimenting with single atoms at very low temperatures. This nowadays allows the observation and experimental confirmation of various effects and predictions associated with the topic of time in quantum mechanics. Against this background, it is very interesting and also necessary to design and to investigate operational, i.e. measurement-based models. The present work is devoted to this issue, with the main focus on operational approaches to quantum arrival times, although some results have been obtained for related quantities, as for instance dwell times and kinetic energy densities.

Time-of-flight measurements are frequently performed in experiments, but their outcome is mostly interpreted in a classical way, where the particles are assumed to be point-like, following defined trajectories [2]. This treatment is justified for fast particles, but not for slow ones, where the extension and spreading of the wave function may have to be taken into account and the concept of trajectories looses its meaning in an absolute sense. In this work, "slow" refers to atomic velocities of the order of $\mathrm{cm} / \mathrm{s}$, corresponding to temperatures of the order of $\mu \mathrm{K}$. If the spreading of the wave packet is large enough, it should result in an arrival-time distribution whose width is not negligible. Additionally, interference effects in the presence of external interactions may arise and completely change the outcome of an arrival-time measurement. In principle, these phenomena are observable today, for instance for slow atoms dropping out of a trap, but they are not well investigated from an experimental point of view. A noteworthy exception is the experiment of Szriftgiser et al. [3]. In contrast, arrival-time distributions for quantum particles are an extensively discussed topic in fundamental quantum physics [4]. This gap between experiment and theory has been deplored by several authors [1].

Historically, the peculiar role of time observables was noticed first in connection 
with the investigation of energy-time uncertainty relations and time operators. In 1933, Pauli [5] pointed out that the existence of a self-adjoint time operator canonically conjugate to the Hamiltonian implies the spectrum of the Hamiltonian to be extended over the whole axis and thus must be abandoned for systems with a bounded or semibounded energy spectrum. This has often been cited as the core of the conceptual difficulties.

Since then many attempts have been made to overcome Pauli's argument; here different research activities have to be distinguished. In the field of arrival times there are two main approaches: the first one is interested in an operational understanding of arrival times and arrival-time distributions without considering time operators. The principal problem here is the more or less abstract modeling of an experimental situation including "measurement devices" and to find appropriate limits in which the measured arrival times are independent of the specific properties of the device. In a series of pioneering papers, Allcock [6] investigated models which are based on the absorption rate in imaginary potentials, but he drew negative conclusions regarding a device-independent formulation. In contrast, Muga et al. constructed complex-valued potentials that perfectly absorb at a given wave number in an arbitrary small spatial interval [7]. Later on, Aharonov et al. [8] proposed several toy models for measuring arrival-time distributions and the authors noticed, in agreement with Allcock, that the distribution becomes distorted when the accuracy of the measurement is enhanced. In fact, this mirrors the key problem in an operational approach and has to be addressed in this work, too.

The second approach aspires to a satisfactory inclusion of time observables into the theory by defining and investigating ideal quantities for arrival-time operators, arrival-time distributions or average arrival times. Important milestones in this development and for free motion were achieved over the years. Aharonov and Bohm [9] introduced a time-of-arrival operator by a quantization of the classical expression which is not self-adjoint but maximally symmetric. Later, Kijowski [10] obtained an arrival-time distribution with minimal variance by imposing a set of intuitive axioms based on classical considerations. Finally, Giannitrapani [11] showed that the positive operator valued measure [12] associated with the Aharonov-Bohm time operator leads to Kijowski's distribution. This is the customary approach to arrival times of freely moving particles in the current literature.

The controversies grow with respect to arrival or dwell times in the presence of external interactions, since, unfortunately, the axiomatic treatment of Kijowski does not apply to the interaction case. The famous question "How long does it take for a particle to tunnel through a barrier?" has been widely discussed in the literature and various answers have been given, depending on the respective definition of tunneling time that has been chosen (for reviews, see Refs. [13, 14]). Particular interest has been attracted by papers that report "superluminal velocities" or "anomalously short traversal times", although these phenomena have been explained by pulsereshaping during the tunneling process and do not lead to violations of causality [15]. However, the discussion initiated recent efforts to measure tunneling times and related effects, at first with microwave pulses [16] or single-photon wave-packets [17]. The experimental results seem to identify the Hartman effect [18] and the 
Wigner time delay [19] as the physically relevant mechanisms for tunneling times and arrival times. Whether these results apply only to photons or can also be extended to other particles evolving with the Schrödinger equation is hard to decide for lack of experiments and a satisfactory theory of arrival-time distributions in the presence of interactions. It should be noted that anomalously short traversal times are no artifact of using the non-relativistic Schrödinger equation [20, 21].

As mentioned above, the current potentialities to experiment with single atoms at very low temperatures and to manipulate and prepare internal quantum states suggest modeling time-of-arrival measurements as realistically as possible to encourage experiments in this field. A step in this direction has been made by Halliwell [22], who put forward an effectively irreversible two-state detector coupled to the incoming particle, but without specifying a particular coupling mechanism. More recently, a quantum optical approach to arrival times has been proposed by Muga et al. [23] and has been investigated in detail in Ref. [24]. The idea of this model is to take the temporal distribution of the first spontaneous photon emitted by a twolevel atom which impinges on a spatially localized laser beam as an approximation of the arrival-time distribution.

In the present work, this quantum optical approach to arrival times is studied in detail and further developed. The aim is to investigate and to design operational models that can be used, at least in principle, to measure the various ideal quantities related to arrival-time distributions, as for example the quantum mechanical flux, Kijowski's distribution or the probability density of the wave packet times its mean velocity. Moreover, it will be shown that the first-photon approach provides a possibility to measure local kinetic energy densities and that it establishes a physically intuitive justification for models using absorbing potentials.

As a central result of this work, a first relation between an operational quantity and Kijowski's arrival-time distribution is found by means of the concept of operator normalization. This relation is used to generalize Kijowski's distribution to the case of particles interacting with some external potential. Generalizations like this have been widely discussed in the recent literature [4, 25], but they have not been proposed on an operational basis so far.

The organization of this work is as follows: in Chapter 2 an overview of some ideal quantities related to arrival times and dwell times is given. This provides the expressions that are used in this work as a reference for the operational models. Then, in Chapter 3, the quantum optical approach is introduced: in Section 3.1 the necessary theoretical description of the photon-emission probabilities of moving atoms is presented and in Section 3.2 the relation to arrival-times is established. The following sections of Chapter 3 are devoted to the investigation of various limiting cases of the model, in which some of the ideal quantities except for Kijowski's distribution are recovered. In particular, a first measurement-based approach to a positive quantum version of the local kinetic energy density is presented in Section 3.5. Chapter 4 presents some attempts to apply the quantum optical model to an operational approach of dwell-time distributions. A central result is the derivation of a new relation between the ideal dwell-time distribution which is based on the Ekstein-Siegert dwell-time operator [26] and quantum flux-flux correlation functions 
given in Section 4.2.

To relate the temporal first-photon probability density also to Kijowski's arrivaltime distribution, a new normalization procedure on the level of operators, recently proposed by Brunetti and Fredenhagen [27], is introduced in Chapter 5 and applied to the laser model. Again, various limiting cases are considered. It is shown in Section 5.4 that a measurement-based approach to the operator normalization can be given by means of a filtering procedure, where explicit filter potentials are derived.

Finally, in Chapter 6 arrival times in the presence of external potentials are considered. The operational first-photon approach is applied to that case and together with the formalism of operator normalization, a generalization of Kijowski's arrival-time distribution is obtained. This generalization is investigated for specific potentials and compared with previous approaches.

Appendix B introduces the notion of transfer matrices which provides a clear formalism for the derivation of scattering eigenfunctions, Appendix $\mathrm{C}$ presents a brief introduction into the one-dimensional inverse scattering methods that are employed in Section 5.4 and in Appendix D the atomic units used in this work are defined. 


\section{Chapter 2}

\section{Arrival and dwell times in quantum mechanics}

Two examples for time observables are arrival times and dwell or sojourn times. In the literature, they play an important role for the discussion and investigation of problems and peculiarities related to the concept of time in quantum mechanics (for recent reviews, see Refs. [1, 4]). The main focus of the present work is devoted to arrival times; only in Chapter 4 some results for dwell times are presented. Although arrival times and dwell times are very much related in classical mechanics, their quantum mechanical counterparts are in general different. In this chapter, a brief overview of basic concepts for arrival times and dwell times in classical and quantum mechanics is given. Particular emphasis is placed on the different definitions and proposals of the corresponding distributions.

The expressions reviewed in this chapter are referred to as "ideal" within this work. This means that they rely only on theoretical considerations without any relations to actual experiments or measurement situations. In contrast, the following chapters of this work are devoted to an operational (measurement-based) understanding of arrival-time distributions and dwell-time distributions. In this connection, the ideal quantities may serve as a reference for comparison with the operational quantities.

\subsection{Arrival times}

\subsubsection{Classical arrival time}

The understanding of arrival-time distributions for an ensemble of classical particles is fundamental for considering quantum versions. Although the notion of the free classical arrival time is straightforward, the case of interacting particles and related multiple crossings is problematic even in classical mechanics. In this work, only deterministic motion represented by Liouvilles's equation is considered. A discussion of stochastic Brownian motion and corresponding references can be found in Ref. [4]. 
Free motion: In classical mechanics and for free motion, the arrival time at $x=$ $x_{\mathrm{A}}$ of a particle with initial position $x_{0}<x_{\mathrm{A}}$ and initial momentum $p_{0}>0$ is given by

$$
t_{\mathrm{A}}=\frac{m\left(x_{\mathrm{A}}-x_{0}\right)}{p_{0}}
$$

Since the particle crosses the point $x_{\mathrm{A}}$ once and only once, Eq. (2.1) also provides the first-arrival time. The distribution of these times for a classical ensemble described by the phase-space distribution function $\varrho_{t}(x, p)$ with $\varrho_{t}(x, p \leq 0)=0$ is given by the classical probability flux or current density $J^{\mathrm{cl}}(t, x)$,

$$
J^{\mathrm{cl}}\left(t, x_{\mathrm{A}}\right)=\int_{-\infty}^{\infty} \mathrm{d} x \int_{0}^{\infty} \mathrm{d} p \varrho_{t}(x, p) \frac{p}{m} \delta\left(x-x_{\mathrm{A}}\right)
$$

which is the average of the phase-space function

$$
\mathcal{J}\left(x, p ; x_{\mathrm{A}}\right)=\frac{p}{m} \delta\left(x-x_{\mathrm{A}}\right)
$$

The average free arrival time, $\left\langle t_{\mathrm{A}}\right\rangle$, for the distribution (2.2) is given by the first moment, $\int_{-\infty}^{\infty} \mathrm{d} t t J^{\mathrm{cl}}\left(t, x_{\mathrm{A}}\right)$, which becomes with Liouville's theorem and $x(t)=$ $x_{0}+p_{0} t / m$

$$
\left\langle t_{\mathrm{A}}\right\rangle=\int_{-\infty}^{\infty} \mathrm{d} x_{0} \int_{0}^{\infty} \mathrm{d} p_{0} \varrho_{0}\left(x_{0}, p_{0}\right) \frac{m\left(x_{\mathrm{A}}-x_{0}\right)}{p_{0}},
$$

i.e. the average of the times $t_{\mathrm{A}}$ with respect to the phase-space distribution function.

For an ensemble of free particles with positive and negative momenta, the sum of the positive flux, given by

$$
J_{+}^{\mathrm{cl}}\left(t, x_{\mathrm{A}}\right)=\int_{0}^{\infty} \mathrm{d} p \varrho_{t}\left(x_{\mathrm{A}}, p\right) \frac{p}{m}
$$

and minus the negative flux, given by

$$
J_{-}^{\mathrm{cl}}\left(t, x_{\mathrm{A}}\right)=\int_{-\infty}^{0} \mathrm{~d} p \varrho_{t}\left(x_{\mathrm{A}}, p\right) \frac{p}{m}
$$

is the total arrival-time distribution [4],

$$
J_{\text {tot }}^{\mathrm{cl}}\left(t, x_{\mathrm{A}}\right)=J_{+}^{\mathrm{cl}}\left(t, x_{\mathrm{A}}\right)-J_{-}^{\mathrm{cl}}\left(t, x_{\mathrm{A}}\right)=\int_{-\infty}^{\infty} \mathrm{d} x \int_{-\infty}^{\infty} \mathrm{d} p \varrho_{t}(x, p) \frac{|p|}{m} \delta\left(x-x_{\mathrm{A}}\right) .
$$

This corresponds to the ensemble average of the phase space function

$$
\mathcal{J}_{\text {tot }}^{\text {cl }}\left(x, p ; x_{\mathrm{A}}\right)=\frac{|p|}{m} \delta\left(x-x_{\mathrm{A}}\right)
$$


Interaction case: For classical particles interacting with some external potential, multiple crossings or no crossings of the arrival point $x_{\mathrm{A}}$ are possible. In that case, the arrival-time distribution $J_{\text {tot }}^{\text {cl }}\left(t, x_{\mathrm{A}}\right)$ of Eq. $(2.7)$ is no longer a first-passage distribution, since no distinction is made between first, second or $n$th arrivals. As a consequence, $J_{\text {tot }}^{\mathrm{cl}}\left(t, x_{\mathrm{A}}\right)$ is in general not normalized and even not normalizable, for instance for a particle oscillating in a harmonic potential. It can therefore not be considered as a probability distribution, but it has nevertheless a physical meaning since the ratio of two values for two different times gives the relative number of arrivals for those times [4].

As a proposal for a first-passage distribution several authors consider the absorption rate of particles which are eliminated when crossing $x=x_{\mathrm{A}}$ due to absorbing boundary conditions [4]. This boundary condition is modeled by $\lim _{\epsilon \rightarrow 0+} \varrho_{t}\left(x_{\mathrm{A}}-\right.$ $\epsilon, p)=0$ for $p<0$, and the corresponding expression for the absorption rate is given by

$$
J_{\text {abs }}^{\mathrm{cl}}\left(t, x_{\mathrm{A}}\right)=-\frac{\mathrm{d} N^{\mathrm{cl}}(t)}{\mathrm{d} t},
$$

where $N^{\mathrm{cl}}(t)=\int_{-\infty}^{\infty} \mathrm{d} q \int_{-\infty}^{\infty} \mathrm{d} p \varrho_{t}(q, p)$ is the time-dependent decreasing norm which depends on $x_{\mathrm{A}}$. If not all particles reach the arrival point $x_{\mathrm{A}}, J_{\text {abs }}^{\mathrm{cl}}\left(t, x_{\mathrm{A}}\right)$ is not normalized to 1 and it can be normalized by dividing Eq. (2.9) by the total norm absorbed, $\int \mathrm{d} t J_{\text {abs }}^{\mathrm{cl}}\left(t, x_{\mathrm{A}}\right)=1-N^{\mathrm{cl}}(\infty)$.

\subsubsection{Quantum arrival times for free motion}

The introduction of the time-of-arrival as a quantum observable into the standard theory has a long history. Various proposals and suggestions for this quantity have been presented and controversially discussed. In this connection, one has to distinguish clearly between efforts that have been undertaken to define a time-of-arrival operator and those which deal with the definition of an average arrival time or an arrival-time distribution for quantum particles. In this work, the latter subject is solely investigated. Moreover, difficulties increase when considering traversal or tunneling times in the presence of external potentials. The interaction case is briefly introduced in Section 6, whereas concepts for free arrival times are summarized in the following. Throughout this work, the hat $\widehat{\wedge}$ is used to distinguish operators from corresponding c-numbers.

A first negative result concerning a general time operator in quantum mechanics is due to Pauli [5]. He showed that the existence of a self-adjoint operator $\widehat{T}$ which is canonically conjugate to the Hamiltonian,

$$
[H, \widehat{T}]=\mathrm{i} \hbar
$$

would allow the application of the unitary operator $\mathrm{e}^{-\mathrm{i} E^{\prime} \widehat{T} / \hbar}$ to the energy eigenstate $|E\rangle$, which yields another energy eigenstate $\left|E-E^{\prime}\right\rangle$ with eigenvalue $E-E^{\prime}$. This means that the spectrum of $H$ would extend over the range $[-\infty, \infty]$, which forbids the existence of $\widehat{T}$ for Hamiltonians with bounded or semibounded energy spectrum.

In spite of this argument, many attempts have been made to overcome this problem for the case of arrival times. An important contribution is due to Aharonov 
Chapter 2. Arrival and dwell times in quantum mechanics

and Bohm [9], who introduced a non-self-adjoint time-of-arrival operator $\widehat{T}_{\mathrm{AB}}$ by quantization and symmetrization of the classical expression (2.1). This is briefly reviewed in Section 2.1.5. Grot et al. constructed a self-adjoint variant of $\widehat{T}_{\mathrm{AB}}$ which circumvents Pauli's argument by modifying the commutator relation (2.10) [28]. Other proposals have been presented by Razavi [29], Kijowski [10] and by Delgado and Muga [30]. An interesting recent approach is due to Galapon [31, 32], who considered self-adjoint time-of-arrival operators for spatially confined, but otherwise free, particles.

On the other hand, many authors attempted to find an arrival-time distribution for free particles without defining a proper time-operator. In a pioneering work [6], Allcock studied a time-of-arrival distribution that is based on a simplified detection procedure by means of an absorbing potential. He pessimistically concluded that a detector-independent formulation cannot be found, but he proposed to obtain an ideal arrival-time distribution by a deconvolution of the absorption rate with the apparatus response. Later, Kijowski [10, 33] presented an axiomatic approach to free arrival times which in the classical case recovers the distribution (2.2). Applying the axioms to the quantum case, he obtained a positive arrival-time distribution referred to as Kijowski's distribution in the following (see Section 2.1.4). A more general treatment which is based on Kijowski's work has been presented by Werner [34]. Further contributions to arrival-time distributions are due to Yamada and Takaki $[35,36,37]$ in the framework of consistent histories and Feynman path integral formalism and due to Kochanski and Wodkiewicz [38]. A treatment in the framework of Bohmian mechanics has been put forward by Leavens [39, 40] and other authors [41]. Moreover, Ruseckas and Kaulakys recently used the concept of weak measurements to define arrival-time distributions [42, 43].

An important step towards a satisfactory theory has been made by Giannitrapani [11], who showed that the arrival-time operator obtained by Aharonov and Bohm leads to Kijowski's distribution by means of the concept of positive operator valued measures (POVMs). POVMs consistently generalize standard quantum mechanics and in particular the usual projection-valued measures [12]. This is nowadays a widely accepted formalism for arrival-time distributions, and further progress in this direction has been obtained [44, 45, 46, 47].

Recent reviews about the subject of arrival times in quantum mechanics can be found in Refs. [1, 4].

\subsubsection{Quantum mechanical flux}

In classical mechanics, the current density or probability flux $J^{\mathrm{cl}}(t, x)$ of Eq. (2.2) has been defined as the arrival-time distribution for particles with positive momenta. It is obvious to apply the same definition in quantum mechanics, i.e. to take the quantum mechanical flux $J(t, x)$ which is given by

$$
J(t, x)=\frac{\hbar}{m} \operatorname{Im}\left(\overline{\psi(x, t)} \frac{\partial}{\partial x} \psi(x, t)\right),
$$

as a distribution for the time-of-arrival of the state $\left|\psi_{t}\right\rangle$. The drawback of this approach is the so-called backflow effect, which means that for a freely evolving 
quantum particle with nonzero wave number components only for $k>0$ it is possible for $J(t, x)$ to be negative over an arbitrary long, but finite, time interval [48]. A first mention of this effect can be found in Ref. [6]. This non-positiveness of the quantum mechanical flux prevents an interpretation as a true probability distribution for arrival times. Example wave packets showing the backflow effect are given in this work, see e.g. Fig. 3.4. Nevertheless, the flux is an easily calculable quantity that often provides a well-defined expression for an arrival-time distribution.

More difficulties arise when one considers the general classical result (2.7). Since $J_{+}^{\text {cl }}$ and $J_{-}^{\text {cl }}$ of Eqs. (2.5) and (2.6) are phase-space averages of the functions $\mathcal{J}_{+}(q, p)=$ $\delta\left(q-x_{\mathrm{A}}\right) \frac{p}{m} \Theta(p)$ and $\mathcal{J}_{-}(q, p)=\delta\left(q-x_{\mathrm{A}}\right) \frac{p}{m} \Theta(-p)$, a quantization of these expressions to obtain the corresponding one-sided quantum flux operators is non-unique since the flux operator does not commute with $\Theta( \pm \widehat{p})$ and there are infinitely many symmetrization possibilities [4]. Paradoxically, many of the "positive flux" operators obtained in this manner are not positive definite due to the backflow effect and even the classical decomposition $J_{\text {tot }}=J_{+}-J_{-}$is not preserved in the quantum case.

In the Bohm trajectory approach of quantum mechanics, the positive expression

$$
J_{\mathrm{B}}\left(t, x_{\mathrm{A}}\right)=\frac{\left|J\left(t, x_{\mathrm{A}}\right)\right|}{\int_{-\infty}^{\infty} \mathrm{d} t\left|J\left(t, x_{\mathrm{A}}\right)\right|}
$$

is obtained for the distribution of arrival times for those particles that actually reach $x_{\mathrm{A}}$ [39]. In this framework, the peculiarities concerning a decomposition into right and left incoming flux components are absent, but it is not clear how to justify the expression (2.12) in standard quantum mechanics.

\subsubsection{Kijowski's distribution}

In an important paper for the field of time in quantum mechanics, Kijowski introduced axoims for an arrival-time distribution for free motion arrivals at $x_{\mathrm{A}}$ that is based on classical correspondence [10, 33]. His approach is reviewed in Appendix A. For free particles with only positive momentum components, the arrival-time distribution of Kijowski at $x=x_{\mathrm{A}}$ can be written as

$$
\begin{aligned}
\Pi_{\mathrm{K}}\left(t, x_{\mathrm{A}}\right) & =\left\langle\psi_{t}\left|\frac{1}{m} \widehat{p}^{1 / 2} \delta\left(\widehat{x}-x_{\mathrm{A}}\right) \widehat{p}^{1 / 2}\right| \psi_{t}\right\rangle \\
& =\frac{\hbar}{2 \pi m}\left|\int_{0}^{\infty} \mathrm{d} k \widetilde{\psi}(k) \sqrt{k} \mathrm{e}^{-\mathrm{i} \hbar k^{2} t / 2 m} \mathrm{e}^{\mathrm{i} k x_{\mathrm{A}}}\right|^{2} .
\end{aligned}
$$

The operator in Eq. (2.13) is easily understood as a positive quantization of the classical expression (2.3), but its derivation is based on an axiomatic treatment (see Appendix A). For positive momentum components, $\Pi_{\mathrm{K}}\left(t, x_{\mathrm{A}}\right)$ is the same as the distribution that has been obtained later in terms of positive operator valued measures (POVMs), as shown in Section 2.1.5. The covariance of $\Pi_{\mathrm{K}}\left(t, x_{\mathrm{A}}\right)$ under time translations,

$$
\Pi_{\mathrm{K}}\left(t, x_{\mathrm{A}} ; \psi\left(t_{0}\right)\right)=\Pi_{\mathrm{K}}\left(t+t_{0}, x_{\mathrm{A}} ; \psi(0)\right)
$$


has been emphasized by Werner [49] as one of the basic properties of Kijowski's distribution.

Note that both the flux $J\left(t, x_{\mathrm{A}}\right)$ and Kijowski's distribution $\Pi_{\mathrm{K}}\left(t, x_{\mathrm{A}}\right)$ can be written in an uniform manner in $k$-space by defining kernel functions $f_{J}\left(k, k^{\prime}\right)=$ $\left(k+k^{\prime}\right) / 2$ and $f_{\mathrm{K}}\left(k, k^{\prime}\right)=\sqrt{k k^{\prime}}$, i.e. the arithmetic and geometric mean of $k$ and $k^{\prime}$, and by rewriting Eqs. (2.11) and (2.14) in the following form:

$$
\begin{aligned}
J\left(t, x_{\mathrm{A}}\right) & =\frac{\hbar}{2 \pi m} \int_{0}^{\infty} \mathrm{d} k \mathrm{~d} k^{\prime} \overline{\widetilde{\psi}(k)} \widetilde{\psi}\left(k^{\prime}\right) \mathrm{e}^{\mathrm{i} \hbar\left(k^{2}-k^{\prime 2}\right) t / 2 m} \mathrm{e}^{-\mathrm{i}\left(k-k^{\prime}\right) x_{\mathrm{A}}} f_{J}\left(k, k^{\prime}\right) \\
\Pi_{\mathrm{K}}\left(t, x_{\mathrm{A}}\right) & =\frac{\hbar}{2 \pi m} \int_{0}^{\infty} \mathrm{d} k \mathrm{~d} k^{\prime} \overline{\widetilde{\psi}(k)} \widetilde{\psi}\left(k^{\prime}\right) \mathrm{e}^{\mathrm{i} \hbar\left(k^{2}-k^{\prime 2}\right) t / 2 m} \mathrm{e}^{-\mathrm{i}\left(k-k^{\prime}\right) x_{\mathrm{A}}} f_{\mathrm{K}}\left(k, k^{\prime}\right) .
\end{aligned}
$$

From this, one easily checks that the first moment of both distributions agree,

$$
\langle t\rangle=\frac{m}{\hbar} \int_{0}^{\infty} \mathrm{d} k|\widetilde{\psi}(k)|^{2} \frac{1}{k}\left(x_{\mathrm{A}}+\frac{\partial}{\partial k} \arg \widetilde{\psi}(k)\right)=\int_{-\infty}^{\infty} \mathrm{d} t t J\left(t, x_{\mathrm{A}}\right)=\int_{-\infty}^{\infty} \mathrm{d} t t \Pi_{\mathrm{K}}\left(t, x_{\mathrm{A}}\right),
$$

where $\widetilde{\psi}(k)=|\widetilde{\psi}(k)| \exp (\mathrm{i} \arg \widetilde{\psi}(k))$. In this work, Gaussian wave packets are considered for all numerical examples, for which one has

$$
\widetilde{\psi}(k)=\frac{1}{\sqrt{\Delta k \sqrt{2 \pi}}} \mathrm{e}^{-\left(k-k_{0}\right)^{2} /\left(4 \Delta k^{2}\right)} \mathrm{e}^{-\mathrm{i} k x_{0}},
$$

where $x_{0}$ is the mean position, $k_{0}$ is the mean momentum and $\Delta k$ the momentum spread at $t=0$. From Eq. (2.19) it follows that $\frac{\partial}{\partial k} \arg \widetilde{\psi}(k)=-x_{0}$. Inserting this expression into Eq. (2.18) leads to

$$
\langle t\rangle=\frac{m}{\hbar} \int_{0}^{\infty} \mathrm{d} k|\widetilde{\psi}(k)|^{2} \frac{1}{k}\left(x_{\mathrm{A}}-x_{0}\right),
$$

which is the quantum average of the classical arrival time $t_{\mathrm{A}}$ of Eq. (2.1).

For the interacting case and also for general momentum components, $p \lessgtr 0$, Kijowski's axiomatic approach does not seem to be directly applicable and generalizations for particles in the presence of external potentials are still objects of research $[25,50]$. This is discussed more extensively in Chapter 6 , where a generalized arrival-time distribution is derived by means of the new operational approach given in this work.

\subsubsection{Aharonov-Bohm arrival-time operator and POVMs}

For the introduction of a "clock" which measures time using the position and momentum of a freely moving test particle, Aharonov and Bohm [9] introduced a nonself-adjoint time-of-arrival operator $\widehat{T}_{\mathrm{AB}}$ by a quantization of the classical expression (2.1). This operator is given by

$$
\widehat{T}_{\mathrm{AB}}=-\frac{m}{2}\left(\left(\widehat{x}-x_{\mathrm{A}}\right) \widehat{p}^{-1}+\widehat{p}^{-1}\left(\widehat{x}-x_{\mathrm{A}}\right)\right),
$$


and it has been extensively investigated in the literature [11, 12, 44, 47]. In the following, some useful results for this work are summarized.

The time-of-arrival operator $\widehat{T}_{\mathrm{AB}}$ of Eq. (2.21) is not self-adjoint but maximally symmetric $^{1}$. It provides the correct commutation relation with the free Hamiltonian, $H=\widehat{p}^{2} / 2 m$, namely

$$
\left[H, \widehat{T}_{\mathrm{AB}}\right]=\mathrm{i} \hbar \text {. }
$$

Moreover, $\widehat{T}_{\mathrm{AB}}$ has a domain which is given by the functions that in momentum representation vanish at $p=0$ according to $\lim _{p \rightarrow 0} \tilde{\phi}(p) p^{-3 / 2} \rightarrow 0$. The weak eigenvectors of $\widehat{T}_{\mathrm{AB}}$ for the eigenvalue $t$ are twofold degenerated and they are given by

$$
\langle k \mid t, \pm\rangle=\sqrt{\frac{\hbar|k|}{2 \pi m}} \mathrm{e}^{\mathrm{i} \hbar k^{2} t / 2 m} \mathrm{e}^{-\mathrm{i} k x_{\mathrm{A}}} \Theta( \pm k),
$$

where $|k\rangle$ are the eigenstates of the operator $\widehat{k}=\widehat{p} / \hbar$. The states $|t, \pm\rangle$ are not orthogonal, since

$$
\left\langle t^{\prime}, \alpha^{\prime} \mid t, \alpha\right\rangle=\frac{\delta_{\alpha, \alpha^{\prime}}}{2}\left(\delta\left(t-t^{\prime}\right)+\frac{\mathrm{i}}{\pi} \mathcal{P} \frac{1}{t-t^{\prime}}\right), \quad \alpha= \pm, \alpha^{\prime}= \pm,
$$

but they are complete and they provide a resolution of the unity,

$$
\widehat{1}=\sum_{ \pm} \int_{-\infty}^{\infty} \mathrm{d} t|t, \pm\rangle\langle t, \pm| .
$$

This allows to define an arrival-time distribution in terms of a positive operator valued measure (POVM) which is a mapping from intervals on the real line to positive operators which add together for disjoint intervals and which add to unity when the intervals are summed over the real line [12]. POVMs provide a generalization of standard quantum mechanics, where observables correspond to self-adjoint operators and probabilities are derived by the expectation values of the corresponding projection operators. The theory of POVMs shows that for the definition of measurement probabilities it is sufficient to work with the eigenstates of a maximally symmetric operator, as $\widehat{T}_{\mathrm{AB}}$. In the case of arrival times, the eigenstates (2.23) define a POVM given by

$$
\widehat{\Pi}\left(t_{2}, t_{1}\right)=\sum_{ \pm} \int_{t_{1}}^{t_{2}} \mathrm{~d} t|t, \pm\rangle\langle t, \pm|,
$$

i.e. the probability for the arrival time of a state $|\psi\rangle$ to be between $t_{1}$ and $t_{2}$ is given by $\left\langle\widehat{\Pi}\left(t_{2}, t_{1}\right)\right\rangle_{\psi}$, and the probability distribution for arrival-times thus reads [11]

$$
\begin{aligned}
\Pi_{\mathrm{POVM}}(t) & =\left\langle\psi\left|\left(\sum_{ \pm}|t, \pm\rangle\langle t, \pm|\right)\right| \psi\right\rangle=\sum_{ \pm}|\langle t, \pm \mid \psi\rangle|^{2} . \\
& =\frac{\hbar}{2 \pi m} \sum_{ \pm}\left|\int_{0}^{\infty} \mathrm{d} k \widetilde{\psi}( \pm k) \sqrt{k} \mathrm{e}^{-\mathrm{i} \hbar k^{2} t / 2 m} \mathrm{e}^{ \pm \mathrm{i} k x_{\mathrm{A}}}\right|^{2} .
\end{aligned}
$$

\footnotetext{
${ }^{1}$ In fact, the deficiency indices are $(2,0)$. This means that the adjoint of $\widehat{T}_{\mathrm{AB}}$ has two normalizable eigenstates with eigenvalue $\mathrm{i}$ and none with eigenvalue $-\mathrm{i}$ [47].
} 
Chapter 2. Arrival and dwell times in quantum mechanics

This distribution is positive, in contrast to the quantum mechanical flux. For only positive momenta, $\Pi_{\mathrm{POVM}}(t)$ coincides with Kijowski's distribution given in Section 2.1.4.

\subsubsection{Arrival-time distributions and the relation to local densities}

Arrival-time distributions can be understood as quantum local densities which provides a concept that sheds new light on some peculiarities concerning different quantum versions of the classical flux [51, 52].

To obtain an expression for the local density of a quantum observable not diagonal in coordinate representation, one may look for guidance to the corresponding classical case. The local density $\alpha_{A}^{\mathrm{cl}}(x)$ for a classical dynamical variable $A(q, p)$ is obtained with the phase-space density $\varrho_{t}(q, p)$ in terms of a phase-space integral,

$$
\alpha_{A}^{\mathrm{cl}}(x, t)=\int \mathrm{d} q \int \mathrm{d} p \varrho_{t}(q, p) A(q, p) \delta(q-x) .
$$

Clearly, integration over $x$ yields the average value of $A(q, p), \int \mathrm{d} x \alpha_{A}(x, t)=\langle A\rangle_{t}$. To quantize the expression for $\alpha_{A}(x)$, the operator identity

$$
\delta(\widehat{x}-x)=|x\rangle\langle x|
$$

is used, and the operator $\widehat{A}(x)=\widehat{A}|x\rangle\langle x|$, or rather one of its symmetrizations, is considered as a quantum density operator for the observable $\widehat{A}$. The expectation value of $\widehat{A}(x)$ consequently yields the value of the quantum local density at the point $x$,

$$
\alpha_{A}(x, t)=\left\langle\psi_{t}|\widehat{A}(x)| \psi_{t}\right\rangle .
$$

If $\widehat{A}$ is not diagonal in coordinate representation, it does not commute with $|x\rangle\langle x|$, and they are infinitely many symmetrizations to construct $\widehat{A}(x)$ and therefore local quantum densities in general, for example

$$
\begin{aligned}
\widehat{A}(x) & =\widehat{A}^{1 / 2}|x\rangle\langle x| \widehat{A}^{1 / 2}, \\
\text { or } \widehat{A}(x) & =\frac{1}{2}(\widehat{A}|x\rangle\langle x|+| x\rangle\langle x| \widehat{A}), \\
\text { or } \widehat{A}(x) & =\frac{1}{2} \widehat{A}^{1 / 2}|x\rangle\langle x| \widehat{A}^{1 / 2}+\frac{1}{4}(\widehat{A}|x\rangle\langle x|+| x\rangle\langle x| \widehat{A}) .
\end{aligned}
$$

The non-commutativity of two observables does not mean that there is only one "true" symmetrization of their product. Different symmetrizations may have a perfectly respectful status as physically observable and measurable quantities, and different orderings may be associated with latent properties that may be realized via different experimental measurement procedures. They may also be related more indirectly to observables and yet carry valuable physical information. In fact it can be argued that only measurement procedures can give an answer to the question, 
which distribution is physically meaningful. For the case of arrival-time distributions, which are nothing more than local velocity densities, this question plays the central role in the present work.

Note that the first symmetrization of $\widehat{A}(x)$ in Eq. (2.32) provides a positive version of a quantum density, whereas the others may become negative for some values of $x$ and $t$.

The classical expression that has to be symmetrized for a velocity density is given by Eq. (2.3). The expectation values of the operators

$$
\begin{aligned}
& \widehat{J}_{1}(x)=\frac{1}{m} \widehat{p}^{1 / 2}|x\rangle\langle x| \widehat{p}^{1 / 2}, \\
& \widehat{J}_{2}(x)=\frac{1}{2 m}(\widehat{p}|x\rangle\langle x|+| x\rangle\langle x| \widehat{p})
\end{aligned}
$$

are easily shown to yield Kijowski's distribution $\Pi_{\mathrm{K}}(t, x)$ and the quantum mechanical flux $J(t, x)$, respectively:

$$
\begin{aligned}
\Pi_{\mathrm{K}}(t, x) & =\frac{1}{m}\left\langle\psi_{t}\left|\widehat{p}^{1 / 2}\right| x\right\rangle\left\langle x\left|\widehat{p}^{1 / 2}\right| \psi_{t}\right\rangle, \\
J(t, x) & =\frac{1}{2 m}\left\langle\psi_{t}|(\widehat{p}|x\rangle\langle x|+| x\rangle\langle x| \widehat{p})| \psi_{t}\right\rangle .
\end{aligned}
$$

In Eqs. (2.35) and (2.37) it is assumed that $\left|\psi_{t}\right\rangle$ has only support for positive momenta such that $\widehat{p}^{1 / 2}$ is well defined on these states. Notice that the quantization rule that yields the flux $J(t, x)$ is the Weyl-Wigner rule [46]. Again, the symmetrization that leads to $\Pi_{K}(t, x)$ is by construction positive, whereas the flux may become negative and exhibits the backflow effect.

The justification of Eqs. (2.38) and (2.37) in terms of measurement-based procedures is the central issue of this work. For the flux $J(t, x)$, operational procedures have been proposed in Ref. [24] and they are explained in Section 3.2, whereas for Kijowski's distribution a first operational understanding is obtained in Chapter 5 of this work.

Of course, the discussion of local quantum densities is not restricted to velocity densities. Another example with considerable importance in chemical physics are local kinetic energy densities, for which an operational approach is given in Section 3.5.

\subsubsection{Phase times, tunneling times, transmission times}

As pointed out in Section 2.1.2, difficulties and controversies grow when considering arrival times in the presence of interactions. The famous question "How long does it take for a particle to cross a potential barrier?" has been a long discussed issue in the past and it has recently attracted renewed attention due to the progress in nano-technology [53] and in photonic and microwave tunneling experiments [54, 55, 56]. This question is fundamentally intertwined with the question of dwell times considered in Section 2.2.

This section is devoted to the introduction of a few expressions and equations that will become important in this work and for an overview of references. It is not 
intended to cover the whole field, reviews on the subject of phase times, tunneling times, transmission times and related topics can be found in Refs. [1, 13, 57, 14].

As one of the first contributions, Wigner [19] introduced the notion of phase times by considering the peaks of incoming and transmitted wave packets within the stationary phase approximation, and for the tunneling time through a potential region of width $L=x_{2}-x_{1}, x_{1}<x_{2}$, with the corresponding tunneling amplitude denoted by $T(k)$ he obtained

$$
t_{\mathrm{ph}}(k)=\frac{m}{\hbar k}\left(L+\frac{\partial}{\partial k} \arg T(k)\right) .
$$

This equation holds for nearly monochromatic waves, where $m L /(\hbar k)$ is the time the freely moving packet would need to cross the region and thus $m \frac{\partial}{\partial k}(\arg T(k)) /(\hbar k)$ is the time delay associated with the transmission. These phase times are the basis for the exploration of the Hartman effect for potential barriers [18, 58, 59, 60] which says that for an opaque barrier there are regimes where the tunneling time becomes essentially independent of the width $L$ of the barrier region (for more details, see Section 6.4.2).

Phase times have become a source of confusion due to its asymptotic character and due to the fact that the peak of the wave packet is not a reliable characteristic of packets distorted by tunneling [13]. In fact, it has been pointed out that no physical law guarantees that an incoming peak turns into an outgoing peak [61].

More recently, transmission (reflection) times $\tau_{\mathrm{T}, \mathrm{R}}$ have been introduced by means of the difference of the average incoming and transmitted (reflected) probability flux $[57,62]$. They are distinguished by means of a time parameter $t_{c}$, such that the wave packet passes through $x_{1}$ completely before $t_{\mathrm{c}}$, and after some finite duration with zero flux the reflected part returns through $x_{1}$ with a negative current and the transmitted part passes $x_{2}$. The expressions read

$$
\begin{aligned}
& \tau_{\mathrm{T}}=\langle t\rangle_{x_{2}}^{\text {out }}-\langle t\rangle_{x_{1}}^{\text {in }}, \\
& \tau_{\mathrm{R}}=\langle t\rangle_{x_{1}}^{\text {out }}-\langle t\rangle_{x_{1}}^{\text {in }},
\end{aligned}
$$

where

$$
\begin{aligned}
\langle t\rangle_{x_{2}}^{\text {out }} & =\frac{\int_{-\infty}^{\infty} \mathrm{d} t t J\left(t, x_{2}\right)}{\int_{-\infty}^{\infty} \mathrm{d} t J\left(t, x_{2}\right)}, \\
\langle t\rangle_{x_{1}}^{\text {in }} & =\int_{-\infty}^{t_{\mathrm{c}}} \mathrm{d} t t J\left(t, x_{1}\right), \\
\langle t\rangle_{x_{1}}^{\text {out }} & =-\frac{\int_{t_{\mathrm{c}}}^{\infty} \mathrm{d} t t J\left(t, x_{1}\right)}{\int_{t_{\mathrm{c}}}^{\infty} \mathrm{d} t\left|J\left(t, x_{1}\right)\right|} .
\end{aligned}
$$


A discussion of the consequences of this definition can be found in Ref. [63]. Transmission and reflection times are often supposed to be related to the dwell time $\tau_{\mathrm{D}}$, i.e. to the time a particle spends in a given region (see Section 2.2.3), by the relation [62]

$$
\tau_{\mathrm{D}}(k)=|T(k)|^{2} \tau_{T}+|R(k)|^{2} \tau_{R},
$$

where $T(k)$ and $R(k)$ are the transmission and reflection amplitudes of the planewave solutions for the given potential, respectively. A systematic approach to define and classify quantum transmission and reflection times has been given by Brouard et al. [64].

\subsection{Dwell times}

In contrast to arrival times considered in the previous sections, the dwell or sojourn time is the mean time spent by a particle in a given region $x_{1} \leq x \leq x_{2}$. Although for free classical particles the relation between arrival times and dwell times is very close, the quantum case is different and gave reason to much discussion. In particular, the question of traversal or reflection times in the presence of a potential barrier has been extensively studied and controversially discussed.

In Sections 2.2.1 and 2.2.2, classical dwell times are considered and a connection to flux-flux correlation functions is pointed out. The quantum case is reviewed in Section 2.2.3 and a particular dwell-time distribution which is based on a widely accepted dwell-time operator is investigated in detail.

For convenience, the main focus in this work is placed on free dwell times, since the problem of an operational understanding of quantum dwell-time distributions is completely unclear so far and it will be investigated in Chapter 4 for the case of no external interaction.

\subsubsection{Classical dwell time}

In the following, classical dwell times in the interval $x_{1} \leq x \leq x_{2}$ for one-dimensional motion are considered. The interval width is denoted by

$$
L=x_{2}-x_{1}>0 .
$$

For one classical particle, the dwell time is given in general by the expression [62]

$$
t_{\mathrm{D}}=\int_{-\infty}^{\infty} \mathrm{d} t \chi_{\left[x_{1}, x_{2}\right]}(x(t))
$$

where $\chi_{\left[x_{1}, x_{2}\right]}(x)$ is the characteristic function of the interval $\left[x_{1}, x_{2}\right]$ and $x(t)$ is the spatial phase-space coordinate. For free motion one has $p(t)=p_{0}$ and $x(t)=$ $x_{0}+p_{0} t / m$, and Eq.(2.47) simplifies to

$$
t_{\mathrm{D}}=\frac{m L}{\left|p_{0}\right|}
$$


which is independent of the initial position $x_{0}$. For a given phase-space distribution function $\varrho_{t=0}\left(x_{0}, p_{0}\right)$, the ensemble average of Eq. (2.47) is

$$
\begin{aligned}
\left\langle t_{\mathrm{D}}\right\rangle & =\int_{-\infty}^{\infty} \mathrm{d} x_{0} \int_{-\infty}^{\infty} \mathrm{d} p_{0} \varrho_{0}\left(x_{0}, p_{0}\right) \int_{-\infty}^{\infty} \mathrm{d} t \chi_{\left[x_{1}, x_{2}\right]}(x(t)) \\
& =\int_{-\infty}^{\infty} \mathrm{d} t \int_{-\infty}^{\infty} \mathrm{d} x \int_{-\infty}^{\infty} \mathrm{d} p \varrho_{t}(x, p) \chi_{\left[x_{1}, x_{2}\right]}(x) \\
& =\int_{-\infty}^{\infty} \mathrm{d} \tau \int_{x_{1}}^{x_{2}} \mathrm{~d} x \varrho_{t}(x),
\end{aligned}
$$

where in Eq. (2.50) Liouville's theorem has been used and $\varrho_{t}(x)=\int \mathrm{d} p \varrho_{t}(x, p)$ is the local probability density for the position $x$.

\subsubsection{Classical flux-flux correlation function}

It is widely known that $t_{\mathrm{D}}$ can be written as the first moment of a flux-flux correlation function, although this connection is often formulated within a micro-canonical picture [65]. A different approach for the free case is given in the following, which leads to a physically intuitive expression and which provides an interesting result when quantized, as shown in Section 4.2. Starting from Eq. (2.48) one has

$$
\begin{aligned}
t_{\mathrm{D}} & =\frac{m\left(x_{2}-x_{1}\right)}{\left|p_{0}\right|} \\
& =\int_{0}^{\infty} \mathrm{d} \tau \tau\left\{\delta\left(\tau-\frac{m\left(x_{2}-x_{1}\right)}{p_{0}}\right)+\delta\left(\tau-\frac{m\left(x_{2}-x_{1}\right)}{-p_{0}}\right)\right\} \\
& =\int_{0}^{\infty} \mathrm{d} \tau \tau\left\{\delta\left(\tau-\frac{m\left(x_{2}-x_{1}\right)}{p_{0}}\right)+\delta\left(\tau-\frac{m\left(x_{1}-x_{2}\right)}{p_{0}}\right)\right. \\
& \left.-\delta\left(\tau-\frac{m\left(x_{1}-x_{1}\right)}{p_{0}}\right)-\delta\left(\tau-\frac{m\left(x_{2}-x_{2}\right)}{p_{0}}\right)\right\} \\
= & \sum_{i, j=1}^{2}(-1)^{i+j+1} \int_{0}^{\infty} \mathrm{d} \tau \tau \delta\left(\tau-\frac{m\left(x_{i}-x_{j}\right)}{p_{0}}\right)
\end{aligned}
$$

where the self-correlation terms are zero and have been introduced for later purpose. The expression can be further manipulated to obtain

$$
\begin{gathered}
t_{\mathrm{D}}=\sum_{i, j=1}^{2}(-1)^{i+j+1} \int_{0}^{\infty} \mathrm{d} \tau \tau \frac{\left|p_{0}\right|}{m} \delta\left[x_{0}+\frac{p_{0}}{m}\left(\tau+\frac{m}{p_{0}}\left(x_{i}-x_{0}\right)\right)-x_{j}\right] \\
=\sum_{i, j=1}^{2}(-1)^{i+j+1} \int_{0}^{\infty} \mathrm{d} \tau \tau \int_{-\infty}^{\infty} \mathrm{d} t \delta\left(t-\frac{m}{p_{0}}\left(x_{i}-x_{0}\right)\right) \frac{\left|p_{0}\right|}{m} \delta\left(x_{0}+\frac{p_{0}}{m}(t+\tau)-x_{j}\right) \\
=\sum_{i, j=1}^{2}(-1)^{i+j+1} \int_{0}^{\infty} \mathrm{d} \tau \tau \int_{-\infty}^{\infty} \mathrm{d} t \frac{\left|p_{0}\right|}{m} \delta\left(x_{0}+\frac{p_{0}}{m} t-x_{i}\right) \\
\times \frac{\left|p_{0}\right|}{m} \delta\left(x_{0}+\frac{p_{0}}{m}(t+\tau)-x_{j}\right) .
\end{gathered}
$$


Since $p=p_{0}$ and $x(t)=x_{0}+p_{0} t / m$ for free motion, Eq. (2.53) can be written, using Eq. (2.7), in terms of the classical phase-space variable $\mathcal{J}_{\text {tot }}^{\text {cl }}$ corresponding to the sum of the positive flux and minus the negative flux,

$$
\begin{array}{r}
t_{\mathrm{D}}=\int_{0}^{\infty} \mathrm{d} \tau \tau \int_{-\infty}^{\infty} \mathrm{d} t\left(\mathcal{J}_{\mathrm{tot}}^{\mathrm{cl}}\left(x(t), p ; x_{1}\right) \mathcal{J}_{\mathrm{tot}}^{\mathrm{cl}}\left(x(t+\tau), p ; x_{2}\right)\right. \\
+\mathcal{J}_{\mathrm{tot}}^{\mathrm{cl}}\left(x(t), p ; x_{2}\right) \mathcal{J}_{\mathrm{tot}}^{\mathrm{cl}}\left(x(t+\tau), p ; x_{1}\right)-\mathcal{J}_{\mathrm{tot}}^{\mathrm{cl}}\left(x(t), p ; x_{1}\right) \mathcal{J}_{\mathrm{tot}}^{\mathrm{cl}}\left(x(t+\tau), p ; x_{1}\right) \\
\left.-\mathcal{J}_{\text {tot }}^{\mathrm{cl}}\left(x(t), p ; x_{2}\right) \mathcal{J}_{\text {tot }}^{\mathrm{cl}}\left(x(t+\tau), p ; x_{2}\right)\right) .
\end{array}
$$

This is an intuitive result: Eq. (2.54) counts all correlations between arrivals at $x_{1}$ and at $x_{2}$ separated by a time $\tau$, irrespective of their occurrence (thus the integration over $t$ ), and takes the first moment of this correlation function. The usefulness of Eq. (2.54) is twofold: Firstly, it can be easily generalized to the case of an external potential, secondly it can be easily quantized, since the quantum mechanical flux operator is known. This will be shown in Section 4.2. Moreover, its form as a flux-flux correlation function prepares the ground for a possible operational understanding of dwell-time distributions in quantum mechanics.

\subsubsection{Quantum dwell times}

The first definition of a quantum dwell time $\tau_{\mathrm{D}}$ for the stationary regime has been proposed by Smith [66]. Its one-dimensional form reads [67]

$$
\tau_{\mathrm{D}}(k)=\frac{1}{j(k)} \int_{x_{1}}^{x_{2}} \mathrm{~d} x\left|\phi_{k}(x)\right|^{2},
$$

where $j(k)$ is the incoming flux associated with the stationary state $\phi_{k}(x)$. For the free case, $\phi_{k}(x)$ are plane wave solutions of the stationary Schrödinger equation, $\phi_{k}(x)=\mathrm{e}^{\mathrm{i} k x} / \sqrt{2 \pi}$ and $j(k)=\hbar k /(2 \pi m)$, which leads to $\tau_{\mathrm{D}}(k)=m L /(\hbar k)$. This corresponds to the classical result (2.48).

Several authors proposed operational-based approaches to stationary dwell times by means of a "Larmor clock" [68, 69, 67]. The idea is to use a homogeneous magnetic field which covers the region $\left[x_{1}, x_{2}\right]$ and to measure the amount of spin rotations of incident spin- $\frac{1}{2}$ particles. Several relations of this concept to the Smith dwell time are pointed out in Refs. [67, 13]. A related proposal has been put forward by Büttiker and Landauer [61] in terms of a time-modulated barrier which interchanges phonon quanta with the wave function.

Moreover, in the framework of the Feynman path integral formalism Sokolovski and Baskin [70] obtained an expression for quantum traversal times that is complexvalued, where the real and the imaginary part can be related to two different versions of the Larmor time, respectively. In particular, the real part is the phase-time delay associated with Eq. (2.39). Complex-valued traversal times have also been investigated in Refs. [65, 71, 72]. A measurement-based procedure that yields both the real and the imaginary part of the traversal time by means of optical tunneling has been proposed in Ref. [55]. 
A time-dependent formulation of dwell times can be given by the instantaneous probability that the particle is inside the barrier [73, 62],

$$
\tau_{\mathrm{D}}=\int_{-\infty}^{\infty} \mathrm{d} t \int_{x_{1}}^{x_{2}} \mathrm{~d} x|\psi(x, t)|^{2},
$$

which is the quantum version of the classical result (2.51). In the limit of monochromatic wave packets, the two formulas (2.55) and (2.56) yield the same result.

In the following section, a quantization of the classical expression for a dwell time is considered and the corresponding dwell-time distribution for free motion is studied.

\subsubsection{Quantum dwell-time operator}

For a quantum dwell-time operator, Ekstein and Siegert [26] proposed a quantization of the classical expression (2.47) which reads

$$
\widehat{T}_{\mathrm{D}}=\int_{-\infty}^{\infty} \mathrm{d} \tau e^{\mathrm{i} H \tau / \hbar} \chi_{\left[x_{1}, x_{2}\right]}(\widehat{x}) e^{-\mathrm{i} H \tau / \hbar} .
$$

The expectation value of this operator equals the dwell time $\tau_{\mathrm{D}}$ of Eq. (2.56),

$$
\tau_{\mathrm{D}}=\left\langle\psi(t=0)\left|\widehat{T}_{\mathrm{D}}\right| \psi(t=0)\right\rangle .
$$

The operator $\widehat{T}_{\mathrm{D}}$ has been extensively studied in the literature $[74,75,76]$ and it is widely accepted as a dwell-time operator. For the sake of convenience, only free dwell times are considered, i.e. the Hamiltonian is given in the following by $H=\widehat{p}^{2} / 2 m$. It can be shown that $\widehat{T}_{\mathrm{D}}$ is essentially self-adjoint and commutes with $H$ [76]. Consequently, the eigenstates $\left|t_{k}^{ \pm}\right\rangle$of $\widehat{T}_{\mathrm{D}}$ are superpositions of momentum eigenstates and they are given by

$$
\left|t_{k}^{ \pm}\right\rangle=\frac{1}{\sqrt{2}}\left(|k\rangle \pm e^{\mathrm{i} k\left(x_{1}+x_{2}\right)}|-k\rangle\right), \quad k>0 .
$$

They belong to the following two different eigenvalues in each energy eigenspace:

$$
t_{k}^{ \pm}=\frac{m L}{\hbar k}\left(1 \pm \frac{\sin (k L)}{k L}\right), \quad k>0 .
$$

For $k \rightarrow 0, t_{k}^{+}$diverges whereas $t_{k}^{-}$goes to zero. The stationary dwell time $\tau_{\mathrm{D}}(k)$ of Eq. (2.55) is recovered by the average of $t_{k}^{+}$and $t_{k}^{-}$,

$$
t_{\mathrm{D}}(k)=\frac{t_{k}^{+}+t_{k}^{-}}{2}=\frac{m L}{\hbar k} .
$$

An example for the form of the eigenvalues $t_{k}^{ \pm}$is shown in Fig. 2.1. It is important to note that the eigenstates of $\widehat{T}_{\mathrm{D}}$ are complete, i.e.

$$
\widehat{1}=\int_{0}^{\infty} \mathrm{d} k\left(\left|t_{k}^{+}\right\rangle\left\langle t_{k}^{+}|+| t_{k}^{-}\right\rangle\left\langle t_{k}^{-}\right|\right) .
$$

This completeness relation becomes essential in the following section, where the distribution of dwell times is derived. 


\subsubsection{Dwell-time distribution for free motion}

The distribution of dwell times for the operator $\widehat{T}_{\mathrm{D}}$ and for a state $|\psi\rangle$ is given by

$$
\Pi_{\mathrm{D}}(t)=\left\langle\psi\left|\delta\left(\widehat{T}_{\mathrm{D}}-t\right)\right| \psi\right\rangle .
$$

It exhibits some interesting features which do not seem to be extensively studied in the literature. This is shown in the following.

Inserting the completeness relation (2.62) yields

$$
\Pi_{\mathrm{D}}(t)=\int_{0}^{\infty} \mathrm{d} k\left(\left|\left\langle t_{k}^{+} \mid \psi\right\rangle\right|^{2} \delta\left(t_{k}^{+}-t\right)+\left|\left\langle t_{k}^{-} \mid \psi\right\rangle\right|^{2} \delta\left(t_{k}^{-}-t\right)\right) .
$$

To evaluate the integral, the zeros of the functions

$$
F_{ \pm}(k)=\frac{m L}{\hbar k}\left(1 \pm \frac{\sin (k L)}{k L}\right)-t
$$

have to be determined. Since $F^{ \pm}(k)=0$ is a transcendental equation, it cannot be solved analytically but its zeros can be determined numerically. In the following they are denoted by $k_{\alpha}^{ \pm}(t)$. This yields for the dwell-time distribution

$$
\begin{gathered}
\Pi_{\mathrm{D}}(t)=\int_{0}^{\infty} \mathrm{d} k \sum_{\alpha}\left(\left|\left\langle t_{k}^{+} \mid \psi\right\rangle\right|^{2} \frac{\delta\left(k-k_{\alpha}^{+}(t)\right)}{\left|F_{+}^{\prime}\left(k_{\alpha}^{+}(t)\right)\right|}+\left|\left\langle t_{k}^{-} \mid \psi\right\rangle\right|^{2} \frac{\delta\left(k-k_{\alpha}^{-}(t)\right)}{\left|F_{-}^{\prime}\left(k_{\alpha}^{-}(t)\right)\right|}\right) \\
=\frac{1}{2} \sum_{\alpha}\left(\frac{\left|\widetilde{\psi}\left(k_{\alpha}^{+}(t)\right)+\mathrm{e}^{-\mathrm{i} k_{\alpha}^{+}(t)\left(x_{1}+x_{2}\right)} \widetilde{\psi}\left(-k_{\alpha}^{+}(t)\right)\right|^{2}}{\left|F_{+}^{\prime}\left(k_{\alpha}^{+}(t)\right)\right|}\right. \\
\left.+\frac{\left|\widetilde{\psi}\left(k_{\alpha}^{-}(t)\right)-\mathrm{e}^{-\mathrm{i} k_{\alpha}^{-}(t)\left(x_{1}+x_{2}\right)} \widetilde{\psi}\left(-k_{\alpha}^{-}(t)\right)\right|^{2}}{\left|F_{-}^{\prime}\left(k_{\alpha}^{-}(t)\right)\right|}\right) .
\end{gathered}
$$

To give an example for this expression, the distribution $\Pi_{D}(t)$ is numerically evaluated for the three wave packets shown in Fig. 2.1.

It is obvious that $\Pi_{\mathrm{D}}(t)$ exhibits some resonance features. This is related to the fact that in Eq. (2.67) the value of the first derivative at the zeros enters in the denominator. At the points for which the values of $t_{k}^{+}$and $t_{k}^{-}$agree, i.e. at $k_{0}=n \pi / L$, $n=1,2,3, \ldots$, either $F_{+}^{\prime}$ or $F_{-}^{\prime}$ are zero, since $F_{ \pm}^{\prime}(k)=-\frac{m L}{\hbar k^{2}} \pm \frac{m L}{\hbar k^{2}} \cos (k L) \mp$ $\frac{2 m}{\hbar k^{3}} \sin (k L)$. Thus, at these points the value of $\Pi_{\mathrm{D}}(t)$ diverges which is clearly visible in Fig. 2.1. For the case (a), the wave packet is located in a region in $k$-space, where $t_{k}^{+}$and $t_{k}^{-}$have distinct values and the distribution has two peaks centered around these two values. A similar feature has been described in Ref. [76], where a bimodal dwell-time distribution for particles with small energies is predicted. In the case (b) of Fig. 2.1, the momentum distribution is located around some crossing point $k_{0}$, and consequently the dwell-time distribution exhibits a very sharp peak at the corresponding value. This becomes more conspicuous in case (c), where the wave packet spreads over some of these crossing points, and each of them accounts for a sharp peak in the distribution. A similar resonance structure for the free dwell-time distribution has been indicated very recently by Alonso et al. [77], but it has not been further investigated or commented within this publication. 

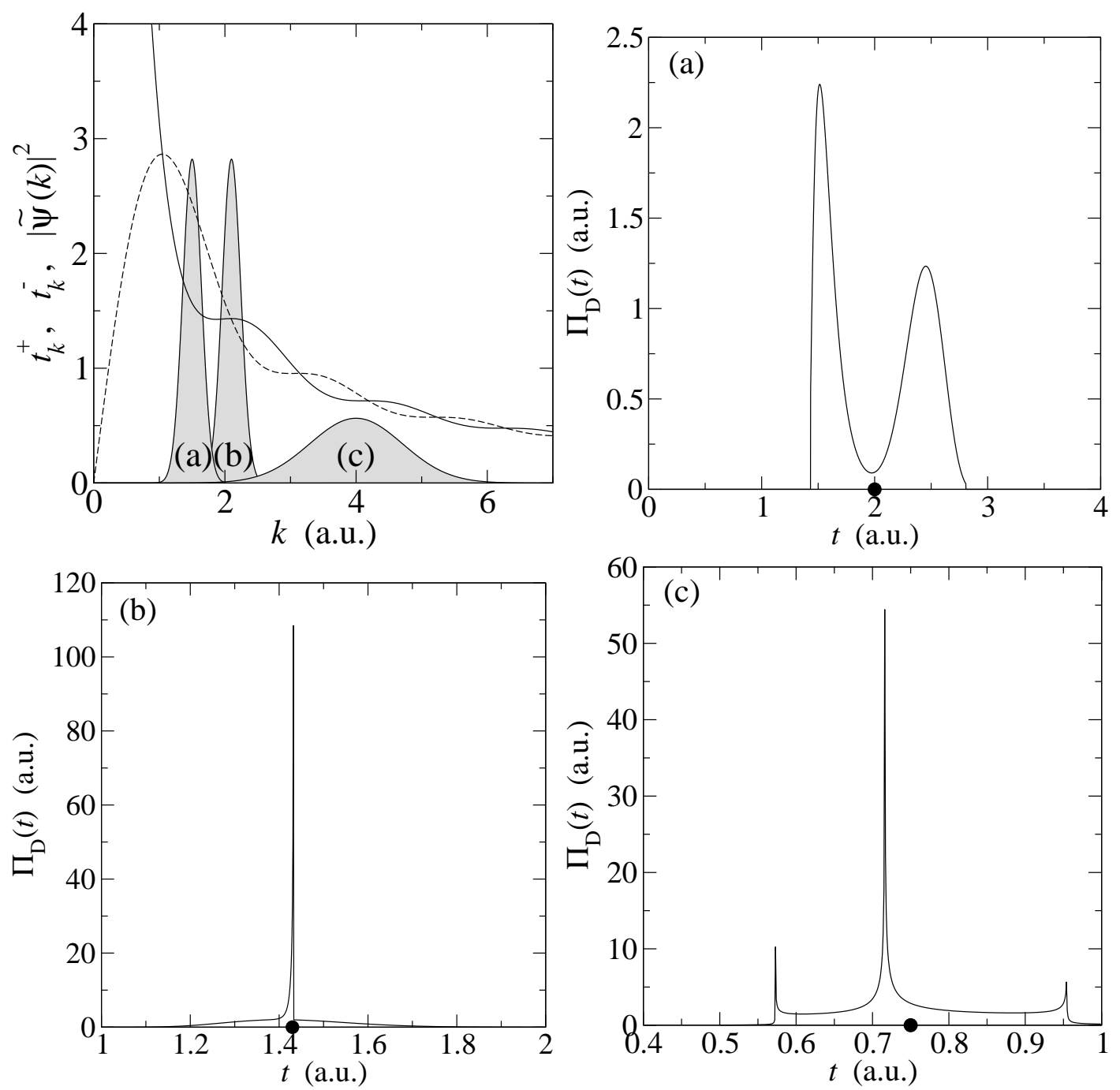

Figure 2.1: Dwell time eigenvalues $t_{k}^{+}$(solid line) and $t_{k}^{-}$(dashed line) and ideal dwell-time distributions $\Pi_{\mathrm{D}}(t)$ with Eq. (2.67) for three different minimumuncertainty wave packets $|\widetilde{\psi}(k)|^{2}$. The interval length is $L=3$. Shown is $\Pi_{\mathrm{D}}(t)$ for: (a) $\langle k\rangle=1.5, \Delta k=0.1$; (b) $\langle k\rangle=2.1, \Delta k=0.1$; (c) $\langle k\rangle=4.0, \Delta k=0.5$. All numbers are in atomic units, see Appendix D. For comparison, the mean classical dwell time $t_{\mathrm{D}}=L / v$ is indicated by the circle.

\subsubsection{Moments of the dwell-time distribution}

To derive the moments of the dwell-time distribution $\Pi_{\mathrm{D}}(t)$, it is advantageous to rewrite Eq. (2.63) in the following manner:

$$
\Pi_{\mathrm{D}}(t)=\frac{1}{2 \pi}\left\langle\psi\left|\int_{-\infty}^{\infty} \mathrm{d} \lambda \mathrm{e}^{\mathrm{i} \lambda\left(\widehat{T}_{\mathrm{D}}-t\right)}\right| \psi\right\rangle
$$


Using the completeness relation (2.62) one has $\mathrm{e}^{\mathrm{i} \lambda \widehat{T}_{\mathrm{D}}}=\int_{0}^{\infty} \mathrm{d} k \sum_{\alpha= \pm}\left|t_{k}^{\alpha}\right\rangle\left\langle t_{k}^{\alpha}\right| \mathrm{e}^{\mathrm{i} \lambda t_{k}^{\alpha}}$ and thus it follows with Eq. (2.59)

$$
\begin{aligned}
\Pi_{\mathrm{D}}(t)=\frac{1}{4 \pi} \int_{-\infty}^{\infty} \mathrm{d} \lambda \mathrm{e}^{-\mathrm{i} \lambda t} \int_{0}^{\infty} \mathrm{d} k\left[\left(\mathrm{e}^{\mathrm{i} \lambda t_{k}^{+}}+\mathrm{e}^{\mathrm{i} \lambda t_{k}^{-}}\right)\left(|\widetilde{\psi}(k)|^{2}+|\widetilde{\psi}(-k)|^{2}\right)\right. \\
\left.+\left(\mathrm{e}^{\mathrm{i} \lambda t_{k}^{+}}-\mathrm{e}^{\mathrm{i} \lambda t_{k}^{-}}\right) 2 \operatorname{Re}\left\{\mathrm{e}^{-\mathrm{i} k\left(x_{1}+x_{2}\right)} \overline{\widetilde{\psi}(k)} \widetilde{\psi}(-k)\right\}\right] .
\end{aligned}
$$

The first moment of this distribution is

$$
\begin{aligned}
&\left\langle\widehat{T}_{\mathrm{D}}\right\rangle= \int_{-\infty}^{\infty} \mathrm{d} t t \Pi_{\mathrm{D}}(t) \\
&=\frac{\mathrm{i}}{2} \int_{-\infty}^{\infty} \mathrm{d} \lambda \delta^{\prime}(\lambda) \int_{0}^{\infty} \mathrm{d} k\left[\left(\mathrm{e}^{\mathrm{i} \lambda t_{k}^{+}}+\mathrm{e}^{\mathrm{i} \lambda t_{k}^{-}}\right)\left(|\widetilde{\psi}(k)|^{2}+|\widetilde{\psi}(-k)|^{2}\right)\right. \\
&\left.\quad+\left(\mathrm{e}^{\mathrm{i} \lambda t_{k}^{+}}-\mathrm{e}^{\mathrm{i} \lambda t_{k}^{-}}\right) 2 \operatorname{Re}\left\{\mathrm{e}^{-\mathrm{i} k\left(x_{1}+x_{2}\right)} \overline{\widetilde{\psi}(k)} \widetilde{\psi}(-k)\right\}\right] \\
&=-\frac{\mathrm{i}}{2} \int_{0}^{\infty} \mathrm{d} k\left[\mathrm{i}\left(t_{k}^{+}+t_{k}^{-}\right)\left(|\widetilde{\psi}(k)|^{2}+|\widetilde{\psi}(-k)|^{2}\right)\right. \\
&\left.+\mathrm{i}\left(t_{k}^{+}-t_{k}^{-}\right) 2 \operatorname{Re}\left\{\mathrm{e}^{-\mathrm{i} k\left(x_{1}+x_{2}\right)} \overline{\widetilde{\psi}(k)} \widetilde{\psi}(-k)\right\}\right] .
\end{aligned}
$$

With Eq. (2.60) and by extending the integral over the whole axis it follows that

$$
\left\langle\widehat{T}_{\mathrm{D}}\right\rangle=\int_{-\infty}^{\infty} \mathrm{d} k \frac{m L}{\hbar|k|}\left(|\widetilde{\psi}(k)|^{2}+\overline{\widetilde{\psi}(k)} \widetilde{\psi}(-k) \frac{\sin (k L) \mathrm{e}^{-\mathrm{i} k\left(x_{1}+x_{2}\right)}}{k L}\right) .
$$

The second term, an interference term, is absent for particles with only positive momentum support and the result can then be understood as a quantum average over the classical dwell time $m L / \hbar k$. An operational understanding of this average dwell time for left incoming particles has been given by Golub et al. [78], which is reviewed in Section 4.1.

For the second moment as well as for higher moments the same procedure as above applies. One has with two partial integrations

$$
\begin{aligned}
\left\langle\widehat{T}_{\mathrm{D}}^{2}\right\rangle= & \int_{-\infty}^{\infty} \mathrm{d} t t^{2} \Pi_{\mathrm{D}}(t) \\
= & -\frac{1}{2} \int_{0}^{\infty} \mathrm{d} k\left[-\left(t_{k}^{+2}+t_{k}^{-2}\right)\left(|\widetilde{\psi}(k)|^{2}+|\widetilde{\psi}(-k)|^{2}\right)\right. \\
& \left.\quad-\left(t_{k}^{+2}-t_{k}^{-2}\right) 2 \operatorname{Re}\left\{\mathrm{e}^{-\mathrm{i} k\left(x_{1}+x_{2}\right)} \widetilde{\widetilde{\psi}(k)} \widetilde{\psi}(-k)\right\}\right] \\
= & \int_{-\infty}^{\infty} \mathrm{d} k \frac{m^{2} L^{2}}{\hbar^{2}|k|^{2}}\left[|\widetilde{\psi}(k)|^{2}\left(1+\frac{\sin ^{2}(k L)}{k^{2} L^{2}}\right)+\widetilde{\widetilde{\psi}(k)} \widetilde{\psi}(-k) \frac{2 \sin (k L) \mathrm{e}^{-\mathrm{i} k\left(x_{1}+x_{2}\right)}}{k L}\right] .
\end{aligned}
$$

No operational understanding has been found so far for $\left\langle\widehat{T}_{\mathrm{D}}^{2}\right\rangle$ and higher moments, as well as for the dwell-time distribution $\Pi_{\mathrm{D}}(t)$. This means that it is a priori 
not clear, whether some experiment could measure such distributions as shown in Fig. 2.1, even for the free case.

In Chapter 4, a new relation between dwell-time distributions and quantum fluxflux correlation functions is pointed out. This may help to improve the operational understanding of dwell-time distributions in terms of those correlation functions.

\subsubsection{An alternative proposal for free dwell times}

For free dwell times, one may argue to consider the classical expression (2.48) instead of Eq. (2.47) for quantization. This yields an operator for free arrival times which reads

$$
\widehat{t}_{\mathrm{D}}=\frac{m L}{|\widehat{p}|} .
$$

The eigenfunctions of this operator are momentum eigenfunctions, $| \pm k\rangle, k>0$, and

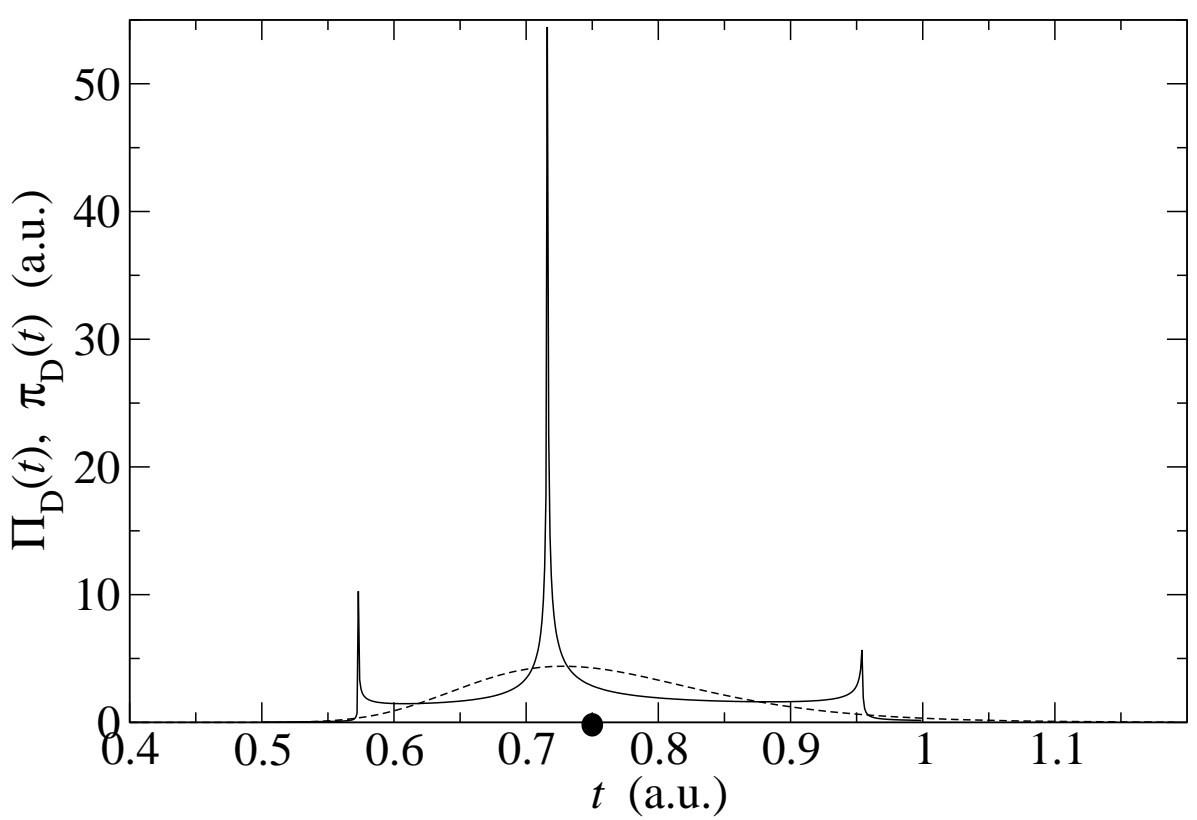

Figure 2.2: Comparison of the dwell-time distribution $\Pi_{\mathrm{D}}(t)$ (solid line), corresponding to the operator (2.57) and the distribution $\pi_{\mathrm{D}}(t)$ (dashed line), corresponding to the operator (2.77). In contrast to $\Pi_{\mathrm{D}}(t), \pi_{\mathrm{D}}(t)$ exhibits no resonance structure. The initial wave packet is the same as for Fig. 2.1c. For comparison, the mean classical dwell time $t_{\mathrm{D}}=L / v$ is indicated by the circle.

the corresponding eigenvalues $m L / \hbar|k|$ are twofold degenerated. The distribution of dwell times for this operator is given by

$$
\begin{aligned}
\pi_{\mathrm{D}}(t) & =\left\langle\psi\left|\delta\left(\widehat{t}_{\mathrm{D}}-t\right)\right| \psi\right\rangle \\
& =\int_{-\infty}^{\infty} \mathrm{d} k\left\langle\psi\left|\delta\left(\frac{m L}{\hbar|k|}-t\right)\right| k\right\rangle\langle k \mid \psi\rangle \\
& =\frac{m L}{\hbar t^{2}}\left[\left|\widetilde{\psi}\left(\frac{m L}{\hbar t}\right)\right|^{2}+\left|\widetilde{\psi}\left(-\frac{m L}{\hbar t}\right)\right|^{2}\right] .
\end{aligned}
$$


It is clearly recognizable that $\pi_{\mathrm{D}}(t)$ does not exhibit resonances as it was the case for $\Pi_{D}(t)$. This is illustrated in Fig. 2.2, where $\Pi_{D}$ and $\pi_{D}(t)$ are compared for the same example that has been shown in Fig. 2.1c. It can be seen that $\pi_{\mathrm{D}}(t)$ provides a "smoothed" version of $\Pi_{D}(t)$ and, moreover, that the asymmetry of $\Pi_{D}(t)$ is present in the new dwell time distribution, too. As for different versions for arrival-time distributions, an operational approach to dwell times is needed to show which one of both distributions is a measurable quantity. A first proposal for such an operational approach is given in Chapter 4 . 


\section{Chapter 3}

\section{Operational approaches to quantum arrival times}

Parallel to the efforts of defining ideal quantum arrival times or arrival-time distributions, a recent interest in operational approaches to these quantities has arised $[22,24]$. In this context, "operational" means "measurement-based". The aim of this approach is to model a physical situation including measurement devices, detectors, etc., in a way that there is a close connection between the theoretical model and a realistic experiment. These operational models can provide new insights into the field and, moreover, they may encourage experiments for an investigation of arrivaltime distributions and related quantum effects. The importance of this is due to the fact that the classical picture for measuring the time-of-flight is present almost everywhere in experimental textbooks (cf., e.g. Ref. [2]). In contrast, the recent progress in laser technology and the ability to manipulate atomic motion and the internal states of quantum systems nowadays allows the experimental investigation of specific issues concerning time in quantum mechanics.

Early contributions to an operational understanding of arrival times are due to Allcock [6] who considered the absorption rate of particles in an absorbing potential that has the meaning of a "detector". Further progress in this direction has been made by Muga et al. [7] by deriving properly constructed absorbing potentials and, more basically, by Werner [34]. Blanchard and Jadczyk developed their event- enhanced quantum theory that is based on the use of complex potentials in the time evolution to explain the occurrence of events and their timing $[79,80]$. In contrast to a phenomenological introduction of absorbing potentials into the Schrödinger equation, Halliwell presented a more realistic two-channel detector model [22] in which the initially excited detector decays due to the presence of the particle. Several "toy models" for arrival-time measurements have been put forward by Aharonov et al. [8].

A recent quantum optical model to arrival-time distribution has been proposed by Muga et al. [23] and extensively investigated in Ref. [24]. The central idea of this model is to illuminate the region $x \geq 0$ by a laser and to measure the temporal distribution of the first photon emitted spontaneously from a two-level wave packet that impinges on the laser field from the left. This first-photon distribution is 
considered as an operational approximation to the arrival-time distribution of the atom at $x_{\mathrm{A}}=0$ and can be investigated in different ideal limits. Many consequences of this approach have been studied recently in a series of papers [81, 82, 83, 84].

The central topic in this work is the further development and investigation of the quantum optical model of Ref. [24]. An introduction to the theory of photon emissions which is based on the quantum jump approach is given in Section 3.1. In Section 3.2 the basic arrival-time model is described and new consequences are investigated in the following sections.

\subsection{The quantum jump approach}

In the last twenty years, different methods have been developed for the description of single fluorescing quantum systems. In the present work, the quantum jump approach of Hegerfeldt and Wilser [85, 86, 87] will be used. It is equivalent to the Monte-Carlo wave-function approach [88] and to the method of quantum trajectories [89]. A detailed review can be found by Plenio and Knight [90]. In this section the basic idea of the quantum jump approach is explained and the approach is used to describe the time evolution of a single two-level atom with quantized center-of-mass $(\mathrm{cm})$ motion in the presence of spatially confined laser fields. This will provide the technical basis for the quantum optical arrival-time model of this work.

\subsubsection{Conditional time evolution}

The observation of photons that are emitted from a quantum system corresponds to a continuous measurement. To circumvent the consequential problems, it is suggestive to employ discrete gedanken measurements being performed by an ideal detector at times $t_{i}$ which have a separation of $\Delta t$. Some conditions on $\Delta t$ are required here: It has to be small compared with the mean lifetime of the excited state but large enough to avoid the quantum Zeno effect, i.e. the freezing of the atom's time evolution. For optical transitions, these conditions lead to a value of

$$
\Delta t \approx 10^{-12} \mathrm{~s} .
$$

In the following one considers a system, consisting of a laser-driven quantum particle with internal states $|n\rangle$ and the quantized radiation field. The laser field is treated classically. The state of the system at time $t=0$ is given by $\left|0_{\mathrm{ph}}\right\rangle\left|\Psi_{0}\right\rangle$, where $\left\langle x \mid \Psi_{0}\right\rangle=\sum_{n} \psi_{0}^{(n)}(x)|n\rangle$ is a multi-component wave function describing the internal state of the atom and $\left|0_{\mathrm{ph}}\right\rangle$ is the vacuum state of the field. Let $H$ be the complete Hamilton operator in the Schrödinger picture, consisting of the free part of the atom and the field, $H_{0}$, and an interaction part, $H_{1}$, which includes the laser driving and the dipole interaction of the atom with the field. The corresponding timedevelopment operator is denoted by $U\left(t, t_{0}\right)$.

If no photon is observed, the measurement corresponds to a projection onto the no-photon subspace with the projector $\widehat{P}_{0}=\left|0_{\mathrm{ph}}\right\rangle \widehat{1}_{\mathrm{A}}\left\langle 0_{\mathrm{ph}}\right|$, where $\widehat{1}_{\mathrm{A}}$ denotes the 
unity operator with respect to the atomic states [91]. The state after $n$ measurements without photon detection reads

$$
\begin{aligned}
\left|0_{\mathrm{ph}}\right\rangle\left|\boldsymbol{\Psi}\left(t_{n}\right)\right\rangle & =\widehat{P}_{0} U\left(t_{n}, t_{n-1}\right) \widehat{P}_{0} \cdots \widehat{P}_{0} U\left(t_{1}, t_{0}\right)\left|0_{\mathrm{ph}}\right\rangle\left|\Psi_{0}\right\rangle \\
& =\left|0_{\mathrm{ph}}\right\rangle U_{\mathrm{c}}\left(t_{n}, t_{0}\right)\left|\boldsymbol{\Psi}_{0}\right\rangle
\end{aligned}
$$

where

$$
U_{\mathrm{c}}\left(t_{n}, t_{0}\right)=\left\langle 0_{\mathrm{ph}}\left|U\left(t_{n}, t_{n-1}\right)\right| 0_{\mathrm{ph}}\right\rangle\left\langle 0_{\mathrm{ph}}|\cdots| 0_{\mathrm{ph}}\right\rangle\left\langle 0_{\mathrm{ph}}\left|U\left(t_{1}, t_{0}\right)\right| 0_{\mathrm{ph}}\right\rangle
$$

is the atomic time-development operator under the condition that no photon has been detected. It is derived in the interaction picture with respect to $H_{1}$, given by

$$
\begin{aligned}
U_{\mathrm{c}}^{\mathrm{I}}\left(t_{i}, t_{i-1}\right) & =\mathrm{e}^{\mathrm{i} H_{0} t_{i} / \hbar}\left\langle 0_{\mathrm{ph}}\left|U\left(t_{i}, t_{i-1}\right)\right| 0_{\mathrm{ph}}\right\rangle \mathrm{e}^{-\mathrm{i} H_{0} t_{i} / \hbar} \\
& =\left\langle 0_{\mathrm{ph}}\left|U^{\mathrm{I}}\left(t_{i}, t_{i-1}\right)\right| 0_{\mathrm{ph}}\right\rangle,
\end{aligned}
$$

where $U^{\mathrm{I}}\left(t_{i}, t_{i-1}\right)$ can be expressed by the Dyson series, which yields

$$
\begin{aligned}
U_{\mathrm{c}}^{\mathrm{I}}\left(t_{i}, t_{i-1}\right)=\widehat{1}_{\mathrm{A}}-\frac{\mathrm{i}}{\hbar} & \int_{t_{i-1}}^{t_{i}} \mathrm{~d} t^{\prime}\left\langle 0_{\mathrm{ph}}\left|H_{I}\left(t^{\prime}\right)\right| 0_{\mathrm{ph}}\right\rangle \\
& \quad-\frac{1}{\hbar^{2}} \int_{t_{i-1}}^{t_{i}} \mathrm{~d} t^{\prime} \int_{t_{i-1}}^{t^{\prime}} \mathrm{d} t^{\prime \prime}\left\langle 0_{\mathrm{ph}}\left|H_{I}\left(t^{\prime}\right) H_{I}\left(t^{\prime \prime}\right)\right| 0_{\mathrm{ph}}\right\rangle+\mathcal{O}\left(\Delta t^{2}\right)
\end{aligned}
$$

with $H^{\mathrm{I}}(t)=\mathrm{e}^{\mathrm{i} H_{0} t / \hbar} H_{1} \mathrm{e}^{-\mathrm{i} H_{0} t / \hbar}$. It is important to note that $U_{\mathrm{c}}\left(t, t_{0}\right)$ is non-unitary, since the norm of the conditionally developed state diminishes in time and it equals the probability $N\left(t ; \boldsymbol{\Psi}_{0}\right)$ to find no photon until time $t$,

$$
N\left(t ; \mathbf{\Psi}_{0}\right)=\| U_{\mathrm{c}}(t, 0)\left|\boldsymbol{\Psi}_{0}\right\rangle\left\|^{2} \equiv\right\|\left|\boldsymbol{\Psi}_{t}\right\rangle \|^{2},
$$

if the initial state of the atom at time $t=0$ is $\left|\Psi_{0}\right\rangle$. The extension to density matrices is obvious: If $\rho_{0}$ denotes the atomic density matrix at $t=0$, then the conditional developed matrix is given by

$$
\rho(t)=U_{\mathrm{c}}(t, 0) \rho_{0} U_{\mathrm{c}}^{\dagger}(t, 0)
$$

and

$$
N\left(t ; \rho_{0}\right)=\operatorname{Tr} \rho(t) .
$$

The generator of the conditional time evolution is the non-Hermitian Hamiltonian $H_{\mathrm{c}}$ such that

$$
U_{\mathrm{c}}\left(t, t_{0}\right)=\mathrm{e}^{\mathrm{i} H_{\mathrm{c}}\left(t-t_{0}\right) / \hbar}
$$

holds.

\subsubsection{The reset operation and the Bloch equation}

In the following it is assumed that an emitted photon does not interact anymore with the atom [86]. Starting again with the state $\left|0_{\mathrm{ph}}\right\rangle \rho_{0}\left\langle 0_{\mathrm{ph}}\right|$ at time $t=0$, the state at time $\Delta t$ in the interaction picture is given by

$$
\rho(\Delta t)=U^{\mathrm{I}}(\Delta t, 0)\left|0_{\mathrm{ph}}\right\rangle \rho_{0}\left\langle 0_{\mathrm{ph}}\right| U^{\mathrm{I}}(\Delta t, 0)^{\dagger} .
$$


A subsequent detection of a photon due to the measurement corresponds to a projection onto the one-photon space by the projector $\widehat{P}_{1}=\sum_{\mathbf{k} \lambda}\left|1_{\mathbf{k} \lambda}\right\rangle \widehat{1}_{\mathrm{A}}\left\langle 1_{\mathbf{k} \lambda}\right|$, where the sum extends over all photon wave vectors $\mathbf{k}$ and polarizations $\lambda$. Physically, this is related to a state change (jump), and the atomic density matrix is reset to a new (unnormalized) density matrix, denoted by $\mathscr{R}\left(\rho_{0}\right)$, which is given by a partial trace over the one-photon space,

$$
\begin{aligned}
\mathscr{R}\left(\rho_{0}\right) \Delta t & =\operatorname{Tr}_{\mathrm{ph}}\left(\widehat{P}_{1} U^{\mathrm{I}}(\Delta t, 0)\left|0_{\mathrm{ph}}\right\rangle \rho_{0}\left\langle 0_{\mathrm{ph}}\right| U^{\mathrm{I}}(\Delta t, 0)^{\dagger} \widehat{P}_{1}\right) \\
& =\sum_{\mathbf{k} \lambda}\left\langle 1_{\mathbf{k} \lambda}\left|U^{\mathrm{I}}(\Delta t, 0)\right| 0_{\mathrm{ph}}\right\rangle \rho_{0}\left\langle 0_{\mathrm{ph}}\left|U^{\mathrm{I}}(\Delta t, 0)^{\dagger}\right| 1_{\mathbf{k} \lambda}\right\rangle .
\end{aligned}
$$

Here $\mathscr{R}$ is a super-operator that acts on density matrices. For the normalized reset state it follows that

$$
\mathscr{R}_{\mathrm{N}}\left(\rho_{0}\right)=\frac{\mathscr{R}\left(\rho_{0}\right)}{\operatorname{Tr} \mathscr{R}\left(\rho_{0}\right)},
$$

where $\operatorname{Tr} \mathscr{R}\left(\rho_{0}\right) \Delta t$ is the probability to detect a photon within the time interval $\Delta t$.

The two possible evolutions of an ensemble of atoms, described at time $t$ by $\rho(t)$, are related to the outcomes of the measurement at time $t+\Delta t$ : no photon detection or photon detection. The sub-ensemble that has not emitted a photon has to be described by a conditional time evolution, whereas the sub-ensemble, that has emitted a photon is given by the (unnormalized) reset state. For the complete ensemble one has

$$
\begin{aligned}
\rho(t+\Delta t) & =U_{\mathrm{c}}(t+\Delta t, t) \rho(t) U_{\mathrm{c}}^{\dagger}(t+\Delta t, t)+\mathscr{R}(\rho(t)) \Delta t \\
& =\left(\widehat{1}_{\mathrm{A}}-\frac{\mathrm{i}}{\hbar} H_{\mathrm{c}} \Delta t\right) \rho(t)\left(\widehat{1}_{\mathrm{A}}+\frac{\mathrm{i}}{\hbar} H_{\mathrm{c}}^{\dagger} \Delta t\right)+\mathscr{R}(\rho(t)) \Delta t+\mathcal{O}\left(\Delta t^{2}\right) \\
& =\rho(t)-\frac{\mathrm{i}}{\hbar}\left(H_{\mathrm{c}} \rho(t)-\rho(t) H_{\mathrm{c}}^{\dagger}\right) \Delta t+\mathscr{R}(\rho(t)) \Delta t+\mathcal{O}\left(\Delta t^{2}\right) .
\end{aligned}
$$

Since $\Delta t$ is much smaller than the time scale on which $\rho(t)$ evolves, it follows

$$
\dot{\rho}(t)=-\frac{\mathrm{i}}{\hbar}\left(H_{\mathrm{c}} \rho(t)-\rho(t) H_{\mathrm{c}}^{\dagger}\right)+\mathscr{R}(\rho(t)) .
$$

This is the general master equation for the time evolution of an atom, interacting with a radiation field, and it is known as the optical Bloch equation. It has been shown [86] that this master equation is equivalent to that obtained by Agarwal [92] who used a different approach. Another notation can be introduced by means of the Liouville super-operator $\mathscr{L}$, defined by

$$
\dot{\rho}(t)=\mathscr{L} \rho(t)
$$

which leads to the fact that the formal solution of the Bloch equation can be written as

$$
\rho(t)=\mathrm{e}^{\mathscr{L} t} \rho_{0}
$$




\subsubsection{Exclusive and non-exclusive detection probability}

In Eq. (3.8), an expression for the probability of measuring no photon until time $t$ has been derived. It follows that $N\left(t ; \rho_{0}\right)-N\left(t+\Delta t ; \rho_{0}\right)$ is the probability to observe the first photon in the interval $(t, t+\Delta t)$ and the corresponding probability density is given by

$$
w_{1}\left(t ; \rho_{0}\right)=-\frac{\mathrm{d}}{\mathrm{d} t} N\left(t ; \rho_{0}\right) .
$$

Since there are no photons in between, it is named "exclusive" detection probability. In contrast, one may ask for the probability density to detect a photon at time $t$, irrespective of previous detections. This "non-exclusive" probability density is denoted by $I\left(t ; \rho_{0}\right)$ and can be derived by the following two methods:

Derivation of $I\left(t ; \rho_{0}\right)$ via integral equation: The probability density of an photon emission at time $t$ can be composed by two contributions: One is related to the possibility that the emission is the first one, described by $w_{1}\left(t ; \rho_{0}\right)$, and the second is related to the probability that the emission is the first one after a foregoing one at time $t^{\prime}<t$. Adding up these probabilities, the resulting density is

$$
I\left(t ; \rho_{0}\right)=w_{1}\left(t ; \rho_{0}\right)+\int_{0}^{t} \mathrm{~d} t^{\prime} I\left(t^{\prime} ; \rho_{0}\right) w_{1}\left(t-t^{\prime} ; \mathscr{R}\left(\rho\left(t^{\prime}\right)\right)\right) .
$$

This Volterra integral equation can be easily solved, if the reset state $\mathscr{R}(\rho(t))$ is equal to $\rho_{0}$ for all $t$, since then a convolution type kernel arises and $I\left(t ; \rho_{0}\right)$ is obtained via Laplace transformation. An example for that case is a laser-illuminated two-level atom at rest.

Derivation of $I\left(t ; \rho_{0}\right)$ via Bloch equation: According to the definition of $I\left(t ; \rho_{0}\right)$, the probability of detecting a photon in the time interval $(t, t+\Delta t)$ is given by $I\left(t ; \rho_{0}\right) \Delta t$ and can be expressed by means of the probability, to measure no photon in that interval, i.e.

$$
I\left(t ; \rho_{0}\right) \Delta t=1-N(t+\Delta t ; \rho(t)) .
$$

If no photon is observed, the time evolution of the atom in the interval $(t, t+\Delta t)$ takes place with the conditional Hamiltonian, which means

$$
\begin{aligned}
N(t+\Delta t ; \rho(t)) & =\operatorname{Tr}\left(\mathrm{e}^{-\mathrm{i} H_{\mathrm{c}} \Delta t / \hbar} \rho(t) \mathrm{e}^{\mathrm{i} H_{\mathrm{c}}^{\dagger} \Delta t / \hbar}\right) \\
& =1-\frac{\mathrm{i}}{\hbar} \operatorname{Tr}\left(\left(H_{\mathrm{c}}-H_{\mathrm{c}}^{\dagger}\right) \rho(t)\right) \Delta t+\mathcal{O}\left(\Delta t^{2}\right) .
\end{aligned}
$$

By comparing Eq. (3.19) and Eq. (3.20) one obtains the result

$$
I\left(t ; \rho_{0}\right)=\frac{\mathrm{i}}{\hbar} \operatorname{Tr}\left(\left(H_{\mathrm{c}}-H_{\mathrm{c}}^{\dagger}\right) \rho(t)\right) .
$$

Clearly, the time-developed density matrix $\rho(t)$ has to be known to derive $I\left(t ; \rho_{0}\right)$. 


\subsubsection{Example: Two-level system with quantized $\mathrm{cm}$ motion}

As an important example that will provide the necessary expressions for the operational model to arrival times, this section treats a moving two-level atom with quantized $\mathrm{cm}$ motion, interacting with a classical laser field $\mathbf{E}_{\mathrm{L}}$ and the quantized radiation field $\widehat{\mathbf{E}}$ [87]. The position and the momentum operator for the center-ofmass are denoted by $\widehat{\mathbf{x}}$ and $\widehat{\mathbf{p}}$, respectively. The corresponding Hamiltonian in the Schrödinger picture reads

$$
H=\frac{\widehat{\mathbf{p}}^{2}}{2 m}+\hbar \omega_{21}|2\rangle\langle 2|+\sum_{\mathbf{k} \lambda} \hbar \omega_{k} a_{\mathbf{k} \lambda}^{\dagger} a_{\mathbf{k} \lambda}+\widehat{\mathbf{D}} \cdot \widehat{\mathbf{E}}(\widehat{\mathbf{x}}, 0)+\widehat{\mathbf{D}} \cdot \mathbf{E}_{\mathrm{L}}(\widehat{\mathbf{x}}, t),
$$

where $\hbar \omega_{21}$ is the energy difference between the ground state $|1\rangle$ and the excited state $|2\rangle, a_{\mathbf{k} \lambda}\left(a_{\mathbf{k} \lambda}^{\dagger}\right)$ are the lowering (raising) operators for the field mode with wave vector $\mathbf{k}$ and polarization $\lambda$ and $\omega_{k}=c|\mathbf{k}|=c k$. The interaction is given by the dipole moment of the transition,

$$
\widehat{\mathbf{D}}=\mathbf{D}_{12}|1\rangle\left\langle 2\left|+\mathbf{D}_{12}^{*}\right| 2\right\rangle\langle 1|,
$$

where $\mathbf{D}_{12}=e\langle 1|\widehat{\mathbf{r}}| 2\rangle$ is the dipole matrix element of the atom and $e$ is the elementary charge, and the field terms

$$
\widehat{\mathbf{E}}(\widehat{\mathbf{x}}, 0)=\mathrm{i} \sum_{\mathbf{k} \lambda} \sqrt{\frac{\hbar \omega_{k}}{2 \epsilon_{0} V}}\left(\epsilon_{\mathbf{k} \lambda} a_{\mathbf{k} \lambda} \mathrm{e}^{\mathrm{i} \mathbf{k} \cdot \widehat{\mathbf{x}}}-\epsilon_{\mathbf{k} \lambda}^{*} a_{\mathbf{k} \lambda}^{\dagger} \mathrm{e}^{-\mathbf{i k} \cdot \widehat{\mathbf{x}}}\right)
$$

and

$$
\mathbf{E}_{\mathrm{L}}(\widehat{\mathbf{x}}, t)=\operatorname{Re} \mathbf{E}_{0}(\widehat{\mathbf{x}}) \mathrm{e}^{\mathrm{i}\left(\mathbf{k}_{\mathrm{L}} \cdot \widehat{\mathbf{x}}-\omega_{\mathrm{L}} t\right)} .
$$

In the laser-adapted interaction picture with respect to $H_{0}=\widehat{\mathbf{p}}^{2} / 2 m+\hbar \omega_{\mathrm{L}}|2\rangle\langle 2|+$ $\sum_{\mathbf{k} \lambda} \hbar \omega_{k} a_{\mathbf{k} \lambda}^{\dagger} a_{\mathbf{k} \lambda}$ and within the rotating-wave approximation the Hamiltonian reads

$$
\begin{aligned}
H^{\mathrm{I}}(t)=-\hbar \delta|2\rangle\langle 2|+\mathrm{e}^{\mathrm{i} \widehat{\mathbf{p}}^{2} t / 2 m \hbar}\left[\sum_{\mathbf{k} \lambda}(\right. & \left.\hbar g_{\mathbf{k} \lambda} a_{\mathbf{k} \lambda}|2\rangle\langle 1| \mathrm{e}^{\mathrm{i} \mathbf{k} \cdot \widehat{\mathbf{x}}} \mathrm{e}^{\mathrm{i}\left(\omega_{\mathrm{L}}-\omega_{k}\right) t}+\text { h.c. }\right) \\
+ & \left.\frac{\hbar}{2} \Omega(\widehat{\mathbf{x}})\left(|2\rangle\langle 1| \mathrm{e}^{\mathrm{i} \mathbf{k}_{\mathrm{L}} \cdot \widehat{\mathbf{x}}}+\text { h.c. }\right)\right] \mathrm{e}^{-\mathrm{i} \widehat{\mathbf{p}}^{2} t / 2 m \hbar},
\end{aligned}
$$

where the detuning

$$
\delta=\omega_{\mathrm{L}}-\omega_{21},
$$

the Rabi frequency

$$
\Omega(\widehat{\mathbf{x}})=\mathbf{D}_{12} \cdot \mathbf{E}_{0}(\widehat{\mathbf{x}})
$$

and the coupling constant

$$
g_{\mathbf{k} \lambda}=\mathrm{i} \sqrt{\frac{\omega_{k}}{2 \epsilon_{0} \hbar V}}\left(\mathbf{D}_{21} \cdot \epsilon_{\mathbf{k} \lambda}\right)
$$

have been introduced. To proceed, one notes that the action of $\mathrm{e}^{ \pm \hat{\mathbf{p}}^{2} t / 2 m \hbar}$ on the operator $\widehat{\mathbf{x}}$ corresponds to the time development of $\widehat{\mathbf{x}}$ in the free Heisenberg picture, i.e.

$$
\mathrm{e}^{\mathrm{i} \widehat{\mathbf{p}}^{2} t / 2 m \hbar} \widehat{\mathbf{x}} \mathrm{e}^{-\mathrm{i} \widehat{\mathbf{p}}^{2} t / 2 m \hbar}=\widehat{\mathbf{x}}+\widehat{\mathbf{p}} t / m \equiv \widehat{\mathbf{x}}(t)
$$


Chapter 3. Operational approaches to quantum arrival times

Inserting Eq. (3.26) into Eq. (3.5) yields the conditional time evolution for the twolevel system, where only terms of the order of $\Delta t$ are kept. The laser part solely contributes to the first order of the Dyson series, whereas the second order is given by

$$
-\int_{t_{i-1}}^{t_{i}} \mathrm{~d} t^{\prime} \int_{t_{i-1}}^{t^{\prime}} \mathrm{d} t^{\prime \prime} \sum_{\mathbf{k} \lambda}\left|g_{\mathbf{k} \lambda}\right|^{2} \mathrm{e}^{\mathrm{i} \mathbf{k} \cdot\left(\widehat{\mathbf{x}}+\widehat{\mathbf{p}} t^{\prime} / m\right)} \mathrm{e}^{-\mathrm{i} \mathbf{k} \cdot\left(\widehat{\mathbf{x}}+\widehat{\mathbf{p}} t^{\prime \prime} / m\right)} \mathrm{e}^{\mathrm{i}\left(\omega_{\mathrm{L}}-\omega_{k}\right)\left(t^{\prime}-t^{\prime \prime}\right)}|2\rangle\langle 2| .
$$

If $t^{\prime}, t^{\prime \prime}$ is of the order of $\Delta t$ and the atom is not moving too fast, one may assume the approximation

$$
\mathbf{x}\left(t^{\prime}\right) \approx \mathbf{x}\left(t^{\prime \prime}\right)
$$

to be valid [87]. Consequences of the neglected terms are discussed in the following section. With Eq. (3.32), two exponential functions in Eq. (3.31) cancel each other and the resulting expression is the same as for an atom at rest, which has been derived in Ref. [86], leading to

$$
\frac{1}{\hbar^{2}} \int_{t_{i-1}}^{t_{i}} \mathrm{~d} t^{\prime} \int_{t_{i-1}}^{t^{\prime}} \mathrm{d} t^{\prime \prime}\left\langle 0_{\mathrm{ph}}\left|H_{I}\left(t^{\prime}\right) H_{I}\left(t^{\prime \prime}\right)\right| 0_{\mathrm{ph}}\right\rangle=-\frac{\gamma}{2}|2\rangle\langle 2| \Delta t,
$$

where $\gamma$ is the Einstein coefficient of the transition $|2\rangle \rightarrow|1\rangle$,

$$
\gamma=\frac{e^{2} \omega_{21}^{3}\left|\mathbf{D}_{21}\right|^{2}}{3 \pi \epsilon_{0} \hbar c^{3}}
$$

For the conditional time-development operator follows

$$
\begin{aligned}
& U_{\mathrm{c}}^{\mathrm{I}}\left(t_{i}, t_{i-1}\right)= \widehat{1}_{\mathrm{A}}-\frac{\mathrm{i}}{\hbar} \int_{t_{i-1}}^{t_{i}} \mathrm{~d} t^{\prime}[-\hbar \delta|2\rangle\langle 2| \\
&\left.+\frac{\hbar \Omega\left(\widehat{\mathbf{x}}+\widehat{\mathbf{p}} t^{\prime} / m\right)}{2}\left(|2\rangle\langle 1| \mathrm{e}^{\mathrm{i} \mathbf{k}_{\mathrm{L}} \cdot\left(\widehat{\mathbf{x}}+\widehat{\mathbf{p}} t^{\prime} / m\right)}+\text { h.c. }\right)\right]-\frac{\gamma}{2}|2\rangle\langle 2| \Delta t \\
&=\widehat{1}_{\mathrm{A}}-\frac{\mathrm{i}}{\hbar} \int_{t_{i-1}}^{t_{i}} \mathrm{~d} t^{\prime}\left[-\frac{\hbar}{2}(\mathrm{i} \gamma+2 \delta)|2\rangle\langle 2|\right. \\
&\left.\quad+\frac{\hbar \Omega\left(\widehat{\mathbf{x}}+\widehat{\mathbf{p}} t^{\prime} / m\right)}{2}\left(|2\rangle\langle 1| \mathrm{e}^{\mathrm{i} \mathbf{k}_{\mathrm{L}} \cdot\left(\widehat{\mathbf{x}}+\widehat{\mathbf{p}} t^{\prime} / m\right)}+\text { h.c. }\right)\right] .
\end{aligned}
$$

The corresponding conditional Hamiltonian reads

$$
H_{\mathrm{c}}^{\mathrm{I}}(t)=\frac{\hbar \Omega(\widehat{\mathbf{x}}+\widehat{\mathbf{p}} t / m)}{2}\left(|2\rangle\langle 1| \mathrm{e}^{\mathrm{i} \mathbf{k}_{\mathrm{L}} \cdot(\widehat{\mathbf{x}}+\widehat{\mathbf{p}} t / m)}+\text { h.c. }\right)-\frac{\hbar}{2}(\mathrm{i} \gamma+2 \delta)|2\rangle\langle 2| .
$$

One can get rid off the time dependency by going out of the $\mathrm{cm}$-adapted interaction picture, leading to a different interaction picture with respect to the internal free Hamiltonian,

$$
\begin{aligned}
H_{\mathrm{c}}^{\mathrm{I} 2} & =\frac{\widehat{\mathbf{p}}^{2}}{2 m}+\mathrm{e}^{-\mathrm{i} \widehat{\mathbf{p}}^{2} t / 2 m \hbar} H_{\mathrm{c}}^{\mathrm{I}}(t) \mathrm{e}^{\mathrm{i} \widehat{\mathbf{p}}^{2} t / 2 m \hbar} \\
& =\frac{\widehat{\mathbf{p}}^{2}}{2 m}+\frac{\hbar \Omega(\widehat{\mathbf{x}})}{2}\left(|2\rangle\langle 1| \mathrm{e}^{\mathrm{i} \mathbf{k}_{\mathrm{L}} \cdot \widehat{\mathbf{x}}}+\text { h.c. }\right)-\frac{\hbar}{2}(\mathrm{i} \gamma+2 \delta)|2\rangle\langle 2| .
\end{aligned}
$$


A third interaction picture can be obtained from Eq. (3.36) by going out of the internal-state-adapted interaction picture,

$$
\begin{aligned}
H_{\mathrm{c}}^{\mathrm{I} 3} & =\hbar \omega_{\mathrm{L}}|2\rangle\langle 2|+\mathrm{e}^{-\mathrm{i} \omega_{\mathrm{L}} t|2\rangle\langle 2|} H_{\mathrm{c}}^{\mathrm{I}}(t) \mathrm{e}^{\mathrm{i} \hbar \omega_{\mathrm{L}} t|2\rangle\langle 2|} \\
& =\hbar \omega_{\mathrm{L}}|2\rangle\langle 2|+\frac{\hbar}{2} \Omega(\widehat{\mathbf{x}})\left(|2\rangle\langle 1| \mathrm{e}^{\mathrm{i} \mathbf{k}_{\mathrm{L}} \cdot \widehat{\mathbf{x}}-\mathrm{i} \omega_{\mathrm{L}}(\widehat{\mathbf{v}}) t}+\text { h.c. }\right)-\frac{\hbar}{2}(\mathrm{i} \gamma+2 \delta)|2\rangle\langle 2|,
\end{aligned}
$$

where

$$
\omega_{\mathrm{L}}(\widehat{\mathbf{v}})=\omega_{\mathrm{L}}\left(1-\frac{\widehat{\mathbf{v}} \cdot \mathbf{e}_{\mathrm{L}}}{c}\right)
$$

is the laser frequency seen from the atom including the Doppler effect and $\mathbf{e}_{\mathrm{L}}=$ $\mathbf{k}_{\mathrm{L}} / k_{\mathrm{L}}$ is the unit vector of the laser direction.

Next, the reset matrix is considered which describes the $\mathrm{cm}$ motion and the internal degrees of freedom right after the detection of a photon. Taking matrix elements with the momentum eigenvectors,

$$
\rho_{t}\left(\mathbf{p}, \mathbf{p}^{\prime}\right)=\left\langle\mathbf{p}|\rho(t)| \mathbf{p}^{\prime}\right\rangle
$$

and inserting the Dyson series for $U^{\mathrm{I}}(\Delta t, 0)$ into Eq. (3.11) yields, following the derivation of Ref. [86] (note the additional factor $3 / 8 \pi$ which is missing there),

$$
\begin{aligned}
& \left\langle\mathbf{p}\left|\mathscr{R}\left(\rho_{t}\right)\right| \mathbf{p}^{\prime}\right\rangle \\
& \quad=\gamma|1\rangle\langle 1| \int \mathrm{d} \Omega_{\mathbf{k}} \frac{3}{8 \pi}\left(1-\frac{\left|\mathbf{e}_{k} \cdot \mathbf{D}_{21}\right|^{2}}{\left|\mathbf{D}_{21}\right|^{2}}\right)\left\langle 2\left|\rho_{t}\left(\mathbf{p}+\hbar \omega_{21} \mathbf{e}_{k} / c, \mathbf{p}^{\prime}+\hbar \omega_{21} \mathbf{e}_{k} / c\right)\right| 2\right\rangle
\end{aligned}
$$

where $\mathbf{e}_{k}=\mathbf{k} / k$ and integration runs over the unit sphere $k=1$. The momentum transfer from the spontaneously emitted photon onto the atom is included in Eq. (3.41), which represents the contrast to the case of an atom at rest. It is interesting to note that the trace of this reset state is independent of the recoil, since

$$
\begin{aligned}
\operatorname{Tr}\left\langle\mathbf{p}\left|\mathscr{R}\left(\rho_{t}\right)\right| \mathbf{p}^{\prime}\right\rangle= & \gamma \int \mathrm{d} \Omega_{\mathbf{k}} \frac{3}{8 \pi}\left(1-\frac{\left|\mathbf{e}_{k} \cdot \mathbf{D}_{21}\right|^{2}}{\left|\mathbf{D}_{21}\right|^{2}}\right) \\
& \times \int_{-\infty}^{\infty} \mathrm{d}^{3} p\left\langle 2\left|\rho_{t}\left(\mathbf{p}+\hbar \omega_{21} \mathbf{e}_{k} / c, \mathbf{p}+\hbar \omega_{21} \mathbf{e}_{k} / c\right)\right| 2\right\rangle \\
& =\gamma \int \mathrm{d} \Omega_{\mathbf{k}} \frac{3}{8 \pi}\left(1-\frac{\left|\mathbf{e}_{k} \cdot \mathbf{D}_{21}\right|^{2}}{\left|\mathbf{D}_{21}\right|^{2}}\right) \int_{-\infty}^{\infty} \mathrm{d}^{3} p\left|\widetilde{\psi}_{t}^{(2)}\left(\mathbf{p}+\hbar \omega_{21} \mathbf{e}_{k} / c\right)\right|^{2} \\
& =\gamma \int \mathrm{d} \Omega_{\mathbf{k}} \frac{3}{8 \pi}\left(1-\frac{\left|\mathbf{e}_{k} \cdot \mathbf{D}_{21}\right|^{2}}{\left|\mathbf{D}_{21}\right|^{2}}\right) \int_{-\infty}^{\infty} \mathrm{d}^{3} x\left|\psi_{t}^{(2)}(\mathbf{x})\right|^{2} \\
& =\gamma \int_{-\infty}^{\infty} \mathrm{d}^{3} x\left|\psi_{t}^{(2)}(\mathbf{x})\right|^{2}
\end{aligned}
$$

where Parseval's theorem has been used in Eq. (3.44) and $\int \mathrm{d} \Omega_{\mathbf{k}}\left(1-\frac{\left|\mathbf{e}_{k} \cdot \mathbf{D}_{21}\right|^{2}}{\left|\mathbf{D}_{21}\right|^{2}}\right)=$ $2 \pi \int_{0}^{\pi} \mathrm{d} \vartheta\left(1-\cos ^{2} \vartheta\right)=8 \pi / 3$.

The reset state for one-dimensional atomic motion can be obtained from Eq. (3.41) by taking the trace over the momentum components $p_{y}$ and $p_{z}$ [83]. 


\subsubsection{Corrections to the conditional time evolution}

In the previous section it has been shown that within the framework of the approximation (3.32) the longitudinal Doppler effect, an effect of the order of $v / c$, is included in the present approach. To obtain possible other motion-dependent effects in the conditional time evolution, Eq. (3.31) is investigated in the following without assuming (3.32).

Using the standard relation

$$
\mathrm{e}^{\widehat{A}} \mathrm{e}^{\widehat{B}}=\mathrm{e}^{\widehat{A}+\widehat{B}} \mathrm{e}^{[\widehat{A}, \widehat{B}] / 2}
$$

valid for operators which commute with their commutator, Eq. (3.31) becomes

$$
-\int_{t_{i-1}}^{t_{i}} \mathrm{~d} t^{\prime} \int_{t_{i-1}}^{t^{\prime}} \mathrm{d} t^{\prime \prime} \sum_{\mathbf{k} \lambda}\left|g_{\mathbf{k} \lambda}\right|^{2} \mathrm{e}^{\mathrm{i}\left(\mathbf{k} \cdot \widehat{\mathbf{v}}-E_{k} / \hbar+\omega_{\mathrm{L}}-\omega_{k}\right)\left(t^{\prime}-t^{\prime \prime}\right)}|2\rangle\langle 2|,
$$

where $\widehat{\mathbf{v}}=\widehat{\mathbf{p}} / m$ and $E_{k}=\hbar^{2} k^{2} / 2 m$ has been defined for convenience. Since the corrections are small compared to $\omega_{\mathrm{L}}-\omega_{k}$, the derivation of Ref. [86] can be adopted and for the expression (3.47) this leads to

$$
-\frac{e^{2}\left|\mathbf{D}_{12}\right|^{2}}{16 \pi^{2} \epsilon_{0} \hbar c^{3}}|2\rangle\langle 2| \Delta t \int_{0}^{\infty} \mathrm{d} \omega_{k} \omega_{k}^{3} \int \mathrm{d} \Omega_{\mathbf{k}}\left(1-\frac{\left|\mathbf{e}_{k} \cdot \mathbf{D}_{21}\right|^{2}}{\left|\mathbf{D}_{21}\right|^{2}}\right) \delta\left(\mathbf{k} \cdot \widehat{\mathbf{v}}-E_{k} / \hbar+\omega_{\mathrm{L}}-\omega_{k}\right) .
$$

In the following, the derivation is performed in the eigenbasis of $\widehat{\mathbf{v}}$, where one has $\widehat{\mathbf{v}}|\mathbf{v}\rangle=\mathbf{v}|\mathbf{v}\rangle$ and the evaluation of the Dirac delta function thus reads

$$
\delta\left(\mathbf{k} \cdot \mathbf{v}-E_{k} / \hbar+\omega_{\mathrm{L}}-\omega_{k}\right)=\frac{2 m c^{2}}{\hbar} \delta\left(\omega_{k}^{2}+\frac{2 m c^{2}}{\hbar} \omega_{k}\left(1-\frac{\mathbf{v} \cdot \mathbf{e}_{k}}{c}\right)-\frac{2 m c^{2}}{\hbar} \omega_{\mathrm{L}}\right) .
$$

The only positive zero of the argument of the delta function is

$$
\omega_{k, 0}=-\frac{m c^{2}}{\hbar}\left[\left(1-\frac{\mathbf{v} \cdot \mathbf{e}_{k}}{c}\right)-\sqrt{\left(1-\frac{\mathbf{v} \cdot \mathbf{e}_{k}}{c}\right)^{2}+\frac{2 \hbar \omega_{\mathrm{L}}}{m c^{2}}}\right] .
$$

For optical frequencies and the atomic mass of a cesium atom, $m \sim 10^{-25} \mathrm{~kg}$, one has $\hbar \omega_{\mathrm{L}} / m c^{2} \sim 10^{-10} \ll 1$ and the root can be expanded, leading to

$$
\omega_{k, 0} \simeq \frac{\omega_{\mathrm{L}}}{1-\mathbf{v} \cdot \mathbf{e}_{k} / c}
$$

In a similar way the derivative of the argument of the delta function evaluated at $\omega_{k, 0}$ becomes $2 m c^{2}\left(1-\mathbf{v} \cdot \mathbf{e}_{k} / c\right) / \hbar$, such that

$$
\delta\left(\mathbf{k} \cdot \widehat{\mathbf{v}}-E_{k} / \hbar+\omega_{\mathrm{L}}-\omega_{k}\right)|\mathbf{v}\rangle \simeq \frac{\delta\left(\omega_{k}-\frac{\omega_{\mathrm{L}}}{1-\mathbf{v} \cdot \mathbf{e}_{k} / c}\right)}{1-\mathbf{v} \cdot \mathbf{e}_{k} / c}|\mathbf{v}\rangle
$$


To evaluate the integration over the unit sphere of $\mathbf{k}$, the dipole moment $\mathbf{D}_{12}$ is chosen as real and pointing in the $z$-direction. Inserting Eq. (3.52) into Eq. (3.48), changing the integration order, and integrating over $\omega_{k}$ yields

$$
\begin{aligned}
-\frac{e^{2}\left|\mathbf{D}_{12}\right|^{2} \omega_{\mathrm{L}}^{3}}{16 \pi^{2} \epsilon_{0} \hbar c^{3}}|2\rangle\langle 2| \Delta t \int_{0}^{2 \pi} \mathrm{d} \varphi & \int_{0}^{\pi} \mathrm{d} \vartheta \sin \vartheta \frac{1-\cos ^{2} \vartheta}{\left(1-\mathbf{v} \cdot \mathbf{e}_{\mathbf{k}} / c\right)^{4}}|\mathbf{v}\rangle \\
=-\frac{e^{2}\left|\mathbf{D}_{12}\right|^{2} \omega_{\mathrm{L}}^{3}}{16 \pi^{2} \epsilon_{0} \hbar c^{3}}|2\rangle & \langle 2| \Delta t \int_{0}^{2 \pi} \mathrm{d} \varphi \int_{0}^{\pi} \mathrm{d} \vartheta \sin \vartheta\left(1-\cos ^{2} \vartheta\right) \\
& \times\left(1+4 \frac{\mathbf{v} \cdot \mathbf{e}_{k}}{c}+10 \frac{\left(\mathbf{v} \cdot \mathbf{e}_{k}\right)^{2}}{c^{2}}+\mathcal{O}\left(\frac{v^{3}}{c^{3}}\right)\right)|\mathbf{v}\rangle .
\end{aligned}
$$

Now $\mathbf{v}$ can be assumed to be in the $x z$-plane, such that $\mathbf{v}=v\left(\sin \vartheta_{v}, 0, \cos \vartheta_{v}\right)$ and $\mathbf{v} \cdot \mathbf{e}_{k}=v\left(\sin \vartheta_{v} \sin \vartheta \cos \varphi+\cos \vartheta_{v} \cos \vartheta\right)$, where $\vartheta_{v}$ is the angle between $\mathbf{v}$ and $\mathbf{D}_{12}$. After performing the angle integration, the correction of Eq. (3.33) is obtained as

$$
\begin{aligned}
\frac{1}{\hbar^{2}} \int_{t_{i-1}}^{t_{i}} \mathrm{~d} t^{\prime} \int_{t_{i-1}}^{t^{\prime}} \mathrm{d} t^{\prime \prime} & \left\langle 0_{\mathrm{ph}}\left|H_{I}\left(t^{\prime}\right) H_{I}\left(t^{\prime \prime}\right)\right| 0_{\mathrm{ph}}\right\rangle|\mathbf{v}\rangle \\
& =\left\{-\frac{\gamma}{2}\left(1+2 \frac{v^{2}}{c^{2}}\left(1+\sin ^{2} \vartheta_{v}\right)\right)|2\rangle\langle 2| \Delta t+\mathcal{O}\left(\frac{v^{4}}{c^{4}}\right)\right\}|\mathbf{v}\rangle
\end{aligned}
$$

The additional factor is not the true relativistic correction of the Einstein coefficient $\gamma$, since the lifetime of a moving atom becomes larger and $\gamma$ should become smaller. But this is not expected, since all considerations rely on the non-relativistic Schrödinger equation where only the first order term in the kinetic energy, $\mathbf{p}^{2} / 2 m$, is included in Eq. (3.22). What has been shown is that the disregard of Eq. (3.32) leads to terms of the order of $v^{2} / c^{2}$ which can be neglected for atoms which are not moving too fast. Note that in the derivation of the laser term in Eq. (3.36) the assumption (3.32) has not been used and therefore this term has not to be considered.

\subsection{Laser-based approach to quantum arrival-time distributions}

In this section, the central operational model to quantum arrival times is presented. The basic idea is described in the following [24]. One is interested in the arrivaltime distribution at the point $x_{\mathrm{A}}$ of a quantum particle, described by a wave packet $\psi(x, t)$. Throughout this work one-dimensional atomic motion is considered. This one-dimensional description is accurate for atoms traveling in waveguides [93, 94] and it is a very good approximation of free wave packets which are broad in the laser direction, perpendicular to the incident atomic motion [95]. Unless otherwise noted, $x_{\mathrm{A}}=0$ is assumed without loss of generality. The arrival-time distribution is modeled by the temporal distribution of the first photon emitted from a two-level atom (see Fig. 3.1a), where the atom is initially in the ground state and impinges on a laser field which is located in the right half-space $x \geq 0$. The laser field is assumed 
(a)

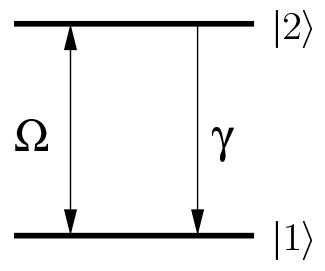

(b)

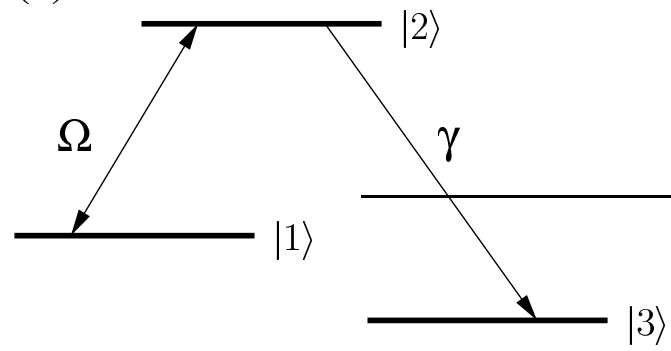

Figure 3.1: (a) Two-level system and (b) three-level system under consideration in this work.

Moving atom

= wave packet

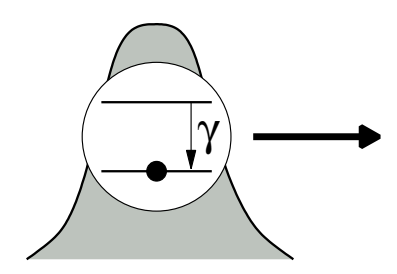

Laser field, perpendicular to the atom's motion, Rabi frequency $\Omega$

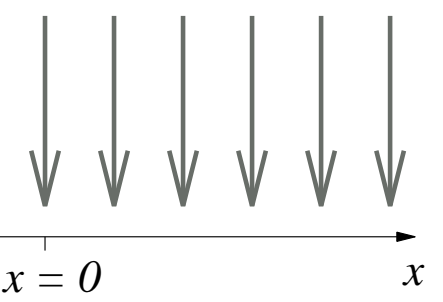

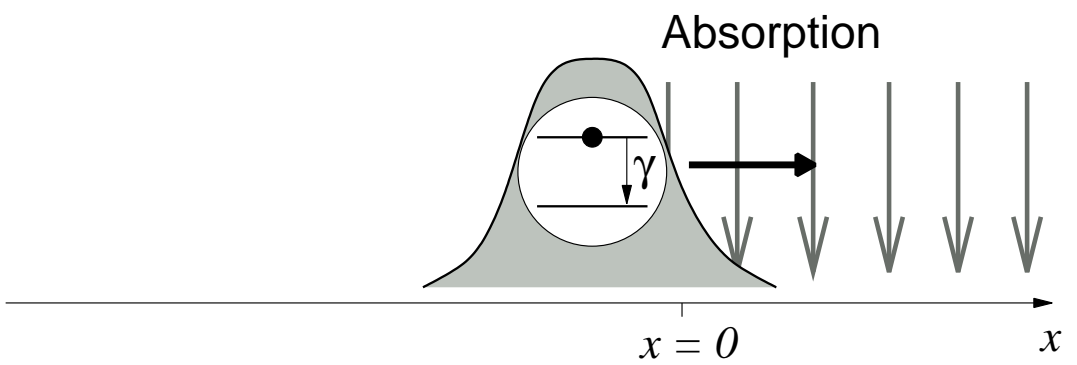

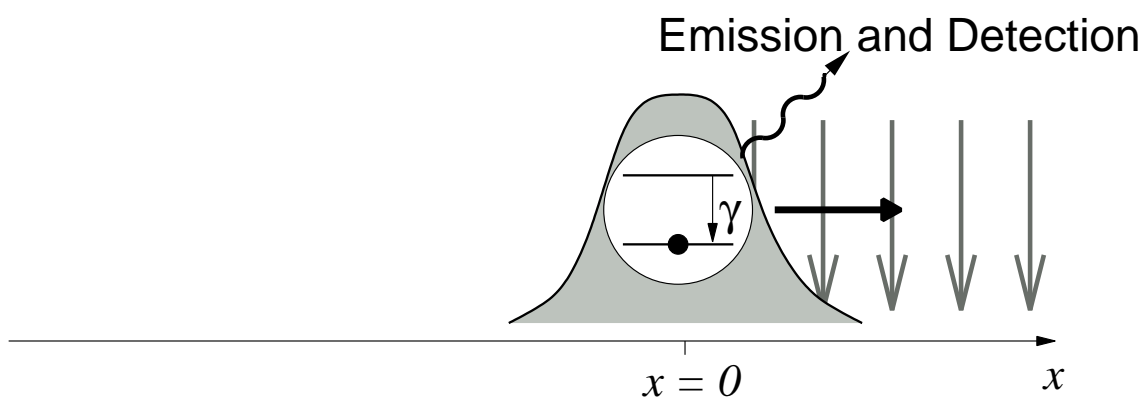

Figure 3.2: Schematical picture of the basic operational model for the measurement of an arrival-time distribution at $x_{\mathrm{A}}=0$ [24]. Note the occurrence of the delay with respect to excitation and decay. Shown is also the spreading of the wave packet. 
to be perpendicular to the atomic motion. A schematical picture for this model is given in Fig. 3.2.

Naturally, due to the mean times needed for excitation and decay, this firstphoton distribution is no ideal arrival-time distribution. But it will be shown in detail that there are limits, for which the operational distribution is related to ideal arrival-time distributions.

\subsubsection{The probability density for the first photon}

The conditional Hamiltonian (3.37) for one-dimensional motion and for a resonant laser field which is perpendicular to the motion and located in the right half-space $x \geq 0$ has the form

$$
H_{\mathrm{c}}=\frac{\widehat{p}^{2}}{2 m}+\frac{\hbar}{2}\left(\begin{array}{cc}
0 & \Omega \Theta(\widehat{x}) \\
\Omega \Theta(\widehat{x}) & -\mathrm{i} \gamma
\end{array}\right)
$$

where $\Theta(x)$ is the Heaviside step function, $|1\rangle \equiv\left(\begin{array}{l}1 \\ 0\end{array}\right)$ and $|2\rangle \equiv\left(\begin{array}{l}0 \\ 1\end{array}\right)$. To obtain the time development of a general wave packet until the first-photon detection, one has to solve the eigenvalue equation

$$
H_{\mathrm{c}} \boldsymbol{\Phi}_{k}=E_{k} \boldsymbol{\Phi}_{k}, \text { where } \boldsymbol{\Phi}_{k}=\left(\begin{array}{c}
\phi_{k}^{(1)} \\
\phi_{k}^{(2)}
\end{array}\right)
$$

for positive eigenvalues $E_{k}=\hbar^{2} k^{2} / 2 m>0$. Following the transfer matrix method for two-channel scattering problems that is given in Appendix B.2, the resulting eigenfunctions for an initial ground state plane wave coming in from the left are given by

$$
\Phi_{k}(x)=\frac{1}{\sqrt{2 \pi}} \begin{cases}\left(\begin{array}{c}
\mathrm{e}^{\mathrm{i} k x}+R_{1} \mathrm{e}^{-\mathrm{i} k x} \\
R_{2} \mathrm{e}^{-\mathrm{i} q x}
\end{array}\right), & x \leq 0, \\
C_{+}\left(\begin{array}{c}
1 \\
2 \lambda_{+} / \Omega
\end{array}\right) \mathrm{e}^{\mathrm{i} k_{+} x}+C_{-}\left(\begin{array}{c}
1 \\
2 \lambda_{-} / \Omega
\end{array}\right) \mathrm{e}^{\mathrm{i} k_{-} x}, & x \geq 0,\end{cases}
$$

where

$$
\begin{aligned}
q & =\sqrt{k^{2}+\mathrm{i} m \gamma / \hbar}, \quad \operatorname{Im} q>0, \\
\lambda_{ \pm} & =-\frac{\mathrm{i}}{4}\left(\gamma \mp \sqrt{\gamma^{2}-4 \Omega^{2}}\right), \\
k_{ \pm} & =\sqrt{k^{2}-2 m \lambda_{ \pm} / \hbar}, \quad \operatorname{Im} k_{ \pm}>0 .
\end{aligned}
$$

The amplitudes $R_{1}, R_{2}, C_{+}$and $C_{-}$are given by the matching conditions at $x=0$. Solving Eqs. (B.37) - (B.40), one has

$$
\begin{aligned}
& R_{1}=\left(\lambda^{+}\left(q+k^{+}\right)\left(k-k^{-}\right)-\lambda^{-}\left(q+k^{-}\right)\left(k-k^{+}\right)\right) / D, \\
& R_{2}=k\left(k^{-}-k^{+}\right) \Omega / D \\
& C_{+}=-2 k\left(q+k^{-}\right) \lambda^{-} / D \\
& C_{-}=2 k\left(q+k^{+}\right) \lambda^{+} / D
\end{aligned}
$$


with the common denominator

$$
D=\left(k+k^{-}\right)\left(q+k^{+}\right) \lambda^{+}-\left(k+k^{+}\right)\left(q+k^{-}\right) \lambda^{-} .
$$

The conditional time development of an initial ground-state wave packet $\left(\begin{array}{c}\psi\left(x, t_{0}\right) \\ 0\end{array}\right)$, $t_{0} \rightarrow-\infty$, is obtained by its decomposition as a superposition of energy eigenfunctions,

$$
\boldsymbol{\Psi}(x, t)=\int_{0}^{\infty} \mathrm{d} k \widetilde{\psi}(k) \mathrm{e}^{-\mathrm{i} \hbar k^{2} t / 2 m} \boldsymbol{\Phi}_{k}(x),
$$

where $\widetilde{\psi}(k)$ denotes the momentum amplitude that the initial wave packet would have at $t=0$ in absence of the laser.

With Eqs. (3.6) and (3.17), the probability for no photon detection up to time $t$ is given by

$$
N(t)=\|\Psi(t)\|^{2},
$$

and the probability density for the first photon reads

$$
w_{1}(t)=-\frac{\mathrm{d}}{\mathrm{d} t} N(t)=\frac{\mathrm{i}}{\hbar}\left\langle\boldsymbol{\Psi}(t)\left|H_{\mathrm{c}}-H_{\mathrm{c}}^{\dagger}\right| \Psi(t)\right\rangle .
$$

Since $H_{\mathrm{c}}-H_{\mathrm{c}}^{\dagger}=-\mathrm{i} \hbar \gamma|2\rangle\langle 2|$, one finally has

$$
w_{1}(t)=\gamma \int_{-\infty}^{\infty} \mathrm{d} x\left|\psi^{(2)}(x, t)\right|^{2}
$$

This is a quite intuitive result: The probability density for the first photon equals the decay rate times the probability to find the atom at time $t$ in the internal excited state. Since the temporal distribution of the first photon is assumed to be related to an operational arrival time distribution $\Pi(t), w_{1}(t)$ is a first "guess" to such an distribution:

$$
\begin{aligned}
\Pi(t) \equiv w_{1}(t) & =\gamma \int_{-\infty}^{\infty} \mathrm{d} x\left|\psi^{(2)}(x, t)\right|^{2} \\
& =\gamma \int_{0}^{\infty} \mathrm{d} k \int_{0}^{\infty} \mathrm{d} k^{\prime} \overline{\widetilde{\psi}(k)} \widetilde{\psi}\left(k^{\prime}\right) \mathrm{e}^{\mathrm{i} \hbar\left(k^{2}-k^{\prime 2}\right) t / 2 m} \int_{-\infty}^{\infty} \mathrm{d} x \overline{\phi_{k}^{(2)}(x)} \phi_{k^{\prime}}^{(2)}(x) .
\end{aligned}
$$

From Eq. (3.45) it is seen that this expression coincides with the trace of the reset density matrix, i.e. with the probability of a resetting event.

A typical example for such an operational arrival-time distribution is shown in Fig. 3.3, where $\Pi(t)$ is compared with the flux $J(t)$ and Kijowski's distribution $\Pi_{K}(t)$ for an initial Gaussian wave packet. It is seen that $\Pi(t)$ is either shifted with respect to the ideal distributions for a weak laser (delay) or it is not normalized for a strong laser (reflections). In the following section, the origin of these differences is investigated and methods are developed to eliminate them.

Throughout this work, Gaussian wave packets with a momentum distribution given by Eq. (2.19) are considered as examples. They are chosen in a way that numerically $|\widetilde{\psi}(k)|=0$ for $k \leq 0$. Therefore the under integral limit in Eq. (3.66) is justified. 


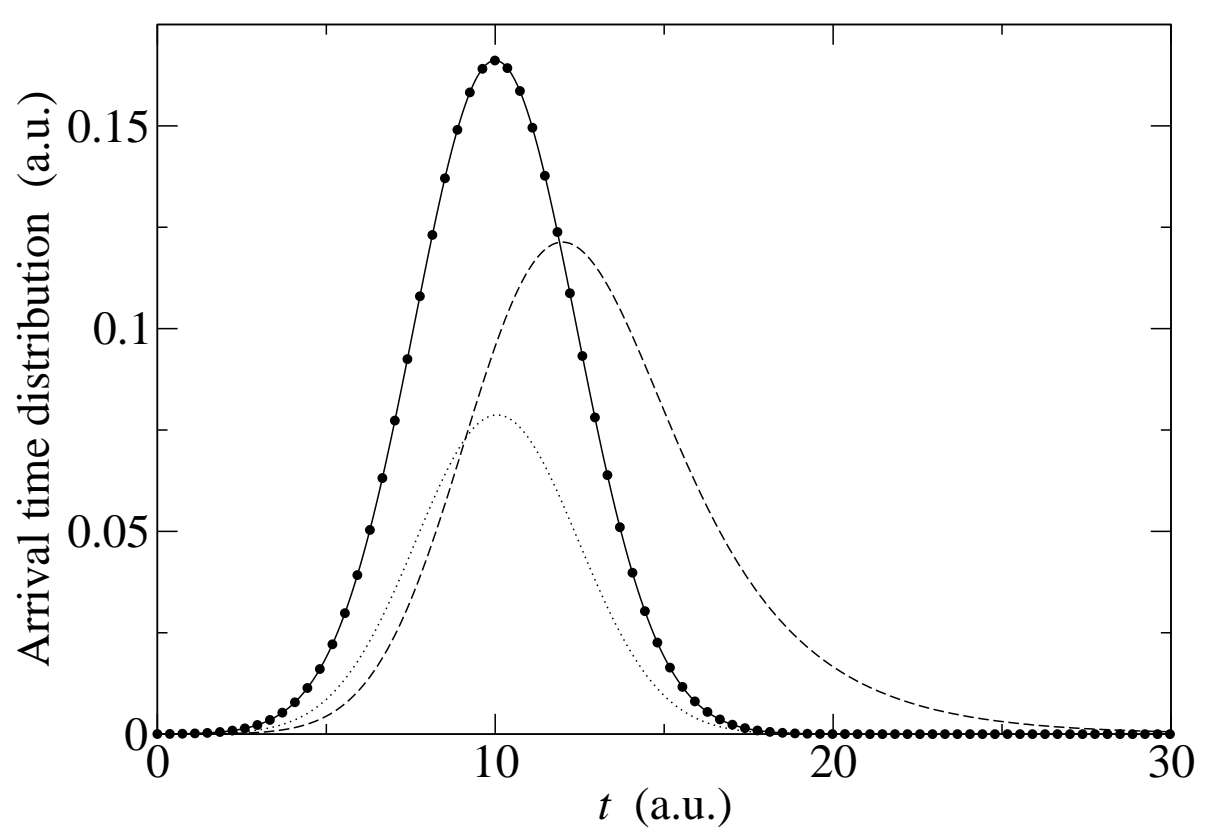

Figure 3.3: First-photon distribution $\Pi(t)$ for arrival at $x=0$ as an operational arrival-time distribution for $\Omega=0.1 \gamma_{\mathrm{Cs}}$ (dashed line) and $\Omega=10 \gamma_{\mathrm{Cs}}$ (dotted line), compared with Kijowski's distribution $\Pi_{K}(t)$ (circles) and the flux $J(t)$ (solid line). The initial state is a minimum-uncertainty Gaussian wave packet (see Eq. (2.19)) in the ground state with the center-of-mass motion of a cesium atom and with $\langle x\rangle=$ $-50.0, \Delta x=12.0,\langle v\rangle=5.0$. All figures in this work are for the resonant excitation of the transition $6^{2} P_{3 / 2}-6^{2} S_{1 / 2}$ of cesium with $\gamma_{\mathrm{Cs}}=33.3$ and in the atomic units defined in Appendix D.

Remark: Experimentally, it might be difficult to observe the very first photon emitted from the two-level atom due to a limited detector efficiency and the subsequent emission of many photons. With respect to the conditional time evolution, this problem can be solved by considering a three-level atom with an irreversible decay to a sink state $|3\rangle$ as shown in Fig. 3.1b. For this level type, it can be easily shown that the conditional Hamiltonian (3.55) remains the same, since it describes the time evolution before the first photon emission. In particular, the level $|3\rangle$ does not appear in the conditional time evolution. Thus, the first-photon distribution is identical with Eq. (3.70). The advantage with this kind of level scheme is that only first-photon events are recorded experimentally.

\subsubsection{Detection delay and reflection}

It has been shown [24] that two effects are responsible for the difference between the operational distribution $\Pi(t)$ and ideal ones, namely reflection and delay.

Reflection: The mathematical background for the occurrence of reflections is the step function in the potential, leading to eigenstates of the form of Eq. (3.57). The 
physical reason for that is not the recoil by the spontaneously emitted photon, since only the conditional time-development is considered, but rather the coupling to the laser field. The laser changes the internal occupation probabilities of the atom, thus its dipole moment; this in turn changes the electromagnetic field of the laser and hence its momentum distribution and because of momentum conservation the momentum distribution of the atom is changed ${ }^{1}$. It follows that in general not every atom can be observed and as a consequence the distribution $\Pi(t)$ is not normalized to 1, as shown in Fig. 3.3. With Eq. (3.68) one has for the integrated first-photon distribution,

$$
\int_{-\infty}^{\infty} \mathrm{d} t \Pi(t)=N(-\infty)-N(\infty)=1-N(\infty)
$$

In this equation, $N(\infty)$ is the probability that no photon is emitted at all. This probability can be derived by the fact that for large $t$ only the reflected ground-state part of Eq. (3.66) remains and all other terms are damped away,

$$
\Psi(x, t) \sim \int_{0}^{\infty} \mathrm{d} k \widetilde{\psi}(k) \mathrm{e}^{-\mathrm{i} \hbar k^{2} t / 2 m} R_{1}(k) \mathrm{e}^{-\mathrm{i} k x}|1\rangle, \quad t \rightarrow \infty .
$$

With Eq. (3.67) follows

$$
N(\infty)=\int_{0}^{\infty} \mathrm{d} k\left|R_{1}(k)\right|^{2}|\widetilde{\psi}(k)|^{2},
$$

i.e. the probability that the arrival of the atom is not detected equals the reflection probability of the ground state. This probability can be experimentally adjusted by the parameters $\Omega$ and $\gamma$. In the following and throughout this work, limits are always understood with respect to the smallest contribution of the kinetic energy to the considered wave packet, i.e. $\gamma \rightarrow \infty$ means $\hbar \gamma \gg \widetilde{E}_{k}$, etc. It is clear that the wave packet for $\Omega \rightarrow 0$ is not affected by the laser and evolves freely. This has been verified by applying the limit $\Omega \rightarrow 0$ to the expression for $R_{1}(k)$, leading to

$$
R_{1}(k) \sim \mathcal{O}\left(\Omega^{2}\right) \rightarrow 0, \quad \Omega \rightarrow 0 .
$$

On the other hand, also the limit $\gamma \rightarrow \infty$ yields [24]

$$
R_{1}(k) \rightarrow 0, \quad \gamma \rightarrow \infty,
$$

and leads consequently to a vanishing non-detection probability.

For later purpose, the limit of strong driving and strong decay is investigated, too, where $\gamma / \Omega$ is held fixed. In that case the reflection probability tends to 1 ,

$$
R_{1}(k) \rightarrow-1, \quad \gamma \rightarrow \infty, \gamma / \Omega=\text { const. }
$$

Additionally, one concludes from Eq. (3.73) that reflection is a problem only for slow atoms, since for wave packets peaked sharply around a $k_{0}$ with $R_{1}\left(k_{0}\right) \approx 1$ one has $N(\infty) \approx 1$, whereas for fast atoms the reflection probability tends to zero.

\footnotetext{
${ }^{1} \mathrm{~A}$ similar effect arises in classical mechanics for a moving dipole, impinging on a homogeneous electric field in the right half-space. From the Hamiltonian equations of motion one sees that the $x$-dependence in the electric field leads to a change of the center-of-mass momentum of the dipole.
} 
Delay: The second effect that causes a difference between $\Pi(t)$ and ideal distributions is the delay in the photon emission due to the excitation time and the lifetime of the upper level. For a two-level atom in the ground state at rest, the waiting time distribution for the first photon is given by [96]

$$
W(t)=\frac{\gamma \Omega^{2}}{4|S|^{2}} \mathrm{e}^{-\gamma t / 2}\left|\mathrm{e}^{S t / 2}-\mathrm{e}^{-S t / 2}\right|^{2}
$$

with $S=\frac{1}{2} \sqrt{\gamma^{2}-4 \Omega^{2}}$. For the mean waiting time follows

$$
t_{W}=\int_{0}^{\infty} \mathrm{d} t W(t) t=\frac{\gamma^{2}+2 \Omega^{2}}{\gamma \Omega^{2}} .
$$

By means of this expression, there are seen useful limits to overcome the delay problem. For weak driving or strong decay one has negligible reflection, but large delay,

$$
t_{W} \sim \gamma / \Omega^{2} \rightarrow \infty, \quad \Omega \rightarrow 0 \text { or } \gamma \rightarrow \infty
$$

The delay also becomes large for strong driving, $\Omega \rightarrow \infty$, and negligible decay, $\gamma \rightarrow 0$

$$
t_{W} \sim 2 / \gamma \rightarrow \infty, \quad \Omega \rightarrow \infty, \gamma \rightarrow 0
$$

The delay may be avoided by strong driving and strong decay, where the ratio of $\Omega$ and $\gamma$ is held fixed, leading to

$$
t_{W} \sim 1 / \gamma \rightarrow 0, \quad \Omega \rightarrow \infty, \Omega / \gamma=\text { const. }
$$

With Eqs. (3.74)-(3.76) and Eqs. (3.79)-(3.81) it is obvious, that the conditions to overcome the reflection and the delay are conflicting. Nevertheless, for wave packets centered around some $p_{0}$ in momentum space, there are parameter regimes with negligible delay and reflection [24]. If $(\Delta t)_{\Pi}$ denotes the width of $\Pi(t)$ and $\widetilde{E}$ denotes the smallest significant energy component of the wave packet, the following two regimes lead to negligible reflection and delay with respect to ideal distributions:

$$
\begin{gathered}
\frac{\hbar}{\widetilde{E}} \lesssim \frac{\gamma}{\Omega^{2}} \ll(\Delta t)_{\Pi} \quad \text { (weak driving regime) } \\
\frac{\hbar}{\widetilde{E}} \ll \frac{1}{\Omega} \ll \frac{2}{\gamma} \ll(\Delta t)_{\Pi} \quad \text { (strong driving regime) }
\end{gathered}
$$

\subsubsection{Deconvolution and ideal distributions}

A method to overcome the delay problem has already pointed out by Allcock [6] in connection with imaginary potentials and it has recently been used for the laser model under consideration [24]. For later purpose, the derivation presented in Ref. [24] is explained in the following in detail.

The idea is that an ideal arrival time distribution $\Pi_{i d}(t)$ might be hidden in $\Pi(t)$, but it is convoluted with the delay time distribution $W(t)$ of Eq. (3.77). The ansatz

$$
\Pi(t)=\left(\Pi_{\mathrm{id}} * W\right)(t)
$$


where $*$ denotes the convolution, can be solved for $\Pi_{\mathrm{id}}$ by a Fourier transformation,

$$
\widetilde{\Pi}_{\mathrm{id}}(\nu)=\frac{\widetilde{\Pi}(\nu)}{\widetilde{W}(\nu)} .
$$

With Eqs. (3.70) and (3.77) one has

$$
\begin{aligned}
\widetilde{\Pi}(\nu) & =\int_{-\infty}^{\infty} \mathrm{d} t \mathrm{e}^{-\mathrm{i} \nu t} \Pi(t) \\
& =\gamma \int_{0}^{\infty} \mathrm{d} k \int_{0}^{\infty} \mathrm{d} k^{\prime} \bar{\psi}(k) \widetilde{\psi}\left(k^{\prime}\right) 2 \pi \delta\left(\nu-\hbar\left(k^{2}-k^{\prime 2}\right) / 2 m\right) \int_{-\infty}^{\infty} \mathrm{d} x \overline{\phi_{k}^{(2)}(x)} \phi_{k^{\prime}}^{(2)}(x)
\end{aligned}
$$

and

$$
\frac{1}{\widetilde{W}(\nu)}=1+\left(\frac{\gamma}{\Omega^{2}}+\frac{2}{\gamma}\right)(\mathrm{i} \nu)+\frac{3}{\Omega^{2}}(\mathrm{i} \nu)^{2}+\frac{2}{\gamma \Omega^{2}}(\mathrm{i} \nu)^{3} .
$$

Since multiplication with $\mathrm{i} \nu$ in the Fourier space corresponds to a derivative in time, the transformation of Eq. (3.85) back in the time domain yields

$$
\Pi_{\mathrm{id}}(t)=\Pi(t)+\left(\frac{\gamma}{\Omega^{2}}+\frac{2}{\gamma}\right) \Pi^{\prime}(t)+\frac{3}{\Omega^{2}} \Pi^{\prime \prime}(t)+\frac{2}{\gamma \Omega^{2}} \Pi^{\prime \prime \prime}(t) .
$$

Assuming that the delay problem is solved by this construction, one clearly attempts to simultaneously minimize reflections. This can be done by considering the limit $\gamma \rightarrow \infty$ of Eq. (3.75). In that case, one has in leading order

$$
\begin{aligned}
\frac{1}{\widetilde{W}(\nu)} & \sim \frac{\mathrm{i} \nu \gamma}{\Omega^{2}}, \\
\lambda_{+} & \sim-\frac{\mathrm{i} \Omega^{2}}{2 \gamma} \rightarrow 0, \\
\lambda_{-} & \sim-\frac{\mathrm{i} \gamma}{2} \rightarrow \infty, \\
k_{+} & \sim k+\frac{\mathrm{i} m \Omega^{2}}{2 \hbar k \gamma} \rightarrow k, \\
k_{-} & \sim q \sim \sqrt{\frac{\mathrm{i} m \gamma}{\hbar}}, \\
C_{-}, R_{1}, R_{2} & \rightarrow 0, \\
C_{+} & \rightarrow 1,
\end{aligned}
$$

Thus, $\gamma \overline{\phi_{k}^{(2)}(x)} \phi_{k^{\prime}}^{(2)}(x)$ is exponentially damped for $x \leq 0$, whereas for $x \geq 0$ only the terms with $k_{+}$survive, leading to

$$
\gamma \overline{\phi_{k}^{(2)}(x)} \phi_{k^{\prime}}^{(2)}(x) \sim \frac{\Omega^{2}}{2 \pi \gamma} \mathrm{e}^{-\mathrm{i}\left(\bar{k}_{+}-k_{+}^{\prime}\right) x} \Theta(x), \quad \gamma \rightarrow \infty .
$$


Inserting this into Eq. (3.86), integrating over $x$ and inserting the result together with Eq. (3.87) into Eq. (3.85) yields

$$
\widetilde{\Pi}_{\mathrm{id}}(\nu) \rightarrow \int_{0}^{\infty} \mathrm{d} k \int_{0}^{\infty} \mathrm{d} k^{\prime} \overline{\widetilde{\psi}(k)} \widetilde{\psi}\left(k^{\prime}\right) \delta\left(\nu-\hbar\left(k^{2}-k^{\prime 2}\right) / 2 m\right) \frac{\nu}{k-k^{\prime}} .
$$

In the time domain one finally has

$$
\begin{aligned}
\Pi_{\mathrm{id}}(t) & =\frac{1}{2 \pi} \int_{-\infty}^{\infty} \mathrm{d} t \mathrm{e}^{\mathrm{i} \nu t} \widetilde{\Pi}_{\mathrm{id}}(\nu) \\
& \rightarrow \frac{\hbar}{2 \pi m} \int_{0}^{\infty} \mathrm{d} k \int_{0}^{\infty} \mathrm{d} k^{\prime} \overline{\widetilde{\psi}(k)} \widetilde{\psi}\left(k^{\prime}\right) \mathrm{e}^{\mathrm{i} \hbar\left(k^{2}-k^{\prime 2}\right) / 2 m} \frac{k+k^{\prime}}{2} \\
& =J(t, x=0), \quad \text { for } \gamma \rightarrow \infty
\end{aligned}
$$

This remarkable result of Ref. [24] relates the operational arrival-time distribution to the quantum mechanical flux for the free wave function $\psi(x, t)$ at $x=0$. Thus, the first-photon detection provides an approach to measuring the flux, if a deconvolution is performed afterwards on the experimental data. This is in contrast to the suggestions made in some textbooks [97] concerning the measurability of the flux.

An example of this procedure is shown in Fig. 3.4. Here the difference between the flux and Kijowski's distribution is enhanced by considering a coherent superposition of two Gaussian wave packets that will have a minimal position-momentum uncertainty at $x=0$ in the absence of the laser, respectively. The superposition is chosen in a way that the backflow effect occurs, i.e. $J(t)<0$ for some $t$ (see Section 2.1.3). One clearly recognizes the delay in $\Pi(t)$ which is eliminated in the deconvoluted distribution $\Pi_{\mathrm{id}}(t)$ that is right on top of the flux $J$.

To conclude, it is possible to eliminate the decay and the reflection problem simultaneously by performing a deconvolution in the limit of large decay rates or weak driving. This approach does not conserve the positivity of the distribution, and one ends up with a not necessarily positive quasi-distribution, the quantum mechanical flux.

\subsubsection{Normalized arrival-time distributions}

In the previous section it has been shown that the procedure of deconvolution corrects the operational distribution of Eq. (3.70) for the delay, and together with a choice of parameters that minimize the reflection probability (strong decay or weak driving) an ideal distribution is obtained.

One may ask, whether such a proceeding can be applied the other way around: To choose the parameters to minimize the delay (see Eq. (3.81)) and to use a procedure that gets rid off the reflection problem. A simple solution for this problem is given by a normalization of the first-photon distribution, since this eliminates the consequences of the reflection. A natural way to normalize $\Pi(t)$ is the division of $\Pi(t)$ by its integral, i.e. defining a new distribution

$$
\Pi_{\mathrm{N}}(t)=\frac{\Pi(t)}{\int_{-\infty}^{\infty} \mathrm{d} t \Pi(t)} .
$$




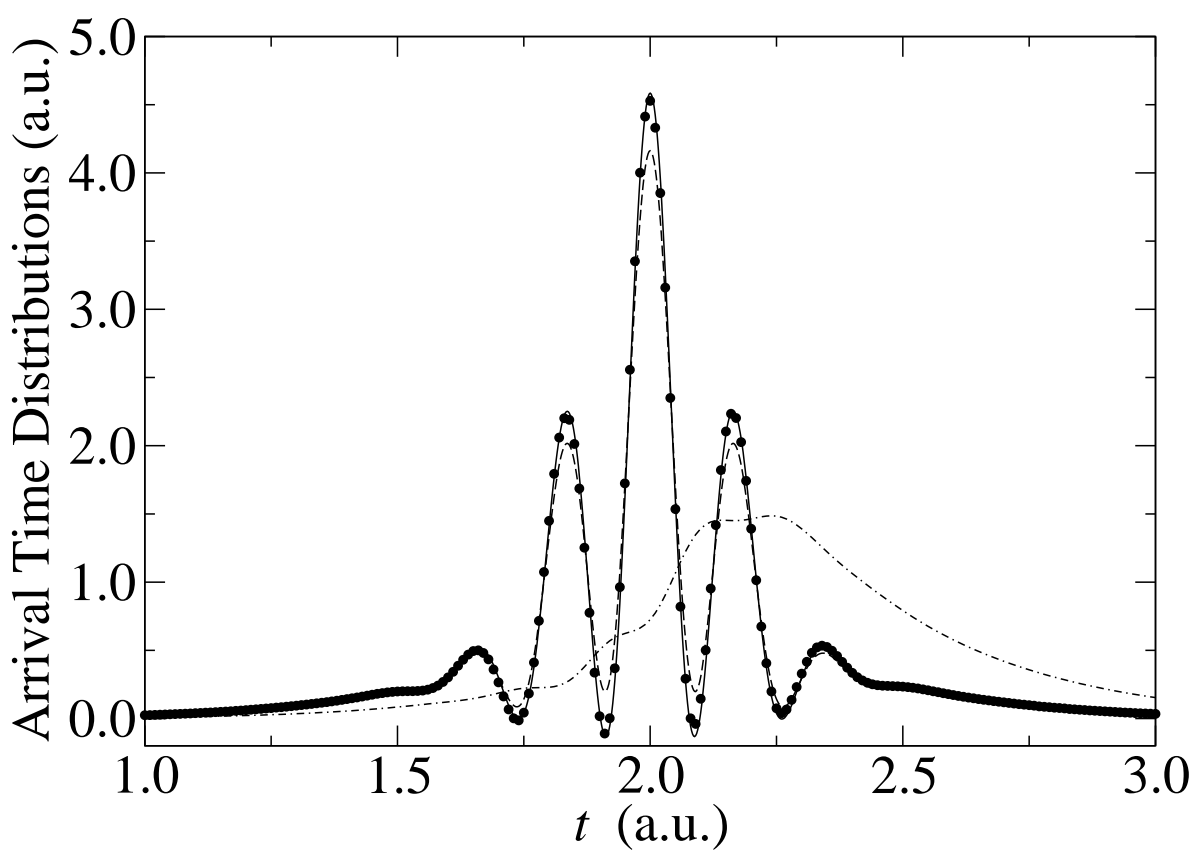

Figure 3.4: Comparison between the ideal arrival-time distributions $J(t)$ (solid line) and $\Pi_{\mathrm{K}}(t)$ (dashed line) and the operational first-photon distributions $\Pi(t)$ (dasheddotted line) and the deconvoluted $\Pi_{\mathrm{id}}(t)$ (circles) for $\Omega=0.37 \gamma_{\mathrm{Cs}}$. The initial wave packet is a coherent superposition $\psi=\left(\psi_{1}+\psi_{2}\right) / \sqrt{2}$ of two Gaussian states for the center-of-mass motion of a single cesium atom, such that they become minimumuncertainty packets at $x=0$ and $t=2$ with $\Delta x_{1}=\Delta x_{2}=1.0,\langle v\rangle_{1}=2.54$, $\langle v\rangle_{2}=8.90$.

This can be effective in some parameter regimes, when the delay is small. An example is shown in Fig. 3.5, where $\Pi_{\mathrm{N}}(t)$ perfectly agrees with the flux $J(t)$, but not with Kijowski's distribution. It is important to note that $\Pi_{N}(t)$ is necessarily positive, in contrast to $\Pi_{\text {id }}(t)$.

An ideal distribution is obtained by applying the limit $\gamma \rightarrow \infty, \gamma / \Omega=$ const. to $\Pi_{\mathrm{N}}(t)$, since this eliminates the delay time according to Eq. (3.81). By setting

$$
\alpha \equiv \sqrt{1-\frac{4 \Omega^{2}}{\gamma^{2}}}=\text { const. }
$$

one has in leading order

$$
\begin{aligned}
\lambda_{ \pm} & =\frac{\mathrm{i} \gamma}{4}(-1 \pm \alpha), \\
q & \sim \sqrt{\frac{\mathrm{i} m \gamma}{\hbar}}, \\
k_{ \pm} & \sim q \sqrt{\frac{1 \mp \alpha}{2}}, \\
R_{1} & \sim-1-\frac{2 \mathrm{i} k}{\gamma^{1 / 2}} \sqrt{\frac{\mathrm{i} \hbar}{m}} c_{1}(\alpha),
\end{aligned}
$$




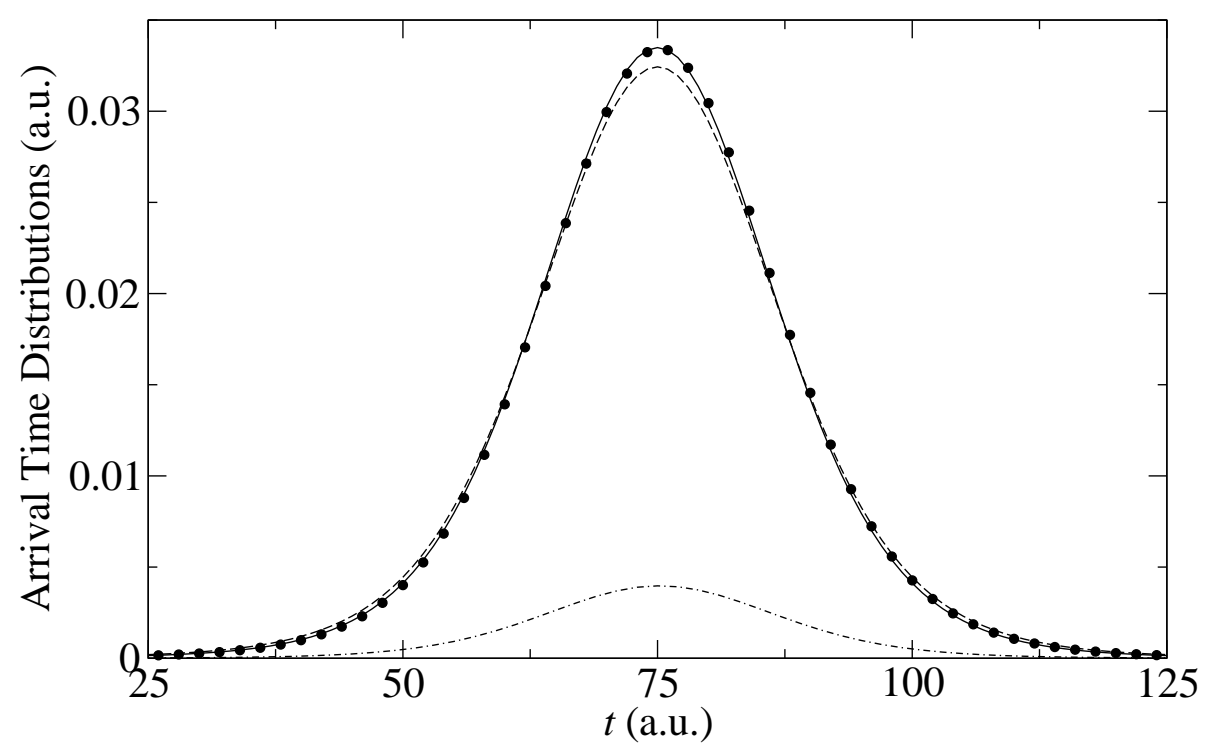

Figure 3.5: Normalized first-photon distribution for suitable parameter regime: $\Pi_{\mathrm{N}}(t)$ (circles) agrees perfectly with the flux $J(t)$ (solid line), but it is different from Kijowski's distribution $\Pi_{\mathrm{K}}(t)$ (dashed line). $\Pi(t)$ (dot-dashed line) is not normalized, since $\Omega=3 \gamma_{\mathrm{Cs}}$ is large with respect to $E / \hbar=m\langle v\rangle^{2} / 2 \hbar ;\langle v\rangle=0.42, \Delta x=5.0$. The initial Gaussian wave packet is chosen to become a minimum-uncertainty state when its center arrives at $x=0$ and $t=75$.

$$
R_{2} \sim-\frac{k}{\gamma^{1 / 2}} \sqrt{\frac{\mathrm{i} \hbar}{m}} c_{2}(\alpha)
$$

where the constants $c_{i}(\alpha)$ have been defined for convenience by

$$
\begin{aligned}
c_{1}(\alpha) & =\frac{2 \sqrt{2} \alpha+(1+\alpha)^{3 / 2}-(1-\alpha)^{3 / 2}}{\sqrt{2} \alpha \sqrt{1-\alpha^{2}}+\sqrt{\alpha+1}(\alpha-1)+\sqrt{1-\alpha}(1+\alpha)}, \\
c_{2}(\alpha) & =\frac{2 \sqrt{2} \sqrt{1-\alpha^{2}}(\sqrt{1+\alpha}-\sqrt{1-\alpha})}{\sqrt{1+\alpha}(\sqrt{2}+\sqrt{1-\alpha})(\alpha-1)+\sqrt{1-\alpha}(\sqrt{2}+\sqrt{1+\alpha})(\alpha+1)},(3.1 \\
c_{3}(\alpha) & =\frac{1}{2}[\sqrt{1+\alpha}(\sqrt{2}+\sqrt{1-\alpha})(\alpha-1)+\sqrt{1-\alpha}(\sqrt{2}+\sqrt{1+\alpha})(\alpha+1)] .
\end{aligned}
$$

Their particular form turns out to be irrelevant in the following. With Eq. (3.57) it follows that

$$
\begin{aligned}
\gamma \overline{\phi_{k}^{(2)}(x)} \phi_{k^{\prime}}^{(2)}(x) & \sim \frac{\hbar k k^{\prime}}{2 \pi m}\left\{\Theta(-x) c_{2}^{2}(\alpha) \mathrm{e}^{-\mathrm{i}(q-\bar{q}) x}\right. \\
& \left.+\Theta(x) \frac{16}{c_{3}^{2}(\alpha)} \frac{\Omega^{2}}{\gamma^{2}}\left|\left(1+\sqrt{\frac{1+\alpha}{2}}\right) \mathrm{e}^{\mathrm{i} k_{+} x}-\left(1+\sqrt{\frac{1-\alpha}{2}}\right) \mathrm{e}^{\mathrm{i} k-x}\right|^{2}\right\}
\end{aligned}
$$

The term in brackets is independent of $k$, therefore the integral over $x$ is canceled by the normalization. With Eq. (3.93) one obtains for the normalized first-photon 
distribution the following result:

$$
\begin{aligned}
\Pi_{\mathrm{N}}(t) & \rightarrow \frac{\int_{0}^{\infty} \mathrm{d} k \mathrm{~d} k^{\prime} \bar{\psi}(k) \widetilde{\psi}\left(k^{\prime}\right) \mathrm{e}^{\mathrm{i} \hbar\left(k^{2}-k^{\prime 2}\right) t / 2 m} k k^{\prime}}{\int_{-\infty}^{\infty} \mathrm{d} t \int_{0}^{\infty} \mathrm{d} k \mathrm{~d} k^{\prime} \overline{\widetilde{\psi}(k)} \widetilde{\psi}\left(k^{\prime}\right) \mathrm{e}^{\mathrm{i} \hbar\left(k^{2}-k^{\prime 2}\right) t / 2 m} k k^{\prime}} \\
& =\frac{\hbar}{2 \pi m\langle k\rangle_{\psi}} \int_{0}^{\infty} \mathrm{d} k \mathrm{~d} k^{\prime} \overline{\widetilde{\psi}(k)} \widetilde{\psi}\left(k^{\prime}\right) \mathrm{e}^{\mathrm{i} \hbar\left(k^{2}-k^{\prime 2}\right) t / 2 m} k k^{\prime} \\
& =\frac{\hbar}{m\langle k\rangle_{\psi}}\left\langle\psi_{\mathrm{f}}(t)|\widehat{k} \delta(\widehat{x}) \widehat{k}| \psi_{\mathrm{f}}(t)\right\rangle, \quad \gamma \rightarrow \infty, \gamma / \Omega=\text { const. }
\end{aligned}
$$

Eq. (3.104) describes again an ideal distribution, evaluated at $x=0$ in terms of the free wave function

$$
\left|\psi_{\mathrm{f}}(t)\right\rangle=\int \mathrm{d} k \widetilde{\psi}(k) \mathrm{e}^{-\mathrm{i} \hbar k^{2} t / 2 m}|k\rangle
$$

and normalized by its mean momentum $\langle k\rangle_{\psi}=\int \mathrm{d} k k|\widetilde{\psi}(k)|^{2}$. It is fundamentally different from the flux and from Kijowski's distribution and can be considered as a third ideal arrival time distribution. It is shown later in Section 3.5 that the derived expression for $\Pi_{\mathrm{N}}(t)$ is related to a local kinetic energy density at $x=0$, and the above approach gives an operational procedure to measure such a local energy density.

To conclude, a second method to simultaneously eliminate the decay and the reflection problem is given by a normalization in the limit of vanishing delay, i.e. $\gamma \rightarrow$ $\infty$ with $\gamma / \Omega=$ const. This yields a positive, ideal distribution which is different from $J(t)$ and $\Pi_{K}(t)$. The main difference is due to the fact that $\Pi_{N}(t)$ is not a bilinear form of the state $\psi_{t}$, since the normalization destroys this property. Therefore, in Section 5.1, a new normalization procedure is developed which conserves the property of a bilinear form.

\subsubsection{Galilean invariance of the first-photon distribution}

One may ask if instead of considering a moving atom, impinging on a spatially localized laser field, one could consider an atom rest and a moving laser field. Both situations are connected by a Galilei transformation. It can indeed be shown that the result for the first-photon distribution is the same for both cases [98].

First, one writes the conditional Hamiltonian (3.55) in the form

$$
H_{\mathrm{c}}=\frac{\widehat{p}^{2}}{2 m}+V(\widehat{x})
$$

and denotes the corresponding time evolution operator by

$$
U_{\mathrm{c}}(t, 0)=\mathrm{e}^{-\mathrm{i} H_{\mathrm{c}} t / \hbar}
$$

Next, a new time evolution operator $U_{\mathrm{c}}^{v}(t, 0)$ is defined by

$$
U_{\mathrm{c}}^{v}(t, 0)=\mathrm{e}^{-\mathrm{i} m v \widehat{x} / \hbar} \mathrm{e}^{\mathrm{i} v t \widehat{p} / \hbar} U_{\mathrm{c}}(t, 0) \mathrm{e}^{\mathrm{i} m v \widehat{x} / \hbar} .
$$


Using the relations

$$
\begin{aligned}
\mathrm{e}^{\mathrm{i} a \widehat{p} / \hbar} f(\widehat{x}) \mathrm{e}^{-\mathrm{i} a \widehat{p} / \hbar} & =f(\widehat{x}+a), \\
\mathrm{e}^{-\mathrm{i} b \widehat{x} / \hbar} g(\widehat{p}) \mathrm{e}^{\mathrm{i} b \widehat{x} / \hbar} & =g(\widehat{p}+b)
\end{aligned}
$$

for some constants $a, b$, one has the following result for the time derivative of $U_{\mathrm{c}}^{v}(t, 0)$ :

$$
\begin{aligned}
\mathrm{i} \hbar \frac{\partial}{\partial t} U_{\mathrm{c}}^{v}(t, 0) & =\mathrm{i} \hbar \frac{\partial}{\partial t}\left(\mathrm{e}^{-\mathrm{i} m v \widehat{x} / \hbar} \mathrm{e}^{\mathrm{i} v t \widehat{p} / \hbar} \mathrm{e}^{-\mathrm{i} H_{\mathrm{c}} t / \hbar} \mathrm{e}^{\mathrm{i} m v \widehat{x} / \hbar}\right) \\
& =\mathrm{i} \hbar\left(\mathrm{e}^{-\mathrm{i} m v \widehat{x} / \hbar} \mathrm{e}^{\mathrm{i} v t \widehat{p} / \hbar}\left(\mathrm{i} v \widehat{p} / \hbar-\mathrm{i} H_{\mathrm{c}} / \hbar\right) \mathrm{e}^{-\mathrm{i} H_{\mathrm{c}} t / \hbar} \mathrm{e}^{\mathrm{i} m v \widehat{x} / \hbar}\right) \\
& =\mathrm{e}^{-\mathrm{i} m v \widehat{x} / \hbar} \mathrm{e}^{\mathrm{i} v t \widehat{p} / \hbar}\left(\frac{\widehat{p}^{2}}{2 m}-v \widehat{p}+V(\widehat{x})\right) \mathrm{e}^{-\mathrm{i} v t \widehat{p} / \hbar} \mathrm{e}^{\mathrm{i} m v \widehat{x} / \hbar} \mathrm{e}^{-\mathrm{i} m v \widehat{x} / \hbar} U_{\mathrm{c}}^{v}(t, 0) \\
& =\left(\frac{(\widehat{p}+m v)^{2}}{2 m}-v(\widehat{p}+m v)+V(\widehat{x}+v t)\right) U_{\mathrm{c}}^{v}(t, 0) \\
& =\left(\frac{\widehat{p}^{2}}{2 m}+V(\widehat{x}+v t)-\frac{1}{2} m v^{2}\right) U_{\mathrm{c}}^{v}(t, 0) .
\end{aligned}
$$

This shows that $U_{\mathrm{c}}^{v}(t, 0)$ is the time development operator for the Hamiltonian

$$
H_{\mathrm{c}}^{v}=\frac{\widehat{p}^{2}}{2 m}+V(\widehat{x}+v t)-\frac{1}{2} m v^{2} .
$$

Up to the constant term $\frac{1}{2} m v^{2}$ which leads to a global phase, this Hamiltonian describes a potential which moves with velocity $v$. Applying $U_{\mathrm{c}}^{v}(t, 0)$ to a twocomponent wave function shifted in momentum by $-m v$ to compensate the centerof-mass motion of the atom,

$$
U_{\mathrm{c}}^{v}(t, 0) \mathrm{e}^{-\mathrm{i} m v \widehat{x} / \hbar}|\Psi\rangle,
$$

one concludes that $\left\|\psi_{t}^{(2)}\right\|^{2}$, which enters into Eq. (3.70), is not changed and the result for the first-photon distribution remains the same.

\subsection{Arrival-time measurement with narrow laser fields}

\subsubsection{The probability density for the first photon}

The previous modeling of arrival times by means of the fluorescence of atoms, impinging on a half-space laser field, was well suited for situations where the particles come in solely from one side. Of course, this requirement is in general too constricting. An interesting and widely discussed problem for arrival-time distributions is the question of an antisymmetric superposition of two wave packets, coming in to $x=0$ from opposite sides $[39,40]$ such that $|\psi(x, t)|^{2}$ vanishes at $x=0$. It is shown later in Section 6.2 that the operational approach can give an answer to that question, 
if the laser field is considered to be very narrow (Fig. 3.6). In the following, one assumes the laser shape to be

$$
\Omega(x)=\frac{L_{0} \Omega}{2 \epsilon} \chi_{[-\epsilon, \epsilon]}(x),
$$

where the characteristic function $\chi_{[a, b]}(x)$ is one for $a \leq x \leq b$ and zero elsewhere, and where $2 \epsilon$ is the width of the region and $L_{0} / \epsilon$ is a number that determines the scaling of the Rabi frequency. For $\epsilon$ much smaller than the width of the wave packet, and large Rabi frequencies $\Omega(x) \sim 1 / \epsilon$, one can assume the limit $\epsilon \rightarrow 0$ to be a good approximation, yielding

$$
\Omega(x)=\Omega L_{0} \delta(x)
$$

Moving atom = wave packet

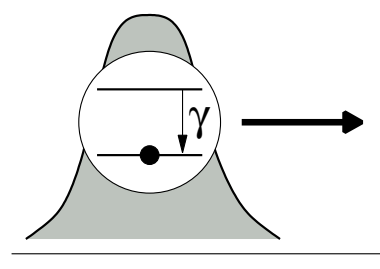

Narrow laser field, Rabi frequency $\Omega \sim 1 / \varepsilon$

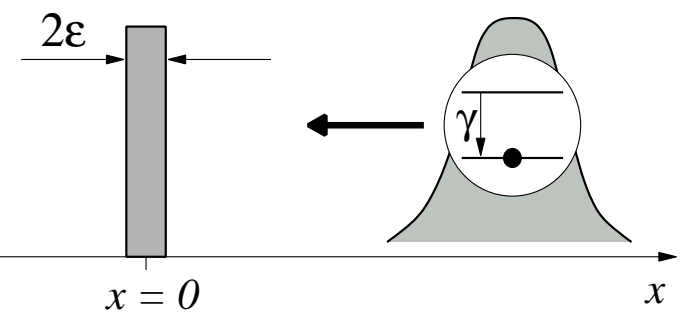

Figure 3.6: Scheme of arrival-time measurement with a narrow laser field. In principle, this allows the investigation of arrival-times for a coherent superposition of two packets, coming in from opposite sides as discussed in Refs. [39, 40].

The conditional Hamiltonian of this model is given by

$$
H_{\mathrm{c}}=\frac{\widehat{p}^{2}}{2 m}+\frac{\hbar}{2}\left(\begin{array}{cc}
0 & \Omega L_{0} \delta(\widehat{x}) \\
\Omega L_{0} \delta(\widehat{x}) & -\mathrm{i} \gamma
\end{array}\right)
$$

Solving the stationary Schrödinger equation

$$
H_{\mathrm{c}} \boldsymbol{\Phi}_{k}=E_{k} \boldsymbol{\Phi}_{k}
$$

for a left incoming wave by means of the transfer matrix method given in Appendix B.2, one obtains for the energy eigenstates

$$
\boldsymbol{\Phi}_{k}(x)=\frac{1}{\sqrt{2 \pi}} \begin{cases}\left(\begin{array}{c}
\mathrm{e}^{\mathrm{i} k x}+R_{1} \mathrm{e}^{-\mathrm{i} k x} \\
R_{2} \mathrm{e}^{-\mathrm{i} q x}
\end{array}\right), & x \leq 0 \\
\left(\begin{array}{l}
T_{1} \mathrm{e}^{\mathrm{i} k x} \\
T_{2} \mathrm{e}^{\mathrm{i} q x}
\end{array}\right), & x \geq 0\end{cases}
$$

with

$$
q=\sqrt{k^{2}+\mathrm{i} m \gamma / \hbar}, \quad \operatorname{Im} q>0 .
$$


The reflection and transmission coefficients are given by

$$
\begin{aligned}
R_{1} & =-\frac{m^{2} \Omega^{2} L_{0}^{2}}{4 \hbar^{2} k q+m^{2} \Omega^{2} L_{0}^{2}}, \\
R_{2}=T_{2} & =\frac{-2 \mathrm{i} \hbar m k \Omega L_{0}}{4 \hbar^{2} k q+m^{2} \Omega^{2} L_{0}^{2}}, \\
T_{1} & =\frac{4 \hbar^{2} k q}{4 \hbar^{2} k q+m^{2} \Omega^{2} L_{0}^{2}} .
\end{aligned}
$$

A wave packet $\Psi(x, t)$, coming in from the left, is given as before by Eq. (3.66), and the probability for the observation of the first photon at $t$ is given by Eq. (3.70). The difference here is the probability of no emission at all. Since the wave packet for large $t$ contains contributions from the reflected and from the transmitted part, it is asymptotically given by

$$
\boldsymbol{\Psi}(x, t) \sim \int_{0}^{\infty} \mathrm{d} k \widetilde{\psi}(k) \mathrm{e}^{-\mathrm{i} \hbar k^{2} t / 2 m}\left(R_{1}(k) \mathrm{e}^{-\mathrm{i} k x} \Theta(-x)+T_{1}(k) \mathrm{e}^{\mathrm{i} k x} \Theta(x)\right)|1\rangle, \quad t \rightarrow \infty .
$$

With Eq. (3.67) it follows that

$$
N(\infty)=\int_{0}^{\infty} \mathrm{d} k\left(\left|R_{1}(k)\right|^{2}+\left|T_{1}(k)\right|^{2}\right)|\widetilde{\psi}(k)|^{2} .
$$

This expression never vanishes, since either the reflection or the transmission probability of the ground state is larger than zero.

The unnormalized first-photon distribution for narrow laser fields can be derived in the same manner as it has been shown for the half-space laser field in Section 3.2.1. In analogy to Eq. (3.70), it takes the form

$$
\Pi_{\delta}(t)=\gamma \int_{0}^{\infty} \mathrm{d} k \mathrm{~d} k^{\prime} \bar{\psi}(k) \widetilde{\psi}\left(k^{\prime}\right) \mathrm{e}^{\mathrm{i} \hbar\left(k^{2}-k^{\prime 2}\right) t / 2 m} \int_{-\infty}^{\infty} \mathrm{d} x \overline{\phi_{k}^{(2)}(x)} \phi_{k^{\prime}}^{(2)}(x),
$$

where $\phi_{k}^{(2)}(x)$ is given here by the second component of Eq. (3.118).

\subsubsection{Parameter regimes for the narrow laser model}

For the delta-laser potential as well one can vary the parameters $\gamma$ and $\Omega$ to obtain ideal distributions in some limits. Since the atom is moving in the laser-free region for all $x$ except for the origin, the delay in the temporal distribution of the first photon is related to the waiting time distribution $W_{0}(t)$ of an excited atom at rest in the presence of decay, but without laser interaction. Note that this expression cannot be obtained by taking the limit $\Omega \rightarrow 0$ in Eq. (3.77), but instead it is given by

$$
W_{0}(t)=\gamma \mathrm{e}^{-\gamma t} .
$$

For the mean waiting time it follows that

$$
t_{W_{0}}=\int_{0}^{\infty} \mathrm{d} t t W_{0}(t)=\gamma^{-1} .
$$


This delay can be eliminated from the temporal first-photon distribution of the moving atom as shown in Section 3.2.3 by means of a deconvolution. The derivation is based on the inverse Fourier transform of $W_{0}(t)$,

$$
\frac{1}{\widetilde{W}_{0}(\nu)}=\frac{\gamma+\mathrm{i} \nu}{\gamma}
$$

In the following, $\Pi_{\delta}(t)$ of Eq. (3.125) is given for various parameter regimes of $\gamma$ and $\Omega$. For $\gamma \rightarrow 0$ one has a large waiting time $t_{W_{0}}$ and the deconvolution is applied.

Strong laser field $(\Omega \rightarrow \infty)$ : Here $\Pi_{\delta}(t)$ takes in leading order the form

$$
\Pi_{\delta}(t) \sim \frac{1}{2 \pi} \frac{8 \hbar^{2} \gamma}{m^{2} L_{0}^{2} \Omega^{2}} \int_{0}^{\infty} \mathrm{d} k \mathrm{~d} k^{\prime} \bar{\psi}(k) \widetilde{\psi}\left(k^{\prime}\right) \mathrm{e}^{\mathrm{i} \hbar\left(k^{2}-k^{\prime 2}\right) t / 2 m} \frac{k k^{\prime}}{i\left(\bar{q}-q^{\prime}\right)}, \quad \Omega \rightarrow \infty .
$$

For small decay rates, $\gamma \rightarrow 0$, the mean waiting time $t_{W_{0}}$ is large and one can try to eliminate the decay with a deconvolution as in Section 3.2.3. With Eq. (3.128) it follows that

$$
\frac{1}{\widetilde{W}_{0}(\nu)} \sim \frac{\mathrm{i} \nu}{\gamma}, \quad \gamma \rightarrow 0
$$

and this leads to a deconvoluted distribution

$\Pi_{\delta, \text { id }}(t) \sim \frac{2 \hbar^{3}}{\pi m^{3} L_{0}^{2} \Omega^{2}} \int_{0}^{\infty} \mathrm{d} k \mathrm{~d} k^{\prime} \widetilde{\psi}(k) \widetilde{\psi}\left(k^{\prime}\right) \mathrm{e}^{\mathrm{i} \hbar\left(k^{2}-k^{\prime 2}\right) t / 2 m} k k^{\prime}\left(k+k^{\prime}\right), \quad \Omega \rightarrow \infty, \gamma \rightarrow 0$.

It is not normalized, since in the limit $\Omega \rightarrow \infty$ reflections are enhanced. Normalizing $\Pi_{\delta \text {,id }}(t)$ does not yield an expression that is related to one of the ideal arrival-time distributions.

For large decay rates, $\gamma \rightarrow \infty$, one has $q \sim \sqrt{\mathrm{i} m \gamma / \hbar}$, and Eq. (3.129) becomes

$$
\Pi_{\delta}(t) \sim \frac{1}{2 \pi} \sqrt{\frac{\hbar}{2 m}} \frac{8 \hbar^{2} \sqrt{\gamma}}{m^{2} L_{0}^{2} \Omega^{2}} \int_{0}^{\infty} \mathrm{d} k \mathrm{~d} k^{\prime} \widetilde{\psi}(k) \widetilde{\psi}\left(k^{\prime}\right) \mathrm{e}^{\mathrm{i} \hbar\left(k^{2}-k^{\prime 2}\right) t / 2 m} k k^{\prime}, \quad \Omega \rightarrow \infty, \gamma \rightarrow \infty .
$$

The limit has to be applied such that $\sqrt{\gamma} / \Omega^{2} \rightarrow 0$ for $\Omega \rightarrow \infty, \gamma \rightarrow \infty$. The mean waiting time vanishes and a deconvolution is not necessary. Usual normalization leads to the same result found in Eq. (3.104), i.e. to the ideal distribution that is proportional to a local kinetic energy density. A discussion of this expression can be found in Section 3.5.

Strong decay $(\gamma \rightarrow \infty)$ : Assuming strong decay from the beginning, the driving with $\Omega$ becomes inefficient and the case is similar to the assumption of a weak laser field. For $\gamma \rightarrow \infty$, Eq. (3.125) becomes in leading order

$$
\Pi_{\delta}(t) \sim \sqrt{\frac{m}{2 \hbar \gamma}} \frac{\Omega^{2} L_{0}^{2}}{4 \pi} \int_{0}^{\infty} \mathrm{d} k \mathrm{~d} k^{\prime} \bar{\psi}(k) \widetilde{\psi}\left(k^{\prime}\right) \mathrm{e}^{\mathrm{i} \hbar\left(k^{2}-k^{\prime 2}\right) t / 2 m} \propto\left|\psi_{\mathrm{f}}(0, t)\right|^{2}, \quad \gamma \rightarrow \infty .
$$


Interestingly, the kernel is independent of $k$ and $\Pi_{\delta}(t)$ is proportional to the density of the free wave packet $\left|\psi_{\mathrm{f}}(t)\right\rangle$ of Eq. (3.105) at $x=0$. Clearly, for an antisymmetric superposition of two wave packets coming in from the left and from the right, respectively, this expression vanishes identically and it is thus not normalizable. For all other cases, a normalization of $\Pi_{\delta}(t)$ yields

$$
\Pi_{\delta, \mathrm{N}}=\frac{\left|\psi_{\mathrm{f}}(0, t)\right|^{2}}{m\left\langle k^{-1}\right\rangle / \hbar}, \quad \gamma \rightarrow \infty
$$

with $\left\langle k^{-1}\right\rangle=\int \mathrm{d} k|\widetilde{\psi}(k)|^{2} k^{-1}$, i.e. the result is the spatial probability density of the state times the inverse of the mean inverse velocity. This is closely related to the zeroth order of the expansion of Kijowski's arrival-time distribution for wave packets with well defined momentum (Eq. (3.196)). Moreover, it provides a way to measure the modulus of the wave function.

To conclude, it has been shown that the arrival-time model of this work leads to a variety of ideal expressions when considered in specific limits of the quantum optical parameters.

\subsection{Arrival-time measurement with absorbing po- tentials}

In his pioneering work, Allcock [6] concentrated on a measurement of arrival times and detector models rather than time operators or uncertainty relations. He describes a model, where the information on the arrival is transferred from the incident channel to an "apparatus channel" of the detector. The detector is heuristically modeled by a half-space absorbing potential $V(x)=-\mathrm{i} V \Theta(x)$ and the rate of the detection is assumed to be the absorbing rate, i.e. the negative change of the incident probability:

$$
\Pi_{\text {Allcock }}(t)=-\frac{\mathrm{d}}{\mathrm{d} t}\|\psi(t)\|^{2}=\frac{2 V}{\hbar} \int_{0}^{\infty} \mathrm{d} x|\psi(x, t)|^{2} .
$$

Allcock also mentioned the reflection and delay problem, and he solved it by considering the weak potential limit $V \rightarrow 0$, avoiding reflections, and rectifying the poor time resolution by a deconvolution with the apparatus resolution function.

Later, imaginary potentials have often been introduced as detector models for time measurements, apart from their importance in nuclear physics or quantumchemical simulations (see Ref. [99] and references therein). Muga et al. [7] showed that the reflection problem due to the step-potential can be partially circumvented by using appropriately constructed absorbing potentials with a real part, and that an optimization of such constructions is possible [82, 100].

Imaginary potentials have also become important for the event-enhanced quantum theory (EEQT) of Blanchard and Jadczyk [79, 80].

In the following section it is shown that the time evolution of a quantum particle, moving in one dimension under the influence of a complex-valued potential, can be considered as a limiting case of the time evolution of a moving two-level atom, 
interacting with a laser field. The advantage of this point of view is that the physical background of the laser model provides an easy understanding of the one-channel problem and in particular of the absorption mechanism. The ad hoc parameters which describe the real and the imaginary part get a physical meaning as functions of Rabi frequency, decay rate and detuning. Furthermore, it is shown that in the same limit the reset state of the atom with neglected recoil corresponds to the reset state used in the EEQT.

Besides the easy understanding in terms of a limiting case of the laser model, it has been shown very recently that the following results may be reformulated in a more general way by applying the Feshbach partitioning technique [101, 102] to the two-channel Schrödinger equation to obtain an "optical potential" [103].

\subsubsection{Absorbing potentials as a limiting case of the laser model}

The general solution of the stationary two-channel Schrödinger equation for a laser barrier $\Omega(x)=\Omega \chi_{[0, L]}(x)$ is given in Section B.2.2 of the appendix. It reads

$$
\Phi_{k}(x)=\frac{1}{\sqrt{2 \pi}}\left\{\begin{aligned}
\left(\begin{array}{c}
\mathrm{e}^{\mathrm{i} k x}+B_{0}^{+} \mathrm{e}^{-\mathrm{i} k x} \\
B_{0}^{-} \mathrm{e}^{-\mathrm{i} q x}
\end{array}\right), & x \leq 0, \\
A_{1}^{+}\left|\lambda_{+}\right\rangle \mathrm{e}^{\mathrm{i} k_{+} x}+B_{1}^{+}\left|\lambda_{+}\right\rangle \mathrm{e}^{-\mathrm{i} k_{+} x} & \\
+A_{1}^{-}\left|\lambda_{-}\right\rangle \mathrm{e}^{\mathrm{i} k_{-} x}+B_{1}^{-}\left|\lambda_{-}\right\rangle \mathrm{e}^{-\mathrm{i} k_{-} x}, & 0 \leq x \leq L, \\
\left(\begin{array}{l}
A_{2}^{+} \mathrm{e}^{\mathrm{i} k x} \\
A_{2}^{-} \mathrm{e}^{\mathrm{i} q x}
\end{array}\right), & x \geq L,
\end{aligned}\right.
$$

where $\lambda_{ \pm}$and the wave numbers $k_{ \pm}$and $q$ are given by Eqs. (3.58)-(3.60) and the amplitudes are determined by the matching conditions at $x=0$ and at $x=L$ and they are derived in Section B.2.2.

Now the limit of fast pumping $(E \ll \hbar \Omega)$ and strong decay $(E \ll \hbar|\gamma-2 \mathrm{i} \delta|)$ is considered, but with the decay even faster, such that $\Omega^{2} /|\gamma-2 \mathrm{i} \delta|=$ const. [83, 84]. 
In this limit, one has in leading order

$$
\begin{aligned}
& k_{+} \rightarrow \sqrt{k^{2}+\frac{\mathrm{i} m \Omega^{2}}{\hbar(\gamma-2 \mathrm{i} \delta)}}, \\
& k_{-} \sim q \sim \sqrt{m(\mathrm{i} \gamma+2 \delta) / \hbar} \text {, } \\
& B_{0}^{+} \rightarrow B_{0}, \quad A_{2}^{+} \rightarrow A_{2}, \quad B_{0}^{-} \rightarrow 0, \quad A_{2}^{-} \rightarrow 0, \\
& A_{1}^{+}\left|\lambda_{+}\right\rangle \rightarrow A_{1}\left(\begin{array}{c}
1 \\
-\mathrm{i} \Omega /(\gamma-2 \mathrm{i} \delta)
\end{array}\right) \\
& B_{1}^{+}\left|\lambda_{+}\right\rangle \rightarrow B_{1}\left(\begin{array}{c}
1 \\
-\mathrm{i} \Omega /(\gamma-2 \mathrm{i} \delta)
\end{array}\right) \text {, } \\
& A_{1}^{-}\left|\lambda_{-}\right\rangle \mathrm{e}^{\mathrm{i} k_{-} x} \rightarrow\left(\begin{array}{l}
0 \\
0
\end{array}\right), \quad 0 \leq x \leq L, \\
& B_{1}^{-}\left|\lambda_{-}\right\rangle \mathrm{e}^{-\mathrm{i} k_{-} x} \rightarrow\left(\begin{array}{l}
0 \\
0
\end{array}\right), \quad 0 \leq x \leq L .
\end{aligned}
$$

The limit of the wave number $k_{+}$can be written as

$$
k_{+} \rightarrow \sqrt{k^{2}-2 m V / \hbar^{2}},
$$

where the complex absorbing potential

$$
\begin{aligned}
V(x) & \equiv V \chi_{[0, L]}(x) \\
& =\frac{\hbar \Omega(x)^{2}}{2(\mathrm{i} \gamma+2 \delta)}=\frac{\hbar \Omega(x)^{2} \delta}{\gamma^{2}+4 \delta^{2}}-\frac{\mathrm{i} \hbar \gamma \Omega(x)^{2}}{2\left(\gamma^{2}+4 \delta^{2}\right)}
\end{aligned}
$$

has been defined. The coefficients $A_{i}, B_{i}$ are given by Eqs. (B.12) for the potential $V$. For large detuning, $|\delta| \gg \gamma$ the laser field acts as a real potential, whose sign can be controlled by the sign of the detuning, whereas for a resonant laser, $\delta=0$, the potential becomes purely absorbing,

$$
V(x)=-\mathrm{i} \frac{\hbar \Omega^{2}(x)}{2 \gamma} \quad \text { for } \delta=0 .
$$

It is obvious from Eqs. (3.137) that the ground state wave function equals the onechannel solution for a complex absorbing barrier potential $V$, whereas the excited state vanishes inside the laser region with $\Omega / \gamma$ and is exponentially damped outside. This can be written as

$$
\phi^{(2)}(x)=-\frac{\mathrm{i} \Omega(x)}{\gamma-2 \mathrm{i} \delta} \phi^{(1)}(x),
$$

where $\phi^{(1)}$ is the stationary solution of the one-channel Schrödinger equation

$$
\left(\frac{\widehat{p}^{2}}{2 m}+V(\widehat{x})\right) \phi^{(1)}=E_{k} \phi^{(1)}
$$

For the complex barrier potential, $V(x)=V \chi_{[0, L]}(x)$, the eigenfunctions are given in Section B.1. 
The above limit can be generalized for arbitrary laser shapes, by noting that every shape $\Omega(x)$ of the Rabi frequency is a limiting case of an infinite number of barriers with vanishing width. Each of them tends to a complex barrier potential, thus all of them lead to a continuous complex potential.

In the above limit one has for the first-photon distribution with Eq. (3.70) and for a purely absorbing potential $(\delta=0)$

$$
\begin{aligned}
\Pi(t) & =\gamma \int_{-\infty}^{\infty} \mathrm{d} x\left|\psi^{(2)}(x, t)\right|^{2} \rightarrow \frac{\Omega^{2}}{\gamma} \int_{0}^{L} \mathrm{~d} x|\psi(x, t)|^{2} \\
& \equiv \frac{2 V}{\hbar} \int_{0}^{L} \mathrm{~d} x|\psi(x, t)|^{2}, \quad \gamma \rightarrow \infty, \Omega^{2} / \gamma=\text { const. }
\end{aligned}
$$

where $\left|\psi_{t}\right\rangle=\int \mathrm{d} k \widetilde{\psi}(k) \mathrm{e}^{-\mathrm{i} \hbar k^{2} t / 2 m}\left|\phi_{k}^{(1)}\right\rangle$ is the wave packet corresponding to the onechannel evolution of Eq. (3.143). Although $\psi_{t}^{(2)} \rightarrow 0, \gamma\left|\psi_{t}^{(2)}\right|^{2}$ remains finite and the expression (3.135) of Allcock is recovered. Thus, arrival-time models which are based on absorbing potentials are a special case of the two-channel laser model. The notion of "absorption" can be understood physically as the emission of the first photon in suitable limits, thus as a "vanishing" of the undetected atom.

\subsubsection{Deconvolution of the absorption rate}

In this section it is shown that the deconvolution procedure, used in Section 3.2.3 for the laser model, also yields the quantum mechanical flux for absorbing potentials which are localized in the right half-space, $x \geq 0$. This has been first realized by Allcock [6]. To proceed, one needs the "waiting time distribution" for the absorption which can be obtained by applying the one-channel limit $\gamma \rightarrow \infty, \gamma / \Omega^{2}=$ const. to the distribution (3.77) or by noting that the time evolution of an atom at rest in an absorbing potential $H=-\mathrm{i} V$ is given by $\left|\psi_{t}\right\rangle=\mathrm{e}^{-V t / \hbar}\left|\psi_{0}\right\rangle$ and thus it follows that

$$
W_{\text {abs }}(t)=-\frac{\mathrm{d}}{\mathrm{d} t}\left\|\psi_{t}\right\|^{2}=\frac{2 V}{\hbar} \mathrm{e}^{-2 V t / \hbar} .
$$

The convolution ansatz for the absorption rate (3.144) in the limit $L \rightarrow \infty$ reads

$$
\Pi(t)=\left(\Pi_{\mathrm{id}} * W_{\mathrm{abs}}\right)(t)
$$

and it yields

$$
\widetilde{\Pi}_{\mathrm{id}}(\nu)=\frac{\widetilde{\Pi}(\nu)}{\widetilde{W}_{\mathrm{abs}}(\nu)}
$$

in the Fourier domain. In the weak potential limit, $V \rightarrow 0$, and with Eq. (3.145) one has

$$
\frac{1}{\widetilde{W}_{\text {abs }}(\nu)} \sim \frac{\mathrm{i} \nu \hbar}{2 V}
$$

The Fourier transform of $\Pi(t)$, given with Eq. (3.144), can be calculated as in Section 3.2.3. The wave packet for $x \geq 0$, i.e. in the transmitted regime, takes with 
Eqs. (B.3) and (B.17) the form

$$
\psi(x, t)=\int_{0}^{\infty} \widetilde{\psi}(k) \mathrm{e}^{-\mathrm{i} \hbar k^{2} / 2 m} \frac{2 k}{k+\kappa} \mathrm{e}^{\mathrm{i} \kappa x},
$$

and inserting this expression into Eq. (3.144) yields

$$
\widetilde{\Pi}(\nu) \sim \frac{2 V}{\hbar} \int_{0}^{\infty} \mathrm{d} k \mathrm{~d} k^{\prime} \bar{\psi}(k) \widetilde{\psi}\left(k^{\prime}\right) \delta\left(\nu-\frac{\hbar}{2 m}\left(k^{2}-k^{\prime 2}\right)\right) \frac{1}{\mathrm{i}\left(k-k^{\prime}\right)}, \quad V \rightarrow 0 .
$$

Inserting Eqs. (3.148) and (3.150) into Eq. (3.147) and going back to the time domain, one finally obtains

$$
\Pi_{\text {id }}(t)=\frac{1}{2 \pi} \int_{-\infty}^{\infty} \mathrm{d} \nu \mathrm{e}^{\mathrm{i} \nu t} \widetilde{\Pi}_{\mathrm{id}}(\nu) \rightarrow J(t, 0), \quad V \rightarrow 0,
$$

i.e. the quantum mechanical flux at $x=0$. This corresponds to the result for the laser model.

\subsubsection{The reset state for absorption}

Since the quantum jump approach provides knowledge not only about the conditional time evolution, but also about the reset state after a detection of a photon, one may ask about the form of this reset state in the one-channel limit. This question is particularly interesting, because the meaning of a reset state for an absorbing potential is unclear from a physical point of view. However, in the event-enhanced quantum theory (EEQT) $[79,80]$ one deals with such reset states and uses them to study physical events, e.g. particle tracks in a cloud chamber. In this section it is shown, that these reset states arise naturally from a limiting case of the state of a two-level atom right after the emission of a photon.

In the following, resonant driving of the laser is considered, $\delta=0$, and the recoil is neglected. This means for the atomic velocities

$$
v_{\text {atom }} \gg \frac{\hbar \omega_{21}}{m c} \text {. }
$$

For the $6^{2} P_{3 / 2}-6^{2} S_{1 / 2}$ transition of cesium $\left(m=2.2 \times 10^{-25} \mathrm{~kg}, \omega_{21}=2.2 \times 10^{15} \mathrm{~Hz}\right)$, this condition leads to velocities not smaller than $0.35 \mathrm{~cm} / \mathrm{s}$.

In this case, the density matrix right after an emission of a photon becomes with Eq. (3.41)

$$
\left\langle p\left|\mathscr{R}\left(\rho_{t}\right)\right| p^{\prime}\right\rangle=\gamma|1\rangle\langle 1|\left\langle 2\left|\rho_{t}\left(p, p^{\prime}\right)\right| 2\right\rangle,
$$

and with Eq. (3.40) one has for the unnormalized pure reset state after an emission at time $t$

$$
\left|\psi_{t}^{\mathscr{R}}\right\rangle=\sqrt{\gamma}|1\rangle\left\langle 2 \mid \boldsymbol{\Psi}_{t}\right\rangle=\sqrt{\gamma}\left|\psi_{t}^{(2)}\right\rangle|1\rangle
$$

It is given with Eqs. (3.141) and (3.142) and in position representation by

$$
\psi^{\mathscr{R}}(x, t)=-\frac{\mathrm{i} \Omega(x)}{\sqrt{\gamma}} \psi^{(1)}(x, t)=-\mathrm{i} \sqrt{\frac{2|V(x)|}{\hbar}} \psi^{(1)}(x, t) .
$$


This result turns out to be equivalent to the reset state put forward in the EEQT [80], where the function $\sqrt{2|V(x)| / \hbar}$ only has the abstract meaning of a "detector sensitivity". In contrast, the approach given here in terms of photon emissions clarifies its physical background. The reset state of Eq. (3.155) has been used in Ref. [103] for the simulation of fluorescence trajectories.

\subsubsection{Position-dependent decay rate}

In the previous sections it has been shown that the laser model simplifies to a onechannel description with a complex-valued potential in suitable limits. A different connection between imaginary potentials and quantum optical models can be given in terms of a position-dependent decay rate.

Consider a two-level atom, initially prepared in the upper level $|2\rangle$ and coming in from the far left. The free radiation field that couples to the atom is assumed to be position-dependent, such that

$$
\gamma(x)=\gamma \Theta(x)
$$

as shown schematically in Fig. 3.7. There is no laser in this model. The conditional

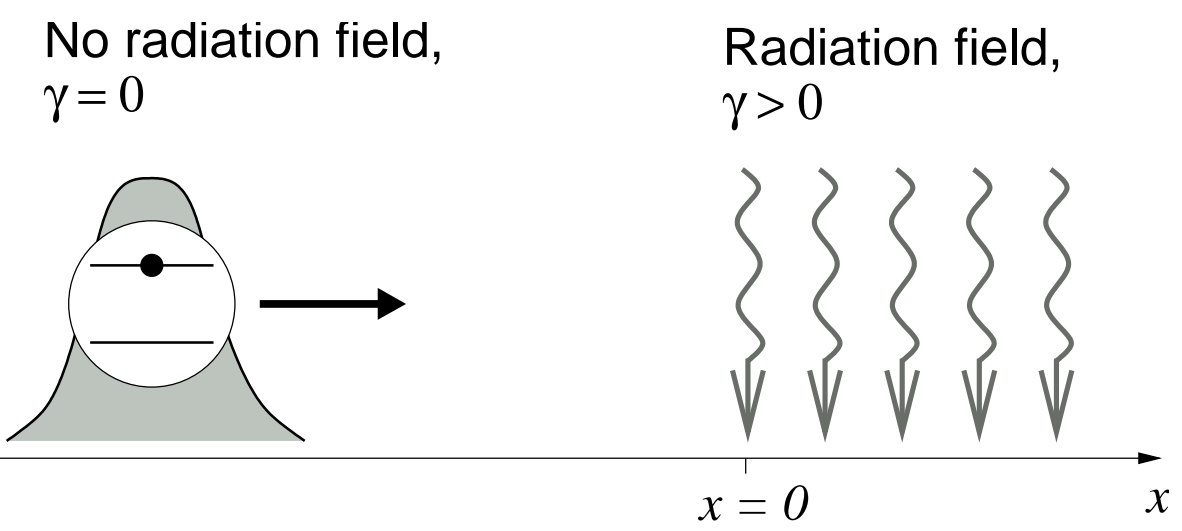

Figure 3.7: Arrival time model with position-dependent decay rate $\gamma$ in the absence of a laser field. This model is essentially equivalent to a one-channel model with imaginary potential.

time evolution is governed by the Hamiltonian

$$
H_{\mathrm{c}}=\frac{\widehat{p}^{2}}{2 m}-\frac{\mathrm{i} \hbar \gamma}{2} \Theta(\widehat{x})|2\rangle\langle 2| .
$$

The solution of the stationary Schrödinger equation $H_{\mathrm{c}} \boldsymbol{\Phi}_{k}=E_{k} \boldsymbol{\Phi}_{k}$ for the given initial condition is

$$
\boldsymbol{\Phi}_{k}(x) \equiv\left(\begin{array}{l}
\phi_{k}^{(1)}(x) \\
\phi_{k}^{(2)}(x)
\end{array}\right)=\frac{1}{\sqrt{2 \pi}} \begin{cases}\left(\begin{array}{c}
R_{1}(k) \mathrm{e}^{-\mathrm{i} k x} \\
\mathrm{e}^{\mathrm{i} k x}+R_{2}(k) \mathrm{e}^{-\mathrm{i} k x}
\end{array}\right), & x \leq 0 \\
\left(\begin{array}{c}
T_{1}(k) \mathrm{e}^{\mathrm{i} k x} \\
T_{2}(k) \mathrm{e}^{\mathrm{i} q x}
\end{array}\right), & x \geq 0,\end{cases}
$$


where

$$
q=\sqrt{k^{2}+\mathrm{i} m \gamma / \hbar}, \quad \operatorname{Im} q>0 .
$$

The reflection and transmission coefficients for this model are obtained via the matching conditions at $x=0$. For the matching of the eigenfunctions itself follows

$$
T_{1}=R_{1}, \quad T_{2}=1+R_{2}
$$

and for the matching of the first derivative one has

$$
T_{1}=-R_{1}, \quad T_{2}=\frac{k}{q}\left(1-R_{2}\right) .
$$

Solving Eqs. (3.160) and (3.161) yields

$$
\begin{aligned}
& T_{1}=R_{1}=0 \\
& R_{2}=\frac{k-q}{k+q} \\
& T_{2}=\frac{2 k}{k+q} .
\end{aligned}
$$

Thus, $\phi_{k}^{(1)}(x) \equiv 0$, and $\phi_{k}^{(2)}(x)$ is a solution of the one-channel Schrödinger equation

$$
\left(\frac{\widehat{p}^{2}}{2 m}+V(\widehat{x})\right) \phi_{k}^{(2)}=E_{k} \phi_{k}^{(2)}
$$

with the imaginary potential $V(x)=-\mathrm{i} V_{0} \Theta(x)=-\mathrm{i} \hbar \gamma \Theta(x) / 2$. A wave packet coming in from the far left with only positive momentum components is given by a superposition of scattering eigenfunctions,

$$
\psi(x, t)=\int_{0}^{\infty} \mathrm{d} k \widetilde{\psi}(k) \mathrm{e}^{-\mathrm{i} \hbar k^{2} t / 2 m} \phi_{k}^{(2)}(x)
$$

The temporal distribution of the first photon for this wave packet is given by Eq. (3.68), and with $H_{\mathrm{c}}-H_{\mathrm{c}}^{\dagger}=-\mathrm{i} \hbar \gamma \Theta(\widehat{x})$ follows

$$
\Pi(t)=\gamma \int_{0}^{\infty} \mathrm{d} x|\psi(x, t)|^{2} .
$$

This expression coincides with Eq. (3.144), since $\gamma=2 V_{0} / \hbar$. Thus it appears that models dealing with the time evolution of particles in spatially confined absorbing potentials are equivalent to two-channel models with initially excited two-level atoms which may decay solely in the corresponding spatial region.

\subsection{Arrival-time distributions and relation to ki- netic energy densities}

\subsubsection{Local kinetic energy densities}

It has been mentioned in Section 2.1.6 that an arrival time distribution can be understood as an example of a local quantum density, namely a velocity density. 
Chapter 3. Operational approaches to quantum arrival times

Local kinetic energy densities are another example which is strongly related to arrival times as it will be shown in the following.

There is no unique definition of a quantum kinetic energy density in the literature, in spite of the relevance of the concept in several fields. Kinetic energy densities are present in the Thomas-Fermi theory [104], in density functional theory to determine the electronic structure of atoms, molecules, solids or fermionic gases [105], in partitioning molecular systems into fragments with well defined energies $[106,107]$ or to define intrinsic shapes of reactants, transition states, and products along the course of a chemical reaction [108].

In analogy to Eq. (2.3), the classical expression for the phase space variable related to the local kinetic energy density at $x$ is

$$
\mathcal{K}(x)=\frac{p^{2}}{2 m} \delta(q-x) .
$$

According to Section 2.1.6, a quantization of this expression yields different possibilities for a kinetic energy density operator, for instance

$$
\begin{aligned}
\widehat{K}^{(1)}(x) & =\frac{1}{2 m} \widehat{p} \delta(\widehat{x}-x) \widehat{p} \\
\widehat{K}^{(2)}(x) & =\frac{1}{4 m}\left(\widehat{p}^{2} \delta(\widehat{x}-x)+\delta(\widehat{x}-x) \widehat{p}^{2}\right), \\
\widehat{K}^{(3)}(x) & =\frac{1}{2}\left(\widehat{K}^{(1)}(x)+\widehat{K}^{(2)}(x)\right) .
\end{aligned}
$$

The second operator follows from the quantization rule of Rivier [109]. The corresponding density $\left\langle\widehat{K}^{(2)}(x)\right\rangle_{t}$ is given by its, generally time dependent, expectation value and may in principle be obtained operationally by a weak measurement of the kinetic energy post-selected at position $x[110,111]$. The third one, which is the average of $\widehat{K}^{(1)}$ and $\widehat{K}^{(2)}$, corresponds to Weyl's quantization rule. An indirect way to measure the density $\left\langle\widehat{K}^{(3)}\right\rangle_{t}$ for free motion was described by Johansen [112], who noticed that the second time derivative of the expectation value of $|\widehat{x}-x|$ is proportional to $\left\langle\widehat{K}^{(3)}(x)\right\rangle_{t}$.

In the following it is shown that the quantum optical arrival-time model of this work provides for the first time a possibility to measure, at least in principle, the density corresponding to the first operator, $\left\langle\widehat{K}^{(1)}(x)\right\rangle_{t}$, which is the only positive one among the three examples given in Eq. (3.169)-(3.171) [113].

\subsubsection{Measurement-based approach to kinetic energy den- sities}

Laser-based model It has been shown in previous sections that the quantum optical model for arrival times provides a connection to local kinetic energy densities. Consider a laser field, either a half-space field of the form $\Omega(x)=\Omega \Theta(x)$ or a sharply localized field of the form $\Omega(x)=\Omega L_{0} \delta(x)$. For a two-level atom initially prepared in the ground state and coming in from the far left the temporal distribution for the first spontaneously emitted photon has been derived for these two cases in Section 3.2.4 
and Section 3.3.2, respectively. For a large decay rate and strong driving, $\gamma \rightarrow \infty$ and $\Omega \rightarrow \infty$, both setups yield the same result for the normalized first-photon distribution, namely

$$
\Pi_{\mathrm{N}}(t) \rightarrow \frac{\hbar}{m\langle k\rangle_{\psi}}\left\langle\psi_{\mathrm{f}}(t)|\widehat{k} \delta(\widehat{x}) \widehat{k}| \psi_{\mathrm{f}}(t)\right\rangle, \quad \gamma \rightarrow \infty, \Omega \rightarrow \infty
$$

as shown in Eqs. (3.104) and (3.132). This expression can be identified as the expectation value of $\widehat{K}^{(1)}(x=0)$ divided by the mean momentum $p_{0}=\hbar \int \mathrm{d} k k|\widetilde{\psi}(k)|^{2}$,

$$
\Pi_{\mathrm{N}}(t) \rightarrow \frac{2}{p_{0}}\left\langle\widehat{K}^{(1)}(x=0)\right\rangle_{t}, \quad \gamma \rightarrow \infty, \Omega \rightarrow \infty
$$

Note that the averages are computed with the freely moving wave function, $\left|\psi_{\mathrm{f}}(t)\right\rangle$, given by Eq. (3.105) and that the kinetic energy density at an arbitrary point $a$ is obtained by shifting the corresponding laser region.

Model with imaginary potential The previous models, a half-space laser field or a Dirac delta laser region are not as realistic as a finite rectangular laser barrier would be. Of course, the first-photon distribution for the finite laser barrier can be derived [81], but the expressions for the eigenfunctions become complicated. Therefore, the one-channel limit of Section 3.4.1 is applied in the following, where the first-photon distribution equals the absorption rate in an absorbing imaginary potential with strength $V=\hbar \Omega^{2} / 2 \gamma$ for $\gamma \rightarrow \infty$ and $\Omega^{2} / \gamma=$ const. The laser is assumed to be in resonance, $\delta=0$.

Consider the absorbing potential to be located in the interval $0 \leq x \leq L$, then the effective Hamiltonian for undetected atoms is given by

$$
H=\frac{\widehat{p}^{2}}{2 m}-\mathrm{i} V \chi_{[0, L]}(\widehat{x})
$$

and the detection rate is found with Eq. (3.144) to be

$$
\Pi(t)=\frac{2 V}{\hbar} \int_{0}^{L} \mathrm{~d} x|\psi(x, t)|^{2} .
$$

To obtain the time development of the wave packet

$$
\psi(x, t)=\int_{0}^{\infty} \mathrm{d} k \widetilde{\psi}(k) \mathrm{e}^{-\mathrm{i} \hbar k^{2} t / 2 m} \phi_{k}(x),
$$

one has to solve the stationary equation $H \phi_{k}=E_{k} \phi_{k}$. Using the transfer matrix method of Appendix B.1, the result for $\phi_{k}$ inside the absorbing potential region is

$$
\phi_{k}(x)=\frac{1}{\sqrt{2 \pi}}\left(A_{+}(k) \mathrm{e}^{\mathrm{i} q x}+A_{-}(k) \mathrm{e}^{-\mathrm{i} q x}\right),
$$

with $q^{2}=k^{2}+2 \mathrm{i} m V / \hbar$ and

$$
A_{ \pm}(k)=\frac{k(q \pm k) \mathrm{e}^{\mp \mathrm{i} q L}}{2 k q \cos (q L)-\mathrm{i}\left(k^{2}+q^{2}\right) \sin (q L)} .
$$


To relate the absorption rate $\Pi(t)$ to an ideal distribution, one can apply the strong detection limit $V \rightarrow \infty$, since this eliminates an absorption delay. When $V$ is increased, more and more atoms are reflected without being detected, but by normalizing the result a finite distribution is obtained in the limit, even though the absorption probability eventually vanishes due to total reflection. For large $V, V \gg \hbar^{2} k^{2} / 2 m$, one has in leading order

$$
\begin{aligned}
q & \sim \sqrt{2 \mathrm{i} m V / \hbar^{2}}, \\
A_{+} & \sim \frac{2 k}{q}, \\
A_{-} & \sim \frac{2 k}{q} \mathrm{e}^{2 \mathrm{i} q L}, \\
\phi_{k}(x) & \sim \frac{1}{\sqrt{2 \pi}} \frac{2 k}{q}\left(\mathrm{e}^{\mathrm{i} q x}+\mathrm{e}^{\mathrm{i} q(2 L-x)}\right), \quad 0 \leq x \leq L .
\end{aligned}
$$

Integrating over $x$ and neglecting the terms which vanish exponentially, the absorption rate becomes in leading order

$$
\Pi(t) \sim \frac{\hbar^{2}}{\pi m \sqrt{m V}} \int_{0}^{\infty} \mathrm{d} k \mathrm{~d} k^{\prime} \bar{\psi}(k) \widetilde{\psi}\left(k^{\prime}\right) \mathrm{e}^{\mathrm{i} \hbar\left(k^{2}-k^{\prime 2}\right) t / 2 m} k k^{\prime}
$$

This expression is independent of the barrier length $L$ as a result of the large $V$ limit, so the same result is obtained with an imaginary step potential $-\mathrm{i} V \Theta(\widehat{x})$ or with a very narrow barrier - as it was the case for the laser model. Finally, a normalization of Eq. (3.180) leads to the same result as in Eq. (3.172), i.e. one has

$$
\lim _{V \rightarrow \infty} \Pi_{\mathrm{N}}(t)=\frac{2}{p_{0}}\left\langle\widehat{K}^{(1)}(x=0)\right\rangle_{t} .
$$

As before, the local kinetic energy density at an arbitrary point $a$ is obtained by shifting the absorbing potential in the Hamiltonian (3.174) to $[a, a+L]$.

In Fig. 3.8, operational and ideal kinetic energy densities are compared for the paradigmatic example of a coherent superposition of two Gaussian wave packets with different momenta which are prepared in such a way that their centers of mass arrive simultaneously at the origin. This enhances the interference among different momentum components and the differences between the distributions. As seen in the figure, the differences between various versions of the quantum kinetic energy density may be quite significant. While $\left\langle\widehat{K}^{(1)}(x)\right\rangle_{t}$ is always positive, $\left\langle\widehat{K}^{(2)}(x)\right\rangle_{t}$ can become negative in classically forbidden regions for stationary eigenstates of the Hamiltonian, a fact that has been used by Tachibana [108] to define molecular and reaction shapes. It is perhaps less obvious that this quantity can also be negative as a result of free motion dynamics, as seen in the figure.

\subsubsection{The case of fixed decay rate}

In the last preceding section the ideal limits to obtain local kinetic energy densities were always connected with a change of the decay rate $\gamma$, for the laser model as 


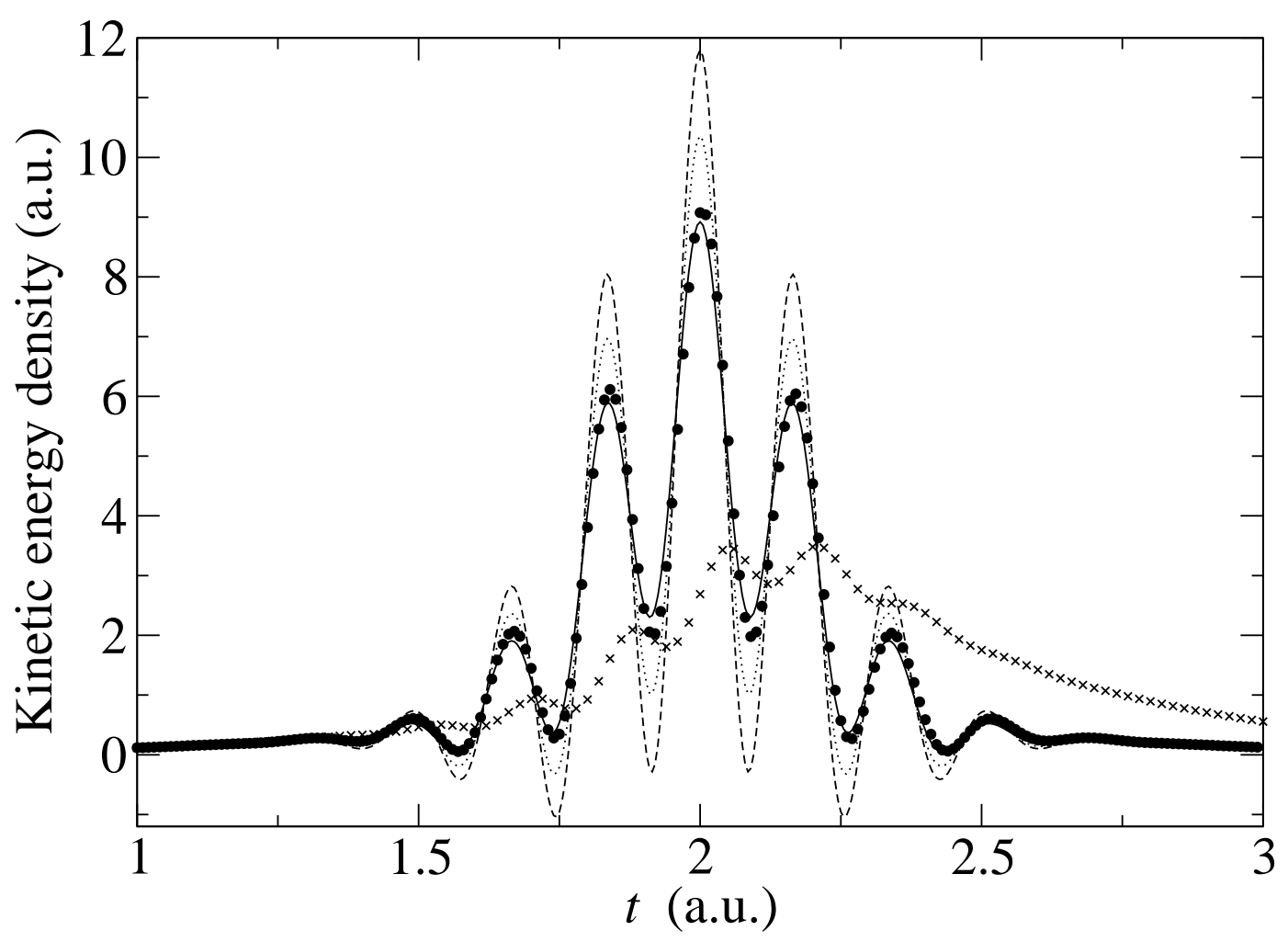

Figure 3.8: Comparison of kinetic energy densities at $x=0:\left\langle\widehat{K}^{(1)}\right\rangle_{t}$ (solid), $\left\langle\widehat{K}^{(2)}\right\rangle_{t}$ (dashed), $\left\langle\widehat{K}^{(3)}\right\rangle_{t}$ (dotted) and the operational quantity $p_{0} \Pi_{\mathrm{N}}(t) / 2$ of Eq. (3.181), for $V=2, L=10$ (crosses) and $V=500, L=20$ (circles). The initial wave packet is a coherent combination $\psi=2^{-1 / 2}\left(\psi_{1}+\psi_{2}\right)$ of two Gaussian states for the center-ofmass motion of a single cesium atom that become separately minimum-uncertainty packets (with $\Delta x_{1}=\Delta x_{2}=1.45$, and average velocities $\langle v\rangle_{1}=8.9,\langle v\rangle_{2}=2.5$ at $x=0$ and $t=2)$. One has $p_{0}=5.7$. All numbers are in atomic units.

well as for the absorbing potential, since in the latter case the limit $V \rightarrow \infty$ in connection with $\Omega^{2} / \gamma=$ const. has to be performed. Experimentally, the Rabi frequency $\Omega$ is easy to adjust, in contrast to the decay rate $\gamma$. To overcome this problem, a procedure is described in this section that allows to keep the value of $\gamma$ fixed.

For that purpose, one considers again the half-space laser model but now for the limit $\Omega \rightarrow \infty$ and $\gamma=$ const. For the derivation of the normalized first-photon distribution $\Pi_{\mathrm{N}}(t)$ which is given by Eqs. (3.70) and (3.93), one has for the leading order of the required parameters

$$
\begin{aligned}
\lambda_{ \pm} & \sim \mp \frac{\Omega}{2}-\frac{\mathrm{i} \gamma}{4}, \\
q & =\sqrt{k^{2}+\mathrm{i} m \gamma / \hbar}, \quad \operatorname{Im} q>0,
\end{aligned}
$$




$$
\begin{aligned}
& k_{ \pm} \sim \sqrt{ \pm \frac{m \Omega}{\hbar}} \pm \frac{1}{2}\left(k^{2}+\frac{\mathrm{i} m \gamma}{2 \hbar}\right) \sqrt{\frac{ \pm \hbar}{m \Omega}} \\
& R_{1} \sim-1+(1-\mathrm{i}) k \sqrt{\frac{\hbar}{m \Omega}} \\
& R_{2} \sim-(1+\mathrm{i}) k \sqrt{\frac{\hbar}{m \Omega}}
\end{aligned}
$$

This yields with Eq. (3.57)

$$
\begin{aligned}
\gamma \overline{\phi_{k}^{(2)}(x)} \phi_{k^{\prime}}^{(2)}(x) \sim \frac{\hbar \gamma}{2 \pi m} \frac{k k^{\prime}}{\Omega}\{\Theta & (-x) 2 \mathrm{e}^{\mathrm{i}\left(\bar{q}-q^{\prime}\right) x} \\
& \left.+\Theta(x)\left(-\mathrm{ie}^{-\mathrm{i} \bar{k}_{+} x}-\mathrm{e}^{-\mathrm{i} \bar{k}_{-} x}\right)\left(\mathrm{ie}^{\mathrm{i} k_{+}^{\prime} x}-\mathrm{e}^{\mathrm{i} k_{-}^{\prime} x}\right)\right\} .
\end{aligned}
$$

When integrating this expression over $x$, only the term $\mathrm{e}^{-\mathrm{i}\left(\bar{k}_{+}-k_{+}^{\prime}\right) x}$ contributes in leading order of $\Omega$. Inserting Eq. (3.187) into Eq. (3.70) and normalizing the result regarding to Eq. (3.93) leads to

$$
\Pi_{\mathrm{N}}(t) \rightarrow \frac{\hbar}{2 \pi m k_{0}} \int_{0}^{\infty} \mathrm{d} k \mathrm{~d} k^{\prime} \overline{\widetilde{\psi}(k)} \widetilde{\psi}\left(k^{\prime}\right) \mathrm{e}^{\mathrm{i} \frac{\hbar}{2 m}\left(k^{2}-k^{\prime 2}\right) t} \frac{\gamma k k^{\prime}}{\gamma+\mathrm{i} \hbar\left(k^{2}-k^{\prime 2}\right) / m} .
$$

For $\hbar \gamma$ being large compared to the kinetic energy of the incident atom, Eq. (3.104) is recovered, but for finite $\gamma$ there is a delay in the detection rate. This can be eliminated by means of a deconvolution with the first-photon distribution $W(t)$ for an atom at rest as in Section 3.2.3. The convolution ansatz

$$
\Pi_{\mathrm{N}}(t)=\Pi_{\mathrm{id}}(t) * W(t)
$$

yields in terms of Fourier transforms

$$
\widetilde{\Pi}_{\mathrm{id}}(\nu)=\frac{\widetilde{\Pi}_{\mathrm{N}}(\nu)}{\widetilde{W}(\nu)}
$$

with [96]

$$
\begin{aligned}
\frac{1}{\widetilde{W}(\nu)} & =1+\left(\frac{\gamma}{\Omega^{2}}+\frac{2}{\gamma}\right) \mathrm{i} \nu+\frac{3}{\Omega^{2}}(\mathrm{i} \nu)^{2}+\frac{2}{\gamma \Omega^{2}}(\mathrm{i} \nu)^{3} \\
& \rightarrow 1+\frac{2 \mathrm{i} \nu}{\gamma}, \quad \Omega \rightarrow \infty .
\end{aligned}
$$

Inserting this and the Fourier transform of Eq. (3.188) into Eq. (3.190), the resulting ideal distribution reads after performing the inverse Fourier transformation

$$
\Pi_{\text {id }}(t) \rightarrow \frac{\hbar}{2 \pi m k_{0}} \int_{0}^{\infty} \mathrm{d} k \int_{0}^{\infty} \mathrm{d} k^{\prime} \overline{\widetilde{\psi}(k)} \widetilde{\psi}\left(k^{\prime}\right) \mathrm{e}^{\mathrm{i} \hbar\left(k^{2}-k^{\prime 2}\right) t / 2 m} k k^{\prime}, \quad V \rightarrow \infty,
$$

which is the same expression as the normalized first-photon distribution of Eq. (3.172), obtained here operationally for fixed $\gamma$. Naturally,

$$
\lim _{V \rightarrow \infty} \Pi_{\mathrm{id}}(t)=\frac{2}{p_{0}}\left\langle\widehat{K}^{(1)}(x=0)\right\rangle_{t}
$$

holds as before. 


\subsubsection{Expansion of Kijowski's distribution}

For the operators of quantum local densities an intrinsic non-uniqueness arises due to different symmetrization possibilities, as it has been shown in Sections 2.1.6 and 3.5.1 for the cases of arrival times and kinetic energy densities. This leads to different proposals for the corresponding densities, as for instance $J(t, x)$ and $\Pi_{\mathrm{K}}(t, x)$ in the case of local velocity densities or $\left\langle\widehat{K}^{(1)}(x)\right\rangle_{t}$ and $\left\langle\widehat{K}^{(2)}(x)\right\rangle_{t}$ in the case of kinetic energy densities.

A remarkable relation between all of these quantities can be given by means of an expansion of Kijowski's distribution $\Pi_{K}(t, x)$. This also clarifies the extent of agreement between different symmetrizations for particular quantum states [113]. A related derivation, but only for arrival times, has been presented in Ref. [46].

Kijowski's distribution for arrivals at $x$ and for wave packets with only positive momentum components is given by Eq. (2.37) and it reads

$$
\Pi_{\mathrm{K}}(t)=\frac{1}{m}\left\langle\psi_{t}\left|\widehat{p}^{1 / 2}\right| x\right\rangle\left\langle x\left|\widehat{p}^{1 / 2}\right| \psi_{t}\right\rangle .
$$

For wave packets peaked around some $p_{0}=m v_{0}$ in momentum space, the operator $\widehat{p}^{1 / 2}$ acting on $\left|\psi_{t}\right\rangle$ in Eq. (3.194) can be expanded in terms of $\left(\widehat{p}-p_{0}\right)$,

$$
\widehat{p}^{1 / 2}=p_{0}^{1 / 2}+\frac{1}{2} p_{0}^{-1 / 2}\left(\widehat{p}-p_{0}\right)-\frac{1}{8} p_{0}^{-3 / 2}\left(\widehat{p}-p_{0}\right)^{2}+\mathcal{O}\left(\left(\widehat{p}-p_{0}\right)^{3}\right) .
$$

In the following one takes $p_{0}$ to be the first moment of the momentum distribution, $p_{0} / \hbar=\int \mathrm{d} k k|\widetilde{\psi}(k)|^{2}$. Inserting the expansion into Eq. (3.194) yields in zeroth order the particle density times the average velocity,

$$
\Pi_{\mathrm{K}}(t, x)=v_{0}|\psi(x, t)|^{2}+\mathcal{O}\left(\widehat{p}-p_{0}\right),
$$

which is a very intuitive result for an arrival-time distribution, though it is shown here to agree with Kijowski's distribution only for quasi-monochromatic wave packets. To first order in $\left(\widehat{p}-p_{0}\right)$ one obtains the flux at $x$,

$$
\Pi_{\mathrm{K}}(t, x)=J(t, x)+\mathcal{O}\left(\left(\widehat{p}-p_{0}\right)^{2}\right),
$$

and to second order

$$
\Pi_{\mathrm{K}}(t, x)=J(t, x)+\frac{1}{2 p_{0}}\left\langle\widehat{K}^{(1)}(x)-\widehat{K}^{(2)}(x)\right\rangle_{t}+\mathcal{O}\left(\left(\widehat{p}-p_{0}\right)^{3}\right),
$$

where $\widehat{K}^{(1)}(x)$ and $\widehat{K}^{(2)}(x)$ are the two different versions of local kinetic energy density operators given in Eqs. (3.169) and (3.170). This result means that the usual quantum mechanical flux and Kijowski's distribution coincide for sharply peaked momentum distributions. For states with only positive momenta, the time integral of the flux is correctly normalized to 1 , and so is the second order since the time integral over $\Delta(x, t)=\left\langle\widehat{K}^{(1)}(x)-\widehat{K}^{(2)}(x)\right\rangle_{t}$ is easily shown to vanish, so that this difference only provides a local-in-time correction to $J$ that averages out globally. 
Note that the difference of $\left\langle\widehat{K}^{(1)}(x)\right\rangle_{t}$ and $\left\langle\widehat{K}^{(2)}(x)\right\rangle_{t}$ has a similar meaning as the difference between Kijowski's arrival-time distribution and the quantum mechanical flux as shown in Eq. (3.198). Therefore the condition $\Delta(x, t)=\left\langle\widehat{K}^{(1)}(x)-\right.$ $\left.\widehat{K}^{(2)}(x)\right\rangle_{t}=0$ implies a certain "classicality" or coalescence of the multiple quantum possibilities, for kinetic energy densities as well as for arrival-time distributions. The quantity $\Delta(x, t)$ plays a major role in Bader's theory to separate molecules into meaningful fragments [106] and its investigation has been suggested for future research in a recent review [105]. With the operational approach to $\left\langle\widehat{K}^{(1)}(x)\right\rangle_{t}$ given in this section a first measurement-based understanding of this important quantity has been obtained. 


\section{Chapter 4}

\section{Operational approach to quantum dwell times}

In this chapter, some preliminary ideas concerning an operational understanding of dwell-time distributions are collected. It has been shown in Section 2.2 that the difficulties of defining an ideal distribution are present for dwell times as well as for arrival times. This reveals the need for measurement-based approaches, especially since no operational approach to dwell-time distributions has been found so far, cf. Ref. [76] for a recent discussion.

First, in Section 4.1 the operational method to mean dwell times of Golub et $a l$. is briefly reviewed. Next, a new relation between the ideal dwell-time distribution $\Pi_{\mathrm{D}}(t)$ of Eq. (2.67) and quantum flux-flux correlation functions is derived in Section 4.2 for the free-motion case. This important result suggests the use of the flux-flux correlation as an approximation of $\Pi_{\mathrm{D}}(t)$. As an outlook, Section 4.3 presents a first idea concerning an understanding of the flux-flux correlation function in terms of photon-photon correlation functions which can be operationally obtained by means of first-photon measurements.

\subsection{Operational approach to mean dwell times}

An operational understanding of the mean dwell time for wave packets with positive momentum support has been given by Golub et al. [78]. An extended version of the model has been put forward in Ref. [114]. The mean dwell time for $\widetilde{\psi}(-k)=0, k>0$ is given with Eq. (2.73) by

$$
\left\langle\widehat{T}_{\mathrm{D}}\right\rangle=\int_{0}^{\infty} \mathrm{d} k \frac{m L}{\hbar k}|\widetilde{\psi}(k)|^{2}
$$

The authors of Ref. [78] used the absorption probability $A(k)=1-|R(k)|^{2}-|T(k)|^{2}$ in a rectangular imaginary potential $V(x)=-\mathrm{i} V_{0} \chi_{\left[x_{1}, x_{2}\right]}(x)$ that covers the dwell interval $x_{1} \leq x \leq x_{2}$ as an operational quantity. They show that the following result holds in the weak-potential limit:

$$
\lim _{V_{0} \rightarrow 0} \frac{\hbar}{2} \frac{\partial}{\partial V_{0}} \int_{0}^{\infty} \mathrm{d} k|\widetilde{\psi}(k)|^{2} A(k)=\left\langle\widehat{T}_{\mathrm{D}}\right\rangle
$$


Thus, the change of the total absorption probability with the potential strength $V_{0}$ in the weak-potential limit equals the mean dwell time for particles coming in from the left. Note that this limit is the same as the one that has been used to obtain the flux from the absorption rate in Section 3.4.2.

\subsection{Quantum dwell-time distributions and flux- flux correlation functions}

\subsubsection{Quantum flux-flux correlations}

An alternative understanding of the dwell-time distribution (2.67) or at least of its first and second moment can be given by a quantization of the classical flux-flux correlation function of Section 2.2.1. A similar procedure has been described in Ref. [65], but within a micro-canonical picture and without considering the second moment which is the important quantity since $\left\langle\widehat{T}_{\mathrm{D}}^{2}\right\rangle$ reflects the quantum features of the distribution.

One starts with a quantization of the classical flux-flux correlation function in Eq. (2.54) by defining the operator

$$
\begin{aligned}
\widehat{C}\left(x_{1}, x_{2} ; t, \tau\right)=\widehat{J}\left(x_{2}, t+\tau\right) \widehat{J}( & \left.x_{1}, t\right)+\widehat{J}\left(x_{1}, t+\tau\right) \widehat{J}\left(x_{2}, t\right) \\
& -\widehat{J}\left(x_{1}, t+\tau\right) \widehat{J}\left(x_{1}, t\right)-\widehat{J}\left(x_{2}, t+\tau\right) \widehat{J}\left(x_{2}, t\right),
\end{aligned}
$$

where $\widehat{J}(x, t)$ is the usual quantum mechanical flux operator in the Heisenberg picture with respect to the free Hamiltonian $H=\widehat{p}^{2} / 2 m$,

$$
\widehat{J}(x, t)=\mathrm{e}^{\mathrm{i} H t / \hbar} \frac{\hbar}{2 m}(\widehat{k}|x\rangle\langle x|+| x\rangle\langle x| \widehat{k}) \mathrm{e}^{-\mathrm{i} H t / \hbar} .
$$

Furthermore, symmetrization of this operator is required which leads to

$$
\frac{1}{2}\left(\widehat{C}\left(x_{1}, x_{2} ; t, \tau\right)+\widehat{C}^{\dagger}\left(x_{1}, x_{2} ; t, \tau\right)\right)=\operatorname{Re} \widehat{C}\left(x_{1}, x_{2} ; t, \tau\right) .
$$

Following Section 2.2.1, one has to integrate the expectation value of this operator over $t$ and may then calculate the first (or second) moment with respect to $\tau$. Firstly, the following correlation function shall be derived:

$$
C(\tau)=\left\langle\psi\left|\int_{-\infty}^{\infty} \mathrm{d} t \operatorname{Re} \widehat{C}\left(x_{1}, x_{2} ; t, \tau\right)\right| \psi\right\rangle
$$

Inserting $\widehat{C}$ from Eq. (4.3) and using $J_{k k^{\prime}}(x)=\left\langle k|\widehat{J}(x, 0)| k^{\prime}\right\rangle$ one has in the $k$-space

$$
\begin{aligned}
& C(\tau)=\frac{\hbar^{2}}{4 m^{2}} \operatorname{Re} \int_{-\infty}^{\infty} \mathrm{d} k \mathrm{~d} k^{\prime} \mathrm{d} k^{\prime \prime} \int_{-\infty}^{\infty} \mathrm{d} t \overline{\widetilde{\psi}(k)} \widetilde{\psi}\left(k^{\prime}\right) \mathrm{e}^{\mathrm{i} \hbar\left(k^{2}-k^{\prime 2}\right) t / 2 m} \mathrm{e}^{\mathrm{i} \hbar\left(k^{2}-k^{\prime \prime 2}\right) \tau / 2 m} \\
& \times\left(J_{k k^{\prime \prime}}\left(x_{2}\right) J_{k^{\prime \prime} k^{\prime}}\left(x_{1}\right)+J_{k k^{\prime \prime}}\left(x_{1}\right) J_{k^{\prime \prime} k^{\prime}}\left(x_{2}\right)-J_{k k^{\prime \prime}}\left(x_{1}\right) J_{k^{\prime \prime} k^{\prime}}\left(x_{1}\right)-J_{k k^{\prime \prime}}\left(x_{2}\right) J_{k^{\prime \prime} k^{\prime}}\left(x_{2}\right)\right),
\end{aligned}
$$


where the term in brackets can be written as

$$
-\left(J_{k k^{\prime \prime}}\left(x_{2}\right)-J_{k k^{\prime \prime}}\left(x_{1}\right)\right)\left(J_{k^{\prime \prime} k^{\prime}}\left(x_{2}\right)-J_{k^{\prime \prime} k^{\prime}}\left(x_{1}\right)\right)
$$

and the flux differences are given by

$$
\begin{aligned}
J_{k k^{\prime \prime}}\left(x_{2}\right)-J_{k k^{\prime \prime}}\left(x_{1}\right) & =\int_{x_{1}}^{x_{2}} \mathrm{~d} x \frac{\mathrm{d}}{\mathrm{d} x} J_{k k^{\prime \prime}}(x) \\
& =\frac{\mathrm{i}}{2 \pi}\left(k^{\prime \prime 2}-k^{2}\right) \int_{x_{1}}^{x_{2}} \mathrm{~d} x \mathrm{e}^{\mathrm{i}\left(k^{\prime \prime}-k\right) x} .
\end{aligned}
$$

Inserting Eqs. (4.8) and (4.9) into Eq. (4.7) and performing the $t$-integration leads to a sum of two delta distributions and finally to

$$
\begin{aligned}
& C(\tau)=-\frac{\hbar}{8 \pi m} \operatorname{Re} \int_{-\infty}^{\infty} \mathrm{d} k \mathrm{~d} k^{\prime \prime} \frac{\left(k^{\prime \prime 2}-k^{2}\right)^{2}}{|k|} \mathrm{e}^{\mathrm{i} \hbar\left(k^{2}-k^{\prime \prime 2}\right) \tau / 2 m} \\
& \times\left[|\widetilde{\psi}(k)|^{2} \int_{x_{1}}^{x_{2}} \mathrm{~d} x \mathrm{~d} x^{\prime} \mathrm{e}^{\mathrm{i}\left(k^{\prime \prime}-k\right)\left(x-x^{\prime}\right)}+\overline{\widetilde{\psi}(k)} \widetilde{\psi}(-k) \int_{x_{1}}^{x_{2}} \mathrm{~d} x \mathrm{~d} x^{\prime} \mathrm{e}^{\mathrm{i}\left(k^{\prime \prime}-k\right) x} \mathrm{e}^{-\mathrm{i}\left(k^{\prime \prime}+k\right) x^{\prime}}\right] .
\end{aligned}
$$

Note that $C(\tau)$ is not necessarily positive, in fact its negative contributions cancel its positive contributions, since $\int_{0}^{\infty} \mathrm{d} \tau C(\tau)=0$ :

$$
\begin{aligned}
& \int_{0}^{\infty} \mathrm{d} \tau C(\tau)=-\frac{1}{4 \pi} \operatorname{Re} \int_{-\infty}^{\infty} \mathrm{d} k \mathrm{~d} k^{\prime \prime} \frac{\left(k^{\prime \prime 2}-k^{2}\right)^{2}}{|k|}\left(\pi \delta\left(k^{2}-k^{\prime \prime 2}\right)+\mathrm{i} \mathcal{P} \frac{1}{k^{2}-k^{\prime \prime 2}}\right) \\
& \times\left[|\widetilde{\psi}(k)|^{2} \int_{x_{1}}^{x_{2}} \mathrm{~d} x \mathrm{~d} x^{\prime} \mathrm{e}^{\mathrm{i}\left(k^{\prime \prime}-k\right)\left(x-x^{\prime}\right)}\right. \\
& \left.+\overline{\widetilde{\psi}(k)} \widetilde{\psi}(-k) \int_{x_{1}}^{x_{2}} \mathrm{~d} x \mathrm{~d} x^{\prime} \mathrm{e}^{\mathrm{i}\left(k^{\prime \prime}-k\right) x} \mathrm{e}^{-\mathrm{i}\left(k^{\prime \prime}+k\right) x^{\prime}}\right] \\
& =-\frac{1}{4 \pi} \operatorname{Im} \int_{-\infty}^{\infty} \mathrm{d} k \mathrm{~d} k^{\prime \prime} \frac{\left(k^{\prime \prime 2}-k^{2}\right)}{|k|}\left[|\widetilde{\psi}(k)|^{2}\left|\int_{x_{1}}^{x_{2}} \mathrm{~d} x \mathrm{e}^{\mathrm{i}\left(k^{\prime \prime}-k\right) x}\right|^{2}\right. \\
& \left.+\overline{\widetilde{\psi}(k)} \widetilde{\psi}(-k) \int_{x_{1}}^{x_{2}} \mathrm{~d} x \mathrm{~d} x^{\prime} \mathrm{e}^{\mathrm{i}\left(k^{\prime \prime}-k\right) x} \mathrm{e}^{-\mathrm{i}\left(k^{\prime \prime}+k\right) x^{\prime}}\right] .
\end{aligned}
$$

The first part of the integrand is obviously real and thus vanishes. For the second 
part follows:

$$
\begin{aligned}
& \operatorname{Im} \int_{-\infty}^{\infty} \mathrm{d} k \mathrm{~d} k^{\prime \prime} \bar{\psi}(k) \widetilde{\psi}(-k) \int_{x_{1}}^{x_{2}} \mathrm{~d} x \mathrm{~d} x^{\prime} \mathrm{e}^{\mathrm{i}\left(k^{\prime \prime}-k\right) x} \mathrm{e}^{-\mathrm{i}\left(k^{\prime \prime}+k\right) x^{\prime}} \\
& =\frac{1}{2 \mathrm{i}}\left(\int_{-\infty}^{\infty} \mathrm{d} k \mathrm{~d} k^{\prime \prime} \bar{\psi}(k) \widetilde{\psi}(-k) \int_{x_{1}}^{x_{2}} \mathrm{~d} x \mathrm{~d} x^{\prime} \mathrm{e}^{\mathrm{i}\left(k^{\prime \prime}-k\right) x} \mathrm{e}^{-\mathrm{i}\left(k^{\prime \prime}+k\right) x^{\prime}}\right. \\
& \left.-\int_{-\infty}^{\infty} \mathrm{d} k \mathrm{~d} k^{\prime \prime} \widetilde{\psi}(k) \overline{\widetilde{\psi}(-k)} \int_{x_{1}}^{x_{2}} \mathrm{~d} x \mathrm{~d} x^{\prime} \mathrm{e}^{-\mathrm{i}\left(k^{\prime \prime}-k\right) x} \mathrm{e}^{\mathrm{i}\left(k^{\prime \prime}+k\right) x^{\prime}}\right) \\
& =\frac{1}{2 \mathrm{i}}\left(\int_{-\infty}^{\infty} \mathrm{d} k \mathrm{~d} k^{\prime \prime} \overline{\widetilde{\psi}(k)} \widetilde{\psi}(-k) \int_{x_{1}}^{x_{2}} \mathrm{~d} x \mathrm{~d} x^{\prime} \mathrm{e}^{\mathrm{i}\left(k^{\prime \prime}-k\right) x} \mathrm{e}^{-\mathrm{i}\left(k^{\prime \prime}+k\right) x^{\prime}}\right. \\
& \left.-\int_{-\infty}^{\infty} \mathrm{d} k \mathrm{~d} k^{\prime \prime} \widetilde{\psi}(-k) \overline{\widetilde{\psi}(k)} \int_{x_{1}}^{x_{2}} \mathrm{~d} x \mathrm{~d} x^{\prime} \mathrm{e}^{-\mathrm{i}\left(k^{\prime \prime}+k\right) x} \mathrm{e}^{\mathrm{i}\left(k^{\prime \prime}-k\right) x^{\prime}}\right) \\
& =0 \text {. }
\end{aligned}
$$

In the second term of Eq. (4.12), $x$ and $x^{\prime}$ may be interchanged and $k$ has been substituted by $-k$. Thus, the statement follows.

\subsubsection{First moment}

The first moment of $C(\tau)$ is given by $\int_{0}^{\infty} \mathrm{d} \tau \tau C(\tau)$. The $\tau$-integration results in

$$
\begin{aligned}
\int_{0}^{\infty} \mathrm{d} \tau \tau \mathrm{e}^{\mathrm{i} \hbar\left(k^{2}-k^{\prime \prime 2}\right) \tau / 2 m} & =\frac{\mathrm{i} m}{\hbar k^{\prime \prime}} \frac{\partial}{\partial k^{\prime \prime}} \int_{0}^{\infty} \mathrm{d} \tau \mathrm{e}^{\mathrm{i} \hbar\left(k^{2}-k^{\prime \prime 2}\right) \tau / 2 m} \\
& =-\frac{2 m^{2}}{\hbar^{2} k^{\prime \prime}} \frac{\partial}{\partial k^{\prime \prime}}\left(\mathcal{P} \frac{1}{k^{2}-k^{\prime \prime 2}}-\mathrm{i} \pi \delta\left(k^{2}-k^{\prime \prime 2}\right)\right) .
\end{aligned}
$$

By inserting this into Eq. (4.10) and by evaluating the real part a sum of three terms is obtained,

$$
\begin{aligned}
& \int_{0}^{\infty} \mathrm{d} \tau \tau C(\tau) \\
& =\frac{m}{4 \pi \hbar} \int_{-\infty}^{\infty} \mathrm{d} k \mathrm{~d} k^{\prime \prime}\left(\frac{\partial}{\partial k^{\prime \prime}} \frac{1}{k^{2}-k^{\prime \prime 2}}\right) \frac{\left(k^{\prime \prime 2}-k^{2}\right)^{2}}{k^{\prime \prime}|k|}|\widetilde{\psi}(k)|^{2}\left|\int_{x_{1}}^{x_{2}} \mathrm{~d} x \mathrm{e}^{\mathrm{i}\left(k^{\prime \prime}-k\right) x}\right|^{2} \\
& +\frac{m}{4 \pi \hbar} \int_{-\infty}^{\infty} \mathrm{d} k \mathrm{~d} k^{\prime \prime}\left(\frac{\partial}{\partial k^{\prime \prime}} \frac{1}{k^{2}-k^{\prime \prime 2}}\right) \frac{\left(k^{\prime \prime 2}-k^{2}\right)^{2}}{k^{\prime \prime}|k|} \\
& \quad \times \operatorname{Re}\left\{\widetilde{\psi}(k) \widetilde{\psi}(-k) \int_{x_{1}}^{x_{2}} \mathrm{~d} x \mathrm{~d} x^{\prime} \mathrm{e}^{\mathrm{i}\left(k^{\prime \prime}-k\right) x} \mathrm{e}^{-\mathrm{i}\left(k^{\prime \prime}+k\right) x^{\prime}}\right\} \\
& -\frac{m}{4 \pi \hbar} \int_{-\infty}^{\infty} \mathrm{d} k \mathrm{~d} k^{\prime \prime}\left(\frac{\partial}{\partial k^{\prime \prime}} \pi \delta\left(k^{\prime \prime 2}-k^{2}\right)\right) \frac{\left(k^{\prime \prime 2}-k^{2}\right)^{2}}{k^{\prime \prime}|k|} \\
& \quad \times \operatorname{Im}\left\{\widetilde{\widetilde{\psi}(k)} \widetilde{\psi}(-k) \int_{x_{1}}^{x_{2}} \mathrm{~d} x \mathrm{~d} x^{\prime} \mathrm{e}^{\mathrm{i}\left(k^{\prime \prime}-k\right) x} \mathrm{e}^{-\mathrm{i}\left(k^{\prime \prime}+k\right) x^{\prime}}\right\} .
\end{aligned}
$$

The third term including the delta distribution vanishes according to Eq. (4.12). The first and the second terms of Eq. (4.15) are easily derived, since the $k^{\prime \prime}$-integration 
can be directly performed, as well as the integrals over the dwell region. Using Eq. (4.12), the resulting expression is

$$
\int_{0}^{\infty} \mathrm{d} \tau \tau C(\tau)=\int_{-\infty}^{\infty} d k \frac{m L}{\hbar|k|}\left(|\widetilde{\psi}(k)|^{2}+\overline{\widetilde{\psi}(k)} \widetilde{\psi}(-k) \frac{\sin (k L) \mathrm{e}^{-\mathrm{i} k\left(x_{1}+x_{2}\right)}}{k L}\right)
$$

This equals the first moment of the ideal dwell-time distribution derived in Eq. (2.73).

\subsubsection{Second moment}

A similar derivation is now performed for the second moment of the flux-flux correlation function which is given by $\int_{0}^{\infty} \mathrm{d} \tau \tau^{2} C(\tau)$. The corresponding calculation according to Eq. (4.14) is given by

$$
\begin{aligned}
& \int_{0}^{\infty} \mathrm{d} \tau \tau^{2} \mathrm{e}^{\mathrm{i} \hbar\left(k^{2}-k^{\prime \prime 2}\right) \tau / 2 m}=-\frac{8 m^{3}}{\hbar^{3}} \frac{\partial^{2}}{\left(\partial k^{\prime \prime 2}\right)^{2}}\left(\mathrm{i} \mathcal{P} \frac{1}{k^{2}-k^{\prime \prime 2}}+\pi \delta\left(k^{2}-k^{\prime \prime 2}\right)\right) \\
&=-\frac{2 m^{3}}{\hbar^{3}} \frac{1}{k^{\prime \prime}}\left(-\frac{1}{k^{\prime \prime 2}} \frac{\partial}{\partial k^{\prime \prime}}+\frac{1}{k^{\prime \prime}} \frac{\partial^{2}}{\partial k^{\prime \prime 2}}\right)\left(\mathrm{i} \mathcal{P} \frac{1}{k^{2}-k^{\prime \prime 2}}+\pi \delta\left(k^{2}-k^{\prime \prime 2}\right)\right)
\end{aligned}
$$

and using this expression the second moment becomes a sum of three terms,

$$
\begin{aligned}
& \int_{0}^{\infty} \mathrm{d} \tau \tau^{2} C(\tau)=T_{1}+T_{2}+T_{3} \\
& =\frac{m^{2}}{4 \hbar^{2}} \int_{-\infty}^{\infty} \mathrm{d} k \mathrm{~d} k^{\prime \prime} \frac{\left(k^{\prime \prime 2}-k^{2}\right)^{2}}{k^{\prime \prime}|k|}|\widetilde{\psi}(k)|^{2}\left|\int_{x_{1}}^{x_{2}} \mathrm{~d} x \mathrm{e}^{\mathrm{i}\left(k^{\prime \prime}-k\right) x}\right|^{2} \\
& \times\left(-\frac{1}{k^{\prime \prime 2}} \frac{\partial}{\partial k^{\prime \prime}}+\frac{1}{k^{\prime \prime}} \frac{\partial^{2}}{\partial k^{\prime \prime 2}}\right) \delta\left(k^{2}-k^{\prime \prime 2}\right) \\
& +\frac{m^{2}}{4 \hbar^{2}} \int_{-\infty}^{\infty} \mathrm{d} k \mathrm{~d} k^{\prime \prime} \frac{\left(k^{\prime \prime 2}-k^{2}\right)^{2}}{k^{\prime \prime}|k|} \operatorname{Re}\left\{\widetilde{\psi}(k) \widetilde{\psi}(-k) \int_{x_{1}}^{x_{2}} \mathrm{~d} x \mathrm{~d} x^{\prime} \mathrm{e}^{\mathrm{i}\left(k^{\prime \prime}-k\right) x} \mathrm{e}^{-\mathrm{i}\left(k^{\prime \prime}+k\right) x^{\prime}}\right\} \\
& \times\left(-\frac{1}{k^{\prime \prime 2}} \frac{\partial}{\partial k^{\prime \prime}}+\frac{1}{k^{\prime \prime}} \frac{\partial^{2}}{\partial k^{\prime \prime 2}}\right) \delta\left(k^{2}-k^{\prime \prime 2}\right) \\
& -\frac{m^{2}}{4 \pi \hbar^{2}} \int_{-\infty}^{\infty} \mathrm{d} k \mathrm{~d} k^{\prime \prime} \frac{\left(k^{\prime \prime 2}-k^{2}\right)^{2}}{k^{\prime \prime}|k|} \operatorname{Im}\left\{\widetilde{\widetilde{\psi}(k)} \widetilde{\psi}(-k) \int_{x_{1}}^{x_{2}} \mathrm{~d} x \mathrm{~d} x^{\prime} \mathrm{e}^{\mathrm{i}\left(k^{\prime \prime}-k\right) x} \mathrm{e}^{-\mathrm{i}\left(k^{\prime \prime}+k\right) x^{\prime}}\right\} \\
& \times\left(-\frac{1}{k^{\prime \prime 2}} \frac{\partial}{\partial k^{\prime \prime}}+\frac{1}{k^{\prime \prime}} \frac{\partial^{2}}{\partial k^{\prime \prime 2}}\right) \frac{1}{k^{2}-k^{\prime \prime 2}} .
\end{aligned}
$$

The imaginary part of the term in brackets vanishes due to Eq. (4.12), thus $T_{3}=0$.

The first term can be evaluated with a partial integration,

$$
\begin{aligned}
T_{1}=\frac{m^{2}}{2 \hbar^{2}} \int_{-\infty}^{\infty} \mathrm{d} k \mathrm{~d} k^{\prime \prime} \frac{|\widetilde{\psi}(k)|^{2}}{|k|} \delta\left(k^{2}-k^{\prime \prime 2}\right) & \left(\frac{\partial}{\partial k^{\prime \prime}} \frac{1}{k^{\prime \prime}}+\frac{\partial^{2}}{\partial k^{\prime \prime 2}}\right) \\
& \times\left(\frac{\left(k+k^{\prime \prime}\right)^{2}}{k^{\prime \prime 2}}\left(1-\cos \left[\left(k^{\prime \prime}-k\right) L\right]\right)\right),
\end{aligned}
$$


and explicit derivation and integration over the delta function leads to

$$
T_{1}=\int_{-\infty}^{\infty} \mathrm{d} k \frac{m^{2} L^{2}}{\hbar^{2}|k|^{2}}\left(1+\frac{\sin ^{2}(k L)}{k^{2} L^{2}}\right)|\widetilde{\psi}(k)|^{2} .
$$

The proceeding for the second term is similar, after the $x$-integrations and partial integration with respect to $k^{\prime \prime}$ one has

$$
\begin{array}{r}
T_{2}=\frac{m^{2}}{2 \hbar^{2}} \int_{-\infty}^{\infty} \mathrm{d} k \mathrm{~d} k^{\prime \prime} \frac{\overline{\widetilde{\psi}(k)} \widetilde{\psi}(-k)}{|k|} \mathrm{e}^{-\mathrm{i} k\left(x_{1}+x_{2}\right)} \delta\left(k^{2}-k^{\prime \prime 2}\right)\left(\frac{\partial}{\partial k^{\prime \prime}} \frac{1}{k^{\prime \prime}}+\frac{\partial^{2}}{\partial k^{\prime \prime 2}}\right) \\
\times\left(\frac{k^{\prime \prime 2}-k^{2}}{k^{\prime \prime 3}}\left(\cos (k L)-\cos \left(k^{\prime \prime} L\right)\right)\right),
\end{array}
$$

and again, explicit derivation and integration over the delta function yields

$$
T_{2}=\int_{-\infty}^{\infty} \mathrm{d} k \frac{m^{2} L^{2}}{\hbar^{2}|k|^{2}} \frac{2 \sin (k L) \mathrm{e}^{-\mathrm{i} k\left(x_{1}+x_{2}\right)}}{k L} \widetilde{\psi}(k) \widetilde{\psi}(-k) .
$$

The final result for the second moment is the sum of $T_{1}$ and $T_{2}$, i.e. one has

$$
\begin{aligned}
& \int_{0}^{\infty} \mathrm{d} \tau \tau^{2} C(\tau) \\
& \quad=\int_{-\infty}^{\infty} \mathrm{d} k \frac{m^{2} L^{2}}{\hbar^{2}|k|^{2}}\left[|\widetilde{\psi}(k)|^{2}\left(1+\frac{\sin ^{2}(k L)}{k^{2} L^{2}}\right)+\overline{\widetilde{\psi}(k)} \widetilde{\psi}(-k) \frac{2 \sin (k L) \mathrm{e}^{-\mathrm{i} k\left(x_{1}+x_{2}\right)}}{k L}\right] .
\end{aligned}
$$

Remarkably, this expression coincides with the second moment of the ideal dwelltime distribution $\Pi_{\mathrm{D}}(t)$, given in Eq. (2.76).

To conclude, it has been shown in this section that the first and the second moment of a quantum flux-flux correlation function, defined in Eq. (4.6), coincide with the corresponding moments of the dwell-time operator $\widehat{T}_{\mathrm{D}}$,

$$
\begin{aligned}
\left\langle\psi\left|\int_{0}^{\infty} \mathrm{d} \tau \tau \int_{-\infty}^{\infty} \mathrm{d} t \operatorname{Re} \widehat{C}\left(x_{1}, x_{2} ; t, \tau\right)\right| \psi\right\rangle & =\left\langle\psi\left|\widehat{T}_{\mathrm{D}}\right| \psi\right\rangle, \\
\left\langle\psi\left|\int_{0}^{\infty} \mathrm{d} \tau \tau^{2} \int_{-\infty}^{\infty} \mathrm{d} t \operatorname{Re} \widehat{C}\left(x_{1}, x_{2} ; t, \tau\right)\right| \psi\right\rangle & =\left\langle\psi\left|\widehat{T}_{\mathrm{D}}^{2}\right| \psi\right\rangle .
\end{aligned}
$$

Since $\psi$ is arbitrary, the two operators on the left hand side and the right hand side of Eq. (4.24) coincide. Moreover, the result for the second moment suggests that the flux-flux correlation function $C(\tau)$ can be understood as a dwell-time distribution with similar properties as $\Pi_{\mathrm{D}}(t)$, although $C(\tau)$ is not necessarily positive. To what extent these two distributions agree has to be shown in future research. For an operational understanding of dwell-time distributions the correspondence to fluxflux correlations seems to be advantageous, since the quantum mechanical flux itself has been shown to be measurable in Section 3.2.3. 


\subsection{Operational approach to flux-flux correlation functions}

It has been shown in Section 3.2 that by means of physical intuition the ideal time-ofarrival distribution of a particle, for instance the flux, can be modeled by a quantity which is a priori not related to this ideal quantity, but which can "measure" a similar property of the physical situation. For arrival times, this operational quantity is the first-photon distribution.

The previous section showed the close connection between dwell-time distributions and flux-flux correlation functions. For an operational model to dwell times, one has to find a quantity which approximately "measures" a flux-flux correlation. According to the connection between the flux and a first-photon distribution, a first guess to such a quantity might be a photon-photon correlation function.

In the following, a first derivation that is based on this idea is presented and some results concerning a possible photon-photon correlation function of moving atoms are obtained. It turns out that the model is not appropriate to describe dwell-time distributions at this stage, thus this section can actually be understood as an outlook for further research.

\subsubsection{Photon-photon correlations}

The probability density for the detection of a photon at time $t$ when the initial state at time $t=0$ is given by $\psi_{0}$ has been obtained in Eq. (3.18). It reads

$$
I\left(t ; \psi_{0}\right)=w_{1}\left(t ; \psi_{0}\right)+\int_{0}^{t} \mathrm{~d} t^{\prime} I\left(t^{\prime} ; \psi_{0}\right) w_{1}\left(t-t^{\prime} ; \psi_{t^{\prime}}^{\mathscr{R}}\right) .
$$

For the probability density of an emission at time $t$ and another emission at time $t+\tau$ it follows that

$$
G(t, t+\tau)=I\left(t ; \psi_{0}\right) I\left(t+\tau ; \psi_{0}\right) .
$$

The integral equation (4.26) is hard to solve since the reset state generally depends on the time $t^{\prime}$. Therefore in the following only first-photon-first-photon correlations are considered, that means temporal correlations between the first and the second detection. The relation to flux-flux correlations is given by the following idealized setup: Consider a two-level atom which is initially in the ground state and comes in from the far left. For convenience, the spatial region for which the dwell-time distribution is to be measured is assumed to be located between $x_{1}=0$ and $x_{2}=L$. Firstly, the laser field is located in the right half-space, $x \geq 0$. The corresponding conditional Hamiltonian is given with Eq. (3.55) by

$$
H_{\mathrm{c}}^{(1)}=\frac{\widehat{p}^{2}}{2 m}+\frac{\hbar}{2}\left(\begin{array}{cc}
0 & \Omega \Theta(\widehat{x}) \\
\Omega \Theta(\widehat{x}) & -\mathrm{i} \gamma
\end{array}\right) .
$$

The temporal probability density for the emission of the first photon is given by $w_{1}\left(t ; \psi_{0}\right)=-\frac{\mathrm{d}}{\mathrm{d} t} \| \exp \left(-\mathrm{i} H_{\mathrm{c}}^{(1)} t / \hbar\right)\left|\psi_{0}\right\rangle|1\rangle \|^{2}$. Right after the emission of the first photon, which is assumed to take place at time $t$, the laser field between $x=0$ and 
$x=L$ is switched off, such that the unnormalized reset state after the emission at time $t$, which reads with Eq. (3.154)

$$
\begin{aligned}
\left|\psi_{t}^{\mathscr{R}}\right\rangle & =\sqrt{\gamma}|1\rangle\left\langle 2\left|\mathrm{e}^{-\mathrm{i} H_{c}^{(1)} t / \hbar}\right| \psi_{0}\right\rangle|1\rangle \\
& =\sqrt{\gamma} \int_{0}^{\infty} \mathrm{d} k \widetilde{\psi}(k) \mathrm{e}^{-\mathrm{i} \hbar k^{2} t / 2 m}\left|\phi_{k}^{(2)}\right\rangle|1\rangle,
\end{aligned}
$$

evolves further with the conditional Hamiltonian

$$
H_{\mathrm{c}}^{(2)}=\frac{\widehat{p}^{2}}{2 m}+\frac{\hbar}{2}\left(\begin{array}{cc}
0 & \Omega \Theta(\widehat{x}-L) \\
\Omega \Theta(\widehat{x}-L) & -\mathrm{i} \gamma
\end{array}\right) .
$$

In Eq. (4.29), $\left|\phi_{k}^{(2)}\right\rangle$ is given by Eq. (3.57). The probability density for the first emission after the resetting is $w_{1}\left(\tau ; \psi_{t}^{\mathscr{R}} /\left\|\psi_{t}^{\mathscr{R}}\right\|\right)=-\frac{\mathrm{d}}{\mathrm{d} \tau} \| \exp \left(-\mathrm{i} H_{\mathrm{c}}^{(2)} \tau / \hbar\right)\left|\psi_{t}^{\mathscr{R}}\right\rangle /\left(\left\|\psi_{t}^{\mathscr{R}}\right\|\right) \|^{2}$. The experiment is aborted after two photon emissions. In this case, Eq. (4.27) becomes

$$
G(t, t+\tau)=w_{1}\left(t ; \psi_{0}\right) w_{1}\left(\tau ; \psi_{t}^{\mathscr{R}} /\left\|\psi_{t}^{\mathscr{R}}\right\|\right),
$$

where the squared norm of the reset state is given with Eqs. (3.45) and (3.70) by

$$
\left\|\psi_{t}^{\mathscr{R}}\right\|^{2}=\gamma \int_{-\infty}^{\infty} \mathrm{d} x\left|\psi_{t}^{(2)}(x)\right|^{2}=w_{1}\left(t ; \psi_{0}\right)
$$

and thus

$$
G(t, t+\tau)=w_{1}\left(\tau ; \psi_{t}^{\mathscr{R}}\right) .
$$

As for the flux-flux correlation function, this expression has to be integrated over $t$ to sum up all contributions of correlations irrespectively of the time of occurrence for the first detection. This leads to a photon-photon correlation function of the following form:

$$
G(\tau)=\int_{-\infty}^{\infty} \mathrm{d} t G(t, t+\tau)=\int_{-\infty}^{\infty} \mathrm{d} t w_{1}\left(\tau ; \psi_{t}^{\mathscr{Q}}\right) .
$$

The difficulties with this expression come from the fact that $\psi_{t}^{\mathscr{R}}$ has to be developed with $H_{\mathrm{c}}^{(2)}$, i.e. it has to be expanded in the eigenbasis of $H_{\mathrm{c}}^{(2)}$. For this derivation, further research is necessary. As an additional simplification, one might consider the regime of negligible delay and reflection given by Eq. (3.83), since in this case the first photon distribution $w_{1}\left(\tau ; \psi_{t}^{\mathscr{R}}\right)$ can be approximated by the flux of the freely evolved reset state at $x=L$. A similar treatment has been investigated in Ref. [115] for absorbing potentials. However, complications arise with this approach as well, since the regime of negligible delay and reflection relies on the presence of a smallest significant energy component in the wave packet, but $\psi_{t}^{\mathscr{R}}$ has different significant momentum contributions depending on the time $t$ of the reset event.

Thus, reasonable as the operational modeling of flux-flux correlations by means of photon-photon correlation functions may be, a confirmation of this relation remains as a challenge for further research. 


\section{Chapter 5}

\section{Operator normalization}

\subsection{Operator normalization of the quantum opti- cal arrival-time model}

Recently, Brunetti and Fredenhagen [27] proposed a general construction of an observable measuring the "time of occurrence" of some event. This construction involved an unitary time development and a normalization procedure on the level of operators, not on the level of expectations values as introduced in Eq. (3.93). For this purpose they constructed a positive operator on the orthogonal complement of the states on which the time of occurrence is infinite or zero and used its square root for normalization.

In this section it will be shown that normalization on the level of operators can also be applied to the laser-based arrival-time model given in Section 3.2. This has been suggested in Ref. [24]. The advantage of normalizing the first-photon distribution on the level of operators is, that this procedure preserves its property of a bilinear form of the state $\psi$. As a central result, the operator-normalized distribution in the limit of vanishing delay turns out to be equal to Kijowski's arrivaltime distribution $\Pi_{K}(t)$ [84]. This allows a generalization of Kijowski's distribution in the presence of external potentials which will be given in Chapter 6 .

\subsubsection{Normalization on the level of operators}

To employ the operator normalization one has to rewrite Eq. (3.68) in operator form. The two-channel Hamiltonian for the conditional time development, $H_{\mathrm{c}}$, is given by Eq. (3.55). For a concise and simplified notation, it is convenient to go to the interaction picture with respect to $H_{0}=\widehat{p}^{2} / 2 m$,

$$
\begin{aligned}
H_{\mathrm{c}}^{\mathrm{I}} & =\mathrm{e}^{\mathrm{i} H_{0} t / \hbar}\left(H_{\mathrm{c}}-H_{0}\right) \mathrm{e}^{-\mathrm{i} H_{0} t / \hbar} \\
U_{\mathrm{c}}^{\mathrm{I}}\left(t, t_{0}\right) & =\mathrm{e}^{\mathrm{i} H_{0} t / \hbar} \mathrm{e}^{-\mathrm{i} H_{\mathrm{c}}\left(t-t_{0}\right) / \hbar} \mathrm{e}^{-\mathrm{i} H_{0} t_{0} / \hbar},
\end{aligned}
$$

where $U_{\mathrm{c}}^{\mathrm{I}}$ is the conditional time development corresponding to $H_{\mathrm{c}}^{\mathrm{I}}$. Let $|\psi\rangle|1\rangle$ be the wave packet that corresponds to the form of the incoming ground state at $t=0$ 
if it would freely evolve in the absence of the laser field. Then Eq. (3.66) can be written as

$$
\left|\boldsymbol{\Psi}_{t}\right\rangle=\mathrm{e}^{-\mathrm{i} H_{0} t / \hbar} U_{\mathrm{c}}^{\mathrm{I}}(t,-\infty)|\psi\rangle|1\rangle
$$

and Eq. (3.67) as

$$
N(t)=\left\langle 1\left|\left\langle\psi\left|\widehat{N}_{t}\right| \psi\right\rangle\right| 1\right\rangle,
$$

where $\widehat{N}_{t}$ is a time-dependent operator in the Heisenberg picture,

$$
\widehat{N}_{t}=U_{\mathrm{c}}^{\mathrm{I}}(t,-\infty)^{\dagger} U_{\mathrm{c}}^{\mathrm{I}}(t,-\infty) .
$$

For the first-photon distribution it follows that

$$
\Pi(t)=\left\langle 1\left|\left\langle\psi\left|\widehat{\Pi}_{t}\right| \psi\right\rangle\right| 1\right\rangle,
$$

and since $H_{\mathrm{c}}-H_{\mathrm{c}}^{\dagger}=-\mathrm{i} \hbar \gamma|2\rangle\langle 2|$ one has

$$
\begin{aligned}
\widehat{\Pi}_{t} & =-\frac{\mathrm{d} \widehat{N}(t)}{\mathrm{d} t} \\
& =\gamma U_{\mathrm{c}}^{\mathrm{I}}(t,-\infty)^{\dagger}|2\rangle\langle 2| U_{\mathrm{c}}^{\mathrm{I}}(t,-\infty) .
\end{aligned}
$$

In analogy to Eq. (3.71), the integral

$$
\begin{aligned}
\int_{-\infty}^{\infty} \mathrm{d} t \widehat{\Pi}_{t} & =\widehat{N}_{-\infty}-\widehat{N}_{\infty} \\
& =\widehat{1}-\widehat{N}_{\infty}
\end{aligned}
$$

is considered and the operator $\widehat{B}$ is defined on the incoming states (with internal ground state) through its matrix elements as

$$
\langle 1|\langle\varphi|\widehat{B}| \psi\rangle| 1\rangle=\left\langle 1\left|\left\langle\varphi\left|\widehat{1}-\widehat{N}_{\infty}\right| \psi\right\rangle\right| 1\right\rangle .
$$

The operator $\widehat{B}$ can be calculated as follows. From Eq. (3.72) one sees that for large $t$ the second component of $\boldsymbol{\Psi}(x, t)$ is damped away and therefore only the reflected wave remains. Pulling $\mathrm{e}^{-\mathrm{i} \hbar k^{2} t / 2 m}$ out from the integral as $\mathrm{e}^{-\mathrm{i} H_{0} t / \hbar}$ one concludes, from Eqs. (3.72) and (5.2), that

$$
U_{\mathrm{c}}^{\mathrm{I}}(\infty,-\infty)|\psi\rangle|1\rangle=\int_{0}^{\infty} \mathrm{d} k \widetilde{\psi}(k) R_{1}(k)|-k\rangle|1\rangle .
$$

Taking the scalar product with $U_{\mathrm{c}}^{\mathrm{I}}(\infty,-\infty)|\varphi\rangle|1\rangle$ one finds from Eq. (5.4),

$$
\left\langle 1\left|\left\langle\varphi\left|\widehat{N}_{\infty}\right| \psi\right\rangle\right| 1\right\rangle=\int_{0}^{\infty} \mathrm{d} k \overline{\widetilde{\varphi}(k)} \widetilde{\psi}\left(k^{\prime}\right) \overline{R_{1}(k)} R_{1}\left(k^{\prime}\right) \delta\left(k-k^{\prime}\right) .
$$

With Eq. (5.9) and in $k$-space it follows that

$$
\left\langle 1\left|\left\langle k|\widehat{B}| k^{\prime}\right\rangle\right| 1\right\rangle=\left(1-\overline{R_{1}(k)} R_{1}\left(k^{\prime}\right)\right) \delta\left(k-k^{\prime}\right) .
$$


Hence, on the incoming states, one can define the positive operator

$$
\widehat{\Pi}_{t}^{\mathrm{ON}}=\widehat{B}^{-1 / 2} \widehat{\Pi}_{t} \widehat{B}^{-1 / 2}
$$

Since $\left|R_{1}(k)\right|<1, \widehat{B}$ is not only a positive operator but also its inverse square-root exists. From Eqs. (5.9) and (5.6) it is obvious that $\int_{-\infty}^{\infty} \mathrm{d} t \widehat{\Pi}_{t}^{\mathrm{ON}}=\widehat{1}$ and so the probability distribution

$$
\Pi^{\mathrm{ON}}(t)=\left\langle 1\left|\left\langle\psi\left|\widehat{\Pi}_{t}^{\mathrm{ON}}\right| \psi\right\rangle\right| 1\right\rangle
$$

is normalized to 1 . This procedure is referred to as operator normalization in the following. From Eqs. (5.13) and (5.8) one finally obtains

$$
\begin{gathered}
\Pi^{\mathrm{ON}}(t)=\gamma \int_{-\infty}^{\infty} \mathrm{d} x \int \mathrm{d} k \mathrm{~d} k^{\prime} \overline{\widetilde{\psi}(k)} \widetilde{\psi}\left(k^{\prime}\right)\left(1-\left|R_{1}(k)\right|^{2}\right)^{-1 / 2}\left(1-\left|R_{1}\left(k^{\prime}\right)\right|^{2}\right)^{-1 / 2} \\
\times \mathrm{e}^{\mathrm{i} \hbar\left(k^{2}-k^{\prime 2}\right) t / 2 m} \overline{\phi_{k}^{(2)}(x)} \phi_{k^{\prime}}^{(2)}(x) .
\end{gathered}
$$

Operator normalization can be viewed as a change in the incident momentum distribution $\widetilde{\psi}(k)$ by a factor of $\left(1-\left|R_{1}(k)\right|^{2}\right)^{-1 / 2}$, as shown for a Gaussian wave packet in Fig. 5.1. For mean initial velocities of the order of $\mathrm{cm} / \mathrm{s}$ a single wave packet is multiplied by a nearly constant factor. Only for very slow particles and $\Omega \gg \gamma$ a distortion of the packet occurs. The slow momentum components are enhanced by operator normalization, since they are preferably reflected. This leads to an additional delay of $\Pi^{\mathrm{ON}}(t)$ compared to $\Pi(t)$.

In Section 5.4 it will be shown that the appearance of the additional factor $\left(1-\left|R_{1}(k)\right|^{2}\right)^{-1 / 2}$ can be seen as a filtering procedure which has to be applied to the incoming wave packet in order to obtain the same result as for an operator normalization.

\subsubsection{Connection to Kijowski's arrival-time distribution}

In the previous section it has been shown that a specific normalization procedure can be applied to the first-photon distribution without loosing the property of a bilinear form. By this approach one eliminates the consequences of the reflection problem. To eliminate simultaneously the delay problem, the limit of Eq. (3.81) has to be applied, $\gamma \rightarrow \infty$ with $\gamma / \Omega=$ const. This limit has been already derived in Section 3.2.4. With Eq. (3.98) one has

$$
\left(1-\left|R_{1}(k)\right|^{2}\right)^{-1 / 2}\left(1-\left|R_{1}\left(k^{\prime}\right)\right|^{2}\right)^{-1 / 2} \sim \frac{1}{4 c_{1}(\alpha)} \sqrt{\frac{2 m \gamma}{\hbar k k^{\prime}}}
$$




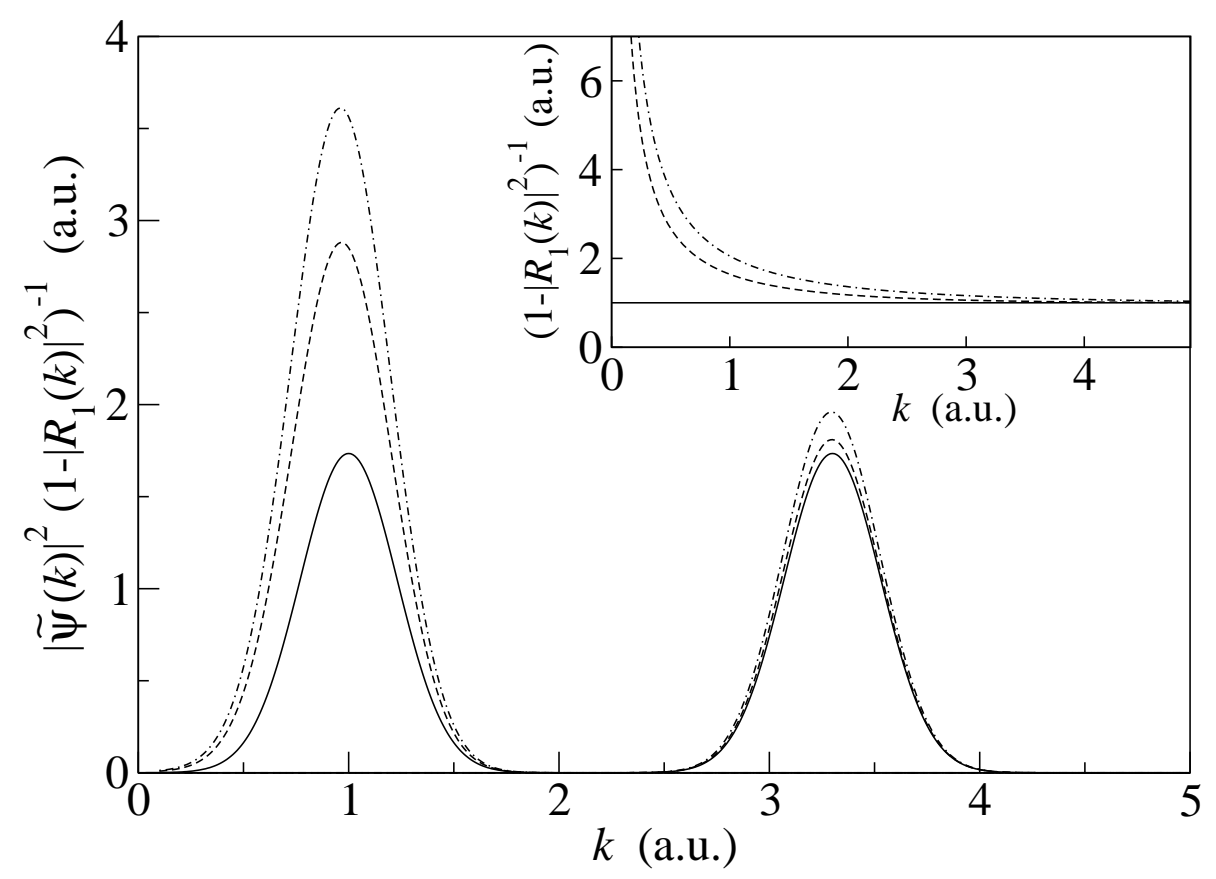

Figure 5.1: Operator normalization viewed as change of the initial momentum distribution. Two Gaussian momentum wave packets with $\langle v\rangle_{1}=1.0,\langle v\rangle_{2}=3.3$, $\Delta v_{1}=\Delta v_{2}=0.23$, without (solid line) and with operator normalization for $\Omega=0.66 \gamma_{\mathrm{Cs}}$ (dashed line) and $\Omega=\gamma_{\mathrm{Cs}}$ (dot-dashed line).

and inserting this result together with Eq. (3.103) into Eq. (5.15) yields in leading order

$$
\begin{aligned}
\Pi^{\mathrm{ON}}(t) \sim & \frac{\hbar}{2 \pi m} \int \mathrm{d} k \mathrm{~d} k^{\prime} \overline{\widetilde{\psi}(k)} \widetilde{\psi}\left(k^{\prime}\right) \mathrm{e}^{\mathrm{i} \hbar\left(k^{2}-k^{\prime 2}\right) t / 2 m} \sqrt{k k^{\prime}} \\
& \times \frac{1}{4 c_{1}(\alpha)} \sqrt{\frac{2 m \gamma}{\hbar}} \int_{-\infty}^{\infty} d x\left\{\Theta(-x) c_{2}^{2}(\alpha) \mathrm{e}^{-\mathrm{i}(q-\bar{q}) x}\right. \\
& \left.\quad+\Theta(x) \frac{16}{c_{3}^{2}(\alpha)} \frac{\Omega^{2}}{\gamma^{2}}\left|\left(1+\sqrt{\frac{1+\alpha}{2}}\right) \mathrm{e}^{\mathrm{i} k_{+} x}-\left(1+\sqrt{\frac{1-\alpha}{2}}\right) \mathrm{e}^{\mathrm{i} k_{-} x}\right|^{2}\right\}
\end{aligned}
$$

The expression after $\times$ is independent of $k$ and $k^{\prime}$, since in the above limit $q$ and $k_{ \pm}$are given by Eqs. (3.96) and (3.97). One can insert the $c_{i}(\alpha)$ from Eqs. (3.100)(3.102) and explicitly calculate the integral over $x$, but it is easier to note that the term before $\times$ is just Kijowski's distribution, which is normalized to 1, and therefore the expression after $\times$ has to be equal to 1 . Thus, one obtains

$$
\Pi^{\mathrm{ON}}(t) \rightarrow \Pi_{\mathrm{K}}(t), \quad \gamma \rightarrow \infty, \gamma / \Omega=\text { const. }
$$

This remarkable result provides for the first time a connection between the ideal arrival-time distribution of Kijowski and the operational first-photon distribution. 
Operator normalization compensates for reflection losses in a way that for strong driving the distribution is normalized, too. Therefore the slow momentum components have to be enhanced, as it is shown in Fig. 5.1. To investigate the quality of the approximation for large but finite $\gamma$, the operator-normalized distribution $\Pi^{\mathrm{ON}}(t)$ is plotted in Fig. 5.2 for the same example as in Fig. 3.5, where the parameters are chosen in a way that the difference between $\Pi_{K}(t)$ and $J(t)$ is enhanced. One clearly recognizes that for a large decay rate and a large laser intensity $\Pi^{\mathrm{ON}}(t)$ is in good agreement with $\Pi_{K}(t)$.

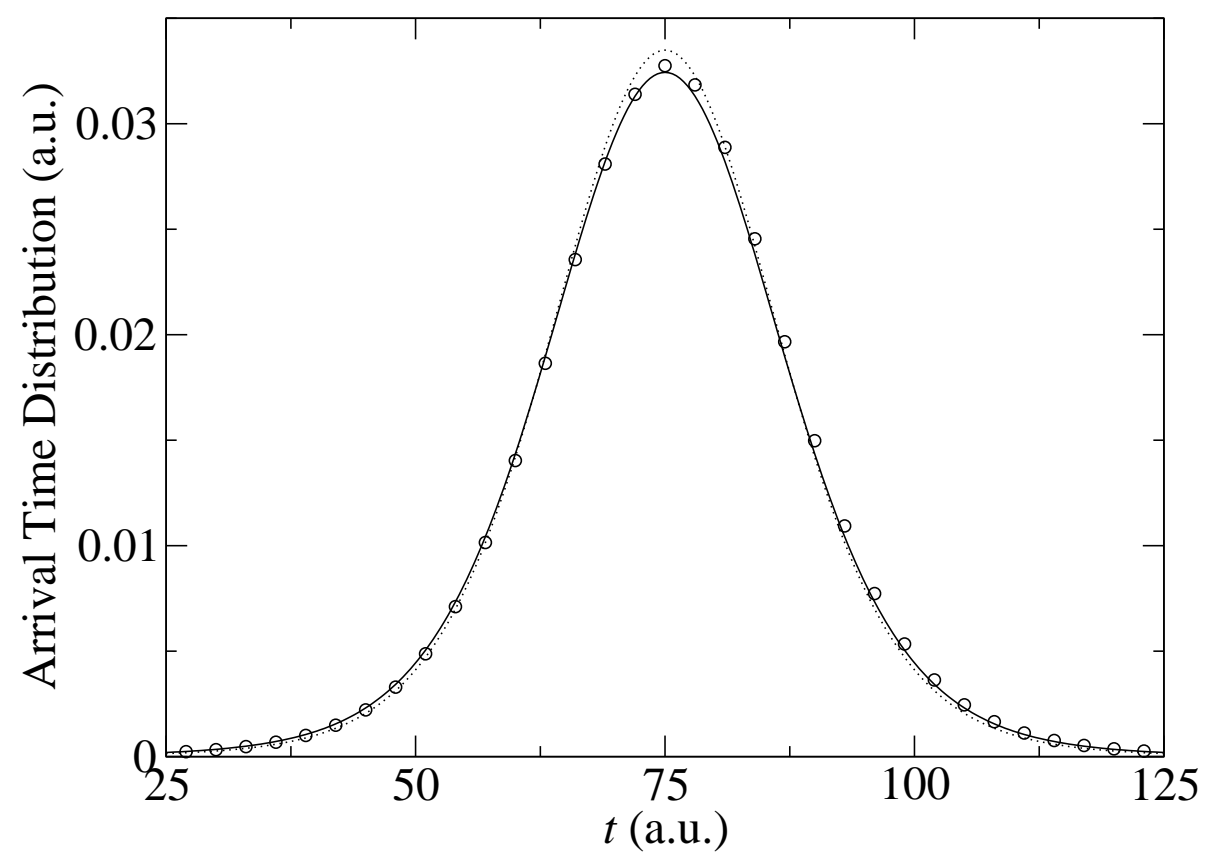

Figure 5.2: Good agreement of the operator-normalized distribution $\Pi^{\mathrm{ON}}(t)$ (circles) with Kijowski's distribution $\Pi_{\mathrm{K}}(t)$ (solid line) for large but finite $\gamma=10 \gamma_{\mathrm{Cs}}$ and $\Omega=0.33 \gamma$. The other parameters are the same as in Fig. 3.5. The significant difference to the flux (dotted line) is obvious.

\subsubsection{Fixed decay rate}

The fact that the decay rate $\gamma$ is in general not adjustable is a disadvantage of the limit presented in the previous section. Therefore in this section the limit of large driving, i.e. $\Omega \rightarrow \infty$ with $\gamma=$ const. is considered. Experimentally, $\Omega \rightarrow \infty$ is easy to achieve by enhancing the laser intensity. Clearly, this will not eliminate the delay problem, since the mean waiting time for the first photon and for an atom at rest approaches $t_{W} \rightarrow 2 / \gamma$ for $\Omega \rightarrow \infty$ as it can be seen from Eq. (3.78). Therefore, it will be shown that an ideal distribution can be obtained by means of a deconvolution, similar to the procedure given in Section 3.2.3. Again, this ideal distribution turns out to be asymptotically equivalent to Kijowski's distribution.

The limit $\Omega \rightarrow \infty$ with $\gamma$ held fixed has already been considered in Section 3.5.3. Results for this limit are given in Eqs. (3.182)-(3.186) and in Eq. (3.187) and it 
follows that

$$
\left(1-\left|R_{1}(k)\right|^{2}\right)^{-\frac{1}{2}}\left(1-\left|R_{1}\left(k^{\prime}\right)\right|^{2}\right)^{-\frac{1}{2}} \sim \frac{1}{2} \sqrt{\frac{m \Omega}{\hbar k k^{\prime}}} .
$$

When integrating Eq. (3.187) over $x$, only the term $\mathrm{e}^{-\mathrm{i}\left(\bar{k}_{+}-k_{+}^{\prime}\right) x}$ contributes in leading order of $\Omega$, and inserting this expression together with Eq. (5.19) into the operator normalized first-photon distribution (5.15) leads to

$$
\Pi^{\mathrm{ON}}(t) \rightarrow \frac{\hbar}{2 \pi m} \int \mathrm{d} k \mathrm{~d} k^{\prime} \widetilde{\psi}(k) \widetilde{\psi}\left(k^{\prime}\right) \mathrm{e}^{\mathrm{i} \frac{\hbar}{2 m}\left(k^{2}-k^{\prime 2}\right) t} \frac{\gamma \sqrt{k k^{\prime}}}{\gamma+\mathrm{i} \hbar\left(k^{2}-k^{\prime 2}\right) / m} .
$$

For $\gamma \rightarrow \infty$ one again directly obtains Kijowski's distribution, but for finite $\gamma$ one has a delay. It can be eliminated, as shown in Section 3.2.3, by a deconvolution with the first-photon distribution $W(t)$ of an atom at rest in the limit $\Omega \rightarrow \infty$, making the ansatz

$$
\Pi^{\mathrm{ON}}(t)=\left(\Pi_{\mathrm{id}}^{\mathrm{ON}} * W\right)(t)
$$

for an ideal distribution $\Pi_{\mathrm{id}}(t)$. In terms of Fourier transforms one has

$$
\widetilde{\Pi}_{\mathrm{id}}^{\mathrm{ON}}(\nu)=\frac{\widetilde{\Pi}^{\mathrm{ON}}(\nu)}{\widetilde{W}(\nu)},
$$

where $1 / \widetilde{W}(\nu)$ is given in Eq. (3.87) and it tends for $\Omega \rightarrow \infty$ to

$$
1 / \widetilde{W}(\nu) \sim 1+2 \mathrm{i} \nu / \gamma, \quad \Omega \rightarrow \infty,
$$

whereas $\widetilde{\Pi}^{\mathrm{ON}}(\nu)$ is given with Eq. (5.20) by

$$
\widetilde{\Pi}^{\mathrm{ON}}(t) \sim \frac{\hbar}{2 \pi m} \int \mathrm{d} k \mathrm{~d} k^{\prime} \overline{\widetilde{\psi}(k)} \widetilde{\psi}\left(k^{\prime}\right) 2 \pi \delta\left(\nu-\frac{\hbar}{2 m}\left(k^{2}-k^{\prime 2}\right)\right) \frac{\gamma \sqrt{k k^{\prime}}}{\gamma+\frac{\mathrm{i} \hbar}{m}\left(k^{2}-k^{\prime 2}\right)} .
$$

Inserting these two expressions into Eq. (5.22) yields

$$
\widetilde{\Pi}_{\mathrm{id}}^{\mathrm{ON}}(\nu)=\frac{\hbar}{2 \pi m} \int \mathrm{d} k \mathrm{~d} k^{\prime} \overline{\widetilde{\psi}(k)} \widetilde{\psi}\left(k^{\prime}\right) \sqrt{k k^{\prime}} 2 \pi \delta\left(\nu-\frac{\hbar}{2 m}\left(k^{2}-k^{\prime 2}\right)\right),
$$

and in the time domain one finally has

$$
\Pi_{\mathrm{id}}^{\mathrm{ON}}(t) \rightarrow \Pi_{\mathrm{K}}(t), \quad \Omega \rightarrow \infty .
$$

Thus, the deconvolution leads to a positive arrival time distribution. This result shows that also for atoms with a fixed decay rate $\gamma$ Kijowski's distribution can be reconstructed from the operator normalized first-photon distribution by considering strong driving and by deconvoluting the data with the distribution $W(t)$. An example for this approach is shown in Fig. 5.3, for similar parameter values as in Fig. 3.4. The deconvoluted $\Pi_{\mathrm{id}}^{\mathrm{ON}}(t)$ perfectly agrees with $\Pi_{\mathrm{K}}(t)$ and it differs noticeable from the flux.

The only drawback in this procedure is the fact that so far no operational approach to the operator normalization has been given. This problem is solved in Section 5.4 by considering the operator normalization as a (pre-)filtering of the incoming wave packet. 


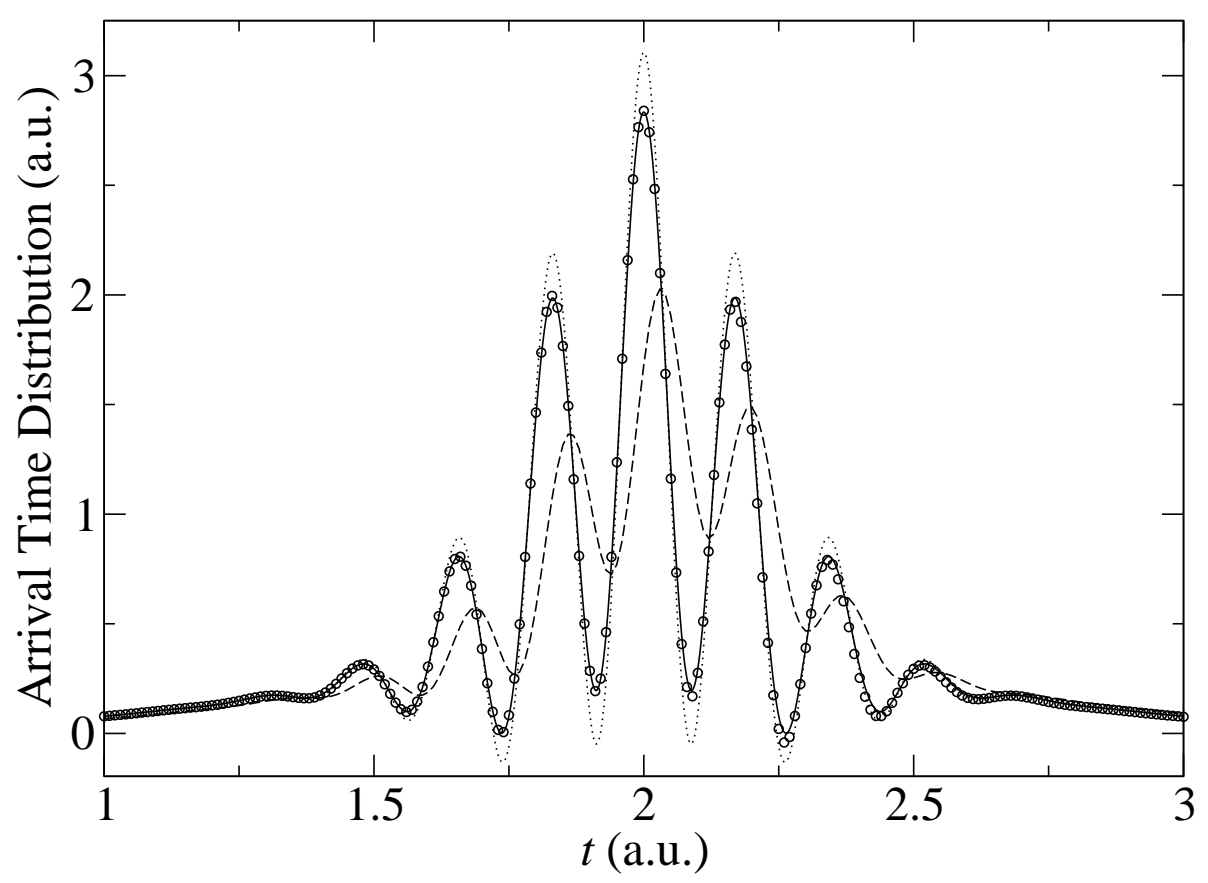

Figure 5.3: Excellent agreement between the deconvoluted operator-normalized distribution $\Pi_{\mathrm{id}}^{\mathrm{ON}}(t)$ (white circles) and $\Pi_{\mathrm{K}}(t)$ (solid line) for large $\Omega=500 \gamma_{\mathrm{Cs}}$. Shown is also $\Pi^{\mathrm{ON}}(t)$ before deconvolution (dashed line). The initial wave packet is a coherent combination $\psi=\left(\psi_{1}+\psi_{2}\right) / \sqrt{2}$ of two Gaussian states for the center-of-mass motion of a single cesium atom that become separately minimum-uncertainty packets (with $\Delta x_{1}=\Delta x_{2}=1.45$ and average velocities $\langle v\rangle_{1}=8.90,\langle v\rangle_{2}=2.54$ ) at $x=0$ and $t=2$. The flux (dotted) becomes negative for some $t$.

\subsubsection{Operator normalization and connection with the flux}

Instead of the operator-normalized expression of Eq. (5.13) one may also consider the expectation value of the not manifestly positive expression

$$
\widehat{\Pi}_{t}^{J}=\frac{1}{2}\left(\widehat{B}^{-1} \widehat{\Pi}_{t}+\widehat{\Pi}_{t} \widehat{B}^{-1}\right)
$$

with $\widehat{B}$ and $\widehat{\Pi}_{t}$ given in Eqs. (5.12) and (5.6), respectively. Clearly, the distribution

$$
\Pi^{J}(t)=\left\langle 1\left|\left\langle\psi\left|\widehat{\Pi}_{t}^{J}\right| \psi\right\rangle\right| 1\right\rangle
$$

is also normalized to 1 . In $k$-space one has in analogy to Eq. (5.15)

$$
\begin{aligned}
\Pi^{J}(t)=\gamma \int_{-\infty}^{\infty} \mathrm{d} x \int \mathrm{d} k \mathrm{~d} k^{\prime} \overline{\widetilde{\psi}(k)} \widetilde{\psi}\left(k^{\prime}\right) \frac{1}{2}\left(\frac{1}{1-\left|R_{1}(k)\right|^{2}}+\frac{1}{1-\left|R_{1}\left(k^{\prime}\right)\right|^{2}}\right) \\
\times \mathrm{e}^{\mathrm{i} \hbar\left(k^{2}-k^{\prime 2}\right) t / 2 m} \overline{\phi_{k}^{(2)}(x)} \phi_{k^{\prime}}^{(2)}(x) .
\end{aligned}
$$


Applying the limit of vanishing delay, $\gamma \rightarrow \infty, \gamma / \Omega=$ const., to this expression results with Eqs. (3.103) and (3.98) in

$$
\begin{aligned}
\Pi^{J}(t) & \rightarrow \frac{\hbar}{2 \pi m} \int \mathrm{d} k \mathrm{~d} k^{\prime} \overline{\widetilde{\psi}(k)} \widetilde{\psi}\left(k^{\prime}\right) \mathrm{e}^{\mathrm{i} \hbar\left(k^{2}-k^{\prime 2}\right) t / 2 m} \frac{k+k^{\prime}}{2} \\
& =J(t, 0),
\end{aligned}
$$

i.e. to the quantum mechanical flux. Note that the particular form of the operatornormalized operators, either Eq. (5.13) or Eq. (5.27), mirrors the corresponding symmetrization of the local velocity density.

\subsubsection{Alternative derivation of the operator normalization}

Once knowing that a normalization operator $\widehat{B}$ exists in the sense of Section 5.1, one can derive its particular form in a more convenient way. With Eq. (3.70) it follows that

$$
\begin{aligned}
\Pi(t) & =\gamma \int_{0}^{\infty} \mathrm{d} k \int_{0}^{\infty} \mathrm{d} k^{\prime} \overline{\widetilde{\psi}(k)} \widetilde{\psi}\left(k^{\prime}\right) \mathrm{e}^{\mathrm{i} \hbar\left(k^{2}-k^{\prime 2}\right) t / 2 m} \int_{-\infty}^{\infty} \mathrm{d} x \overline{\phi_{k}^{(2)}(x)} \phi_{k^{\prime}}^{(2)}(x) \\
& =\int_{0}^{\infty} \mathrm{d} k \int_{0}^{\infty} \mathrm{d} k^{\prime} \overline{\widetilde{\psi}(k)} \widetilde{\psi}\left(k^{\prime}\right) \mathrm{e}^{\mathrm{i} \hbar\left(k^{2}-k^{\prime 2}\right) t / 2 m} f\left(k, k^{\prime}\right)
\end{aligned}
$$

where the kernel function

$$
f\left(k, k^{\prime}\right)=\gamma \int_{-\infty}^{\infty} \mathrm{d} x \overline{\phi_{k}^{(2)}(x)} \phi_{k^{\prime}}^{(2)}(x)
$$

has been defined. The normalization on the level of operators leads to an additional kernel function $b(k)$ in $k$-space, and in a symmetric form the normalized distribution reads

$$
\Pi^{\mathrm{ON}}(t)=\int_{0}^{\infty} \mathrm{d} k \int_{0}^{\infty} \mathrm{d} k^{\prime} \bar{\psi}(k) \widetilde{\psi}\left(k^{\prime}\right) \mathrm{e}^{\mathrm{i} \hbar\left(k^{2}-k^{\prime 2}\right) t / 2 m} b(k)^{-1 / 2} b\left(k^{\prime}\right)^{-1 / 2} f\left(k, k^{\prime}\right) .
$$

Here $b(k)^{-1 / 2}$ and $b\left(k^{\prime}\right)^{-1 / 2}$ have to cancel the factors which arise from integrating $\Pi(t)$ in Eq. (5.32) over $t$, namely $(2 \pi m / \hbar k) f(k, k)$. It is important here that the $k$-integration runs from 0 to $\infty$. Therefore one has

$$
b(k)^{-1 / 2}=\left(\frac{\hbar k}{2 \pi m f(k, k)}\right)^{1 / 2}
$$

and this yields with Eq. (5.34)

$$
\Pi^{\mathrm{ON}}(t)=\int_{0}^{\infty} \mathrm{d} k \int_{0}^{\infty} \mathrm{d} k^{\prime} \overline{\widetilde{\psi}(k)} \widetilde{\psi}\left(k^{\prime}\right) \mathrm{e}^{\mathrm{i} \hbar\left(k^{2}-k^{\prime 2}\right) t / 2 m} \sqrt{k k^{\prime}} \frac{f\left(k, k^{\prime}\right)}{\sqrt{f(k, k) f\left(k^{\prime}, k^{\prime}\right)}} .
$$

With this procedure the term $\sqrt{k k^{\prime}}$ for Kijowski's distribution naturally arises in combination with the model-dependent term

$$
F\left(k, k^{\prime}\right)=\frac{f\left(k, k^{\prime}\right)}{\sqrt{f(k, k) f\left(k^{\prime}, k^{\prime}\right)}} .
$$

For a connection to ideal quantities, $F\left(k, k^{\prime}\right)$ has to be investigated in specific parameter limits. 


\subsection{Operator normalization for narrow laser fields}

The first-photon distribution $\Pi(t)$ of a two-level atom impinging from the left on a half-space laser field has been shown to be related to Kijowski's distribution by means of a normalization on the level of operators in the limit $\gamma \rightarrow \infty, \gamma / \Omega=$ const. As indicated in Section 3.3, a very interesting question is the generalization for particles with arbitrary momentum support coming in as a coherent superposition from both sides. Before this is to be investigated one has to check whether the operator normalization also yields Kijowski's distribution for narrow laser fields.

For this, one starts with the operator corresponding to $\Pi_{\delta}(t)$ as in Section 5.1,

$$
\Pi_{\delta}(t)=\left\langle 1\left|\left\langle\psi\left|\widehat{\Pi}_{\delta, t}\right| \psi\right\rangle\right| 1\right\rangle
$$

where

$$
\widehat{\Pi}_{\delta, t}=\gamma U_{\mathrm{c}}^{\mathrm{I}}(t,-\infty)^{\dagger}|2\rangle\langle 2| U_{\mathrm{c}}^{\mathrm{I}}(t,-\infty)
$$

and $U_{\mathrm{c}}^{\mathrm{I}}(t,-\infty)$ is given by Eq. (5.1) with the conditional Hamiltonian of Eq. (3.116). With Eq. (3.124) it follows that

$$
U_{\mathrm{c}}^{\mathrm{I}}(\infty,-\infty)|\psi\rangle|1\rangle=\int_{0}^{\infty} \mathrm{d} k \widetilde{\psi}(k)\left(R_{1}(k)|-k\rangle+T_{1}(k)|k\rangle\right)|1\rangle .
$$

Then Eqs. (5.4) and (5.8) provide the normalization operator $\widehat{B}=\int_{-\infty}^{\infty} \mathrm{d} t \widehat{\Pi}_{\delta, t}$, acting on the incoming states with internal ground state. It takes in the $k$-space the form

$$
\left\langle 1\left|\left\langle k|\widehat{B}| k^{\prime}\right\rangle\right| 1\right\rangle=\left(1-\overline{R_{1}(k)} R_{1}\left(k^{\prime}\right)-\overline{T_{1}(k)} T_{1}\left(k^{\prime}\right)\right) \delta\left(k-k^{\prime}\right)
$$

Defining the operator normalized first-photon distribution for a Dirac delta-like laser shape by

$$
\Pi_{\delta}^{\mathrm{ON}}(t)=\left\langle 1\left|\left\langle\psi\left|\widehat{B}^{-1 / 2} \widehat{\Pi}_{\delta, t} \widehat{B}^{-1 / 2}\right| \psi\right\rangle\right| 1\right\rangle,
$$

an evaluation in $k$-space leads to

$$
\begin{array}{rl}
\Pi_{\delta}^{\mathrm{ON}}(t)=\gamma \int_{-\infty}^{\infty} \mathrm{d} & x \int_{0}^{\infty} \mathrm{d} k \mathrm{~d} k^{\prime} \overline{\widetilde{\psi}(k)} \widetilde{\psi}\left(k^{\prime}\right) \mathrm{e}^{\mathrm{i} \hbar\left(k^{2}-k^{\prime 2}\right) t / 2 m} \overline{\phi_{k}^{(2)}(x)} \phi_{k^{\prime}}^{(2)}(x) \\
& \times\left(1-\left|R_{1}(k)\right|^{2}-\left|T_{1}(k)\right|^{2}\right)^{-1 / 2}\left(1-\left|R_{1}\left(k^{\prime}\right)\right|^{2}-\left|T_{1}\left(k^{\prime}\right)\right|^{2}\right)^{-1 / 2} .
\end{array}
$$

Here the stationary states $\phi_{k}^{(2)}(x)$ are given by Eq. (3.118) and the transmission and reflection coefficients of the ground state for a delta laser potential are given by Eqs. (3.120) and (3.122).

To relate $\Pi_{\delta}^{\mathrm{ON}}(t)$ to an ideal arrival-time distribution, the limit $\gamma \rightarrow \infty$ with $\gamma / \Omega=$ const. has to be applied. With

$$
\beta=\gamma / \Omega=\text { const. }
$$


one has in leading order

$$
\begin{aligned}
R_{1}(k) & \sim-1+\frac{4 \hbar^{2} \beta^{2}}{m^{2}} \sqrt{\frac{\mathrm{i} m}{\hbar}} \gamma^{-3 / 2} k, \\
T_{1}(k) & \sim \frac{4 \hbar^{2} \beta^{2}}{m^{2}} \sqrt{\frac{\mathrm{i} m}{\hbar}} \gamma^{-3 / 2} k \\
\left(1-\left|R_{1}\right|^{2}-\left|T_{1}\right|^{2}\right)^{-1 / 2} & \sim\left(\frac{m \gamma}{2 \hbar}\right)^{3 / 4} \frac{1}{\beta \sqrt{2 k}} \\
\gamma \int_{-\infty}^{\infty} \mathrm{d} x \overline{\phi_{k}^{(2)}(x)} \phi_{k^{\prime}}^{(2)}(x) & \sim \frac{1}{2 \pi}\left(\frac{2 \hbar}{m}\right)^{5 / 2} \frac{\beta^{2}}{\gamma^{3 / 2}} k k^{\prime} .
\end{aligned}
$$

Inserting these expansions into Eq. (5.43) yields exactly Kijowski's distribution. Thus, the same remarkable result holds for narrow laser fields as well as for the half-space laser, namely

$$
\Pi_{\delta}^{\mathrm{ON}}(t) \rightarrow \Pi_{\mathrm{K}}(t), \quad \gamma \rightarrow \infty, \gamma / \Omega=\text { const. }
$$

In principle, the approach could be applied to wave packets coming in from the left and from the right. However, it is more convenient to do this in the one-channel limit. For that purpose, operator normalization for absorbing potentials is introduced in the following section. The question of arrivals for arbitrary momentum support is then treated in Chapter 6.

\subsection{Operator normalization for absorbing poten- tials}

It is clear from the connection between absorbing potentials and the two-channel laser model that operator normalization is applicable as well to the one-channel case. For the sake of completeness the result of operator-normalizing the arrivaltime distribution of Eq. (3.144) is given here for the case of a half-space absorbing potential. Narrow potentials in this connection are investigated in Chapter 6.

Consider the case of a purely absorbing half-space potential, $L \rightarrow \infty$, as described in Section 3.4.1. The Hamiltonian for this problem is given by

$$
H=\frac{\widehat{p}^{2}}{2 m}-\mathrm{i} V_{0} \Theta(\widehat{x})
$$

and its eigenfunctions $\phi_{k}$ for a plane wave incoming from the left are

$$
\phi_{k}(x)=\frac{1}{\sqrt{2} \pi} \begin{cases}\mathrm{e}^{\mathrm{i} k x}+R(k) \mathrm{e}^{-\mathrm{i} k x}, & x \leq 0 \\ T(k) \mathrm{e}^{\mathrm{i} k x}, & x \geq 0\end{cases}
$$

The reflection and transmission coefficients are obtained in Section B.1 of the appendix and for the imaginary potential $V_{0}=\hbar \Omega^{2} / 2 \gamma$ they are given with Eqs. (B.17) 
and (B.18) by

$$
\begin{aligned}
R(k) & =\frac{k-\kappa}{k+\kappa}, \\
T(k) & =\frac{2 k}{k+\kappa}, \\
\kappa & =\sqrt{k^{2}+2 \mathrm{i} m V_{0} / \hbar^{2}}, \quad \operatorname{Im} \kappa>0 .
\end{aligned}
$$

Eq. (3.144) yields the unnormalized arrival-time distribution in terms of the absorption rate for the wave packet $\left|\psi_{t}\right\rangle=\int_{0}^{\infty} \mathrm{d} k \widetilde{\psi}(k) \mathrm{e}^{-\mathrm{i} \hbar k^{2} t / 2 m}\left|\phi_{k}\right\rangle$,

$$
\Pi(t)=\frac{2 V_{0}}{\hbar} \int_{0}^{\infty} \mathrm{d} x|\psi(x, t)|^{2} .
$$

This expression can be written in terms of an operator $\widehat{\Pi}_{t}$,

$$
\Pi(t)=\left\langle\psi\left|\widehat{\Pi}_{t}\right| \psi\right\rangle=\frac{2 V_{0}}{\hbar}\left\langle\psi\left|U_{\mathrm{c}}^{\mathrm{I}}(t,-\infty)^{\dagger} \Theta(\widehat{x}) U_{\mathrm{c}}^{\mathrm{I}}(t,-\infty)\right| \psi\right\rangle
$$

Following the approach of Section 5.1, the normalized distribution is

$$
\Pi^{\mathrm{ON}}(t)=\left\langle\psi\left|\widehat{\Pi}_{t}^{\mathrm{ON}}\right| \psi\right\rangle=\left\langle\psi\left|\widehat{B}^{-1 / 2} \widehat{\Pi}_{t} \widehat{B}^{-1 / 2}\right| \psi\right\rangle .
$$

For the normalization operator $\widehat{B}$, one notes that for large times only the reflected packet remains,

$$
U_{\mathrm{c}}^{\mathrm{I}}(\infty,-\infty)|\psi\rangle=\int_{0}^{\infty} \mathrm{d} k \widetilde{\psi}(k) R(k)|-k\rangle,
$$

and thus with Eqs. (5.3),(5.9) and (5.57)

$$
\begin{aligned}
\Pi^{\mathrm{ON}}(t)=\frac{2 V_{0}}{\hbar} \int_{0}^{\infty} \mathrm{d} x \int_{0}^{\infty} \mathrm{d} k \mathrm{~d} k^{\prime} \overline{\widetilde{\psi}(k)} \widetilde{\psi}\left(k^{\prime}\right)\left(1-|R(k)|^{2}\right)^{-1 / 2}\left(1-\left|R\left(k^{\prime}\right)\right|^{2}\right)^{-1 / 2} \\
\times \overline{T(k)} T\left(k^{\prime}\right) \mathrm{e}^{\mathrm{i} \hbar\left(k^{2}-k^{\prime 2}\right) t / 2 m} \mathrm{e}^{-\mathrm{i}\left(\bar{\kappa}-\kappa^{\prime}\right) x} .
\end{aligned}
$$

Now the limit for vanishing delay in the absorption rate has to be applied, namely $V_{0} \rightarrow \infty$. This yields

$$
\begin{aligned}
\kappa & \sim \sqrt{2 \mathrm{i} m V_{0} / \hbar^{2}}, \\
R(k) & \sim-1+\frac{\hbar k}{\sqrt{m V_{0}}}(1-\mathrm{i}), \\
1-|R(k)|^{2} & \sim \frac{2 \hbar k}{\sqrt{m V_{0}}}, \\
T(k) & \sim 2 k / \kappa, \quad V_{0} \rightarrow \infty .
\end{aligned}
$$

Inserting these expressions into Eq. (5.59) and integrating over $x$ leads again to Kijowski's distribution,

$$
\Pi^{\mathrm{ON}}(t) \rightarrow \Pi_{\mathrm{K}}(t), \quad V_{0} \rightarrow \infty
$$


Chapter 5. Operator normalization

Thus, the operator normalization establishes the connection between the absorption rate in an imaginary potential and Kijowski's arrival-time distribution in exactly the same manner as for the first-photon distribution.

The advantage of the one-channel model is that it provides a simple calculational tool for further, more complicated, arrival-time problems, e.g. in the presence of realvalued external potentials. This will be investigated in Chapter 6 .

\subsection{Measurement-based approaches to operator normalization}

In Chapter 5.1 it has been shown that the arrival time distribution of Kijowski, $\Pi_{K}(t)$, can be related to the operational quantity of a temporal first-photon distribution by means of a normalization of the corresponding operators. Reasonable as this approach may be, an important pending question is its understanding in terms of some measuring procedure.

In this section it is shown that the mathematical tool of an operator normalization can be understood physically by an ideal filtering operation on the incoming wave packet. The characteristic features of such a filtering are modeled and explicit expressions for real-valued filter potentials are found by means of inverse scattering theory.

\subsubsection{Operator normalization and filtering}

For convenience, arrival-time distributions are operationally described in this section by means of the absorption rate in a purely imaginary half-space potential $V(x)=-\mathrm{i} V_{0} \Theta(x)$. A generalization to other models, e.g. narrow potentials, is straightforward. The unnormalized absorption rate for a wave packet $\left|\psi_{t}\right\rangle$ coming in from the left to the measurement potential has been derived in Section 3.4.1 and it is given with Eq. (3.144) and in the limit $L \rightarrow \infty$ by

$$
\Pi(t)=\frac{2 V_{0}}{\hbar} \int_{0}^{\infty} \mathrm{d} x|\psi(x, t)|^{2} .
$$

The operator-normalized version of this operational quantity takes in the limit of strong detection $V_{0} \rightarrow \infty$ and with Eqs. (5.59) and (5.60) the form

$$
\Pi^{\mathrm{ON}}(t) \sim\left|\int_{0}^{\infty} \mathrm{d} k \widetilde{\psi}(k) f(k) k \mathrm{e}^{-\mathrm{i} \hbar k^{2} t / 2 m}\right|^{2}
$$

with

$$
f(k) \sim k^{-1 / 2}, \quad V_{0} \rightarrow \infty
$$

Consequently, one has

$$
\Pi^{\mathrm{ON}}(t) \rightarrow \Pi_{\mathrm{K}}(t), \quad V_{0} \rightarrow \infty
$$


since constant factors cancel for the sake of normalization. The change from $\widetilde{\psi}(k)$ to $\widetilde{\psi}(k) f(k)$ can be viewed as a filtering operation acting on the incoming momentum distribution. In the context of one-dimensional scattering, the filtering has to be understood as an additional interaction of the incoming wave packet with a filter potential, such that in the asymptotic reflection or transmission regime of the filter potential the momentum distribution has changed from $\widetilde{\psi}(k)$ to $\widetilde{\psi}(k) f(k)$. A perfect filter leading to the distribution $\Pi_{K}(t)$ is given by

$$
f_{\text {id }}(k) \sim k^{-1 / 2},
$$

but, due to its unbound nature for $k \rightarrow 0$, this ideal filter operation $f_{\text {id }}(k)$ has no physical meaning.

However, for a momentum distribution $\widetilde{\psi}(k)$, sharply peaked around $k=k_{0}$, it is assumed to be sufficient to approximate the ideal filter by a physical one at $k=k_{0}$. Since $f_{\text {id }}(k) \rightarrow 0$ for $k \rightarrow \infty$, the construction of a reflecting filter potential is advantageous, since the reflection coefficient of a usual scattering problem tends to zero for large $k$, too, in contrast to the transmission coefficient. The basic setup is schematically shown in Fig. 5.4. The incoming direction of the packet into the

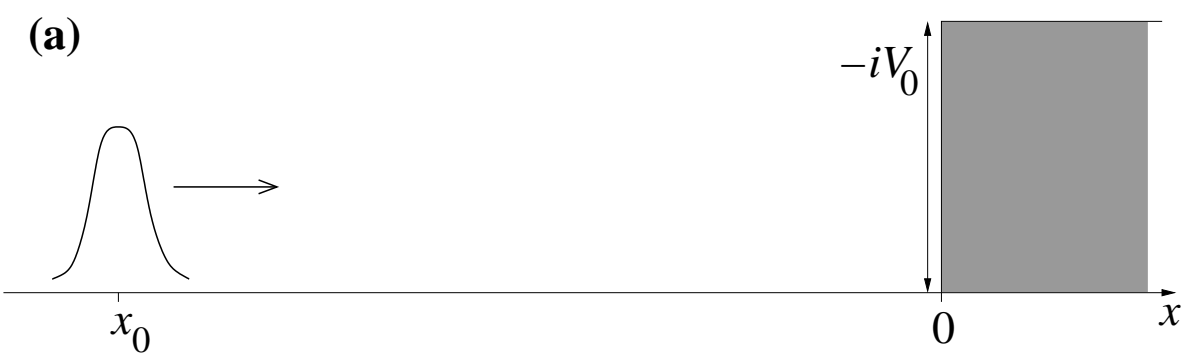

(b)

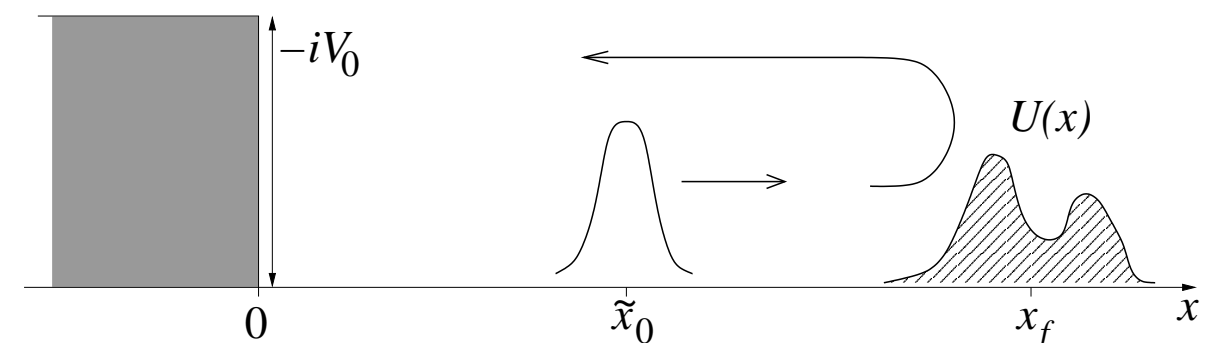

Figure 5.4: Arrival-time measurement with an absorbing potential $V(x)=$ $-\mathrm{i} V_{0} \Theta( \pm x)$ for (a) a free incoming wave packet and (b) a pre-filtered wave packet with filter potential $U(x)$ located at $x_{\mathrm{f}}$.

measurement potential does not play any role, because the detection only depends on the transmission coefficient which is the same both for right and for left incidence. To obtain the same phase in the reflected wave packet as for the free case, the reference point $x_{\mathrm{f}}$ of the filter potential and the mean position $\tilde{x}_{0}$ of the initial packet are connected through

$$
\left|x_{0}\right|=2 x_{\mathrm{f}}-\tilde{x}_{0} .
$$


Chapter 5. Operator normalization

Furthermore, it is assumed that the separation of the filter potential and the imaginary measurement potential are large compared to the width $\Delta x$ of the wave packet,

$$
\Delta x \ll \tilde{x}_{0} \ll x_{\mathrm{f}} .
$$

This guarantees that the filtering can be seen as part of the preparation procedure with respect to the measurement. In the following sections, explicit filter potentials $U(x)$ are constructed, which exhibit reflection coefficients close to $f_{\text {id }}(k)$ in a region around $k=k_{0}$.

\subsubsection{Real-valued filter potentials}

The construction of real filter potentials is favored, since in that case a physical realization is evident. For example, they may be realized by far detuned laser fields with an appropriate shape according to Eq. (3.140). For real potentials, the reflection coefficient $R(k)$ has to fulfill [116]

$$
\begin{aligned}
& R(k)=\overline{R(-k)} \\
& R(k) \leq 1
\end{aligned}
$$

The first condition is represented by an ideal filter function $f_{\text {id }}(k)$ of the form

$$
f_{\text {id }}(k)=(\mathrm{i} k)^{-1 / 2}=\mathrm{e}^{-\mathrm{i} \pi / 4} k^{-1 / 2} .
$$

The constant phase factor assures $f_{\text {id }}(k)=\overline{f_{\text {id }}(-k)}$ and it drops out in the expression (5.63) for the arrival time distribution.

As an approximation of $f_{\text {id }}(k)$ the following expression is proposed:

$$
f_{N}(k)=\sum_{n=0}^{N-1} a_{n}(\mathrm{i} k)^{n}\left[\sum_{n=0}^{N-1} b_{n}(\mathrm{i} k)^{n}+(\mathrm{i} k)^{N}\right]^{-1}
$$

with $a_{n}, b_{n} \in \mathbb{R}$. This ansatz reproduces the properties $f_{N}(k)=\overline{f_{N}(-k)}$ and $f_{N}(k) \rightarrow 0$ for $k \rightarrow \infty$. Moreover, its form as a rational function in $k$ is well suited for inverse scattering methods which will become important in the following. Denoting by $f_{N}^{\text {re }}, f_{N}^{\text {im }}$ the real and imaginary part of $f_{N}$ and by $f_{\text {id }}^{\text {re }}, f_{\text {id }}^{\text {im }}$ the real and imaginary part of $f_{\text {id }}$, the unknown coefficients $a_{n}$ and $b_{n}$ can be determined in a way such that the first $2(N-1)$ derivations of $f_{\text {id }}$ and $f_{N}$ coincide at $k=k_{0}$, i.e.

$$
\left(\frac{\mathrm{d}^{m} f_{\mathrm{id}}^{\mathrm{re}, \mathrm{im}}(k)}{\mathrm{d} k^{m}}\right)_{k=k_{0}}=\left(\frac{\mathrm{d}^{m} f_{N}^{\mathrm{re}, \mathrm{im}}(k)}{\mathrm{d} k^{m}}\right)_{k=k_{0}}, \quad m=0, \ldots, 2(N-1) .
$$

Eqs. (5.73) can be solved analytically for $N=1$, leading to

$$
f_{1}(k)=\frac{-\mathrm{i} \sqrt{2 k_{0}}}{k-\mathrm{i} k_{0}}
$$


but for larger $N$ a numerical treatment for a particular value of $k_{0}$ is required. To give an example, in the following a value of $k_{0}=0.42$ is chosen (all numbers in atomic units, see Appendix D), which corresponds to the example considered previously in Fig. 3.5. Solving Eqs. (5.73) for this value of $k_{0}$ yields

$$
a_{0} \simeq-0.77, \quad a_{1} \simeq-1.83, \quad b_{0} \simeq-0.18, \quad b_{1} \simeq-1.68 .
$$

The modulus of the corresponding filter functions $f_{N}(k)$ are compared with the ideal filter in Fig. 5.5.

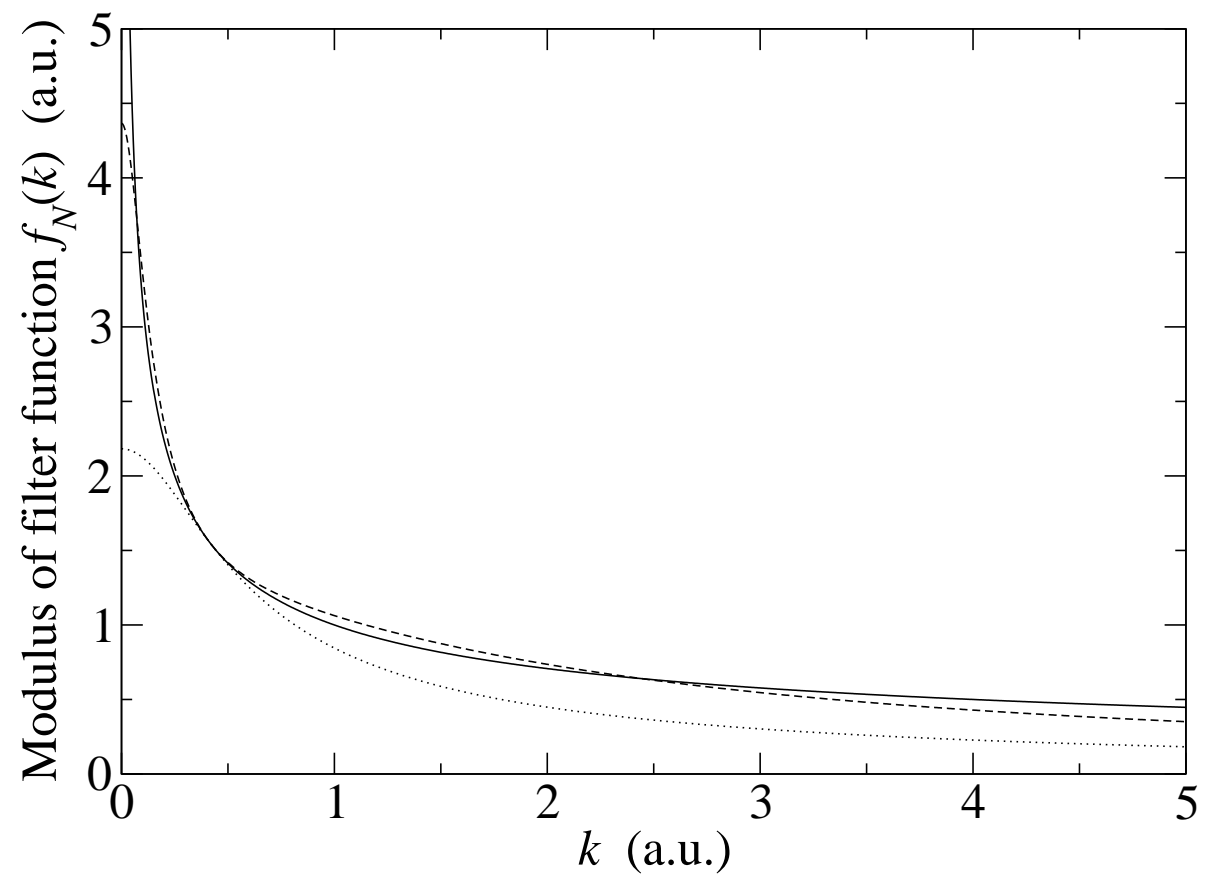

Figure 5.5: Approximations $\left|f_{N}(k)\right|$ to the ideal filter function $\left|f_{\text {id }}(k)\right|=k^{-1 / 2}$ (solid line) for $N=1$ (dotted line) and $N=2$ (dashed line), $k_{0}=0.42$.

To incorporate the condition (5.70), the functions $f_{N}(k)$ are normalized with respect to the condition $R_{N}(0)=-1$ [116],

$$
R_{N}(k)=-\frac{f_{N}(k)}{f_{N}(0)}
$$

The normalization constant does not play any role for the arrival-time measurement, since it drops out in Eq. (5.63) after an ordinary normalization. With Eqs. (5.74) and (5.75) one has

$$
\begin{aligned}
& R_{1}(k)=\frac{\mathrm{i} k_{0}}{k-\mathrm{i} k_{0}}, \\
& R_{2}(k)=-\frac{b_{0}}{a_{0}} \frac{a_{0}+\mathrm{i} a_{1} k}{b_{0}+\mathrm{i} a_{0} k+k^{2}} .
\end{aligned}
$$

These expressions determine the required filter potentials $U_{N}(x)$ : the $U_{N}(x)$ have to "produce" the given reflection coefficients. Before this problem will be solved 
Chapter 5. Operator normalization

by means of inverse scattering methods, the consequences of the filtering for an arrival-time measurement are investigated.

In the following, the filter potential $U_{N}(x)$ and the corresponding desired reflection coefficient $R_{N}(k)$ shall be given and the setup is assumed to be as illustrated in Fig. 5.4. The initial wave packet is prepared at time $t=0$ at $\langle x\rangle=\tilde{x}_{0}$ with only positive momentum components; it impinges on the filter potential and it becomes partially reflected. Far away from the filter potential the reflected part is described by

$$
\psi_{R}(x, t)=\int_{0}^{\infty} \mathrm{d} k \widetilde{\psi}(k) R_{N}(k) \mathrm{e}^{-\mathrm{i} k x}
$$

This is the wave packet whose arrival-time distribution is measured by means of its absorption rate in the imaginary potential. In the limit $V_{0} \rightarrow \infty$ and properly normalized to 1 with a normalization constant $P_{N}$, the measured arrival-time distribution reads

$$
\Pi_{N}(t)=P_{N}^{-1}\left|\int_{0}^{\infty} \mathrm{d} k \widetilde{\psi}(k) R_{N}(k) k \mathrm{e}^{-\mathrm{i} \hbar k^{2} t / 2 m}\right|^{2}
$$

Since one has with Eq. (5.73) $f_{N}(k) \rightarrow f_{\text {id }}(k) \sim k^{-1 / 2}$ for $N \rightarrow \infty$, and $R_{N}(k) \sim$ $f_{N}(k)$, the following limit holds:

$$
\lim _{N \rightarrow \infty} \Pi_{N}(t)=\Pi_{\mathrm{K}}(t)
$$

This is shown in Fig. 5.6 for the same parameter values as in Fig. 3.5 and for finite $N=1,2$. For $N=2$, the agreement with Kijowski's distribution is very good indicating that the second order approximation for the filter potential may be sufficient for some examples.

It remains to derive the corresponding filter potentials. Since the reflection coefficients are constructed as rational functions in $k$, this is always possible and standard methods exist [117]. The following approach relies on the Faddeev-Marchenko inverse scattering theory. It is briefly outlined in Appendix C.

The case $N=1$ : The reflection coefficient from the left, $R_{\mathrm{L}}(k)$, is given by Eq. (5.77). It has a single pole in the upper-half complex plane at $k=\mathrm{i} k_{0}$. Although the potential is easy to recognize as the attractive delta function potential, a full derivation in terms of inverse scattering theory is given to introduce the methods. To derive the corresponding transmission coefficient with the methods of Appendix C.2, it is assumed that $T_{1}(k)$ also has a pole at $k=\mathrm{i} k_{0}$, and one first considers the modified function

$$
\tilde{T}_{1}(k)=T_{1}(k) \frac{k-\mathrm{i} k_{0}}{k+\mathrm{i} k_{0}} .
$$




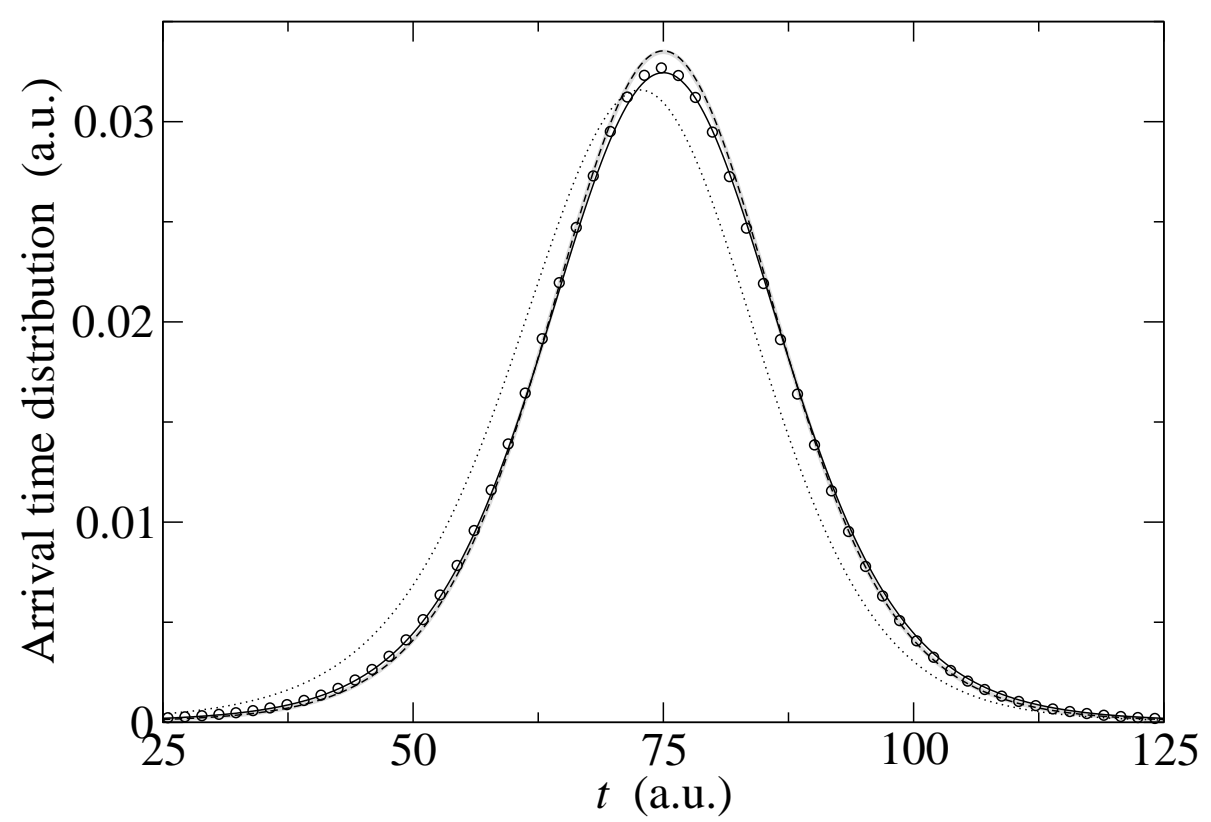

Figure 5.6: Comparison of the axiomatic arrival-time distribution of Kijowski, $\Pi_{\mathrm{K}}(t)$, (solid line) with the normalized absorption rate without filter, $\Pi(t)$ (dashed line), and with pre-filtered wave packet for $N=1$ (dotted line) and $N=2$ (circles). The Gaussian wave packet is chosen to have minimum-uncertainty when its center arrives at $x=0, t=75$, to enhance the difference between $\Pi_{\mathrm{K}}(t)$ and the flux (grey line); $k_{0}=0.42, \Delta x=5$ in atomic units.

It is given with Eq. (C.10) by

$$
\begin{aligned}
\frac{\mathrm{d}}{\mathrm{d} k} \ln \tilde{T}_{1}(k) & =\frac{1}{2 \pi \mathrm{i}} \int_{-\infty}^{\infty} \mathrm{d} \xi \frac{2 k_{0}}{\left(\xi-k+\mathrm{i} 0^{+}\right)\left(\xi^{2}+k_{0}^{2}\right) \xi} \\
& =\frac{1}{2 \pi \mathrm{i}} \mathcal{P} \int_{-\infty}^{\infty} \mathrm{d} \xi \frac{2 k_{0}}{(\xi-k)\left(\xi^{2}+k_{0}^{2}\right) \xi}+\frac{1}{2} \int_{-\infty}^{\infty} \mathrm{d} \xi \frac{2 k_{0}}{\xi\left(\xi^{2}+k_{0}^{2}\right)} \delta(\xi-k) \\
& =\frac{1}{k}-\frac{1}{k+\mathrm{i} k_{0}}
\end{aligned}
$$

where $\mathcal{P}$ denotes a principal value integration. It follows that $\ln \tilde{T}_{1}(k)=\ln k-\ln (k+$ $\left.\mathrm{i} k_{0}\right)+C$, where the integration constant $C$ is zero due to the condition $\tilde{T}_{1}(k) \rightarrow 1$ for $k \rightarrow \infty$. This finally yields $\tilde{T}_{1}(k)=k /\left(k+\mathrm{i} k_{0}\right)$ or, with Eq. (5.82),

$$
T_{1}(k)=\frac{k}{k-\mathrm{i} k_{0}}
$$

The Fourier transform of $R_{\mathrm{L}}(k)$, defined by Eq. (C.12), is given by

$$
r_{\mathrm{L}}(z)= \begin{cases}-k_{0} \mathrm{e}^{k_{0} z}, & z<0 \\ 0, & z>0\end{cases}
$$


and with Eq. (C.18) one has

$$
s_{\mathrm{L}}(z)= \begin{cases}0, & z<0 \\ k_{0} \mathrm{e}^{k_{0} z}, & z>0 .\end{cases}
$$

Inserting $s_{\mathrm{L}}(z)$ into the left Marchenko equation (C.20) yields for $x+y>0$

$$
k_{0} \mathrm{e}^{k_{0}(x+y)}+A_{\mathrm{L}}(x, y)+\int_{-y}^{x} \mathrm{~d} x^{\prime} A_{\mathrm{L}}\left(x, x^{\prime}\right) k_{0} \mathrm{e}^{k_{0}\left(x^{\prime}+y\right)}=0,
$$

and this equation is easily solved by setting $A_{\mathrm{L}}(x, y)=-k_{0}$. For $x+y<0, s_{\mathrm{L}}(x+y)$ vanishes and the solution of Eq. (5.87) is $A_{\mathrm{L}}(x, y)=0$. When combining these two results, one has

$$
A_{\mathrm{L}}(x, y)=-k_{0} \Theta(x+y),
$$

and with Eq. (C.17) the potential reads

$$
U_{1}(x)=\frac{\mathrm{d}}{\mathrm{d} x} A_{\mathrm{L}}(x, x)=-k_{0} \frac{\mathrm{d}}{\mathrm{d} x} \Theta(2 x)=-k_{0} \delta(x) .
$$

This is the expected result for the reflection coefficient of Eq. (5.77). Thus, the first order filter potential is an attractive delta potential which can be approximately realized for example by a narrow Gaussian potential.

The case $N=2$ : The reflection coefficient from the left is given with Eq. (5.78) by

$$
R_{2}(k) \equiv R_{\mathrm{L}}(k)=-\frac{b_{0}}{a_{0}} \frac{a_{0}+\mathrm{i} a_{1} k}{b_{0}+\mathrm{i} b_{1} k+k^{2}} .
$$

This expression can be rewritten by identifying the poles of the numerator and the denominator. By defining

$$
\begin{aligned}
& \alpha=-\frac{b_{1}}{2}+\sqrt{\frac{b_{1}^{2}}{4}+b_{0}} \\
& \beta=-\frac{b_{1}}{2}-\sqrt{\frac{b_{1}^{2}}{4}+b_{0}} \\
& \zeta=\frac{a_{0}}{a_{1}},
\end{aligned}
$$

it follows that

$$
R_{2}(k) \equiv R_{\mathrm{L}}(k)=\frac{\mathrm{i} \alpha \beta}{\zeta} \frac{k-\mathrm{i} \zeta}{(k-\mathrm{i} \alpha)(k-\mathrm{i} \beta)} .
$$

Note that for the particular choice of the parameters given in Eq. (5.75) one has $\alpha>0, \beta>0$ and $\zeta>0$, i.e. $R_{\mathrm{L}}(k)$ has two poles in the upper-half complex plane. To derive the corresponding transmission coefficient, $T_{2}(k)$, the quantity

$$
\tilde{T}_{2}(k)=T_{2}(k) \frac{k-\mathrm{i} \alpha}{k+\mathrm{i} \alpha} \frac{k-\mathrm{i} \beta}{k+\mathrm{i} \beta}
$$


is considered which is determined by Eq. (C.10). After some calculation and with

$$
\sigma^{2}=\alpha^{2}+\beta^{2}-\alpha^{2} \beta^{2} / \zeta^{2}
$$

it follows with Eqs. (C.10) and (5.90) that

$$
\begin{aligned}
\frac{\mathrm{d}}{\mathrm{d} k} \ln \tilde{T}_{2}(k)= & \frac{1}{2 \pi \mathrm{i}} \frac{2 \alpha^{2} \beta^{2}}{\zeta^{2}} \int_{-\infty}^{\infty} \mathrm{d} \xi \frac{\xi^{4}+2 \xi^{2} \zeta^{2}+\zeta^{2} \sigma^{2}}{\left(\xi-k+\mathrm{i} 0^{+}\right)\left(\xi^{2}+\alpha^{2}\right)\left(\xi^{2}+\beta^{2}\right)\left(\xi^{2}+\sigma^{2}\right) \xi} \\
= & \frac{1}{2 \pi \mathrm{i}} \frac{2 \alpha^{2} \beta^{2}}{\zeta^{2}} \mathcal{P} \int_{-\infty}^{\infty} \mathrm{d} \xi \frac{\xi^{4}+2 \xi^{2} \zeta^{2}+\zeta^{2} \sigma^{2}}{(\xi-k)\left(\xi^{2}+\alpha^{2}\right)\left(\xi^{2}+\beta^{2}\right)\left(\xi^{2}+\sigma^{2}\right) \xi} \\
& \quad+\frac{1}{2} \frac{2 \alpha^{2} \beta^{2}}{\zeta^{2}} \int_{-\infty}^{\infty} \mathrm{d} \xi \frac{\xi^{4}+2 \xi^{2} \zeta^{2}+\zeta^{2} \sigma^{2}}{\left(\xi^{2}+\alpha^{2}\right)\left(\xi^{2}+\beta^{2}\right)\left(\xi^{2}+\sigma^{2}\right) \xi} \delta(\xi-k) \\
= & \frac{\mathrm{i} \alpha}{k^{2}+\alpha^{2}}+\frac{\mathrm{i} \beta}{k^{2}+\beta^{2}}-\frac{\mathrm{i} \sigma}{k^{2}+\sigma^{2}}-\frac{k}{k^{2}+\alpha^{2}}-\frac{k}{k^{2}+\beta^{2}}+\frac{k}{k^{2}+\sigma^{2}}+\frac{1}{k},
\end{aligned}
$$

where in the last step, involving the principal value integration and the expansion into partial fractions, the computer-algebra program MAPLE has been used. Integrating Eq. (5.97) over $k$ yields

$$
\begin{aligned}
\ln \tilde{T}_{2}(k)=\mathrm{i} \arctan \left(\frac{k}{\alpha}\right)+\mathrm{i} \arctan \left(\frac{k}{\beta}\right)-\mathrm{i} \arctan \left(\frac{k}{\sigma}\right) \\
-\frac{1}{2} \ln \left(k^{2}+\alpha^{2}\right)-\frac{1}{2} \ln \left(k^{2}+\beta^{2}\right)+\frac{1}{2} \ln \left(k^{2}+\sigma^{2}\right)+\ln (k)+C .
\end{aligned}
$$

The integration constant $C$ is determined by the limit $k \rightarrow \infty$, for which one obtains $0=\mathrm{i} \pi / 2+C$ and thus $C=-\mathrm{i} \pi / 2=\ln (-\mathrm{i})$. Solving Eq. (5.98) for $\tilde{T}_{2}(k)$ one obtains

$$
\tilde{T}_{2}(k)=\frac{k(k+\mathrm{i} \sigma)}{(k+\mathrm{i} \alpha)(k+\mathrm{i} \beta)},
$$

and with Eq. (5.95) the transmission coefficient finally reads

$$
T_{2}(k)=\frac{k(k+\mathrm{i} \sigma)}{(k-\mathrm{i} \alpha)(k-\mathrm{i} \beta)} .
$$

$T_{2}(k)$ has two poles in the positive imaginary plane at $k=\mathrm{i} \alpha$ and $k=\mathrm{i} \beta$, as expected, such that the potential $U_{2}(x)$ will have two bound states. It can be easily shown that $\left|R_{2}(k)\right|^{2}+\left|T_{2}(k)\right|^{2}=1$. To reconstruct $U_{2}(x)$ for $x>0$, it is advantageous to proceed with the reflection coefficient from the right, given with Eq. (C.9) by

$$
R_{\mathrm{R}}(k)=\frac{-R_{\mathrm{L}}(-k) T(k)}{T(-k)}=\frac{\mathrm{i} \alpha \beta}{\zeta} \frac{(k+\mathrm{i} \zeta)(k+\mathrm{i} \sigma)}{(k-\mathrm{i} \alpha)(k-\mathrm{i} \beta)(k-\mathrm{i} \sigma)} .
$$

Its Fourier transform is given with Eq. (C.13) by

$$
\begin{array}{r}
r_{\mathrm{R}}(z)=\frac{\alpha \beta}{\zeta}\left(\frac{(\alpha+\zeta)(\alpha+\sigma)}{(\alpha-\beta)(\sigma-\alpha)} \mathrm{e}^{-\alpha z}-\frac{(\beta+\zeta)(\beta+\sigma)}{(\alpha-\beta)(\sigma-\beta)} \mathrm{e}^{-\beta z}+\frac{2 \sigma(\sigma+\zeta)}{(\alpha-\sigma)(\sigma-\beta)} \mathrm{e}^{-\sigma z}\right) \\
\times \Theta(z) .
\end{array}
$$


Chapter 5. Operator normalization

For the right Marchenko equation, the quantity $s_{\mathrm{R}}(z)$ is required, and it is given by Eq. (C.19). One has for $z>0$

$$
\begin{aligned}
s_{\mathrm{R}}(z) & =r_{\mathrm{R}}(z)-\mathrm{i} \underset{\mathrm{i} \alpha}{\operatorname{Res}}[T(k)] \frac{R_{\mathrm{R}}(\mathrm{i} \alpha)}{T(\mathrm{i} \alpha)} \mathrm{e}^{-\alpha z}-\mathrm{i} \underset{\mathrm{i} \beta}{\operatorname{Res}}[T(k)] \frac{R_{\mathrm{R}}(\mathrm{i} \beta)}{T(\mathrm{i} \beta)} \mathrm{e}^{-\beta z} \\
& =\frac{2 \alpha \beta \sigma(\sigma-\zeta)}{\zeta(\alpha-\sigma)(\sigma-\beta)} \mathrm{e}^{-\sigma z}=: M \mathrm{e}^{-\sigma z}, \quad z>0,
\end{aligned}
$$

where $M$ has been defined for convenience. The right Marchenko equation (C.21) for $x+y>0$ reads

$$
M \mathrm{e}^{-\sigma(x+y)}+A_{\mathrm{R}}(x, y)+\int_{x}^{\infty} \mathrm{d} x^{\prime} M \mathrm{e}^{-\sigma\left(x^{\prime}+y\right)} A_{\mathrm{R}}\left(x, x^{\prime}\right)=0, \quad x<y .
$$

Eq. (5.104) can be solved by the separation ansatz $A_{\mathrm{R}}(x, y)=a(x) \mathrm{e}^{-\sigma y}$ [118]. Inserting this ansatz into the integral equation (5.104), integrating and solving for $a(x)$ yields

$$
a(x)=-\frac{M \mathrm{e}^{-\sigma x}}{1+\frac{M}{2 \sigma} \mathrm{e}^{-2 \sigma x}},
$$

and thus

$$
A_{\mathrm{R}}(x, x)=\frac{-M}{\mathrm{e}^{2 \sigma x}+\frac{M}{2 \sigma}}, \quad x>0 .
$$

The filter potential for positive $x$ is finally obtained with Eq. (C.16) and with

$$
\mathrm{e}^{-\phi}=\frac{M}{2 \sigma}
$$

as

$$
U_{2}(x)=-\frac{\mathrm{d}}{\mathrm{d} x} A_{\mathrm{R}}(x, x)=-\sigma^{2} \cosh ^{-2}(\sigma x+\phi / 2), \quad x>0 .
$$

For negative $x$, the left Marchenko equation (C.20) is used. The Fourier transform of the left reflection coefficient $R_{\mathrm{L}}(k)$ is given with Eq. (C.12) as

$$
r_{\mathrm{L}}(k)=\frac{\alpha \beta}{\zeta(\alpha-\beta)}\left((\zeta-\alpha) \mathrm{e}^{\alpha z}+(\beta-\zeta) \mathrm{e}^{\beta z}\right) \Theta(-z),
$$

and $s_{\mathrm{L}}(z)$ for negative $z$ is derived by:

$$
\begin{aligned}
s_{\mathrm{L}}(z) & =r_{\mathrm{L}}(z)-\mathrm{i} \operatorname{Res}_{\mathrm{i} \alpha}[T(k)] \frac{R_{\mathrm{L}}(\mathrm{i} \alpha)}{T(\mathrm{i} \alpha)} \mathrm{e}^{\alpha z}-\mathrm{i} \operatorname{Res}_{\mathrm{i} \beta}[T(k)] \frac{R_{\mathrm{L}}(\mathrm{i} \beta)}{T(\mathrm{i} \beta)} \mathrm{e}^{\beta z} \\
& =0, \quad z<0 .
\end{aligned}
$$

Inserting this result into the left Marchenko equation for $z=x+y<0$ yields the trivial solution

$$
A_{\mathrm{L}}(x, x)=0, \quad x<0 .
$$

With Eq. (C.17), the corresponding result for the potential is

$$
U_{2}(x)=0, \quad x<0 .
$$


For $x=0$, this procedure yields no result. But the $1 / k$-dependency of $R_{2}(k)$ for large $k$ leads to a delta function contribution in the potential [117], and thus

$$
U_{2}(x)=c_{0} \delta(x)-\sigma^{2} \cosh ^{-2}(\sigma x+\phi / 2) \Theta(x)
$$

with an unknown amplitude $c_{0}$. It can be determined by the matching conditions of the eigenfunctions at $x=0$. For the potential $U_{2}(x)$ of Eq. (5.113), explicit eigenfunctions $\varphi_{k}(x)$ which satisfy the stationary Schrödinger equation

$$
-\varphi_{k}^{\prime \prime}(x)+2 U_{2}(x) \varphi_{k}(x)=k^{2} \varphi_{k}(x)
$$

are given by [118]

$$
\varphi_{k}(x)=\frac{1}{\sqrt{2 \pi}} \begin{cases}\mathrm{e}^{\mathrm{i} k x}+R_{2}(k) \mathrm{e}^{-\mathrm{i} k x}, & x<0 \\ T_{2}(k) \frac{k+\mathrm{i} \sigma \tanh (\sigma x+\phi / 2)}{k+\mathrm{i} \sigma} \mathrm{e}^{\mathrm{i} k x}, & x>0 .\end{cases}
$$

The matching condition for $\varphi_{k}(x)$ at $x=0$ reads

$$
1+R_{2}(k)=T_{2}(k) \frac{k+\mathrm{i} \sigma \tanh (\phi / 2)}{k+\mathrm{i} \sigma}
$$

and it provides a useful check for the derivation of $U_{2}(x)$. After some algebra, Eq. (5.116) can be shown to hold, using $\tanh (\phi / 2)=-(\alpha+\beta-\alpha \beta / \zeta) / \sigma$ with Eq. (5.107).

From the matching condition for the first derivative at $x=0$, the remaining unknown $c_{0}$ can be determined, since $R_{2}(k)$ and $T_{2}(k)$ are known by Eqs. (5.94) and (5.100). This matching condition reads

$$
\varphi_{k}^{\prime}(0+)-\varphi_{k}^{\prime}(0-)=2 c_{0} \varphi_{k}(0)
$$

and some lengthy calculation yields the result

$$
c_{0}=-\frac{\alpha \beta}{\zeta} .
$$

Thus, the final expression for the second order filter potential is

$$
U_{2}(x)=-\frac{\alpha \beta}{\zeta} \delta(x)-\sigma^{2} \cosh ^{-2}(\sigma x+\phi / 2) \Theta(x) .
$$

This potential is shown in Fig. 5.7 without the Dirac delta contribution.

As a further test for the validity of the derivation, the reflection coefficient for incidence from the left and for the potential $U_{2}(x)$ is calculated numerically by means of the method provided in Section B.1.4, where the delta potential in $U_{2}(x)$ is approximated by a Gaussian function $(\sqrt{2 \pi} \epsilon)^{-1} \exp \left(-x^{2} / 2 \epsilon^{2}\right)$ with $\epsilon=0.01$. It is shown in Fig. 5.8, together with the original reflection coefficient $R_{2}(k)$. The coincidence is perfect, thus validating the present result for $U_{2}(x)$. Moreover, this example demonstrates that the delta potential, obtained mathematically as an ideal construct can be understood physically in terms of a very narrow Gaussian, whose width is small comparing to all other length scales of the model. 


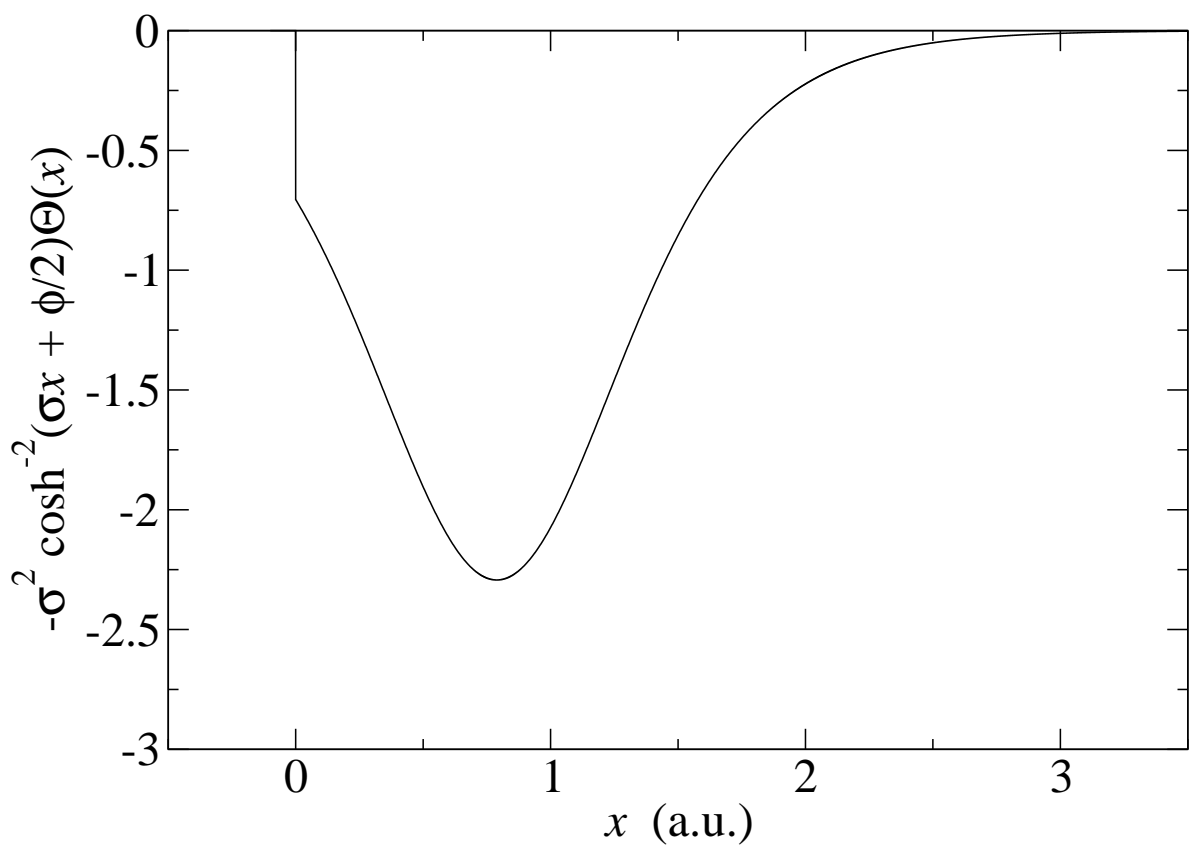

Figure 5.7: Real-valued second order filter potential $U_{2}(x)$ of Eq. (5.119) without the Dirac delta distribution; $k_{0}=0.42$.

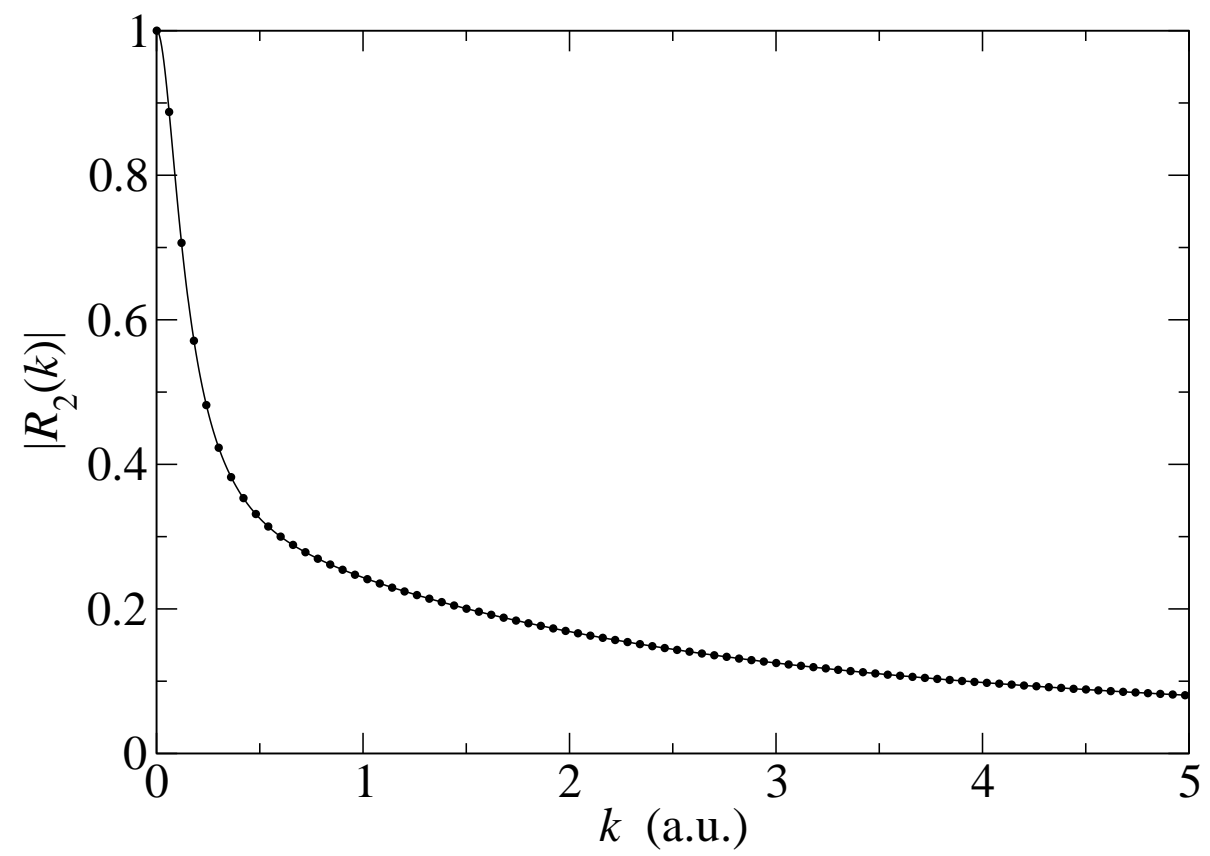

Figure 5.8: Comparison between $\left|R_{2}(k)\right|$ (solid line) and a numerical derivation of the reflection coefficient for the potential $U_{2}(x)$ (circles), $k_{0}=0.042$. The delta potential in $U_{2}(x)$ is approximated by the Gaussian function $(\sqrt{2 \pi} \epsilon)^{-1} \exp \left(-x^{2} / 2 \epsilon^{2}\right)$ with $\epsilon=0.01$. 


\section{Chapter 6}

\section{Arrival times in the presence of interactions}

It has been pointed out in Section 2.1.5 that some of the fundamental problems concerning the concept of arrival times for free motion may be regarded as solved by the concept of POVMs and the related arrival-time distribution of Kijowski. However, the original papers of Kijowski [10] and Giannitrapani [11] addressed only free particles. Since then some efforts have been undertaken to generalize free arrivaltime distributions to the case of particles interacting with an external potential [4].

An intuitive approach is the use of the quantum mechanical flux as an arrivaltime distribution since it can be easily generalized to the interaction case. This has been investigated in the literature $[57,62,119,63,46]$, but of course, the drawback related to the backflow effect mentioned in Section 2.1.3 remains. In contrast, León et al. proposed a generalization of the Aharonov-Bohm time operator by means of a quantum canonical transformation [50] and the authors derived an arrivaltime distribution which is related to Kijowski's distribution but it is not bilinear in the state. With a definition of an arrival-time distribution that is based on the norm of the quantum state and which is different from the flux or from Kijowski's distribution, Aoki et al. [120] studied tunneling particles and compared their method with the stochastic interpretation of quantum mechanics introduced by Nelson [121]. Several proposals for a generalization of Kijowski's arrival-time distribution have been put forward by Brouard et al. by means of the "crossing state" concept [25].

In this chapter, the new approach to quantum arrival times obtained in this work which is based on an operational model and on operator normalization as a mathematical tool is applied to particles interacting with an external potential or coming in from both sides [122]. For the interaction case, a new formula which generalizes Kijowski distribution is derived and it is investigated for some particular potentials. For the case of free particles coming in from both sides, Eq. (2.28) is recovered. It shall be emphasized that the generalized arrival-time distribution derived in this chapter is the first one that is based on an operational measurement procedure. 


\subsection{Operator-normalized absorption rate}

Throughout this chapter, the one-channel limit of Section 3.4.1 is assumed to be valid, i.e. one works in a regime where $\gamma \rightarrow \infty$ with $\Omega^{2} / \gamma=$ const. holds. It has been shown that the operational distribution of the time of the first photon emission in that case equals the absorption rate of the imaginary potential $V(x)=-\mathrm{i} \hbar \Omega^{2}(x) / 2 \gamma$, for $\delta=0$, i.e. the resonant case. In the following, a very narrow imaginary potential is used as a "detector",

$$
V(x)=-\mathrm{i} V_{\epsilon} \chi_{\epsilon}(x),
$$

where $\chi_{\epsilon} \equiv \chi_{[-\epsilon, \epsilon]}$, and the relation to the laser model is given by $V_{\epsilon}=\hbar \Omega^{2} / 2 \gamma$. Ideally, one intends to take the limit $\epsilon \rightarrow 0$ at the end, where two situations are distinguished:

(a) $V_{\epsilon}=V_{0} L_{0} / \epsilon$

(b) $V_{\epsilon}=V_{0}\left(L_{0} / \epsilon\right)^{\alpha}, 0<\alpha<1$.

Case (a) yields an imaginary delta-function potential, whereas case (b) implies a weaker and less perturbing measurement. The latter case is preferred in the following but the analysis of the first one is included for completeness and comparison. The corresponding limits are denoted by $\epsilon \stackrel{\text { (a) }}{\longrightarrow} 0$ and $\epsilon \stackrel{(\mathrm{b})}{\longrightarrow} 0$, respectively. For particular calculations, $\alpha=1 / 2$ will be chosen.

Additional to the considerations in the previous chapter, the approach is now generalized for particles interacting with some external potential $U(x)$, where $U(x)$ is assumed to vanish sufficiently fast for $|x| \rightarrow \infty$ to be in line with usual scattering situations [116]. The conditional Hamiltonian of a particle with mass $m$ interacting with $U(x)$ and the measurement potential $V(x)$ reads

$$
H_{\mathrm{c}}=\frac{\widehat{p}^{2}}{2 m}+U(\widehat{x})+V(\widehat{x}) .
$$

To obtain the time development under $H_{\mathrm{c}}$ of a wave packet which is asymptotically free, one first solves the stationary equation

$$
H_{\mathrm{c}} \phi_{k}=E_{k} \phi_{k}
$$

for scattering states with real energy $E_{k}=\hbar^{2} k^{2} / 2 m$. For later purpose, the eigenstates of the Hamiltonian in the absence of the measurement potential, $\varphi_{k}$, are defined by

$$
\left(\frac{\widehat{p}^{2}}{2 m}+U(\widehat{x})\right) \varphi_{k}=E_{k} \varphi_{k}
$$

The time development of an initial state is obtained by its decomposition into a superposition of eigenfunctions of $H_{\mathrm{c}}$. This is easy for an initial free wave packet coming in from $x=-\infty$ in the remote past. Indeed,

$$
\psi(x, t)=\int_{0}^{\infty} \mathrm{d} k \widetilde{\psi}(k) \phi_{k}(x) e^{-\mathrm{i} \hbar k^{2} t / 2 m}
$$


describes the time development of a state which in the remote past behaves like a free wave packet whose momentum amplitude at $t=0$ is $\widetilde{\psi}(k), k>0$. Here, $\phi_{k}$ corresponds to the scattering states for left incidence. In the absence of a real potential, $U=0$, states with both positive and negative momenta are considered as well. Symmetrical and antisymmetrical wave components can be treated in a similar way, as shown later in more detail.

As shown in Section 5.1, it is convenient to use the time development operator in the interaction picture with respect to $H_{0}=\widehat{p}^{2} / 2 m$,

$$
U_{\mathrm{c}}^{\mathrm{I}}\left(t, t_{0}\right)=\mathrm{e}^{\mathrm{i} H_{0} t / \hbar} \mathrm{e}^{-\mathrm{i} H_{\mathrm{c}}\left(t-t_{0}\right) / \hbar} \mathrm{e}^{-\mathrm{i} H_{0} t_{0} / \hbar}
$$

Then, Eq. (6.5) can be written as

$$
\left|\psi_{t}\right\rangle=\mathrm{e}^{-\mathrm{i} H_{0} t / \hbar} U_{\mathrm{c}}^{\mathrm{I}}(t,-\infty)|\psi\rangle
$$

where $|\psi\rangle=\int \mathrm{d} k \widetilde{\psi}(k)|k\rangle$. The probability density of no detection until time $t$ is given by

$$
N(t)=\left\|\psi_{t}\right\|^{2}=\left\langle\psi\left|U_{\mathrm{c}}^{\mathrm{I}}(t,-\infty)^{\dagger} U_{\mathrm{c}}^{\mathrm{I}}(t,-\infty)\right| \psi\right\rangle,
$$

and the corresponding operator is

$$
\widehat{N}_{t}=U_{\mathrm{c}}^{\mathrm{I}}(t,-\infty)^{\dagger} U_{\mathrm{c}}^{\mathrm{I}}(t,-\infty) .
$$

The probability density for the first detection to occur at $t$ reads

$$
\begin{aligned}
\Pi(t) & =-\frac{\mathrm{d}}{\mathrm{d} t} N(t)=\frac{2 V_{\epsilon}}{\hbar} \int_{-\epsilon}^{\epsilon} \mathrm{d} x|\psi(x, t)|^{2} \\
& =\int_{0}^{\infty} \mathrm{d} k \mathrm{~d} k^{\prime} \overline{\widetilde{\psi}(k)} \widetilde{\psi}\left(k^{\prime}\right) \mathrm{e}^{\mathrm{i} \hbar\left(k^{2}-k^{\prime 2}\right) t / 2 m} f_{\epsilon}\left(k, k^{\prime}\right),
\end{aligned}
$$

with the kernel function

$$
f_{\epsilon}\left(k, k^{\prime}\right)=\frac{2 V_{\epsilon}}{\hbar} \int_{-\epsilon}^{\epsilon} \mathrm{d} x \overline{\phi_{k}(x)} \phi_{k^{\prime}}(x)
$$

According to previous results, this absorption rate corresponds to the temporal distribution of the first spontaneously emitted photon upon interaction with the laser region and it models the arrival-time probability density. $\Pi(t)$ can be written as an expectation value of the incoming states, $\Pi(t)=\left\langle\psi\left|\widehat{\Pi}_{t}\right| \psi\right\rangle$, with an operator

$$
\widehat{\Pi}_{t}=\frac{2 V_{\epsilon}}{\hbar} U_{\mathrm{c}}^{\mathrm{I}}(t,-\infty)^{\dagger} \chi_{\epsilon}(\widehat{x}) U_{\mathrm{c}}^{\mathrm{I}}(t,-\infty) .
$$

Now, $\Pi(t)$ can be normalized on the level of operators as shown in Section 5.1 by defining a normalization operator $\widehat{B}$ on the incoming states,

$$
\widehat{B}=\int_{-\infty}^{\infty} \mathrm{d} t \widehat{\Pi}_{t}=\widehat{1}-\widehat{N}_{\infty}
$$


and an operator-normalized distribution by

$$
\begin{aligned}
\Pi^{\mathrm{ON}}(t) & =\left\langle\psi\left|\widehat{\Pi}_{t}^{\mathrm{ON}}\right| \psi\right\rangle \\
& =\left\langle\psi\left|\widehat{B}^{-1 / 2} \widehat{\Pi}_{t} \widehat{B}^{-1 / 2}\right| \psi\right\rangle
\end{aligned}
$$

Eq. (6.14) can be evaluated in $k$-space, since it is known from Section 5.1 that $\left\langle k|\widehat{B}| k^{\prime}\right\rangle=b\left(k, k^{\prime}\right) \delta\left(k-k^{\prime}\right)$, and $b\left(k, k^{\prime}\right)$ has to cancel the factors which arise from integrating $\Pi(t)$ over $t$, see Section 5.1.5. This leads to

$$
b(k, k)^{-1 / 2}=\left(\frac{\hbar k}{2 \pi m f_{\epsilon}(k, k)}\right)^{1 / 2}
$$

and Eq. (6.14) becomes

$$
\Pi^{\mathrm{ON}}(t)=\frac{\hbar}{2 \pi m} \int_{0}^{\infty} \mathrm{d} k \mathrm{~d} k^{\prime} \overline{\widetilde{\psi}(k)} \widetilde{\psi}\left(k^{\prime}\right) \mathrm{e}^{\mathrm{i} \hbar\left(k^{2}-k^{\prime 2}\right) t / 2 m} \sqrt{k k^{\prime}} \frac{f_{\epsilon}\left(k, k^{\prime}\right)}{\sqrt{f_{\epsilon}(k, k) f_{\epsilon}\left(k^{\prime}, k^{\prime}\right)}} .
$$

This expression has the form of a modified Kijowski distribution, where all the information on the potential $U(x)$ and the measurement are contained in the function

$$
F_{\epsilon}\left(k, k^{\prime}\right)=\frac{f_{\epsilon}\left(k, k^{\prime}\right)}{\sqrt{f_{\epsilon}(k, k) f_{\epsilon}\left(k^{\prime}, k^{\prime}\right)}}
$$

$F_{\epsilon}\left(k, k^{\prime}\right)$ is investigated in the following within the limits $\epsilon \stackrel{(\mathrm{a})}{\longrightarrow} 0, \epsilon \stackrel{(\mathrm{b})}{\longrightarrow} 0$.

\subsection{Generalized free arrival-time distributions}

In the case of free arrival times one has

$$
U(x)=0,
$$

and the solution of Eq. (6.3) in the presence of the imaginary potential $-\mathrm{i} V_{\epsilon}$ is given by

$$
\phi_{k}^{[i]}(x)=\frac{1}{\sqrt{2 \pi}}\left(A_{i} \mathrm{e}^{\mathrm{i} k_{i} x}+B_{i} \mathrm{e}^{-\mathrm{i} k_{i} x}\right)
$$

for the three regions $i, i=0,1,2$ associated with $x \leq-\epsilon,-\epsilon \leq x \leq \epsilon, \epsilon \leq x$, respectively, where $k_{0}=k_{2} \equiv k$ and

$$
k_{1} \equiv q_{\epsilon}=\sqrt{k^{2}+\mathrm{i} m V_{\epsilon} / \hbar^{2}} .
$$

The amplitudes $A_{i}$ and $B_{i}$ are determined by the matching conditions at $x=-\epsilon$ and $x=\epsilon$. They are given in Section B.1 of the Appendix. 


\subsubsection{Left incidence}

For left incoming states, the appropriate eigenstates have boundary conditions $A_{0}=$ 1 and $B_{2}=0$. Then one can solve Eq. (B.8) for the other amplitudes, with $\mathbf{T}(0,2)$ given in Eq. (B.11), to obtain

$$
\begin{aligned}
& A_{2}=\left[T_{11}(0,2)\right]^{-1}=\mathrm{e}^{-2 \mathrm{i} k \epsilon} / D, \\
& B_{0}=T_{21}(0,2) A_{2}=\frac{\mathrm{i}}{2}\left(\frac{q_{\epsilon}}{k}-\frac{k}{q_{\epsilon}}\right) \sin \left(2 q_{\epsilon} \epsilon\right) \mathrm{e}^{-2 \mathrm{i} k \epsilon} / D, \\
& A_{1}=T_{11}(1,2) A_{2}=\frac{1}{2}\left(1+\frac{k}{q_{\epsilon}}\right) \mathrm{e}^{-\mathrm{i}\left(k+q_{\epsilon}\right) \epsilon} / D, \\
& B_{1}=T_{21}(1,2) A_{2}=\frac{1}{2}\left(1-\frac{k}{q_{\epsilon}}\right) \mathrm{e}^{-\mathrm{i}\left(k-q_{\epsilon}\right) \epsilon} / D,
\end{aligned}
$$

with the common denominator

$$
D=\cos \left(2 q_{\epsilon} \epsilon\right)-\frac{\mathrm{i}}{2}\left(\frac{q_{\epsilon}}{k}+\frac{k}{q_{\epsilon}}\right) \sin \left(2 q_{\epsilon} \epsilon\right) .
$$

In the limit $\epsilon \stackrel{(a)}{\longrightarrow} 0$ one has

$$
A_{1}, B_{1} \rightarrow \frac{1}{2} \frac{\hbar^{2} k}{\hbar^{2} k+m V_{0} L_{0}}, \quad \epsilon \stackrel{(\mathrm{a})}{\longrightarrow} 0,
$$

which is also obtained by considering a delta potential from the beginning. With Eq. (6.19) it follows that

$$
f_{\epsilon}\left(k, k^{\prime}\right)=\int_{-\epsilon}^{\epsilon} \mathrm{d} x \overline{\phi_{k}^{[1]}(x)} \phi_{k^{\prime}}^{[1]}(x) \sim \frac{4 \epsilon}{\pi} A_{1}(k) A_{1}\left(k^{\prime}\right), \quad \epsilon \stackrel{(\mathrm{a})}{\longrightarrow} 0,
$$

and since this expression is real, one has

$$
F_{\epsilon}\left(k, k^{\prime}\right) \rightarrow 1, \quad \epsilon \stackrel{(\mathrm{a})}{\longrightarrow} 0,
$$

and Eq. (6.16) yields

$$
\Pi^{\mathrm{ON}}(t) \rightarrow \Pi_{\mathrm{K}}(t), \quad \epsilon \stackrel{(\mathrm{a})}{\longrightarrow} 0 .
$$

The same result holds for the "weak" limit $\epsilon \stackrel{(\mathrm{b})}{\longrightarrow} 0$, since here

$$
A_{1}, B_{1} \rightarrow \frac{1}{2}, \quad \epsilon \stackrel{(\mathrm{b})}{\longrightarrow} 0,
$$

which leads again to Eq. (6.25) and finally to

$$
\Pi^{\mathrm{ON}}(t) \rightarrow \Pi_{\mathrm{K}}(t), \quad \epsilon \stackrel{(\mathrm{b})}{\longrightarrow} 0 .
$$

In principle, these results are not new, because in Section 5.2 a similar result has been obtained for the laser model. In contrast, the symmetric or antisymmetric incidence of a superposition of wave packets coming in from opposite sides to the arrival point $x_{\mathrm{A}}=0$ is a much more interesting case and shall be investigated in the following. 


\subsubsection{Symmetric and antisymmetric incidence}

For symmetric (antisymmetric) incidence, the corresponding eigenstates of Eq. (6.3) are denoted by $\phi_{k}^{\mathrm{s}}(x)\left(\phi_{k}^{\mathrm{a}}(x)\right)$ and they are obtained with the boundary conditions $A_{0}=1$ and $B_{2}=1\left(B_{2}=-1\right)$. As before, $k>0$ holds. Using the transfer matrix method of Appendix B.1, the amplitudes inside the measurement region for the symmetric case are given by

$$
\begin{aligned}
A_{1}=B_{1} & =\frac{\mathrm{e}^{-\mathrm{i} k \epsilon}}{\cos \left(q_{\epsilon} \epsilon\right)-\frac{\mathrm{i} q_{\epsilon}}{k} \sin \left(q_{\epsilon} \epsilon\right)}, \quad \text { (symmetric incidence) } \\
& \rightarrow \frac{\hbar^{2} k}{\hbar^{2} k+m V_{0} L_{0}}, \quad \epsilon \stackrel{(\mathrm{a})}{\longrightarrow} 0 \\
& \rightarrow 1, \quad \epsilon \stackrel{(\mathrm{b})}{\longrightarrow} 0,
\end{aligned}
$$

whereas for the antisymmetric case one has

$$
\begin{aligned}
A_{1}=-B_{1} & =\frac{\mathrm{e}^{-\mathrm{i} k \epsilon}}{\frac{q_{\epsilon}}{k} \cos \left(q_{\epsilon} \epsilon\right)-\mathrm{i} \sin \left(q_{\epsilon} \epsilon\right)}, \quad \text { (antisymmetric incidence) } \\
& \rightarrow \frac{\hbar k \epsilon^{1 / 2}}{\sqrt{\mathrm{i} m V_{0} L_{0}}}, \quad \epsilon \stackrel{(\mathrm{a})}{\longrightarrow} 0, \\
& \rightarrow \frac{\hbar k \epsilon^{\alpha / 2}}{\sqrt{\mathrm{i} m V_{0} L_{0}}}, \quad \epsilon \stackrel{(\mathrm{b})}{\longrightarrow} 0 .
\end{aligned}
$$

Inserting these amplitudes into $\phi_{k}^{\mathrm{s}}(x)$ and $\phi_{k}^{\mathrm{a}}(x)$ for the region $i=1$, respectively, yields with Eqs. (6.19) and (6.11) and for the symmetric case

$$
f_{\epsilon}^{\mathrm{s}}\left(k, k^{\prime}\right)=\int_{-\epsilon}^{\epsilon} \mathrm{d} x \overline{\phi_{k}^{s,[1]}(x)} \phi_{k^{\prime}}^{s,[1]}(x) \sim \frac{4 \epsilon}{\pi} A_{1}(k) A_{1}\left(k^{\prime}\right), \quad \epsilon \stackrel{(\mathrm{a})}{\longrightarrow} 0, \epsilon \stackrel{(\mathrm{b})}{\longrightarrow} 0,
$$

and thus

$$
F_{\epsilon}^{\mathrm{s}}\left(k, k^{\prime}\right) \rightarrow 1, \quad \epsilon \stackrel{(\mathrm{a})}{\longrightarrow} 0, \epsilon \stackrel{(\mathrm{b})}{\longrightarrow} 0 .
$$

The operator-normalized arrival-time distribution for a symmetric wave packet $\left|\psi_{\mathrm{s}}\right\rangle$ reads

$$
\Pi_{\mathrm{s}}^{\mathrm{ON}}(t) \rightarrow \frac{\hbar}{2 \pi m}\left|\int_{0}^{\infty} \mathrm{d} k \widetilde{\psi}_{\mathrm{s}}(k) \sqrt{k} \mathrm{e}^{-\mathrm{i} \hbar k^{2} t / 2 m}\right|^{2}, \quad \epsilon \rightarrow 0
$$

For the antisymmetric case the situation is more complicated, since the eigenstates $\phi_{k}^{\mathrm{a}}(x)$ vanish at $x=0$ and so does the first order expansion of $f_{\epsilon}^{\mathrm{a}}\left(k, k^{\prime}\right)$ for $\epsilon \rightarrow 0$. But a more precise investigation gives a non-vanishing contribution in the next order:

$$
\begin{aligned}
f_{\epsilon}^{\mathrm{a}}\left(k, k^{\prime}\right) & =\int_{-\epsilon}^{\epsilon} \mathrm{d} x \overline{\phi_{k}^{a,[1]}(x)} \phi_{k^{\prime}}^{a,[1]}(x) \\
& \sim \frac{\hbar^{2} k k^{\prime} \epsilon^{\alpha}}{2 \pi m V_{0} L_{0}^{\alpha}} \int_{-\epsilon}^{\epsilon} \mathrm{d} x\left(\mathrm{e}^{-\mathrm{i} \bar{q}_{\epsilon} x}-\mathrm{e}^{\mathrm{i} \bar{q}_{\epsilon} x}\right)\left(\mathrm{e}^{\mathrm{i} q_{\epsilon}^{\prime} x}-\mathrm{e}^{-\mathrm{i} q_{\epsilon}^{\prime} x}\right) \\
& \sim \frac{2 \hbar^{2} k k^{\prime} \epsilon^{\alpha}}{\pi m V_{0} L_{0}^{\alpha}}\left(\frac{\sin \left[\left(\bar{q}_{\epsilon}-q_{\epsilon}^{\prime}\right) \epsilon\right]}{\bar{q}_{\epsilon}-q_{\epsilon}^{\prime}}-\frac{\sin \left[\left(\bar{q}_{\epsilon}+q_{\epsilon}^{\prime}\right) \epsilon\right]}{\bar{q}_{\epsilon}+q_{\epsilon}^{\prime}}\right) \\
& \sim \frac{4}{3 \pi} k k^{\prime} \epsilon^{3}, \quad \epsilon \stackrel{(\mathrm{a})}{\longrightarrow} 0, \epsilon \stackrel{(\mathrm{b})}{\longrightarrow} 0 .
\end{aligned}
$$


With Eq. (6.17) it follows that

$$
F_{\epsilon}^{\mathrm{a}}\left(k, k^{\prime}\right) \rightarrow 1, \quad \epsilon \stackrel{(\mathrm{a})}{\longrightarrow} 0, \epsilon \stackrel{(\mathrm{b})}{\longrightarrow} 0
$$

In spite of the fact that an antisymmetric wave function $\left|\psi_{\mathrm{a}}\right\rangle$ vanishes at $x=0$, the operator normalization preserves a finite arrival time distribution. With Eq. (6.16), the result is the same as for the symmetric case, namely Kijowski's distribution,

$$
\Pi_{\mathrm{a}}^{\mathrm{ON}}(t) \rightarrow \frac{\hbar}{2 \pi m}\left|\int_{0}^{\infty} \mathrm{d} k \widetilde{\psi}_{\mathrm{a}}(k) \sqrt{k} \mathrm{e}^{-\mathrm{i} \hbar k^{2} t / 2 m}\right|^{2}, \quad \epsilon \rightarrow 0 .
$$

\subsubsection{General incidence}

An arbitrary wave packet $\psi(x, t)$ can be decomposed as a superposition of symmetric and antisymmetric states,

$$
|\psi\rangle=\frac{1}{\sqrt{2}}\left(\left|\psi_{\mathrm{s}}\right\rangle+\left|\psi_{\mathrm{a}}\right\rangle\right)
$$

Now, the operator-normalized arrival-time distribution for an arbitrary wave packet is the sum of four terms:

$$
\Pi^{\mathrm{ON}}(t)=\left\langle\psi\left|\widehat{\Pi}_{t}^{\mathrm{ON}}\right| \psi\right\rangle=\frac{1}{2} \sum_{\substack{i=\mathrm{s}, \mathrm{a} \\ j=\mathrm{s}, \mathrm{a}}}\left\langle\psi_{i}\left|\widehat{\Pi}_{t}^{\mathrm{ON}}\right| \psi_{j}\right\rangle .
$$

The cross terms in Eq. (6.38) vanish, because the Hamiltonian in Eq. (6.2) commutes with the parity operator, $\widehat{\pi}$, for $U=0$,

$$
\left[H_{\mathrm{c}}, \widehat{\pi}\right]=0, \quad \text { for } U=0 .
$$

As a consequence, the operators $H_{\mathrm{c}}^{\mathrm{I}}, U_{\mathrm{c}}^{\mathrm{I}}, \widehat{B}$ and $\widehat{\Pi}_{t}^{\mathrm{ON}}$ also commute with $\widehat{\pi}$. Therefore the operational arrival-time distribution for a general wave packet in the limit $\epsilon \rightarrow 0$ is the sum of Kijowski's distribution for the symmetric and the antisymmetric part,

$$
\begin{aligned}
\Pi^{\mathrm{ON}}(t) & =\frac{1}{2}\left(\Pi_{\mathrm{s}}^{\mathrm{ON}}(t)+\Pi_{\mathrm{a}}^{\mathrm{ON}}(t)\right) \\
& \rightarrow \frac{\hbar}{4 \pi m}\left(\left|\int_{0}^{\infty} \mathrm{d} k \widetilde{\psi}_{\mathrm{s}}(k) \sqrt{k} \mathrm{e}^{-\mathrm{i} \hbar k^{2} t / 2 m}\right|^{2}+\left|\int_{0}^{\infty} \mathrm{d} k \widetilde{\psi}_{\mathrm{a}}(k) \sqrt{k} \mathrm{e}^{-\mathrm{i} \hbar k^{2} t / 2 m}\right|^{2}\right)
\end{aligned}
$$

This result can be written in a more concise style by noting that

$$
\begin{aligned}
& \widetilde{\psi}_{\mathrm{s}}(k)=\frac{1}{\sqrt{2}}(\widetilde{\psi}(k)+\widetilde{\psi}(-k)), \\
& \widetilde{\psi}_{\mathrm{a}}(k)=\frac{1}{\sqrt{2}}(\widetilde{\psi}(k)-\widetilde{\psi}(-k)),
\end{aligned}
$$


which yields the following expression after expanding the absolute values in Eq. (6.40),

$$
\Pi^{\mathrm{ON}}(t) \rightarrow \frac{\hbar}{2 \pi m} \sum_{\alpha= \pm}\left|\int_{0}^{\infty} \mathrm{d} k \widetilde{\psi}(\alpha k) \sqrt{k} \mathrm{e}^{-\mathrm{i} \hbar k^{2} t / 2 m}\right|^{2}, \quad \epsilon \rightarrow 0 .
$$

In this form, the generalized expression for the free arrival-time distribution of an arbitrary wave packet coincides with Eq. (2.28) and with previous proposals which are based more on heuristic arguments [4]. Clearly, the operator-normalized distribution shows no interferences between positive and negative momentum components, a point that has been extensively discussed in the literature [4].

\subsection{Generalized arrival-time distribution in the presence of interactions}

In the presence of an external interaction the complete Hamiltonian (6.2) is considered without assuming Eq. (6.18). For example, the potential $U(x)$ can be experimentally realized by a strongly detuned laser field as shown in Section 3.4.1 or by vacuum induced potentials [123, 124].

In the following, the derivation is performed within the limit $\epsilon \stackrel{(b)}{\longrightarrow} 0$ for the measurement potential. This corresponds to a non-disturbing measurement, since the transfer matrix connecting the left and the right border of the measurement potential $V(x)$ is given in that limit by $\frac{1}{2}\left(\begin{array}{ll}1 & 0 \\ 0 & 1\end{array}\right)$, according to Eq. (B.11) with $\kappa=q_{\epsilon}$ and $l=2 \epsilon, s=0$. This means that the wave function inside the measurement potential equals in the limit $\epsilon \stackrel{(\mathrm{b})}{\longrightarrow} 0$ the wave function at the arrival point in the absence of any absorbing potential, $\varphi_{k}(x=0)$. With the assumption that $\phi_{k}(x)$ does not vanish identically at the arrival point $x=0$ (this excludes the case of antisymmetric wave packets considered in the previous section), Eq. (6.11) becomes

$$
f_{\epsilon}\left(k, k^{\prime}\right) \sim \frac{2 V_{0} L_{0}^{\alpha}}{\hbar \epsilon^{\alpha-1}} \overline{\varphi_{k}(0)} \varphi_{k^{\prime}}(0), \quad \epsilon \stackrel{(\mathrm{b})}{\longrightarrow} 0
$$

and Eq. (6.17) leads to

$$
F_{\epsilon}\left(k, k^{\prime}\right)=\frac{\overline{\varphi_{k}(0)}}{\left|\varphi_{k}(0)\right|} \frac{\varphi_{k^{\prime}}(0)}{\left|\varphi_{k^{\prime}}(0)\right|}=\exp \left[-\mathrm{i} \arg \left(\varphi_{k}(0)-\varphi_{k^{\prime}}(0)\right)\right], \quad \epsilon \stackrel{(\mathrm{b})}{\longrightarrow} 0 .
$$

Inserting this result into Eq. (6.16), one obtains the generalization of Kijowski's arrival-time distribution, valid for arbitrary external interaction potentials and wave packets in the sense of the above conditions,

$$
\Pi^{\mathrm{ON}}(t) \rightarrow \Pi_{\mathrm{K}, \mathrm{gen}}(t)=\frac{\hbar}{2 \pi m}\left|\int_{0}^{\infty} \mathrm{d} k \widetilde{\psi}(k) \mathrm{e}^{-\mathrm{i} \hbar k^{2} t / 2 m} \sqrt{k} \mathrm{e}^{\mathrm{i} \arg \varphi_{k}(0)}\right|^{2}, \quad \epsilon \stackrel{(\mathrm{b})}{\longrightarrow} 0 .
$$

Note that one obtains the arrival-time distribution at an arbitrary point $x_{\mathrm{A}}$ by shifting the absorbing potential, i.e. by replacing $[-\epsilon, \epsilon]$ by $\left[-\epsilon+x_{\mathrm{A}}, \epsilon+x_{\mathrm{A}}\right]$ in 
Eq. (6.1) in which case the result reads

$$
\Pi_{\mathrm{K}, \text { gen }}\left(t, x_{\mathrm{A}}\right)=\frac{\hbar}{2 \pi m}\left|\int_{0}^{\infty} \mathrm{d} k \widetilde{\psi}(k) \mathrm{e}^{-\mathrm{i} \hbar k^{2} t / 2 m} \sqrt{k} \mathrm{e}^{\mathrm{i} \arg \varphi_{k}\left(x_{\mathrm{A}}\right)}\right|^{2} .
$$

This is one of the most important results of this work. Obviously, $\Pi_{\mathrm{K}, \operatorname{gen}}\left(t, x_{\mathrm{A}}\right)$ is a positive, normalized distribution that can be written as a bilinear form of the state. This latter property distinguishes $\Pi_{\mathrm{K}, \mathrm{gen}}(t)$ from previous attempts of generalizations. The case of free motion is included, since $\arg \varphi_{k}(x)=k x$ for $U=0$ and thus Eq. (2.14) arises.

The mean arrival time for the distribution (6.47) and for a Gaussian wave packet given by Eq. (2.19) is

$$
\begin{aligned}
\langle t\rangle & =\int_{-\infty}^{\infty} \mathrm{d} t \Pi_{\mathrm{K}, \text { gen }}(t) t \\
& =\frac{m}{\hbar} \int_{0}^{\infty} \mathrm{d} k|\widetilde{\psi}(k)|^{2} \frac{\left|x_{0}\right|+\frac{\partial}{\partial k} \arg \varphi_{k}\left(x_{\mathrm{A}}\right)}{k},
\end{aligned}
$$

where $x_{0}<0$ denotes the mean position of the left-incoming wave packet at $t=0^{1}$.

In the following subsections, $\Pi_{\mathrm{K}, \mathrm{gen}}\left(t, x_{\mathrm{A}}\right)$ and $\langle t\rangle$ are investigated for various scattering situations. For $x_{\mathrm{A}}=0$, the position-dependency in the arrival-time distribution is omitted, $\Pi_{\mathrm{K}, \text { gen }}(t) \equiv \Pi_{\mathrm{K} \text {,gen }}(t, 0)$. It shall be emphasized that the description of arrival times inside the potential region do not cause any problems in the present approach, since the absorbing potential can be placed in an arbitrary region.

\subsection{Example: Rectangular barrier}

\subsubsection{Arrival time behind a potential barrier}

The wave function behind a rectangular potential barrier located in the region $a \leq$ $x \leq b<0$ with height $U$ and length $l=b-a$ for an initial plane wave coming in from the left and in the absence of any imaginary potential is given by

$$
\varphi_{k}(x)=\frac{1}{\sqrt{2 \pi}} T(k) \mathrm{e}^{\mathrm{i} k x}, \quad x \geq b
$$

where the transmission amplitude $T(k)$ results from the matching conditions and it is given by $A_{2}$ in Eq. (B.12) of the appendix,

$$
T(k)=\frac{\mathrm{e}^{-\mathrm{i} k l}}{\cos (\kappa l)-\frac{\mathrm{i}}{2}\left(\frac{\kappa}{k}+\frac{k}{\kappa}\right) \sin (\kappa l)},
$$

with

$$
\kappa=\sqrt{k^{2}-2 m U / \hbar^{2}}
$$

\footnotetext{
${ }^{1}$ For arbitrary wave packets, $x_{0}$ has to be replaced by $-\hbar \operatorname{Im}\left(\frac{\mathrm{d}}{\mathrm{d} k} \widetilde{\psi}(k) / \widetilde{\psi}(k)\right)[125]$.
} 


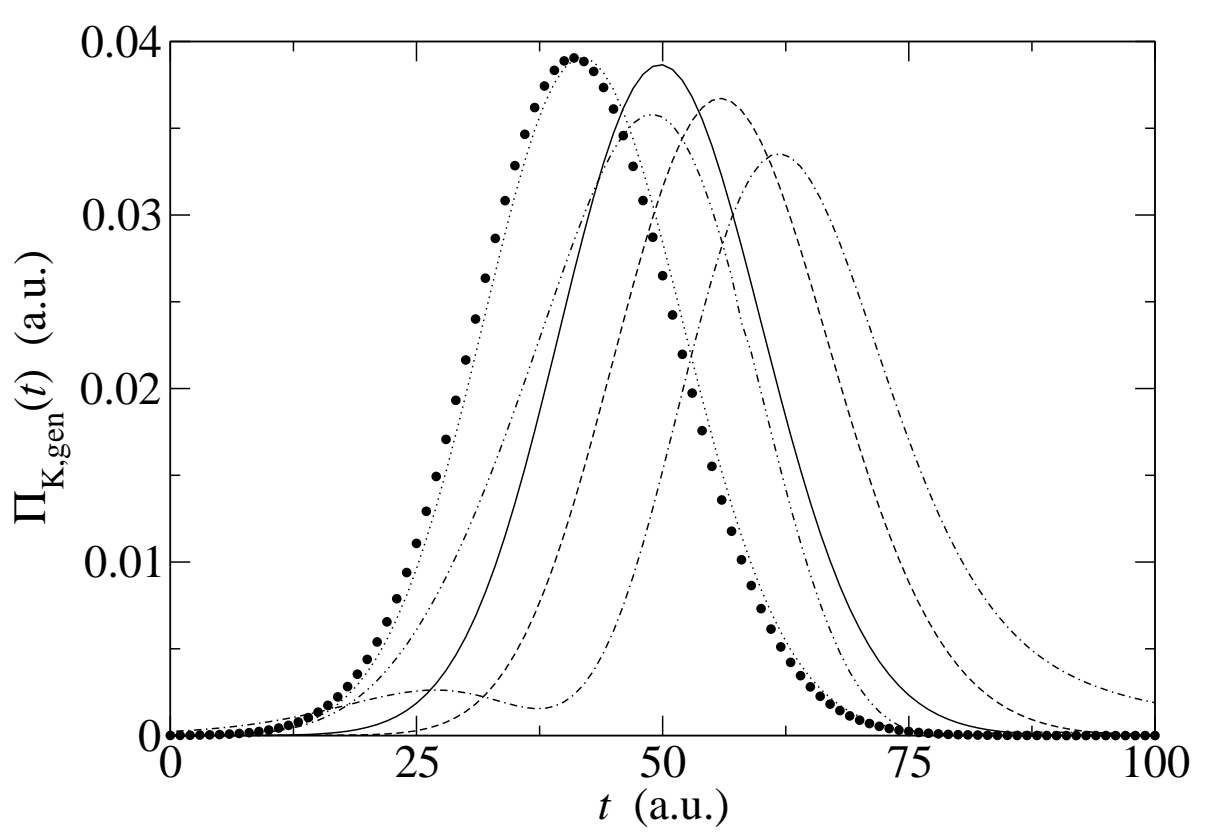

Figure 6.1: Arrival-time distribution $\Pi_{\mathrm{K}, \mathrm{gen}}(t)$ at $x=0$ behind a potential barrier with height $U=0$ (solid), $U=0.3$ (dashed), $U=0.48$ (dot-dashed), $U=0.58$ (dot-dot-dashed), $U=1.0$ (dotted), $U=2.0$ (circles), potential width $l=10$, for a minimum-uncertainty Gaussian wave packet at $t=0\left(x_{0}=-50, \Delta x=10, v_{0}=1\right)$. For large $U$, the mean arrival time approaches the Hartman time.

Thus, the generalized arrival-time distribution for arrivals at $x_{\mathrm{A}}=0$ behind a barrier is given with Eq. (6.46) as

$$
\Pi_{\mathrm{K}, \text { gen }}(t)=\frac{\hbar}{2 \pi m}\left|\int_{0}^{\infty} \mathrm{d} k \widetilde{\psi}(k) \mathrm{e}^{-\mathrm{i} \hbar k^{2} t / 2 m} \sqrt{k} \mathrm{e}^{\mathrm{i} \arg T(k)}\right|^{2} .
$$

A numerical example for this distribution is given in Fig. 6.1 for various barrier heights $U$ and fixed potential width $l=10$ in atomic units. It is visible that for increasing $U$ the arrival time at $x_{\mathrm{A}}=0$ of a particle starting at time $t=0$ and position $\langle x\rangle=x_{0}<0$ with mean velocity $\langle v\rangle=v_{0}$ is first delayed, but when the potential strength is further enhanced, an asymptotic distribution is reached with a mean arrival time ("Hartman time") $t_{\mathrm{H}}=\left(\left|x_{0}\right|-l\right)\left\langle v^{-1}\right\rangle$ which is smaller than the free arrival time $\left|x_{0}\right|\left\langle v^{-1}\right\rangle$. This phenomenon is related to the Hartman effect [18] which can indeed be observed [54] and is discussed in detail in the following section.

Obviously, the arrival-time distribution for tunneling particles differs from previous proposals, in particular from the one of León et al. [50]. Nevertheless, the new approach of this work which uses an operator-normalized absorption rate yields the result of Ref. [50] after some modifications. This is explained in the following.

First relation to Ref. [50] In the above setup an incoming free particle was prepared far to the left and it then interacts with an external real potential. A different situation arises when one includes the real potential as part of the preparation procedure through which the particle passes far away on the left and then continues to 
propagate freely. In this case the incident state for operator normalization purposes would be the normalized transmitted wave packet. Formally, this packet may be formed by a projection onto a large region to the right of the real potential. For the positive results of this projection, i.e. transmissions, the normalized incoming free state is then characterized by $T(k) \widetilde{\psi}(k) /\left(\int \mathrm{d} k|T(k) \widetilde{\psi}(k)|^{2}\right)^{1 / 2}$ instead of $\widetilde{\psi}(k)$. Applying Kijowski's distribution to the incoming free state thus prepared gives an expression that is referred to as León's distribution in the following, since it agrees with the result of Ref. [50],

$$
\Pi_{\mathrm{L}}(t)=\frac{\hbar}{2 \pi m}\left|\int_{0}^{\infty} \mathrm{d} k \widetilde{\psi}(k) T(k) \mathrm{e}^{-\mathrm{i} \hbar k^{2} t / 2 m} \sqrt{k}\right|^{2}\left(\int_{0}^{\infty} \mathrm{d} k|T(k) \widetilde{\psi}(k)|^{2}\right)^{-1} .
$$

It is thus seen to be related to $\Pi_{\mathrm{K} \text {,gen }}(t)$ by a state preparation procedure that selects the transmitted particles. In contrast to $\Pi_{\mathrm{K}, \text { gen }}(t)$, the expression $\Pi_{\mathrm{L}}(t)$ is no bilinear form of the state $|\psi\rangle$.

Second relation to Ref. [50] A second connection can be established by the choice of a different normalization constant for the operator normalization. The transmission probability through the rectangular potential barrier is given by the expression $\int \mathrm{d} k|\widetilde{\psi}(k) T(k)|^{2}$ and one may argue that in this case the total arrivaltime probability should not equal 1 but should equal this transmission probability. Instead of $\Pi^{\mathrm{ON}}(t)$ one would then have a modified distribution, $\Pi_{\mathrm{mod}}^{\mathrm{ON}}(t)$, satisfying

$$
\int_{-\infty}^{\infty} \mathrm{d} t \Pi_{\bmod }^{\mathrm{ON}}(t)=\int_{0}^{\infty} \mathrm{d} k|\widetilde{\psi}(k) T(k)|^{2}
$$

In terms of operators this would require an operator, $\widehat{\Pi}_{t, \text { mod }}^{\text {ON }}$, satisfying

$$
\int_{-\infty}^{\infty} \mathrm{d} t \widehat{\Pi}_{t, \text { mod }}^{\mathrm{ON}}=\int_{0}^{\infty} \mathrm{d} k|T(k)|^{2}|k\rangle\langle k|
$$

Since with Eq. (6.44) one has

$$
f_{\epsilon}\left(k, k^{\prime}\right) \sim \overline{\varphi_{k}(0)} \varphi_{k^{\prime}}(0) \sim \overline{T(k)} T\left(k^{\prime}\right), \quad \epsilon \stackrel{(\mathrm{b})}{\longrightarrow} 0,
$$

the modified kernel of the normalization operator $\widehat{B}^{-1 / 2}$ shall not cancel the contributions of the transmission coefficients. Thus, Eq. (6.15) has to be modified, such that

$$
b(k, k)^{-1 / 2}=\sqrt{\hbar k /(2 \pi m)},
$$

which yields instead of Eq. (6.16)

$$
\Pi_{\text {mod }}^{\mathrm{ON}}(t) \sim \int_{0}^{\infty} \mathrm{d} k \mathrm{~d} k^{\prime} \bar{\psi}(k) \widetilde{\psi}\left(k^{\prime}\right) \mathrm{e}^{\mathrm{i} \hbar\left(k^{2}-k^{\prime 2}\right) t / 2 m} \sqrt{k k^{\prime}} f_{\epsilon}\left(k, k^{\prime}\right) .
$$

The modified arrival-time distribution in the limit $\epsilon \stackrel{(\mathrm{b})}{\longrightarrow} 0$ finally reads

$$
\Pi_{\text {mod }}^{\mathrm{ON}}(t) \rightarrow \frac{\hbar}{2 \pi m}\left|\int_{0}^{\infty} \mathrm{d} k \widetilde{\psi}(k) e^{-\mathrm{i} \hbar k^{2} t / 2 m} \sqrt{k} T(k)\right|^{2}, \quad \epsilon \stackrel{(\mathrm{b})}{\longrightarrow} 0,
$$


which satisfies Eq. (6.54) and gives the joint probability density for both arrival and transmission. Normalizing this to 1 by an ordinary normalization just yields the distribution $\Pi_{\mathrm{L}}(t)$ of Eq. (6.53). Therefore $\Pi_{\mathrm{L}}(t)$ can be understood as a conditional probability density for the arrival time of the particle under the condition that it has been transmitted through the potential barrier. In contrast, such an interpretation is not possible for the new distribution $\Pi_{\mathrm{K} \text {,gen }}$ derived in this work, since it includes the corresponding fraction on the level of operators and before taking expectation values.

\subsubsection{Mean arrival times and the Hartman effect}

To compare the presented operational approach to tunneling times with previous works, it is useful to consider not only the arrival-time distribution but also mean arrival times. The analysis is carried out in the following for the example of a wave packet that comes from the far left and collides with a rectangular potential barrier at $a \leq x \leq b<0$. The arrival time behind the barrier is measured at $x_{\mathrm{A}}=0$.

The mean arrival time of the distribution $\Pi_{\mathrm{K}, \mathrm{gen}}$ in Eq. (6.52) is given by Eq. (6.48) and Eq. (6.49) and it reads for a Gaussian packet

$$
\langle t\rangle=\frac{m}{\hbar} \int_{0}^{\infty} \mathrm{d} k|\widetilde{\psi}(k)|^{2} \frac{\left|x_{0}\right|+\frac{\partial}{\partial k} \arg T(k)}{k},
$$

where $x_{0}<0$ denotes the value for the mean position of the wave packet at $t=0$.

This result for $\langle t\rangle$ can be understood as the average of the "phase times", i.e. the time required for a freely moving particle plus Wigner's time delay of Eq. (2.39), over the initial state. In contrast, previous proposals for the mean arrival time are written in terms of an average over the transmitted state [50, 120], which is just the first moment of the arrival-time distribution $\Pi_{\mathrm{L}}(t)$ of Eq. (6.53). It is denoted by $\langle t\rangle_{\mathrm{L}}$ in the following and given explicitly by

$$
\langle t\rangle_{\mathrm{L}}=\frac{m}{\hbar} \int_{0}^{\infty} \mathrm{d} k|T(k) \widetilde{\psi}(k)|^{2} \frac{\left|x_{0}\right|+\frac{\partial}{\partial k} \arg T(k)}{k}\left(\int_{0}^{\infty} \mathrm{d} k|T(k) \widetilde{\psi}(k)|^{2}\right)^{-1} .
$$

These results are not contradictory but correspond to different state preparations, as explained in the previous section. Note that $\langle t\rangle_{\mathrm{L}}$ agrees with the arrival time defined by the tunneled flux in Eq. (2.42).

The dependence of $\langle t\rangle$ with the potential height is shown in Fig. 6.2 where $\langle t\rangle$ is plotted versus $U$ for a fixed barrier width $l$. In the free limit $U \rightarrow 0,\langle t\rangle$ tends to an "averaged free arrival time", $\left|x_{0}\right|\left\langle v^{-1}\right\rangle$, since $T(k)$ approaches to 1 . For $U \rightarrow \infty$ one has with Eq. (6.50)

$$
\arg T(k) \sim-k l-\pi / 2, \quad U \rightarrow \infty,
$$

and $\langle t\rangle$ approaches the Hartman time $t_{\mathrm{H}}=\left(\left|x_{0}\right|-l\right)\left\langle v^{-1}\right\rangle$, where $\left\langle v^{-1}\right\rangle$ is given by $\left\langle v^{-1}\right\rangle=\int \mathrm{d} k|\widetilde{\psi}(k)|^{2} m / \hbar k$.

For analyzing the dependence of the mean tunneling time with the barrier width $l$, a "mean tunneling time" or traversal time $\tau_{\mathrm{T}}$ is heuristically defined as an easily 


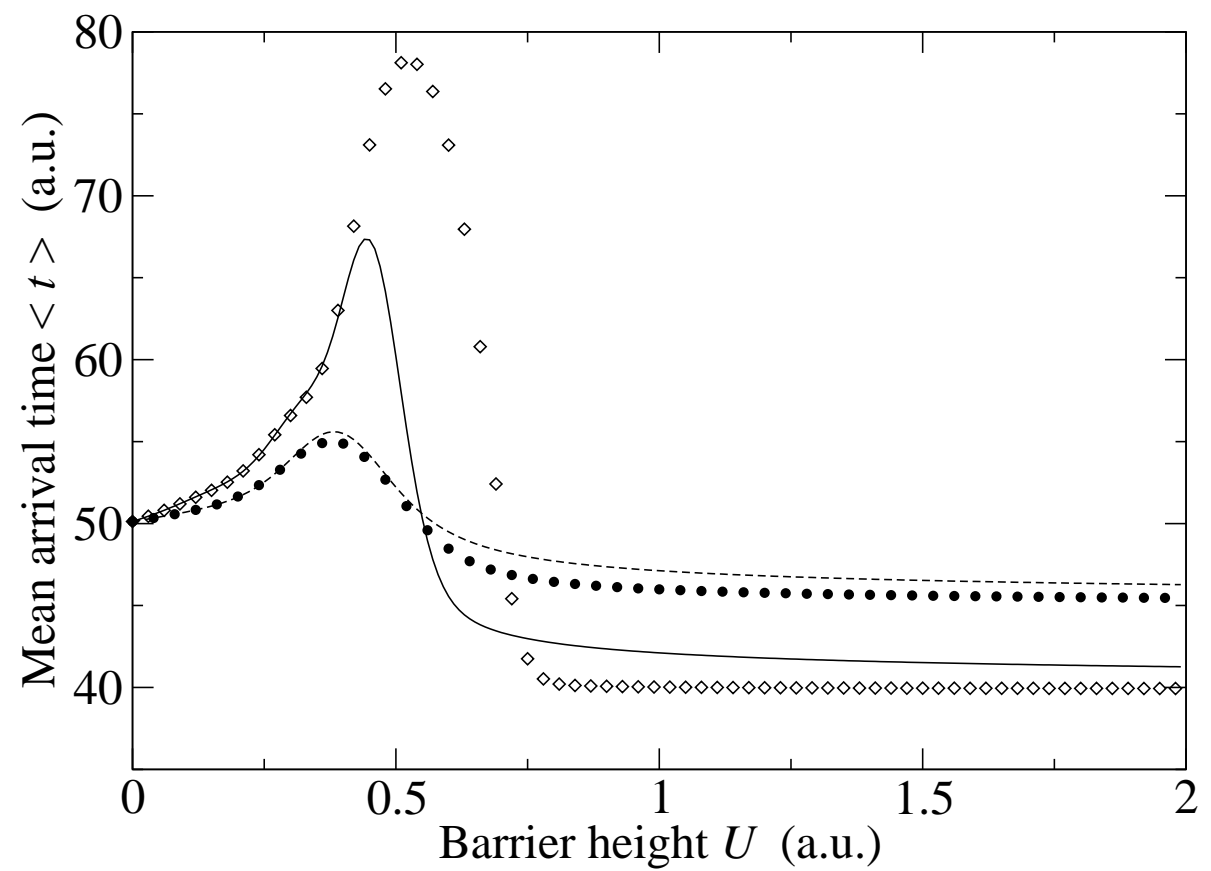

Figure 6.2: The mean arrival time $\langle t\rangle$ of Eq. (6.48) behind a barrier of fixed width $l=10$ (solid line), $l=5$ (dashed line) as a function of the barrier height $U$. The wave packet at $t=0$ is the same minimum-uncertainty Gaussian as in Fig. 6.1. For comparison, the arrival time $\langle t\rangle_{\mathrm{L}}$ of Eq. (6.61) is shown for the same parameter values, $l=10$ (diamonds), $l=5$ (filled circles). For $U \rightarrow 0$, one has the free arrival time $\left|x_{0}\right|\left\langle v^{-1}\right\rangle$, and for $U \rightarrow \infty$ the arrival time approaches the Hartman time $t_{\mathrm{H}}=\left(\left|x_{0}\right|-l\right)\left\langle v^{-1}\right\rangle$.

calculable quantity by subtracting the classical time for crossing the non-potential region with average momentum $k_{0}=m v_{0} / \hbar$ from $\langle t\rangle$ [122],

$$
\tau_{\mathrm{T}}=\langle t\rangle-\frac{m\left(\left|x_{0}\right|-l\right)}{\hbar k_{0}}
$$

It has been pointed out that these "extrapolated phase times" for traversal should not be over-interpreted as actual traversal times [13], but they are useful for a comparison with other results. In a tunneling collision of a quantum particle with an opaque square barrier, the Hartman effect [18] is the near independence of $\tau_{\mathrm{T}}$ from the barrier width $l$ over a wide regime. It has been recently explained as a saturation effect of the integrated probability density under the barrier [126]. An experimental verification of the Hartman effect has been reported in Refs. [54, 17] by means of photon tunneling. There exist bounds for the negative scattering time delay due to causality [127, 125].

The occurrence of the Hartman effect is shown in Fig. 6.3, where the tunneling time $\tau_{\mathrm{T}}$ is plotted versus the potential width $l$. For thin barriers, $\tau_{\mathrm{T}}$ is larger than the free traversal time, as shown in the inset, but for increasing $l$ there is a sudden transition from a positive delay to a negative one for increasing $U$. It is clearly visible that, in the negative delay regime, the tunneling time is nearly constant for 


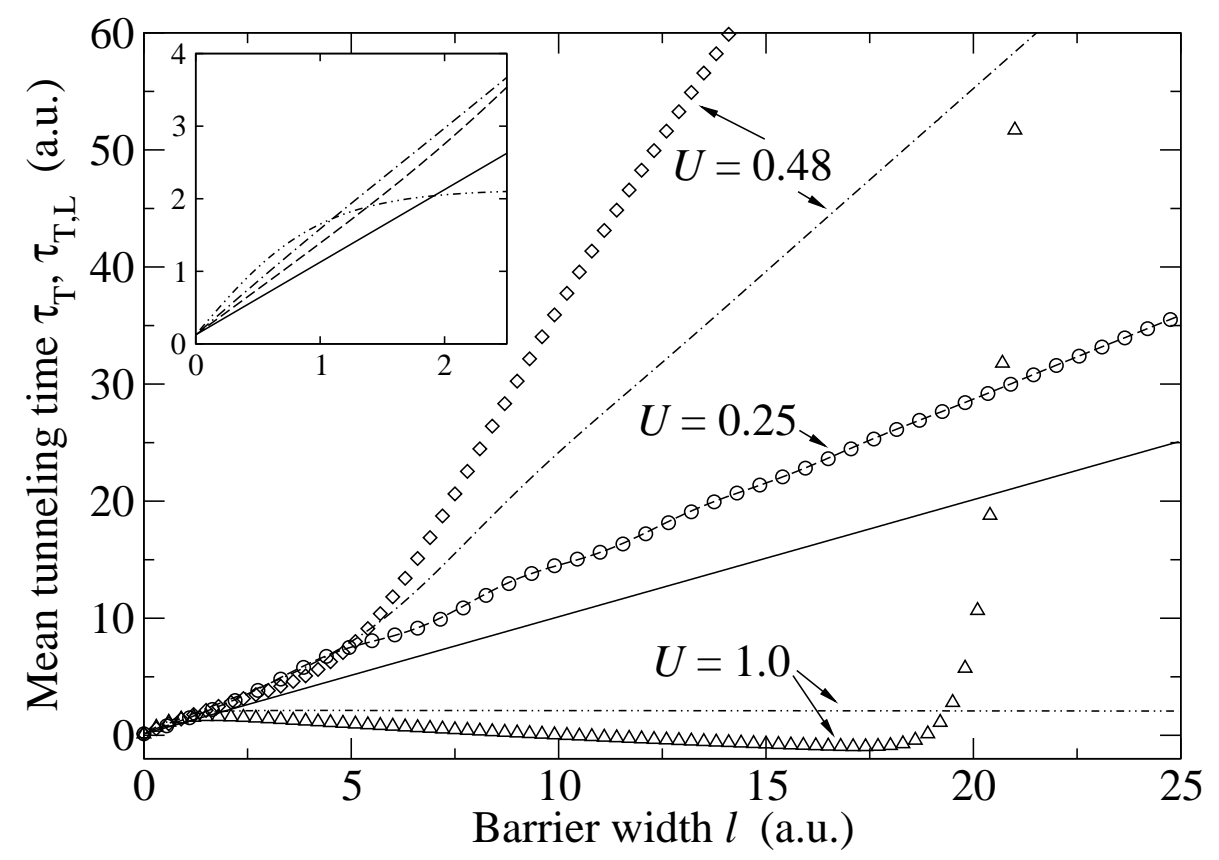

Figure 6.3: Mean tunneling time $\tau_{\mathrm{T}}$ (lines) of Eq. (6.63) versus $l$ compared with the tunneling time $\tau_{\mathrm{T}, \mathrm{L}}$ (symbols) of Eq. (6.64) for different barrier heights $U=0$ (solid), $U=0.25$ (dashed, circles), $U=0.48$ (dot-dashed, diamonds) and $U=1.0$ (dot-dot-dashed, triangles) in atomic units. The wave packet at $t=0$ is the same minimum-uncertainty Gaussian packet as in Fig. 6.1.

increasing $l$ (Hartman regime). For comparison, the tunneling time with respect to the arrival-time distribution $\Pi_{\mathrm{L}}(t)$ of Eq. (6.53) is plotted and denoted by $\tau_{\mathrm{T}, \mathrm{L}}$,

$$
\tau_{\mathrm{T}, \mathrm{L}}=\langle t\rangle_{\mathrm{L}}-\frac{m\left(\left|x_{0}\right|-l\right)}{\hbar k_{0}}
$$

The behavior of $\tau_{\mathrm{T}, \mathrm{L}}$ for thin barriers is similar, but for increasing $l$ the tunneling time derived with $\Pi_{\mathrm{L}}(t)$ first slowly decreases and gets actually negative [63, 13]. The point one has to emphasize is that for very thick barriers the Hartman effect vanishes for $\tau_{\mathrm{T}, \mathrm{L}}$ which grows linearly for widths larger than a critical barrier length [64]. This is related to the influence of the exponentially decaying $|T(k)|$ in Eq. (6.53), which causes a domination of the above-threshold components of the wave packet [18]. In contrast, $\tau_{\mathrm{T}}$, obtained in this work by means of a different procedure, does not show any transition to the classical-like, ultra-opaque regime. The intuitive explanation is that operator normalization compensates for all detection losses due to the $l$ dependence of $|T(k)|$, so that the result is never dominated by above-the-barrier components. As a consequence, the expression (6.60) does not depend on $|T(k)|$ [122]. 


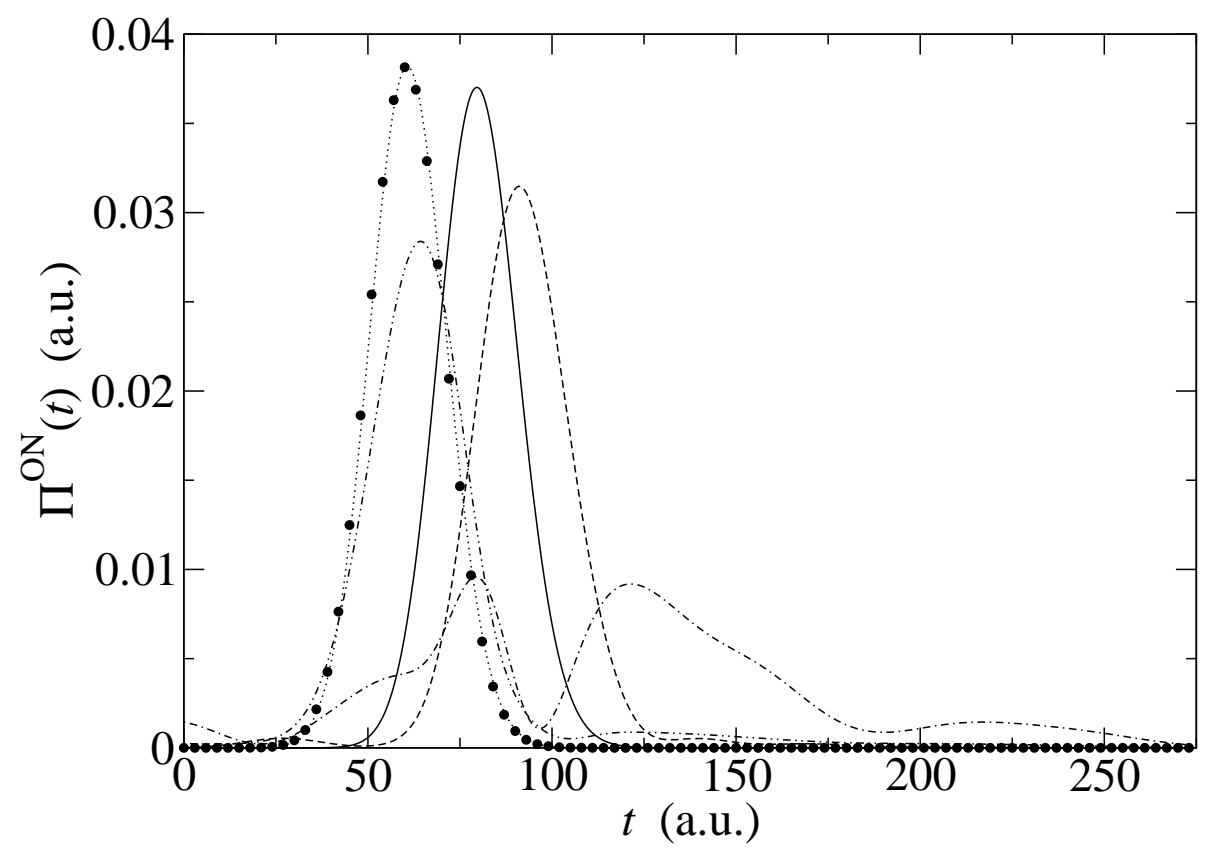

Figure 6.4: Arrival-time distribution $\Pi_{\mathrm{K}, \mathrm{gen}}(t)$ at $x=0$ inside a potential barrier with height $U=0$ (solid), $U=0.3$ (dashed), $U=0.48$ (dot-dashed), $U=0.58$ (dot-dot-dashed), $U=1.0$ (dotted), $U=2.0$ (circles), located between $a=-20$ and $b=20$, for a minimum-uncertainty Gaussian wave packet at $t=0 \quad\left(x_{0}=-80\right.$, $\left.\Delta x=10, v_{0}=1\right)$. For large $U$, the mean arrival time approaches the Hartman time with respect to the crossed distance in the potential.

\subsubsection{Arrival time inside a potential barrier}

To evaluate Eq. (6.46) inside the potential barrier, one needs the wave function $\varphi_{k}(x)$ at $x=0$, where now $a \leq 0 \leq b$. It is given with Eq. (B.3) by

$$
\varphi_{k}(x)=\frac{1}{\sqrt{2 \pi}}\left(A_{1} \mathrm{e}^{\mathrm{i} \kappa x}+B_{1} \mathrm{e}^{-\mathrm{i} k x}\right)
$$

which takes with Eq. (B.12) at $x=0$ the form

$$
\varphi_{k}(0)=\frac{1}{\sqrt{2 \pi}} \mathrm{e}^{\mathrm{i} k b}\left(\cos (\kappa b)-\frac{\mathrm{i} k}{\kappa} \sin (\kappa b)\right) T(k) .
$$

Inserting this expression into Eq. (6.46) yields the generalized Kijowski distribution inside a potential barrier. Clearly, in contrast to the arrival-time distribution behind the potential it explicitly depends on $a$ and $b$, i.e. on the location of the arrival point with respect to the borders. A numerical evaluation of the integral is shown in Fig. 6.4. Similar to the case of arrivals behind the barrier, the distribution is delayed with respect to the free arrival time for small barriers, but for larger barriers (i.e. for barriers which are large compared to the kinetic energy that is associated with the mean momentum of the packet, $U>\hbar^{2}\langle k\rangle^{2} / 2 m$ ) the delay becomes negative and the distribution saturates in the opaque limit. The mean arrival time in this limit is the 
Hartman time with respect to the crossed distance in the potential, $\left(\left|x_{0}\right|-|a|\right)\left\langle v^{-1}\right\rangle$, which is easily seen, since

$$
\arg \varphi_{k}(0) \sim k a-\pi / 2, \quad U \rightarrow \infty
$$

and $a<0$. In other words, the arrival time inside an opaque potential equals the arrival time at the front within the limits of the present approach. Thus, the opaque distance is traversed "instantaneously" - in the sense of wave-packet reshaping. A discussion of causality aspects in this connection can be found in Refs. [127, 125]. Moreover, it is interesting to note that for barrier heights close to the initial mean kinetic energy of the packet the arrival-time distribution exhibits a broad behavior.

One has to emphasize that the present approach to arrival times in the presence of interactions allows the investigation of the Hartman effect inside the potential. It has been shown that not only the mean arrival time $\langle t\rangle$ becomes $l$-independent in the opaque limit, but also the whole arrival-time distribution, which can be seen from Eq. (6.46) and in the Figs. 6.1 and 6.4.

\subsubsection{Arrival time in front of a potential barrier}

Arrival times in front of a potential barrier are particularly interesting, since due to reflections one would expect two clearly separated maxima in the distribution. For the reflected wave packet, the quantum mechanical flux becomes negative. This should not be the case for a true probability distribution as $\Pi_{\mathrm{K} \text {,gen }}$. Arrivals in the presence of partial reflections have been recently investigated by León et al. [50] with a different approach.

According to the method developed in this work, the wave function $\varphi_{k}(x)$ is required at $x=0$ in front of the potential barrier located in the region $0<a \leq x \leq b$. It is given for a left incoming plane wave by Eq. (B.3), where $A_{0}=1$ and the reflection coefficient $B_{0} \equiv R$ is given by Eq. (B.12). This yields

$$
\varphi_{k}(0)=\frac{1}{\sqrt{2 \pi}}(1+R(k))
$$

where

$$
R(k)=\frac{\frac{i}{2}\left(\frac{\kappa}{k}-\frac{k}{\kappa}\right) \sin (\kappa l) \mathrm{e}^{2 i k a}}{\cos (\kappa l)-\frac{i}{2}\left(\frac{\kappa}{k}+\frac{k}{\kappa}\right) \sin (\kappa l)} .
$$

Inserting Eq. (6.68) into Eq. (6.46) yields the arrival-time distribution before a potential barrier. It is numerically evaluated for different barrier heights $U$ and a fixed barrier length $l$ in Fig. 6.5. For comparison, the quantum mechanical flux, $J(t)$, is shown in the inset for the same parameter values. Clearly, $J(t)$ becomes negative for the reflected wave packet.

For free motion arrivals, $U=0$, one has one peak in $\Pi_{\mathrm{K} \text {,gen }}(t)$ centered around $t=\left|x_{0}\right|\left\langle v^{-1}\right\rangle$, as expected. In the presence of partial reflections, a second peak arises at a time that is given approximately by $t=\left(\left|x_{0}\right|+2 a\right)\left\langle v^{-1}\right\rangle$ and it is obviously related to the reflected packet. However, additional peaks are seen for larger times and they become maximal in height for large $U$. This is quite surprising, since these 


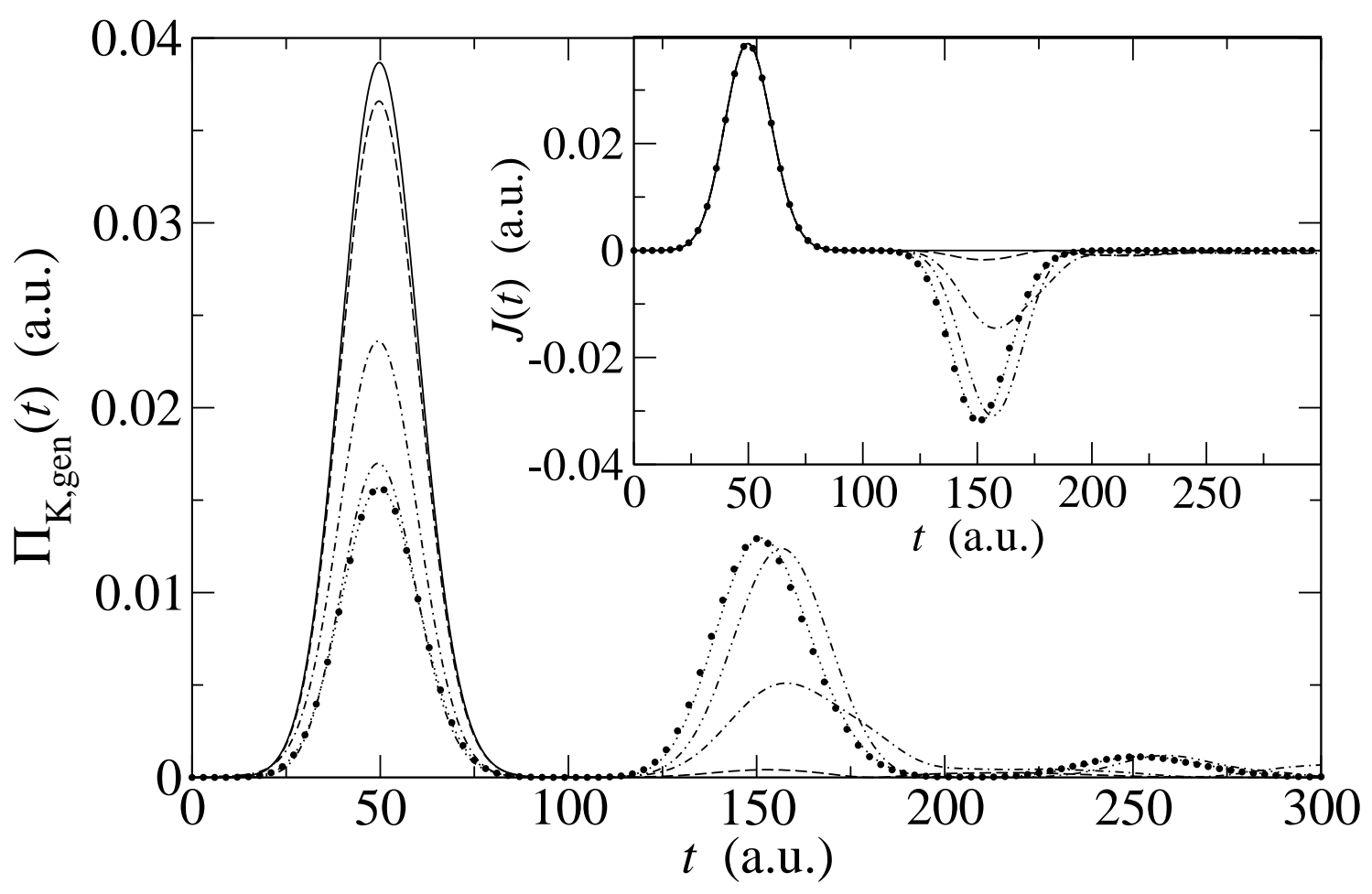

Figure 6.5: arrival-time distribution $\Pi_{\mathrm{K}, \mathrm{gen}}(t)$ at $x=0$ in front of a potential barrier with height $U=0$ (solid), $U=0.3$ (dashed), $U=0.48$ (dot-dashed), $U=0.58$ (dot-dot-dashed), $U=1.0$ (dotted), $U=2.0$ (circles), located between $a=50$ and $b=70$, for a minimum-uncertainty Gaussian wave packet at $t=0 \quad\left(x_{0}=-50\right.$, $\left.\Delta x=10, v_{0}=1\right)$. In the inset, the flux $J(t)$ is shown for the same parameter values for comparison.

peaks for large $U$ do not occur for the flux, as seen from the inset of Fig. 6.5. Thus, they arise through operator normalization. This effect has not been completely understood so far and further research is necessary. A different multi-bump structure for the reflected arrival-time distribution has been found in Ref. [50].

Another interesting behavior can be seen from the second hump in Fig. 6.5. For small $U$, the reflected packet is somewhat delayed with respect to the time that one would classically expect. This is related to the amount of penetration of the packet into the barrier for finite barrier height.

Next one may look at the mean arrival time at $x=0$ in front of the potential barrier. It is given in analogy to Eq. (6.48) and with Eq. (6.68) by

$$
\langle t\rangle=\frac{m}{\hbar} \int_{0}^{\infty} \mathrm{d} k|\widetilde{\psi}(k)|^{2} \frac{\left|x_{0}\right|+\frac{\partial}{\partial k} \arg (1+R(k))}{k} .
$$

For the case of reflection, $\langle t\rangle$ shows a completely different behavior as for transmission. Its dependence from the barrier height $U$ is shown in Fig. 6.6 for different barrier locations. For small barriers, $U \ll m v_{0}^{2} / 2$, the mean arrival time approaches the free arrival time $\left|x_{0}\right|\left\langle v^{-1}\right\rangle$, whereas for large barriers, $U \gg m v_{0}^{2} / 2$, $\langle t\rangle$ approaches the average value of the times for incident and reflected packets, 
$\left[\left|x_{0}\right|\left\langle v^{-1}\right\rangle+\left(\left|x_{0}\right|+2 a\right)\left\langle v^{-1}\right\rangle\right] / 2$. In between, the mean arrival time exhibits jumps at certain values of $U$. This comes from the fact that the argument of $1+R(k)$ is not differentiable for certain values of $k$ and the numerical treatment becomes erroneous in this intermediate regime. Further analytical investigation is necessary to clarify this effect. In contrast to the previous two cases, $\langle t\rangle$ does not become smaller than the free arrival time for arrivals in front of the potential.

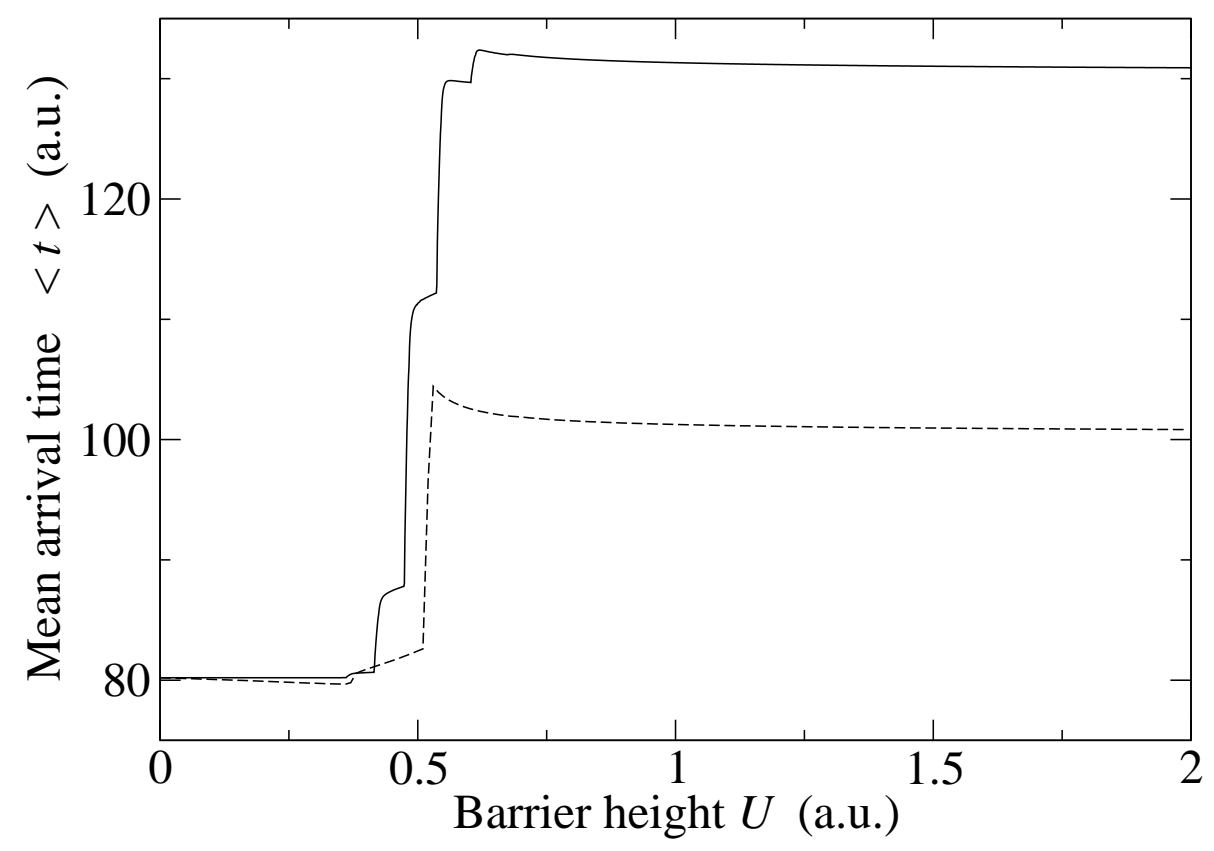

Figure 6.6: The mean arrival time $\langle t\rangle$ of Eq. (6.70) in front of a barrier located between $a=50, b=70$ (solid line) and $a=20, b=70$ (dashed line) as a function of the barrier height $U$. The wave packet at $t=0$ is the same minimum-uncertainty Gaussian state as in Fig. 6.4.

\subsection{Example: Linear potentials}

A different class of potentials to which the present approach to arrival-time distributions may be applied are linear potentials. Their particular importance is related to the fact that they describe particles in the presence of gravitation, as it is usually the fact for cold atoms dropping out of a trap [3] or for present time standards that are based on cesium fountains [128]. Moreover, linear potentials may also be realized by considering ions, interacting with a homogeneous electric field. In the following, one-dimensional scattering situations are solely considered, i.e. the particle is assumed to come in freely from the left, and the positive linear potential applies only for $x \geq 0$. A similar situation has been investigated in Ref. [50], but with a different expression for the mean arrival time. The Hamiltonian in the absence of the imaginary measurement potential reads

$$
H=\frac{\widehat{p}^{2}}{2 m}+f \widehat{x} \Theta(\widehat{x})
$$


where $f>0$ denotes the modulus of the constant force acting on the particle in the right half-space.

The eigenfunctions $\left|\varphi_{k}\right\rangle$ of $H$,

$$
H\left|\varphi_{k}\right\rangle=E_{k}\left|\varphi_{k}\right\rangle, \quad E_{k}=\frac{\hbar^{2} k^{2}}{2 m}
$$

are given for negative $x$ by plane waves incoming from the left,

$$
\varphi_{k}(x)=\frac{1}{\sqrt{2 \pi}}\left(\mathrm{e}^{\mathrm{i} k x}+R(k) \mathrm{e}^{-\mathrm{i} k x}\right), \quad x \leq 0,
$$

whereas for positive $x$ they are solutions of the following differential equation:

$$
\varphi_{k}^{\prime \prime}(x)-F x \varphi_{k}(x)+k^{2} \varphi_{k}(x)=0, \quad x \geq 0,
$$

where $F=2 m f / \hbar^{2}$ has been defined for convenience. The general solution of Eq. (6.74) is given by a superposition of Airy functions [129],

$$
\varphi_{k}(x)=\frac{1}{\sqrt{2 \pi}}\left(C_{1}(k) \operatorname{Ai}\left[F^{-2 / 3}\left(F x-k^{2}\right)\right]+C_{2}(k) \operatorname{Bi}\left[F^{-2 / 3}\left(F x-k^{2}\right)\right]\right), \quad x \geq 0 .
$$

Since $\operatorname{Bi}(x) \rightarrow \infty$ for $x \rightarrow \infty$, one has $C_{2}(k)=0$ for a normalizable wave function. The remaining coefficients $R(k)$ and $C_{1}(k)$ are determined by the matching conditions at $x=0$ and they are given by the following expressions, where $k_{f}=F^{1 / 3}$ and $z_{k}=-k^{2} / k_{f}^{2}$ have been introduced:

$$
\begin{aligned}
R(k) & =\frac{1+\mathrm{i} \frac{k_{f} \mathrm{Ai}^{\prime}\left(z_{k}\right)}{k \mathrm{Ai}\left(z_{k}\right)}}{1-\mathrm{i} \frac{k_{f} \mathrm{Ai}^{\prime}\left(z_{k}\right)}{k \operatorname{Ai}\left(z_{k}\right)}}=\exp \left[2 \mathrm{i} \arctan \left(\frac{k_{f} \mathrm{Ai}^{\prime}\left(z_{k}\right)}{k \operatorname{Ai}\left(z_{k}\right)}\right)\right], \\
C_{1}(k) & =\frac{2}{\operatorname{Ai}\left(z_{k}\right)-\mathrm{i} \frac{k_{f}}{k} \mathrm{Ai}^{\prime}\left(z_{k}\right)} .
\end{aligned}
$$

Since one has full reflection, $|R(k)|=1$ holds. Furthermore, for an infinite force acting on the particle in the right half-space, the case of a hard wall is recovered, and $R \rightarrow-1, C_{1} \rightarrow 0$ for $f \rightarrow \infty$. For later purpose, the phase $\theta(x, k)$ is defined by

$$
\theta(x, k)=\arctan \left(\frac{k_{f} \operatorname{Ai}^{\prime}\left(k_{f} x+z_{k}\right)}{k \operatorname{Ai}\left(k_{f} x+z_{k}\right)}\right) .
$$

Finally, the energy eigenfunctions for the given problem are

$$
\varphi_{k}(x)=\frac{1}{\sqrt{2 \pi}} \begin{cases}\mathrm{e}^{\mathrm{i} k x}+\mathrm{e}^{2 \mathrm{i} \theta(0, k)} \mathrm{e}^{-\mathrm{i} k x}, & x \leq 0 \\ \frac{2 \mathrm{Ai}\left(k_{f} x+z_{k}\right)}{\operatorname{Ai}\left(z_{k}\right)-\mathrm{i} \frac{k_{f}}{k} \mathrm{Ai}^{\prime}\left(z_{k}\right)}, & x \geq 0 .\end{cases}
$$




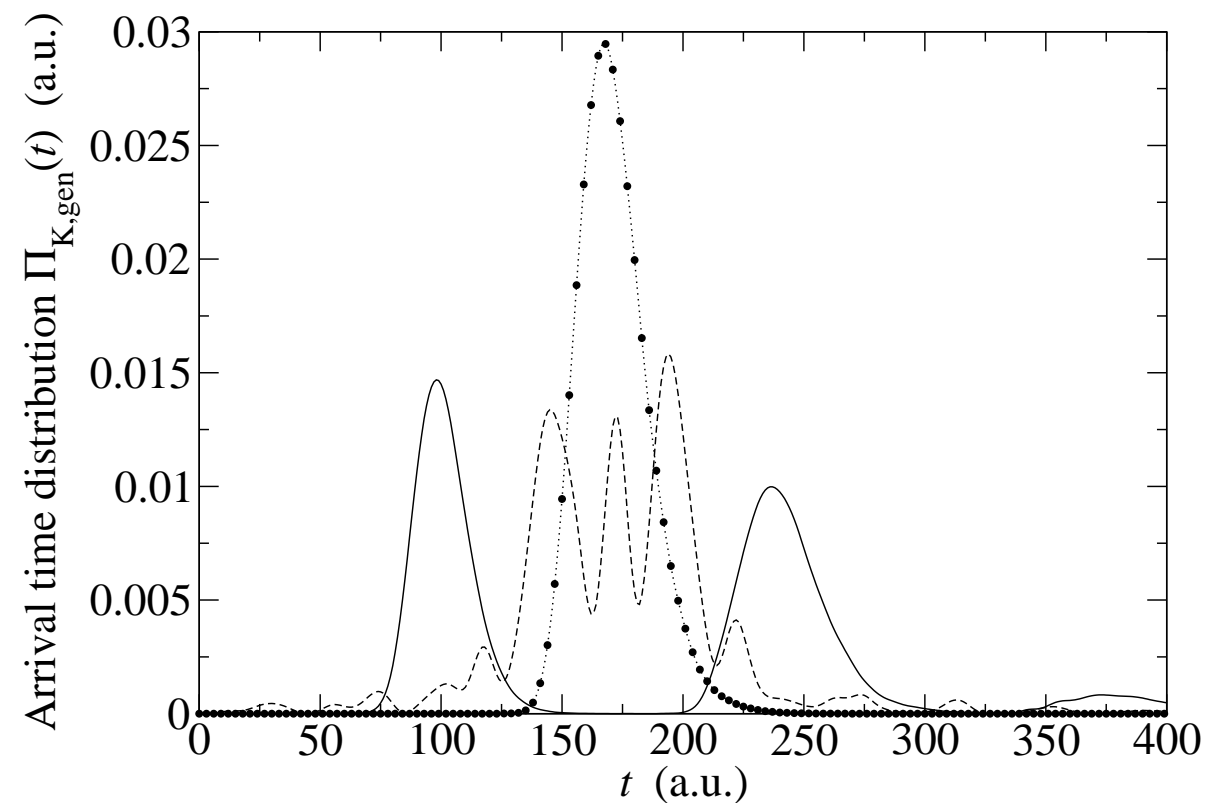

Figure 6.7: Arrival-time distribution in the presence of a linear potential ramp at arrival positions $x_{\mathrm{A}}=-50$ (solid), $x_{\mathrm{A}}=0$ (dashed), $x_{\mathrm{A}}=20$ (dotted) and $x_{\mathrm{A}}=50$ (circles) in atomic units. The wave packet at $t=0$ is a minimum-uncertainty Gaussian state with $x_{0}=-150, v_{0}=1, \Delta x=10$. The steepness of the ramp is given by $F=0.1$, leading to a classical turning point of $x_{\text {turn }}=10$.

This function has to be inserted into Eq. (6.46) to derive the arrival-time distribution. In the case of the linear potential ramp, it is interesting to investigate arrivals at different detector positions $x_{\mathrm{A}}$. This is done by using Eq. (6.47) instead of Eq. (6.46). A numerical example for the arrival-time distribution at different detector positions $x_{\mathrm{A}}$ is shown in Fig. 6.7.

For an arrival position far away from the ramp, one observes two clearly separated peaks in the distribution, related to the incoming and the reflected wave packet. It has been shown that the area under the first two peaks is the same, respectively. Additional small peaks occur similar to the case of reflection at a potential barrier investigated in Section 6.4.4. If the detector is put closer to the classical turning point $x_{\text {turn }}$, given by

$$
\frac{p_{0}^{2}}{2 m}=f x_{\text {turn }}
$$

strong interferences between incoming and reflected packets occur and they are visible in the arrival-time distribution. For detector positions larger than the classical turning point, the two peaks overlap and the distribution saturates. Note that from the arrival-time distribution it is already seen that the mean arrival does not seem to depend on the detector position as it is the case in classical mechanics.

To prove this assumption, the mean arrival time is evaluated in dependence from the detector position $x_{\mathrm{A}}$. It is given by Eq. (6.48). To separate the mean arrival time for the incoming packet from the one for the reflected packet, the solution (6.79) can be written at every point $x$ as a superposition of an incoming and a reflected 
wave with equal amplitudes [50],

$$
\begin{aligned}
& \varphi_{k}(x)=\frac{1}{\sqrt{2 \pi}} \mathrm{e}^{\mathrm{i} \theta(0, k)}\left(\mathrm{e}^{\mathrm{i} k x-\mathrm{i} \theta(0, k)}+\mathrm{e}^{-\mathrm{i} k x+\mathrm{i} \theta(0, k)}\right), x \leq 0 \\
& \varphi_{k}(x)=\frac{1}{\sqrt{2 \pi}} \mathrm{e}^{\mathrm{i} \theta(0, k)} \sqrt{\frac{k^{2} \operatorname{Ai}\left(k_{f} x+z_{k}\right)^{2}+k_{f}^{2} \mathrm{Ai}^{\prime}\left(k_{f} x+z_{k}\right)^{2}}{k^{2} \mathrm{Ai}\left(z_{k}\right)^{2}+k_{f}^{2} \mathrm{Ai}^{\prime}\left(z_{k}\right)^{2}}}\left(\mathrm{e}^{-\mathrm{i} \theta(x, k)}+\mathrm{e}^{\mathrm{i} \theta(x, k)}\right), x \geq 0 .
\end{aligned}
$$

The mean arrival time for the incident and the reflected packet and for detector positions $x_{\mathrm{A}} \leq 0$ reads, respectively,

$$
\begin{aligned}
\langle t\rangle_{\text {in }} & =\frac{m}{\hbar} \int_{0}^{\infty} \mathrm{d} k|\widetilde{\psi}(k)|^{2} \frac{1}{k}\left(\left|x_{0}\right|+x_{\mathrm{A}}\right) \\
\langle t\rangle_{\text {ref }} & =\frac{m}{\hbar} \int_{0}^{\infty} \mathrm{d} k|\widetilde{\psi}(k)|^{2} \frac{1}{k}\left[\left|x_{0}\right|-x_{\mathrm{A}}+2 \frac{\partial}{\partial k} \theta(0, k)\right],
\end{aligned}
$$

whereas for positive detector positions, $x_{\mathrm{A}} \geq 0$, Eq. (6.81) yields

$$
\begin{aligned}
\langle t\rangle_{\text {in }} & =\frac{m}{\hbar} \int_{0}^{\infty} \mathrm{d} k|\widetilde{\psi}(k)|^{2} \frac{1}{k}\left[\left|x_{0}\right|+\frac{\partial}{\partial k}\left(\theta(0, k)-\theta\left(x_{\mathrm{A}}, k\right)\right)\right] \\
\langle t\rangle_{\mathrm{ref}} & =\frac{m}{\hbar} \int_{0}^{\infty} \mathrm{d} k|\widetilde{\psi}(k)|^{2} \frac{1}{k}\left[\left|x_{0}\right|+\frac{\partial}{\partial k}\left(\theta(0, k)+\theta\left(x_{\mathrm{A}}, k\right)\right)\right] .
\end{aligned}
$$

It is easily seen that the average of $\langle t\rangle_{\text {in }}$ and $\langle t\rangle_{\text {ref }}$ is independent of the detector position $x_{\mathrm{A}}$, for the free region as well as for the interaction region. This is the quantum version of the classical result that the sum of the arrival times at $x_{\mathrm{A}}$ of the incoming and the returning particles is twice the arrival time at the turning point, which is independent of $x_{\mathrm{A}}$. An example for the quantum mean arrival time is given in Fig. 6.8, where $\langle t\rangle_{\text {in }}$ and $\langle t\rangle_{\text {ref }}$ are compared with the classical result in dependence of $x_{\mathrm{A}}$. A similar result has been obtained in Ref. [50], but for a different expression for the mean arrival times.

\subsection{Example: Reflectionless potentials}

Another interesting example for studying arrival times in the presence of interaction is given by so-called "transparent" or "reflectionless" potentials which provide a less-known case for analytically solvable models in one dimension. The phase time delay in the presence of those potentials has been investigated in Ref. [127] and a semiclassical derivation of the time advance has been given in Ref. [130]. In this section it is shown that by the methods developed in this work a traversal time can be derived which is in good agreement with both references. In contrast to the previous examples, the reflectionless potentials have bound states, which changes the behavior of the mean arrival time due to the attractive nature of the potential.

Consider the class of one-dimensional potentials given by $U(\xi)=-V_{0} \operatorname{sech}^{2}(b \xi)$ with $V_{0}>0$ and $b \in \mathbb{R}$ where $\operatorname{sech}(x)$ is the inverse hyperbolic cosine function. Clearly, $U(\xi)$ vanishes exponentially for $\xi \rightarrow \pm \infty$ and fulfills the conditions for a standard scattering problem. With dimensionless quantities defined by 


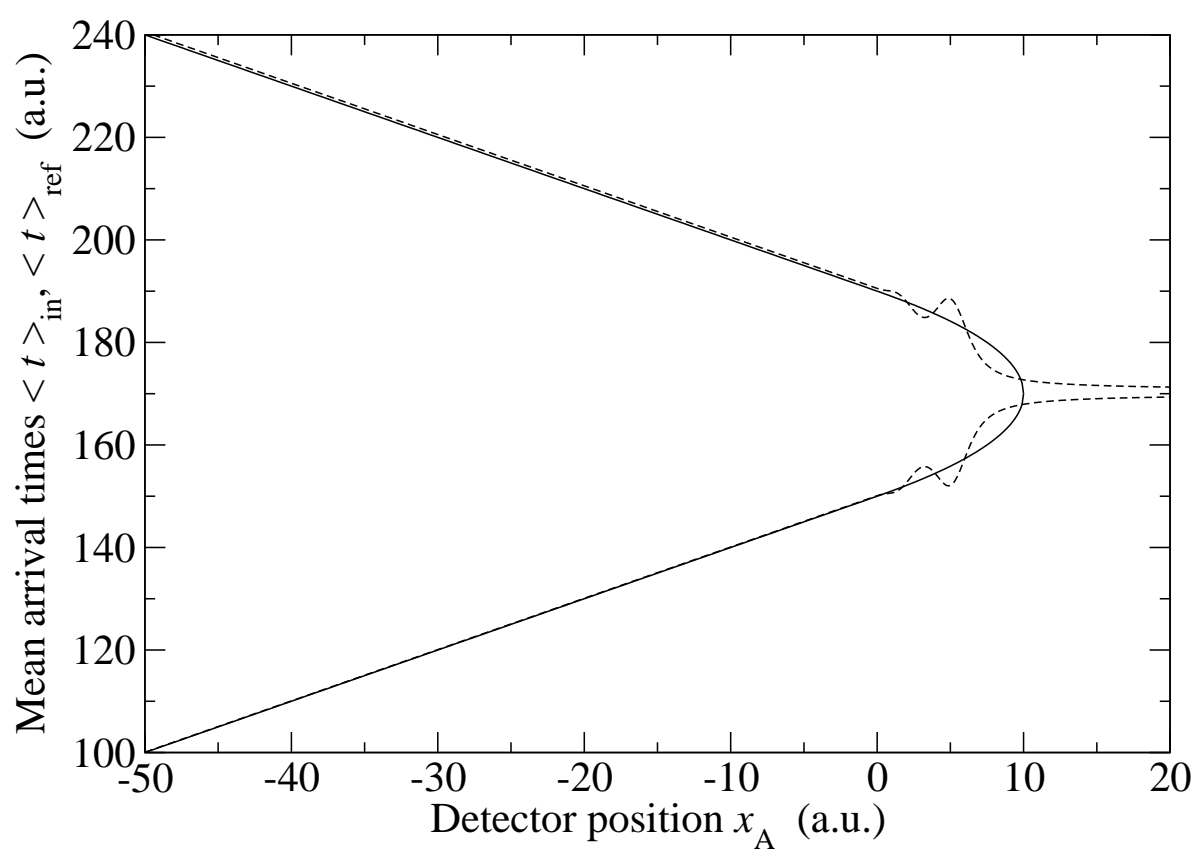

Figure 6.8: Mean quantum arrival times $\langle t\rangle_{\text {in }}$ (dashed line, lower branch) and $\langle t\rangle_{\text {ref }}$ (dashed line, upper branch) in the presence of a linear potential ramp $(F=0.1)$ located in the right half-space. For comparison, their classical counterparts are shown (solid line), where the classical turning point is $x_{\mathrm{turn}}=10$. The state at $t=0$ is a minimum-uncertainty Gaussian wave packet with $\langle x\rangle=-150,\langle v\rangle=1, \Delta x=20$ in atomic units. The small deviation from the classical result in the free region is due to the fact that the quantum average of the inverse velocity does not equal the inverse of the mean velocity.

$v_{0}=2 m V_{0} b^{2} / \hbar^{2}$ and $x=b \xi$, the stationary Schrödinger equation for scattering eigenfunctions $\varphi_{k}(x)$ reads

$$
\left(-\frac{\mathrm{d}^{2}}{\mathrm{~d} x^{2}}-v_{0} \operatorname{sech}^{2}(x)\right) \varphi_{k}(x)=k^{2} \varphi_{k}(x),
$$

where $k^{2}>0$. This equation is analytically solvable for

$$
v_{0}=n(n+1), \quad n=0,1,2, \ldots,
$$

where the solutions may be constructed by suitable raising operators starting from the free solution for $n=0$ [131] or from a direct attack on the differential equation [132]. The result for $n=1$ is given by

$$
\varphi_{k}(x, n=1)=(k+\mathrm{i} \tanh x) \mathrm{e}^{\mathrm{i} k x} .
$$

To obtain asymptotic reflection and transmission coefficients, one has to compare Eq. (6.88) with the usual asymptotic parameterizations for scattering eigenfunctions corresponding to a plane wave coming in from the left,

$$
\begin{aligned}
\lim _{x \rightarrow-\infty} \varphi_{k}(x) & =\mathrm{e}^{\mathrm{i} k x}+R(k) \mathrm{e}^{-\mathrm{i} k x}, \\
\lim _{x \rightarrow \infty} \varphi_{k}(x) & =T(k) \mathrm{e}^{\mathrm{i} k x} .
\end{aligned}
$$


This yields

$$
\begin{aligned}
& R^{(1)}(k) \equiv 0, \\
& T^{(1)}(k)=\frac{k+\mathrm{i}}{k-\mathrm{i}}=\mathrm{e}^{2 \mathrm{i} \arctan (1 / k)},
\end{aligned}
$$

i.e. a vanishing reflection probability for all $k$. The correct solution that corresponds to the asymptotic conditions is obtained from Eq. (6.88) through dividing it by $k-\mathrm{i}$, i.e.

$$
\varphi_{k}(x, 1)=\frac{k+\mathrm{i} \tanh x}{k-\mathrm{i}} \mathrm{e}^{\mathrm{i} k x} .
$$

The solutions for larger $n$ have a similar form [132], where asymptotically holds

$$
\begin{aligned}
& R^{(n)}(k)=0 \\
& T^{(n)}(k)=\frac{(k+\mathrm{i})(k+2 \mathrm{i}) \cdots(k+n \mathrm{i})}{(k-\mathrm{i})(k-2 \mathrm{i}) \cdots(k-n \mathrm{i})}=\exp \left(2 \mathrm{i} \sum_{j=1}^{n} \arctan (j / k)\right) .
\end{aligned}
$$

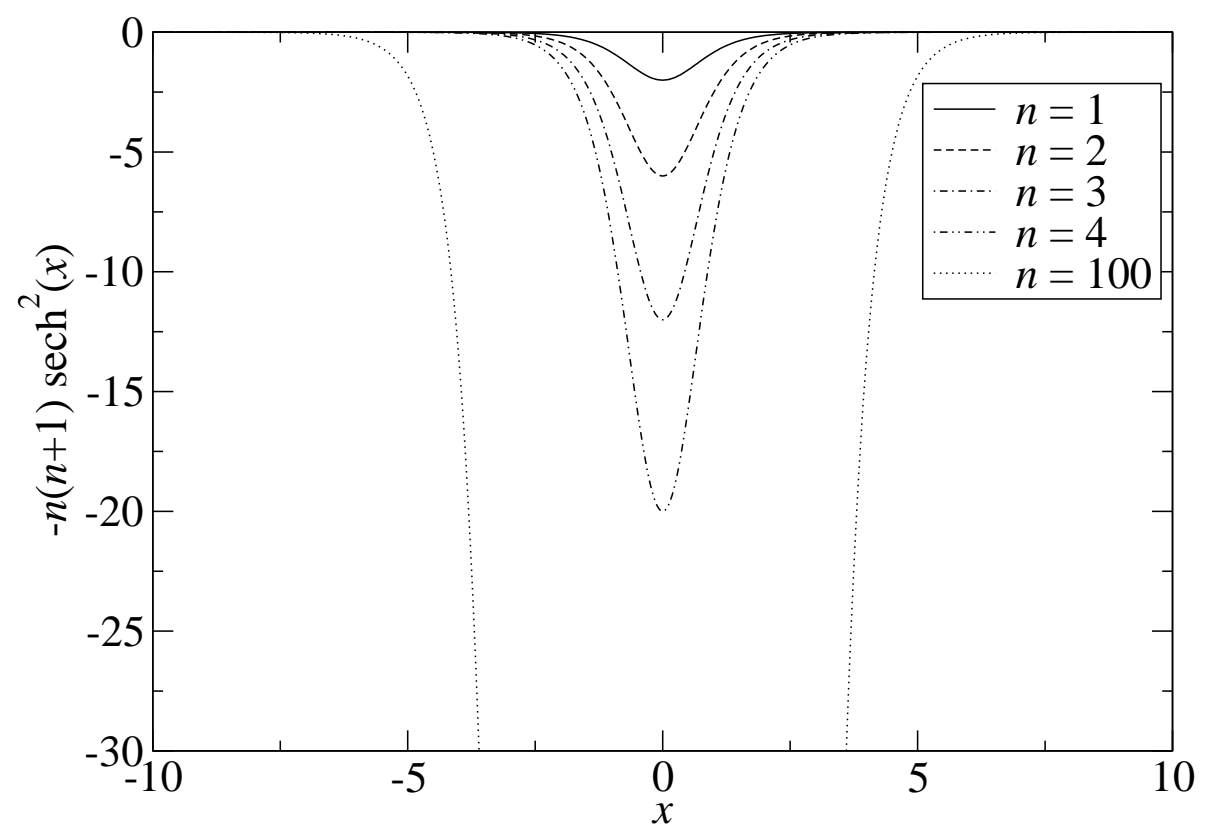

Figure 6.9: Reflectionless potentials $-n(n+1) \operatorname{sech}^{2}(x)$ for some $n$. Even for large $n$ a numerically asymptotic region may be identified (here e.g. $x=10$ ).

Although one is interested in scattering situations, it is interesting to note that the potential $-n(n+1) \operatorname{sech}(x)$ also possesses bound state solutions. They are related to the scattering solutions by the poles of the transmission coefficient $T^{(n)}(k)$ in the upper imaginary plane, i.e. for $k=\mathrm{i}, 2 \mathrm{i}, \ldots, n \mathrm{i}$. Moreover, the difference of the phase shift of $T^{(n)}(k)=\exp \left(\mathrm{i} \delta_{n}(k)\right)$ at $k=0$ and $k \rightarrow \infty$ counts $\pi$ times the number of bound states,

$$
\delta_{n}(0)-\delta_{n}(\infty)=2 \sum_{j=1}^{n} \frac{\pi}{2}=n \pi,
$$


a result that is known as Levinson's theorem [133, 134]. The occurrence of bound states may considerably change the properties of arrival times [125].

In the following, the mean arrival time of a particle crossing a reflectionless potential is investigated by means of the operational approach of this work. From Fig. 6.9 it is seen that even for large values of $n$ an asymptotic region is well defined, where $n(n+1) \operatorname{sech}^{2}(x)$ vanishes, at least numerically. The mean arrival times at an asymptotic point to the right of the potential for a wave packet coming in from the far left are given by Eq. (6.48), where with Eqs. (6.95) and (6.90)

$$
\arg \varphi_{k}(x)=k x+2 \sum_{j=1}^{n} \arctan (j / k) .
$$

Inserting Eq. (6.97) into Eq. (6.48) for the arrival point $x=x_{\mathrm{A}}$ yields

$$
\langle t\rangle_{n}=\frac{m}{\hbar} \int_{0}^{\infty} \mathrm{d} k|\widetilde{\psi}(k)|^{2} \frac{1}{k}\left(\left|x_{0}\right|+x_{\mathrm{A}}-\sum_{j=1}^{n} \frac{2 j}{k^{2}+j^{2}}\right)
$$

The integration over the sum in Eq. (6.98) provides the quantum analogue of the

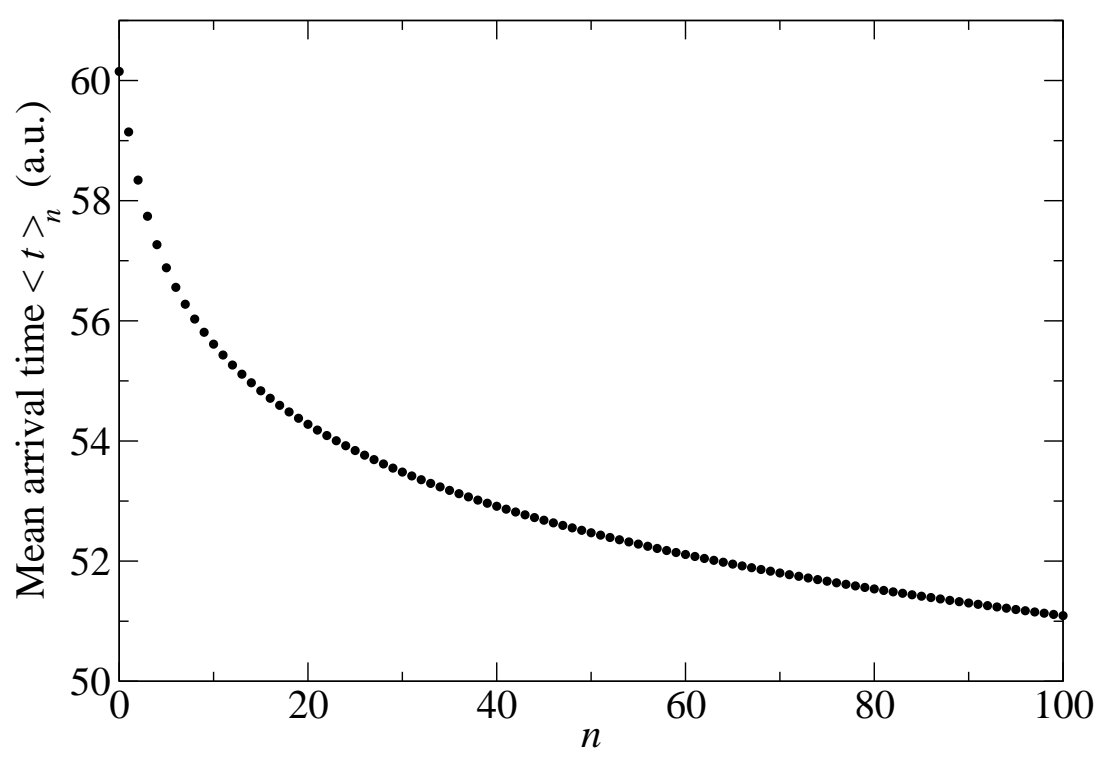

Figure 6.10: Mean arrival time $\langle t\rangle_{n}$ for a particle crossing a reflectionless potential $-n(n+1) \operatorname{sech}^{2}(x), n=0,1,2, \ldots$ The arrival time is measured at $x_{\mathrm{A}}=10$, the state at $t=0$ is a minimum-uncertainty Gaussian wave packet with $\langle x\rangle=-50$, $\langle v\rangle=1, \Delta x=10$ in atomic units.

time advance derived in Eq. (4.7) of Ref. [130]. It should be noted that this time advance can be made arbitrary large, since for large $n$ the sum in Eq. (6.98) tends to infinity,

$$
\sum_{j=1}^{n} \frac{2 j}{k^{2}+j^{2}}=\Psi(n+1-\mathrm{i} k)+\Psi(n+1+\mathrm{i} k)-\Psi(1-\mathrm{i} k)-\Psi(1+\mathrm{i} k),
$$


where $\Psi(x)=\Gamma^{\prime}(x) / \Gamma(x)$ is the Digamma function (related to the Gamma function $\Gamma(x))$ and $\Psi(x) \rightarrow \infty$ for $x \rightarrow \infty$ [129]. This means that the average arrival time decreases for increasing $n$, i.e. when adding more bound states. Clearly, this is due to the attractive nature of the potential which accelerates the atom, an effect that has been recently investigated in detail for square well potentials [125]. In this reference, causality aspects are discussed, too.

A plot of the mean arrival time $\langle t\rangle_{n}$ versus $n$ is shown in Fig. 6.10. Here, for $n=0$ the mean free arrival time is recovered, whereas for larger $n$ the arrival time is smaller than the free time. For large $n$, Eq. (6.99) has an asymptotic expansion given by [129]

$$
\sum_{j=1}^{n} \frac{2 j}{k^{2}+j^{2}} \sim \ln (n), \quad n \rightarrow \infty
$$

and thus the time advance is logarithmic in $n$ which agrees with a classical derivation of the time advance for large potential strength [130]. 


\section{Chapter 7}

\section{Conclusion}

The purpose of the present work is the investigation and development of operational models for quantum arrival times. The particular importance of this issue is due to the lack of satisfactory measurement-based approaches regarding the multitude of proposals for ideal arrival-time distributions. Moreover, the recent progress in manipulating single quantum systems at very low temperatures is a portent of the possibilities to observe some of the time-related phenomena in quantum mechanics in the near future, as for instance the Hartman effect [18] or the simultaneous-arrival effect [135]. For this, it is essential to know what version of the various predicted ideal quantities a particular experiment really measures.

In this work, the quantum optical arrival-time model of Muga et al. [23, 24] has been investigated in detail and has been further developed. It is shown to represent a very powerful approach, yielding a variety of ideal quantities in particular limits. The central idea of the model is to consider a moving two-level atom in one dimension, that is initially prepared in the ground state and impinges on a spatially localized laser beam. When entering the laser field, the atom becomes excited and may emit a photon, where the temporal distribution of the first spontaneously emitted photon is taken as an approximation of the arrival-time distribution of the free atom at the laser field.

As in Ref. [24], the photon-emission probabilities for a moving two-level atom are derived in this work by means of the quantum jump approach [85, 86, 87]. Within this approach, a conditional Hamiltonian is defined which governs the time evolution of the atom before the detection of the first photon, thus of the "undetected" atom. The decreasing norm of the conditionally time-developed state corresponds to the probability of no photon detection until time $t$ and its negative derivative corresponds to the first-photon distribution under consideration. Additionally, the quantum jump approach provides the reset state of the atom right after a detection. In the absence of detuning, the model basically depends on two parameters: the decay rate $\gamma$ of the upper level of the atom and the position-dependent Rabi frequency, $\Omega(x)=\Omega f(x)$, which describes the coupling between the spatially localized laser beam and the atomic dipole moment. For arrivals at $x=x_{\mathrm{A}}$, the laser has to designate this position, e.g. $\Omega(x)=\Omega \Theta\left(x-x_{\mathrm{A}}\right)$.

To relate the operational first-photon distribution to ideal quantum arrival-time 
distributions, limiting cases with respect to these two parameters are considered in Sections 3.2.2, 3.2.3 and 3.2.4. It turns out that the disturbing effects of reflection and delay appear in the strong and weak laser regime, respectively. These problems can be solved by a proper combination of parameter limits and mathematical procedures. On the one hand, the detection delay caused by the internal time scale of the atom can be eliminated by a deconvolution with the waiting time distribution for an atom at rest and, together with the limit $\gamma \rightarrow \infty$ (or $\Omega \rightarrow 0$ ), one obtains the quantum mechanical flux, $J\left(t, x_{\mathrm{A}}\right)$ [24]. This is an important result, since the flux is the classical version of the arrival-time distribution. Although its quantum counterpart is not necessarily positive not even for wave packets with only positive momenta, and thus cannot be considered as a true probability density, the scheme described above shows the measurability of the quantum mechanical probability flux, in contrast to the suggestions made in some textbooks [97].

On the other hand, the non-detection of atoms due to reflections at a strong laser field may be compensated by a normalization of the distribution. In combination with the limit $\gamma \rightarrow \infty, \gamma / \Omega=$ const., which minimizes the emission delay, one obtains an ideal distribution related to a positive version of the local kinetic energy density as shown in Section 3.5. This provides for the first time an operational understanding of this quantity [113].

Moreover, it has been shown in Section 3.3 that instead of examining a laser field that is assumed to be extended over the right half-space, $x \geq 0$, it is possible to consider a very narrow laser beam modeled by $\Omega(x) \sim \Omega \delta\left(x-x_{\mathrm{A}}\right)$. This modification yields another interesting case when deriving the normalized first-photon distribution in the limit $\Omega \rightarrow 0, \gamma \rightarrow \infty$, namely the density of the state times the average velocity, $v_{0}\left|\psi\left(x_{\mathrm{A}}, t\right)\right|^{2}$. It can be seen as a semiclassical time-of-arrival distribution.

It is a particularly interesting fact that previous arrival-time models which are based on the absorption rate in imaginary potentials [6] turn out to be included in the quantum optical approach of this work. Indeed, Section 3.4 shows that in the limit $\gamma \rightarrow \infty, \gamma / \Omega^{2}=$ const. and for a resonant laser the atom is mainly in the ground state and its conditional time evolution is governed by a one-channel Hamiltonian with an absorbing potential of the form $V=-\mathrm{i} \hbar \Omega^{2} / 2 \gamma$. For detuned lasers, this potential exhibits also a real part. A similar result has been found in Ref. [103] by means of the Feshbach projection technique. The one-channel limit of the laser model has an important consequence: it can be applied to the reset state of the atom right after a photon emission, which yields a "reset state" after an absorption in the one-channel picture. Remarkably, the resulting state turns out to be identical with the reset state put forward by Blanchard and Jadczyk within their event-enhanced quantum theory [80]. Thus, it has been shown that the laser model guarantees a physical explanation for the use of those reset states after an absorption.

Beside the quantum mechanical flux, $J(t, x)$, and the semiclassical expression $v_{0}|\psi(x, t)|^{2}$, Kijowski's arrival-time distribution, $\Pi_{\mathrm{K}}(t, x)$, is the most accepted expression for free particles from a fundamental point of view (see Sections 2.1.4 and 2.1.5). Two of its important properties are the bilinearity with respect to the wave function and its positivity. These are features not compatible with the previous 
procedures, since the deconvolution destroys the positivity and the ordinary normalization destroys the bilinearity. Therefore, a normalization on the level of the corresponding operators, recently proposed by Brunetti and Fredenhagen [27], has been applied to the first-photon approach in Chapter 5. As a remarkable result, it has been shown in this work that the operator-normalized first-photon distribution in the limit of vanishing delay, $\gamma \rightarrow \infty, \gamma / \Omega=$ const., tends to $\Pi_{K}\left(t, x_{\mathrm{A}}\right)$ [84]. This is the first relation between an operational quantity and Kijowski's distribution. A more realistic version of this connection has been found for a fixed decay rate $\gamma$, taking only $\Omega \rightarrow \infty$, in which case a deconvolution with respect to the delay caused by $\gamma$ again yields Kijowski's distribution. Clearly, the operator-normalization procedure can be applied to the one-channel model with an absorbing potential as well, in which case the operator-normalized absorption rate in the strong detection limit $V \rightarrow \infty$ leads to $\Pi_{\mathrm{K}}\left(t, x_{\mathrm{A}}\right)$.

The various relations between the operational quantities investigated in this work and ideal quantities are summarized schematically in Fig. 7.1. It has to be emphasized that the quantum optical arrival-time model is a powerful approach, which yields not only different versions of ideal arrival-time distributions, but also a positive version of local kinetic energy densities.

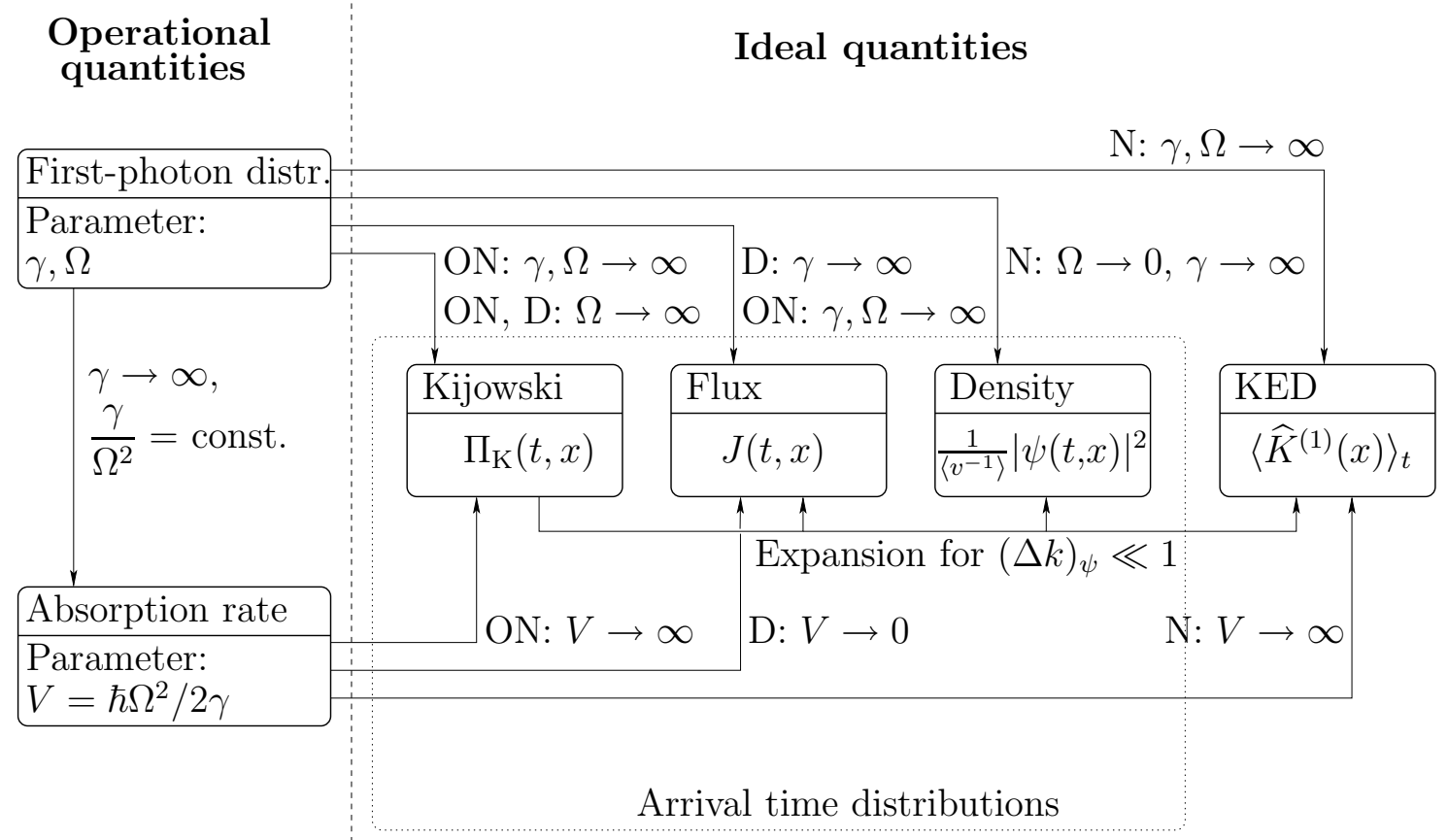

Figure 7.1: Schematic picture of the relations between operational and ideal quantities investigated in this work. Abbreviations are: ON: operator normalization, N: normalization, D: deconvolution, KED: kinetic energy density.

As an interesting aspect, it has been found in Section 3.5.4 that on a fundamental level the ideal quantities depicted in Fig. 7.1 are closely connected in terms of an expansion of Kijowski's distribution for wave packets which are peaked around some 
$p_{0}$ in momentum space. In particular, the difference $\left\langle\widehat{K}^{(1)}(x)-\widehat{K}^{(2)}(x)\right\rangle_{t}$ of two types of local kinetic energy densities, emphasized in chemical physics as an important quantity to define molecular fragments [105], turns out to be proportional to the difference of Kijowski's distribution and the flux. Further research in this direction is needed to clarify this fundamental relationship.

In contrast to the axiomatic approaches of Kijowski [10] and Giannitrapani [11], the operational first-photon model is easily applicable to particles interacting with some external potential. The interaction case is studied in Chapter 6, where the onechannel limit of the laser model has been used for convenience. By normalizing the absorption rate in a narrow spatial interval around $x=x_{\mathrm{A}}$ on the level of operators, a generalization of Kijowski's arrival-time distribution is found which depends solely on the phase of the wave function at $x_{\mathrm{A}}$ in the absence of the measurement potential [122]. In particular, the average arrival time derived with this distribution turns out to be the average of the Wigner phase time [19] over the initial state. This generalization is an important result of this work. It allows for the first time the investigation of arrival-time distributions in the presence of interactions, and in particular of tunneling times, within an operational framework. Several examples are given in Sections 6.4, 6.5 and 6.6 for rectangular barriers, linear potentials, and transparent potentials. The Hartman effect [18], i.e. the independence of the tunneling time with respect to the barrier width, is recovered and it is shown to hold for arbitrary wide barriers, without any transition to the classical-like, ultra-opaque regime investigated in Ref. [64].

Since the concept of operator normalization was introduced from a mathematical point of view to compensate for reflection and transmission losses, it remained to be investigated how such an intervention could be performed in practice. In Section 5.4 it is shown that the operator-normalized first-photon distribution can be seen as the usual first-photon distribution for a pre-filtered wave packet. This filtering procedure has been studied in detail and properly designed filter potentials are derived by means of inverse scattering methods. Thus, the combination of filter potential and first-photon measurement yields a "recipe" how to measure Kijowski's distribution, similar to the combination of first-photon measurements and a deconvolution for the flux.

Beside arrival times, quantum dwell times have also been investigated in this work. It has been shown in Section 4.2 that the first and second moment of an ideal dwell-time distribution that is based on the Ekstein-Siegert dwell-time operator [26] agrees with the corresponding moments of a properly defined flux-flux correlation function. This result is new, at least for the second moment which reflects the quantum features of the distribution. It may help to relate the ideal distribution of dwell times to an operational one, as has been done successfully for arrival-time distributions. A first proposal towards an operational understanding of the flux-flux correlation function in terms of photon-photon correlations is given in Section 4.3, but so far no direct correspondence to dwell-time distribution has been obtained. Thus, a successful operational approach to quantum dwell-time distributions remains as a challenge for future research. 


\section{Appendix A}

\section{Kijowski's axiomatic approach to arrival-time distributions}

In this appendix, the axiomatic approach of Kijowski [10] to quantum arrival-time distributions of free particles with positive momenta is reviewed. Starting from the classical case, the axioms leading to Eq. (2.14) are presented. Kijowski's threedimensional formulation is translated to the one-dimensional case according to the need of the present work.

\section{A.1 The classical case}

In classical mechanics, an ensemble of free particles with only positive momentum components is described by the phase-space distribution function $\varrho(x-p t / m, p)$ with $\varrho(x, p<0)=0$. For convenience, $\varrho(x, p)$ is assumed to be normalized, $\int \mathrm{d} x \int \mathrm{d} p \varrho(x, p)=1$. As a start, the arrival point is chosen to be $x_{\mathrm{A}}=0$.

Consider linear functionals of $\varrho$, denoted by $\Pi[\varrho]$, which satisfy the following conditions:

(i) $\Pi[\varrho] \geq 0$ for $\varrho \geq 0$ (positivity),

(ii) $\int_{-\infty}^{\infty} \mathrm{d} t \Pi[\varrho]=1$ (normalization).

(iii) The distribution function $\varrho^{(\mathrm{TR})}(x, p)=\varrho(-x, p)$ arises from $\varrho(x, p)$ through reflection at $x=0(x \rightarrow-x, p \rightarrow-p)$ and time reversal $(x \rightarrow x, p \rightarrow-p)$. Then the third condition reads:

$$
\Pi\left[\varrho^{(\mathrm{TR})}\right]=\Pi[\varrho] .
$$

According to Ref. [10], this distinguishes the particular arrival point $x_{\mathrm{A}}=0$ from others.

The fourth axiom of Kijowski is related to the Galilei invariance with respect to the plane of arrival and can be omitted in the one-dimensional case. 
It can be easily shown that one example for a linear functional satisfying (i)-(iii) is the classical flux at $x_{\mathrm{A}}=0$, given by Eq. (2.2):

$$
\Pi^{\mathrm{cl}}[\varrho]=J^{\mathrm{cl}}(t, 0) .
$$

In Ref. [10], the following theorem is proved:

Theorem 1 (Kijowski): Let $\Pi$ be a linear functional of $\varrho$ satisfying the above conditions (i)-(iii) and in addition

$$
\int_{-\infty}^{\infty} \mathrm{d} t \Pi[\varrho] t^{2}<\infty
$$

If one defines the mean values $\bar{t}_{\Pi}=\int_{-\infty}^{\infty} \mathrm{d} t \Pi[\varrho] t$ and $\bar{t}_{\mathrm{cl}}=\int_{-\infty}^{\infty} \mathrm{d} t \Pi^{\mathrm{cl}}[\varrho] t$ then one has for all of the above $\Pi$ :

(1) $\bar{t}_{\Pi}=\bar{t}_{\mathrm{cl}}$,

(2) $\int_{-\infty}^{\infty} \mathrm{d} t\left(t-\bar{t}_{\Pi}\right)^{2} \Pi[\varrho] \geq \int_{-\infty}^{\infty} \mathrm{d} t\left(t-\bar{t}_{\mathrm{cl}}\right)^{2} \Pi^{\mathrm{cl}}[\varrho]$

Moreover, if equality holds in (2), then $\Pi=\Pi^{\mathrm{cl}}$.

In other words, among the distributions satisfying (i)-(iii), the classical flux $J^{\mathrm{cl}}(t, 0)$ is the one with minimal variance. This can be seen as a kind of correspondence to an ideal detector which measures arrival times as precisely as possible.

\section{A.2 The quantum case}

To derive a quantum mechanical arrival-time distribution, Kijowski translated the classical axioms into the quantum language. This is reviewed in the following, where the notation of Ref. [10] is slightly modified.

Let $\widehat{\Pi}$ be an (in general unbounded) operator on the space $\mathcal{D}$ of normalized wave functions with only positive momentum components, $\mathcal{D}=\left\{\psi \in L^{2}(\mathbb{R}), \widetilde{\psi}(k)=\right.$ 0 for $\left.k<0,\left\|\psi_{t}\right\|^{2}=1\right\}$. The bilinear form $\Pi\left[\psi_{t}\right]=\left\langle\psi_{t}|\widehat{\Pi}| \psi_{t}\right\rangle$ will have the meaning of a quantum arrival-time distribution. $\psi_{t}$ shall belong to the domain of $\widehat{\Pi}$. In analogy to the classical case Kijowski postulates:

(i) $\widehat{\Pi} \geq 0$, i.e. $\left\langle\psi_{t}|\widehat{\Pi}| \psi_{t}\right\rangle \geq 0$,

(ii) $\int_{-\infty}^{\infty} \mathrm{d} t\left\langle\psi_{t}|\widehat{\Pi}| \psi_{t}\right\rangle=1$

(iii) Let $\widehat{\pi}$ the (unitary) parity operator and $\widehat{\tau}$ the (anti-unitary) time reversal operator. As in the classical case, the combined action of both operators 
yields $\widehat{x} \rightarrow-\widehat{x}, \widehat{p} \rightarrow \widehat{p}$. One has $\left\langle k|\widehat{\tau} \widehat{\pi}| \psi_{t}\right\rangle=\overline{\widetilde{\psi}_{t}(k)}$. Then condition (iii) of the classical case reads in the quantum case

$$
\left\langle\psi_{0}|\widehat{\Pi}| \psi_{0}\right\rangle=\left\langle\widehat{\tau} \widehat{\pi} \psi_{0}|\widehat{\Pi}| \widehat{\tau} \widehat{\pi} \psi_{0}\right\rangle
$$

or, equivalently,

$$
\Pi\left[\widetilde{\psi}_{0}\right]=\Pi\left[\widetilde{\widetilde{\psi}_{0}}\right]
$$

A particular operator which satisfies (i)-(iii) is a positive quantization of the classical phase-space function $\mathcal{J}(x, p ; 0)$ of Eq. (2.3), namely

$$
\widehat{\Pi}_{\mathrm{K}}=\frac{1}{m} \widehat{p}^{1 / 2} \delta(\widehat{x}) \widehat{p}^{1 / 2}
$$

Note that the quantum mechanical flux (2.11) does not satisfy condition (i). The following theorem is the quantum analogue to Theorem 1 [10]:

Theorem 2 (Kijowski): $\quad$ Let $\widehat{\Pi}$ be an operator satisfying (i)-(iii) and in addition

$$
\int_{-\infty}^{\infty} \mathrm{d} t\left\langle\psi_{t}|\widehat{\Pi}| \psi_{t}\right\rangle t^{2}<\infty
$$

If one defines the mean values $\bar{t}_{\widehat{\Pi}}=\int_{-\infty}^{\infty} \mathrm{d} t\left\langle\psi_{t}|\widehat{\Pi}| \psi_{t}\right\rangle t$ and $\bar{t}_{\mathrm{K}}=\int_{-\infty}^{\infty} \mathrm{d} t\left\langle\psi_{t}\left|\widehat{\Pi}_{\mathrm{K}}\right| \psi_{t}\right\rangle t$ then:

(1) $\bar{t}_{\widehat{\Pi}}=\bar{t}_{\mathrm{K}}$

(2) $\int_{-\infty}^{\infty} \mathrm{d} t\left(t-\bar{t}_{\widehat{\Pi}}\right)^{2}\left\langle\psi_{t}|\widehat{\Pi}| \psi_{t}\right\rangle \geq \int_{-\infty}^{\infty} \mathrm{d} t\left(t-\bar{t}_{\mathrm{K}}\right)^{2}\left\langle\psi_{t}\left|\widehat{\Pi}_{\mathrm{K}}\right| \psi_{t}\right\rangle$

Moreover, if equality holds in (2) for all $\psi$, then $\left\langle\psi_{t}|\widehat{\Pi}| \psi_{t}\right\rangle=\left\langle\psi_{t}\left|\widehat{\Pi}_{K}\right| \psi_{t}\right\rangle$.

The distribution function with minimal variance among all possible distributions satisfying (i)-(iii) will be called "Kijowski's distribution" in the present work. It reads

$$
\Pi_{\mathrm{K}}\left(t, x_{\mathrm{A}}=0\right)=\left\langle\psi_{t}\left|\widehat{\Pi}_{\mathrm{K}}\right| \psi_{t}\right\rangle=\frac{\hbar}{2 \pi m}\left|\int_{0}^{\infty} \mathrm{d} k \widetilde{\psi}(k) \sqrt{k} \mathrm{e}^{-\mathrm{i} \hbar k^{2} t / 2 m}\right|^{2} .
$$

For an arbitrary arrival point $x=x_{\mathrm{A}}$, the delta function in Eq. (A.5) has to be replaced by $\delta\left(\widehat{x}-x_{\mathrm{A}}\right)$ and this leads to an additional phase factor in the distribution,

$$
\Pi_{\mathrm{K}}\left(t, x_{\mathrm{A}}\right)=\left\langle\psi_{t}\left|\widehat{\Pi}_{\mathrm{K}}\right| \psi_{t}\right\rangle=\frac{\hbar}{2 \pi m}\left|\int_{0}^{\infty} \mathrm{d} k \widetilde{\psi}(k) \sqrt{k} \mathrm{e}^{-\mathrm{i} \hbar k^{2} t / 2 m} \mathrm{e}^{\mathrm{i} k x_{\mathrm{A}}}\right|^{2} .
$$




\section{Appendix B}

\section{Transfer matrix method}

In the present work, the scattering eigenfunctions for particular one-dimensional scattering problems have to be derived. It is shown in the following, that a systematical approach for piecewise-constant potentials can be given by means of transfer matrices. This is well known for one-channel scattering problems [136], and an extension for two-channel Hamiltonians of the form (3.55) is provided in Section B.2. Moreover, it is shown that in the limit of decomposing an arbitrary continuous potential by infinitely many barriers with vanishing width, a differential equation for the position-dependent scattering amplitudes can be derived for the one-channel case. That can be used for a numerical solution of the one-channel Schrödinger equation with smooth potentials. A similar approach has been given in Refs. [137, 138].

\section{B.1 One-channel case}

To calculate the transmission and reflection coefficients of one-dimensional scattering problems with piecewise-constant potentials, the transfer matrix method of Ref. [136] is used and "matching" matrices

$$
\mathbf{M}_{i}(x)=\left[\begin{array}{cc}
\mathrm{e}^{\mathrm{i} k_{i} x} & \mathrm{e}^{-\mathrm{i} k_{i} x} \\
k_{i} \mathrm{e}^{\mathrm{i} k_{i} x} & -k_{i} \mathrm{e}^{-\mathrm{i} k_{i} x}
\end{array}\right]
$$

are defined for each section $i$ of the constant potential $V_{i}$, where

$$
k_{i}=\sqrt{k^{2}-2 m V_{i} / \hbar^{2}}
$$

with $\operatorname{Im} k_{i} \geq 0$. The bracket notation in Eq. (B.1) has been chosen to avoid confusion with the $2 \times 2$-matrices for internal states of the two-level atom defined by $\left(\begin{array}{l}1 \\ 0\end{array}\right) \equiv|1\rangle$ and $\left(\begin{array}{l}0 \\ 1\end{array}\right) \equiv|2\rangle$. The general solution of the stationary Schrödinger equation to positive energy $E_{k}=\hbar^{2} k^{2} / 2 m$ in section $i$ is given by

$$
\phi_{k}^{[i]}(x)=\frac{1}{\sqrt{2 \pi}}\left(A_{i} \mathrm{e}^{\mathrm{i} k_{i} x}+B_{i} \mathrm{e}^{-\mathrm{i} k_{i} x}\right) .
$$

The index 0 is always used for the leftmost section, and the rest is numbered rightwards and consecutively. The boundary point between sections $i$ and $i+1$ is denoted 
by $x_{i+1}$, so that the matching conditions take the general form

$$
\mathbf{M}_{i}\left(x_{i+1}\right)\left[\begin{array}{l}
A_{i} \\
B_{i}
\end{array}\right]=\mathbf{M}_{i+1}\left(x_{i+1}\right)\left[\begin{array}{l}
A_{i+1} \\
B_{i+1}
\end{array}\right] .
$$

Multiplying both sides by $\mathbf{M}_{i}^{-1}\left(x_{i+1}\right)$ yields

$$
\left[\begin{array}{l}
A_{i} \\
B_{i}
\end{array}\right]=\mathbf{T}(i, i+1)\left[\begin{array}{l}
A_{i+1} \\
B_{i+1}
\end{array}\right]
$$

where the transfer matrix connecting the amplitudes of regions $i$ and $i+1$ is given by

$$
\mathbf{T}(i, i+1)=\mathbf{M}_{i}^{-1}\left(x_{i+1}\right) \mathbf{M}_{i+1}\left(x_{i+1}\right) .
$$

One may similarly obtain transfer matrices for non-contiguous regions $i$ and $j \geq i+2$ by multiplying all the intermediate one-step transfer matrices,

$$
\mathbf{T}(i, j)=\mathbf{T}(i, i+1) \mathbf{T}(i+1, i+2) \ldots . \mathbf{T}(j-1, j),
$$

and this yields

$$
\left[\begin{array}{l}
A_{i} \\
B_{i}
\end{array}\right]=\mathbf{T}(i, j)\left[\begin{array}{l}
A_{j} \\
B_{j}
\end{array}\right]
$$

In the following subsections the examples treated in the present work are discussed. It will be shown in Section B.1.4 that the transfer matrix method may be adapted to continuous potentials leading in that case to differential equations for the amplitudes.

\section{B.1.1 Potential barrier}

In the case of a potential barrier $U(x)=U \chi_{[a, b]}(x)$, where $\chi_{[a, b]}$ is the characteristic function of the interval $x_{1}=a \leq x \leq x_{2}=b$, there are three sections with wave numbers $k_{0}=k_{2} \equiv k$ and $k_{1}=\left[k^{2}-2 m U / \hbar^{2}\right]^{1 / 2} \equiv \kappa, \operatorname{Im} \kappa>0$. Defining

$$
l=b-a, \quad s=a+b,
$$

it follows with Eq. (B.6) that

$$
\mathbf{T}(1,2)=\frac{1}{2}\left[\begin{array}{l}
\left(1+\frac{k}{\kappa}\right) \mathrm{e}^{\mathrm{i}(k-\kappa) b}\left(1-\frac{k}{\kappa}\right) \mathrm{e}^{-\mathrm{i}(k+\kappa) b} \\
\left(1-\frac{k}{\kappa}\right) \mathrm{e}^{\mathrm{i}(k+\kappa) b}\left(1+\frac{k}{\kappa}\right) \mathrm{e}^{-\mathrm{i}(k-\kappa) b}
\end{array}\right]
$$

and with Eq. (B.7) that

$$
\mathbf{T}(0,2)=\left[\begin{array}{cc}
{\left[\cos (\kappa l)-\frac{\mathrm{i}}{2}\left(\frac{\kappa}{k}+\frac{k}{\kappa}\right) \sin (\kappa l)\right] \mathrm{e}^{\mathrm{i} k l}} & -\frac{\mathrm{i}}{2}\left(\frac{\kappa}{k}-\frac{k}{\kappa}\right) \mathrm{e}^{-\mathrm{i} k s} \sin (\kappa l) \\
\frac{\mathrm{i}}{2}\left(\frac{\kappa}{k}-\frac{k}{\kappa}\right) \mathrm{e}^{\mathrm{i} k s} \sin (\kappa l) & \left.\left[\cos (\kappa l)+\frac{\mathrm{i}}{2}\left(\frac{\kappa}{k}+\frac{k}{\kappa}\right) \sin (\kappa l)\right] \mathrm{e}^{-\mathrm{i} k l}\right]
\end{array}\right] .
$$


For waves incident from the left $(x<0)$ the boundary conditions are $A_{0}=1$ and $B_{2}=0$ and one can solve Eq. (B.8) for the other amplitudes, which yields

$$
\begin{aligned}
& A_{2}=\left[T_{11}(0,2)\right]^{-1}=\mathrm{e}^{-\mathrm{i} k l} / D, \\
& B_{0}=T_{21}(0,2) A_{2}^{+}=\frac{\mathrm{i}}{2}\left(\frac{\kappa}{k}-\frac{k}{\kappa}\right) \sin (\kappa l) \mathrm{e}^{2 \mathrm{i} k a} / D, \\
& A_{1}=T_{11}(1,2) A_{2}^{+}=\frac{1}{2}\left(1+\frac{k}{\kappa}\right) \mathrm{e}^{\mathrm{i} k a} \mathrm{e}^{-\mathrm{i} \kappa b} / D, \\
& B_{1}=T_{21}(1,2) A_{2}^{+}=\frac{1}{2}\left(1-\frac{k}{\kappa}\right) \mathrm{e}^{\mathrm{i} k a} \mathrm{e}^{\mathrm{i} \kappa b} / D,
\end{aligned}
$$

with the common denominator

$$
D=\cos (\kappa l)-\frac{\mathrm{i}}{2}\left(\frac{\kappa}{k}+\frac{k}{\kappa}\right) \sin (\kappa l) .
$$

\section{B.1.2 Dirac delta potential}

The scattering amplitudes for the Dirac delta potential $U(x)=u_{0} \delta(x-a)$ can be easily derived from the results of the previous section by setting $U=u_{0} / \epsilon, b=a+\epsilon$ and taking the limit $\epsilon \rightarrow 0$. For the transmission and reflection amplitudes this yields

$$
\begin{aligned}
& A_{2}=\frac{\hbar^{2} k}{\hbar^{2} k+m u_{0}}, \\
& B_{0}=\frac{-m u_{0}}{\hbar^{2} k+m u_{0}} \mathrm{e}^{2 \mathrm{i} k a},
\end{aligned}
$$

whereas $A_{1}$ and $B_{1}$ are irrelevant since the corresponding region vanishes. Eqs. (B.14) and (B.15) can also be obtained by considering the matching conditions for a delta potential at $x=a$.

\section{B.1.3 Potential step}

For a potential step $U>0$ at $x_{1}=a$ and a left incoming plane wave one has two sections with $k_{0} \equiv k, k_{1}=\left[k^{2}-2 m U / \hbar\right]^{1 / 2} \equiv \kappa$. The transfer matrix connecting these two sections is

$$
\mathbf{T}(0,1)=\frac{1}{2}\left[\begin{array}{l}
\left(1+\frac{\kappa}{k}\right) \mathrm{e}^{\mathrm{i}(\kappa-k) a}\left(1-\frac{\kappa}{k}\right) \mathrm{e}^{-\mathrm{i}(\kappa+k) a} \\
\left(1-\frac{\kappa}{k}\right) \mathrm{e}^{\mathrm{i}(\kappa+k) a}\left(1+\frac{\kappa}{k}\right) \mathrm{e}^{-\mathrm{i}(\kappa-k) a}
\end{array}\right] .
$$

With boundary conditions $A_{0}=1$ and $B_{1}=0$ the transmission and reflection amplitudes are given by

$$
\begin{aligned}
& A_{1}=\left[T_{11}(0,1)\right]^{-1}=\frac{2 k}{k+\kappa} \mathrm{e}^{-\mathrm{i}(\kappa-k) a}, \\
& B_{0}=T_{21}(0,1) A_{1}=\frac{k-\kappa}{k+\kappa} \mathrm{e}^{2 \mathrm{i} k a} .
\end{aligned}
$$


Appendix B. Transfer matrix method

\section{B.1.4 Continuous potentials}

A continuous potential $V(x)$ can be seen as the limit of an infinite number of infinitely narrow barriers. This allows to derive the asymptotic reflection and transmission amplitudes by means of the formalism of the previous section.

In the case of infinitely narrow barriers the wave number of Eq. (B.2) becomes position-dependent,

$$
k_{i} \rightarrow \kappa(x)=\sqrt{k^{2}-2 m V(x) / \hbar^{2}},
$$

and so do the amplitudes, $A_{i}, B_{i} \rightarrow A(x), B(x)$. With directly and indirectly position-dependent matching matrices

$$
\mathbf{M}_{i}(x) \rightarrow \mathbf{M}_{\kappa(x)}(x)=\left[\begin{array}{cc}
\mathrm{e}^{\mathrm{i} \kappa(x) x} & \mathrm{e}^{-\mathrm{i} \kappa(x) x} \\
\kappa(x) \mathrm{e}^{\mathrm{i} \kappa(x) x} & -\kappa(x) \mathrm{e}^{-\mathrm{i} \kappa(x) x}
\end{array}\right],
$$

the matching condition at $x$ takes the form

$$
\mathbf{M}_{\kappa(x)}(x)\left[\begin{array}{l}
A(x) \\
B(x)
\end{array}\right]=\mathbf{M}_{\kappa(x+\mathrm{d} x)}(x)\left[\begin{array}{l}
A(x+\mathrm{d} x) \\
B(x+\mathrm{d} x)
\end{array}\right] .
$$

Multiplying both sides by $\mathbf{M}_{\kappa(x+\mathrm{d} x)}^{-1}(x)$ and using $\kappa(x+\mathrm{d} x)-\kappa(x)=\kappa^{\prime}(x) \mathrm{d} x \ll 1$, valid for smooth potentials, one obtains

$$
\left[\begin{array}{l}
A(x+\mathrm{d} x) \\
B(x+\mathrm{d} x)
\end{array}\right]=\left[\begin{array}{cc}
1-\mathrm{i} \kappa^{\prime}(x) x \mathrm{~d} x & \frac{1}{2 \kappa(x)} \kappa^{\prime}(x) \mathrm{e}^{-2 \mathrm{i} \kappa(x) x} \mathrm{~d} x \\
\frac{1}{2 \kappa(x)} \kappa^{\prime}(x) \mathrm{e}^{2 \mathrm{i} \kappa(x) x} \mathrm{~d} x & 1+\mathrm{i} \kappa^{\prime}(x) x \mathrm{~d} x
\end{array}\right]\left[\begin{array}{l}
A(x) \\
B(x)
\end{array}\right]
$$

Subtracting $\left[\begin{array}{l}A(x) \\ B(x)\end{array}\right]$ and comparing the terms of order $\mathrm{d} x$ finally leads to a system of coupled differential equations for the amplitudes,

$$
\frac{\mathrm{d}}{\mathrm{d} x}\left[\begin{array}{l}
A(x) \\
B(x)
\end{array}\right]=\kappa^{\prime}(x)\left[\begin{array}{cc}
-\mathrm{i} x & \frac{\mathrm{e}^{-2 \mathrm{i} \kappa(x) x}}{2 \kappa(x)} \\
\frac{\mathrm{e}^{+2 \mathrm{i} \kappa(x) x}}{2 \kappa(x)} & \mathrm{i} x
\end{array}\right]\left[\begin{array}{c}
A(x) \\
B(x)
\end{array}\right] .
$$

When $A(x)$ and $B(x)$ are determined, they provide a general solution of the Schrödinger equation for smooth potentials $V(x)$ which reads

$$
\phi_{k}(x)=\frac{1}{\sqrt{2 \pi}}\left(A(x) \mathrm{e}^{\mathrm{i} \kappa(x) x}+B(x) \mathrm{e}^{-\mathrm{i} \kappa(x) x}\right) .
$$

The initial condition for a wave coming in from the left is given by $A(-\infty)=1$ and $B(\infty)=0$ and the asymptotic transmission and reflection amplitudes are $A(\infty)$ and $B(-\infty)$. The difficulty with the mixed initial condition can be circumvented in the following way: The asymptotic solution for $x \rightarrow \infty$ is divided formally by $A(\infty)$ and by this one obtains a modified initial condition $\left[\begin{array}{l}1 \\ 0\end{array}\right]$ for $x \rightarrow \infty$. Using this, one can integrate back towards $x \rightarrow-\infty$ to get the solution $\tilde{A}(x)$ and $\tilde{B}(x)$. The desired transmission and reflection amplitudes of the original problem are then given by $A(x)=\tilde{A}(x) / \tilde{A}(-\infty)$ and $B(x)=\tilde{B}(x) / \tilde{A}(-\infty)$. 


\section{B.2 Two-channel case}

For the two-channel case, it is sufficient for this work to restrict the analysis to Hamiltonians of the form (3.55) with piecewise-constant Rabi frequencies $\Omega_{i}$. With $\Omega(x)=\Omega_{i} \chi_{\left[x_{i}, x_{i+1}\right]}(x)$, the conditional Hamiltonian for section $i$ reads

$$
H_{\mathrm{c}}^{(i)}=\frac{\widehat{p}^{2}}{2 m}+\frac{\hbar}{2}\left(\begin{array}{cc}
0 & \Omega_{i} \chi_{\left[x_{i}, x_{i+1}\right]}(\widehat{x}) \\
\Omega_{i} \chi_{\left[x_{i}, x_{i+1}\right]}(\widehat{x}) & -(\mathrm{i} \gamma+2 \delta)
\end{array}\right) .
$$

The eigenvalues and (unnormalized) eigenvectors of the matrix $\frac{1}{2}\left(\begin{array}{cc}0 & \Omega_{i} \\ \Omega_{i} & -(\mathrm{i} \gamma+2 \delta)\end{array}\right)$ are given by

$$
\begin{aligned}
\lambda_{i}^{ \pm} & =-\frac{\mathrm{i} \gamma+2 \delta}{4} \pm \frac{\mathrm{i}}{4} \sqrt{(\gamma-2 \mathrm{i} \delta)^{2}-4 \Omega_{i}^{2}} \\
\left|\lambda_{i}^{ \pm}\right\rangle & =\left(\begin{array}{c}
1 \\
2 \lambda_{i}^{ \pm} / \Omega_{i}
\end{array}\right) .
\end{aligned}
$$

Since $H_{\mathrm{c}}^{(i)}$ is a non-Hermitian operator, the eigenvectors $\left|\lambda_{i}^{ \pm}\right\rangle$are not orthogonal for $\gamma \neq 0$. The general solution of the stationary Schrödinger equation to positive energy $E_{k}=\hbar^{2} k^{2} / 2 m$ in section $i$ is given by

$$
\boldsymbol{\Phi}_{k}^{[i]}(x)=\frac{1}{\sqrt{2 \pi}}\left(A_{i}^{+}\left|\lambda_{i}^{+}\right\rangle \mathrm{e}^{\mathrm{i} k_{i}^{+} x}+B_{i}^{+}\left|\lambda_{i}^{+}\right\rangle \mathrm{e}^{-\mathrm{i} k_{i}^{+} x}+A_{i}^{-}\left|\lambda_{i}^{-}\right\rangle \mathrm{e}^{\mathrm{i} k_{i}^{-} x}+B_{i}^{-}\left|\lambda_{i}^{-}\right\rangle \mathrm{e}^{-\mathrm{i} k_{i}^{-} x}\right)
$$

with

$$
k_{i}^{ \pm}=\sqrt{k^{2}-2 m \lambda_{i}^{ \pm} / \hbar} .
$$

As in Section B.1 one can define matching matrices by

$$
\mathbf{N}_{i}(x)=\left[\begin{array}{cccc}
\mathrm{e}^{\mathrm{i} k_{i}^{+} x} & \mathrm{e}^{-\mathrm{i} k_{i}^{+} x} & \mathrm{e}^{\mathrm{i} k_{i}^{-} x} & \mathrm{e}^{-\mathrm{i} k_{i}^{-} x} \\
\mu_{i}^{+} \mathrm{e}^{\mathrm{i} k_{i}^{+} x} & \mu_{i}^{+} \mathrm{e}^{-\mathrm{i} k_{i}^{+} x} & \mu_{i}^{-} \mathrm{e}^{\mathrm{i} k_{i}^{-} x} & \mu_{i}^{-} \mathrm{e}^{-\mathrm{i} k_{i}^{-} x} \\
\mathrm{i} k_{i}^{+} \mathrm{e}^{\mathrm{i} k_{i}^{+} x} & -\mathrm{i} k_{i}^{+} \mathrm{e}^{-\mathrm{i} k_{i}^{+} x} & \mathrm{i} k_{i}^{-} \mathrm{e}^{\mathrm{i} k_{i}^{-} x} & -\mathrm{i} k_{i}^{-} \mathrm{e}^{-\mathrm{i} k_{i}^{-} x} \\
\mathrm{i} k_{i}^{+} \mu_{i}^{+} \mathrm{e}^{\mathrm{i} k_{i}^{+} x} & -\mathrm{i} k_{i}^{+} \mu_{i}^{+} \mathrm{e}^{-\mathrm{i} k_{i}^{+} x} & \mathrm{i} k_{i}^{-} \mu_{i}^{-} \mathrm{e}^{\mathrm{i} k_{i}^{-} x} & -\mathrm{i} k_{i}^{-} \mu_{i}^{-} \mathrm{e}^{-\mathrm{i} k_{i}^{-} x}
\end{array}\right]
$$

where $\mu_{i}^{ \pm}=2 \lambda_{i}^{ \pm} / \Omega_{i}$ has been defined for convenience. The matching conditions between sections with different Rabi frequencies take the form

$$
\mathbf{N}_{i}\left(x_{i+1}\right)\left[\begin{array}{c}
A_{i}^{+} \\
B_{i}^{+} \\
A_{i}^{-} \\
B_{i}^{-}
\end{array}\right]=\mathbf{N}_{i+1}\left(x_{i+1}\right)\left[\begin{array}{c}
A_{i+1}^{+} \\
B_{i+1}^{+} \\
A_{i+1}^{-} \\
B_{i+1}^{-}
\end{array}\right]
$$

and, similar to Eq. (B.6), the transfer matrix connecting the amplitudes of regions $i$ and $i+1$ is

$$
\mathbf{T}(i, i+1)=\mathbf{N}_{i}^{-1}\left(x_{i+1}\right) \mathbf{N}_{i+1}\left(x_{i+1}\right) .
$$


For $\Omega_{i}=0$, i.e. in a laser-free region, the general solution of the stationary Schrödinger equation is given by

$$
\boldsymbol{\Phi}_{k}(x)=\frac{1}{\sqrt{2 \pi}}\left(\begin{array}{c}
A_{i}^{+} \mathrm{e}^{\mathrm{i} k x}+B_{i}^{+} \mathrm{e}^{-\mathrm{i} k x} \\
A_{i}^{-} \mathrm{e}^{\mathrm{i} q x}+B_{i}^{-} \mathrm{e}^{-\mathrm{i} q x}
\end{array}\right),
$$

where $q=\sqrt{k^{2}+m(\mathrm{i} \gamma+2 \delta) / \hbar}$, which corresponds to the free matching matrix

$$
\mathbf{N}_{i, \text { free }}(x)=\left[\begin{array}{cccc}
\mathrm{e}^{\mathrm{i} k x} & \mathrm{e}^{-\mathrm{i} k x} & 0 & 0 \\
0 & 0 & \mathrm{e}^{\mathrm{i} q x} & \mathrm{e}^{-\mathrm{i} q x} \\
\mathrm{i} k \mathrm{e}^{\mathrm{i} k x} & -\mathrm{i} k \mathrm{e}^{-\mathrm{i} k x} & 0 & 0 \\
0 & 0 & \mathrm{i} q \mathrm{e}^{\mathrm{i} q x} & -\mathrm{i} q \mathrm{e}^{-\mathrm{i} q x}
\end{array}\right] .
$$

For matching conditions with a laser-free region, this matrix has to be used.

\section{B.2.1 Half-space laser field}

For a laser field localized in the region $x \geq 0$ (half-space laser field) there are two sections with $\Omega_{0}=0$ and $\Omega_{1} \equiv \Omega$, corresponding to a position-dependent Rabi frequency of the form $\Omega(x)=\Omega \Theta(x)$. With Eq. (B.31), the matching condition at $x_{1}=0$ reads

$$
\left[\begin{array}{c}
A_{0}^{+} \\
B_{0}^{+} \\
A_{0}^{-} \\
B_{0}^{-}
\end{array}\right]=\mathbf{T}(0,1)\left[\begin{array}{l}
A_{1}^{+} \\
B_{1}^{+} \\
A_{1}^{-} \\
B_{1}^{-}
\end{array}\right]
$$

where

$$
\mathbf{T}(0,1)=\mathbf{N}_{0, \text { free }}^{-1}(0) \mathbf{N}_{1}(0) .
$$

For a plane wave incoming from the left in the ground state one has $A_{0}^{+}=1$ and $A_{0}^{-}=B_{1}^{+}=B_{1}^{-}=0$ and the solution of Eq. (B.35) is given by

$$
\begin{aligned}
R_{1} \equiv B_{0}^{+} & =\frac{T_{23}(0,1) T_{31}(0,1)-T_{21}(0,1) T_{33}(0,1)}{T_{13}(0,1) T_{31}(0,1)-T_{11}(0,1) T_{33}(0,1)} \\
& =\left(\lambda^{+}\left(q+k^{+}\right)\left(k-k^{-}\right)-\lambda^{-}\left(q+k^{-}\right)\left(k-k^{+}\right)\right) / D \\
R_{2} \equiv B_{0}^{-} & =\frac{T_{43}(0,1) T_{31}(0,1)-T_{41}(0,1) T_{33}(0,1)}{T_{13}(0,1) T_{31}(0,1)-T_{11}(0,1) T_{33}(0,1)}=k\left(k^{-}-k^{+}\right) \Omega / D \\
C_{+} \equiv A_{1}^{+} & =\frac{-T_{33}(0,1)}{T_{13}(0,1) T_{31}(0,1)-T_{11}(0,1) T_{33}(0,1)}=-2 k\left(q+k^{-}\right) \lambda^{-} / D \\
C_{-} \equiv A_{1}^{-} & =\frac{T_{31}(0,1)}{T_{13}(0,1) T_{31}(0,1)-T_{11}(0,1) T_{33}(0,1)}=2 k\left(q+k^{+}\right) \lambda^{+} / D
\end{aligned}
$$

with the common denominator

$$
D=\left(k+k^{-}\right)\left(q+k^{+}\right) \lambda^{+}-\left(k+k^{+}\right)\left(q+k^{-}\right) \lambda^{-} .
$$




\section{B.2.2 Laser barrier}

For a laser barrier extending from $x=0$ to $x=L$ one has $\Omega(x)=\chi_{[0, L]}(x)$ and there are three sections, $i=1,2,3$. The transfer matrix connecting the laser-free sections 0 and 2 is given by

$$
\mathbf{T}(0,2)=\mathbf{N}_{0, \text { free }}^{-1}(0) \mathbf{N}_{1}(0) \mathbf{N}_{1}^{-1}(L) \mathbf{N}_{2, \text { free }}(L) .
$$

With $A_{0}^{+}=1$ and $A_{0}^{-}=B_{2}^{+}=B_{2}^{-}=0$, corresponding to a ground state wave incident from the left, the asymptotic reflection and transmission coefficients are given by the same matrix element expressions as in Eqs. (B.37)-(B.40), but for the matrix $\mathbf{T}(0,2)$. The amplitudes in the laser region are obtained by

$$
\left[\begin{array}{c}
A_{1}^{+} \\
B_{1}^{+} \\
A_{1}^{-} \\
B_{1}^{-}
\end{array}\right]=\mathbf{T}(0,1)^{-1}\left[\begin{array}{c}
1 \\
R_{1} \\
0 \\
R_{2}
\end{array}\right] .
$$

The arising expressions for the coefficients are not illuminating and thus not presented here, but they are helpful to discuss limits and approximations as it is done in the following sections.

\section{B.2.3 Dirac delta laser field}

In the limit of a very narrow, but strong laser field, $\Omega \sim L^{-1}, L \rightarrow 0$, the solution for the laser barrier equals the solution for a laser shape modeled by a Dirac delta function at position $x=0, \Omega(x)=\Omega L_{0} \delta(x)$, where $L_{0}$ is some arbitrary length. Applying this limit to the coefficients obtained in Section B.2.2 yields

$$
\begin{aligned}
R_{1} & =-\frac{m^{2} \Omega^{2} L_{0}^{2}}{4 \hbar^{2} k q+m^{2} \Omega^{2} L_{0}^{2}}, \\
R_{2}=T_{2} & =\frac{-2 \mathrm{i} \hbar m k \Omega L_{0}}{4 \hbar^{2} k q+m^{2} \Omega^{2} L_{0}^{2}}, \\
T_{1} & =\frac{4 \hbar^{2} k q}{4 \hbar^{2} k q+m^{2} \Omega^{2} L_{0}^{2}},
\end{aligned}
$$

and the stationary solution of the Schrödinger equation is given by

$$
\boldsymbol{\Phi}_{k}(x)=\frac{1}{\sqrt{2 \pi}} \begin{cases}\left(\begin{array}{c}
\mathrm{e}^{\mathrm{i} k x}+R_{1} \mathrm{e}^{-\mathrm{i} k x} \\
R_{2} \mathrm{e}^{-\mathrm{i} q x}
\end{array}\right), & x \leq 0 \\
\left(\begin{array}{l}
T_{1} \mathrm{e}^{\mathrm{i} k x} \\
T_{2} \mathrm{e}^{\mathrm{i} q x}
\end{array}\right), & x \geq 0 .\end{cases}
$$

The same expression is obtained, if one considers a delta-shape laser from the beginning and the corresponding matching conditions at $x=0$.

A very narrow laser beam is advantageous for the investigation of arrival times for wave packets with positive and negative momentum components, coming in from both sides. This is shown in Chapter 6 . 


\section{Appendix C}

\section{Faddeev-Marchenko inverse scattering methods}

In this chapter the inverse scattering methods which are used in the present work are summarized. For the inverse scattering problem on the line, various methods have been developed; an overview and further references are given in Refs. [116, 139]. Here the Faddeev-Marchenko method shall be used [116]. A comprehensive introduction with numerous examples can be found in Ref. [118].

\section{C.1 One-dimensional scattering}

In the following the one-dimensional stationary Schrödinger equation in atomic units is considered,

$$
\phi_{k}^{\prime \prime}(x)+k^{2} \phi_{k}(x)=2 U(x) \phi_{k}(x)
$$

where $U(x)$ is a real-valued potential belonging to the class $L_{1}^{1}(\mathbb{R})$ of measurable potentials such that

$$
\int_{-\infty}^{\infty} \mathrm{d} x(1+|x|)|U(x)|<\infty
$$

The direct scattering problem is the problem of finding appropriate solutions to Eq. (C.1) for $k \in \mathbb{R}$ that can be used to describe the scattering process associated with the time-dependent Schrödinger equation. The solution of Eq. (C.1) allows to identify a set of data, the scattering data, that describes characteristic features of the scattering process, such as transmission or reflection. The inverse scattering problem, on the other hand, deals with the construction of $U(x)$ using the scattering data.

For a plane wave incident from the left, the solutions of Eq. (C.1) take the asymptotic form

$$
\begin{aligned}
& \phi_{k}(x) \sim \mathrm{e}^{\mathrm{i} k x}+R_{\mathrm{L}}(k) \mathrm{e}^{-\mathrm{i} k x}, \quad x \rightarrow-\infty, \\
& \phi_{k}(x) \sim T(k) \mathrm{e}^{\mathrm{i} k x}, \quad x \rightarrow \infty,
\end{aligned}
$$


whereas for a plane wave incoming from the right one has

$$
\begin{aligned}
& \phi_{k}(x) \sim T(k) \mathrm{e}^{-\mathrm{i} k x}, \quad x \rightarrow-\infty, \\
& \phi_{k}(x) \sim \mathrm{e}^{-\mathrm{i} k x}+R_{\mathrm{R}}(k) \mathrm{e}^{\mathrm{i} k x}, \quad x \rightarrow \infty,
\end{aligned}
$$

where $T(k)$ is the transmission coefficient and $R_{\mathrm{L}}(k)\left(R_{\mathrm{R}}(k)\right)$ is the reflection coefficient for incidence from the left (right). The following relations between these scattering amplitudes hold for real-valued potentials and $k \in \mathbb{R}$ :

$$
\begin{aligned}
& |T(k)|^{2}+\left|R_{\mathrm{L}}(k)\right|^{2}=|T(k)|^{2}+\left|R_{\mathrm{R}}(k)\right|^{2}=1, \\
& T(-k)=\overline{T(k)}, \quad R_{\mathrm{L}}(-k)=\overline{R_{\mathrm{R}}(k)}, \quad R_{\mathrm{R}}(-k)=\overline{R_{\mathrm{R}}(k)} \\
& R_{\mathrm{R}}(k) \overline{T(k)}=-\overline{R_{\mathrm{L}}(k)} T(k) .
\end{aligned}
$$

Bound states: In addition to the scattering solutions, Eq. (C.1) may have normalizable solutions for $k^{2}<0$, which are referred to as bound states. If $V \in L_{1}^{1}(\mathbb{R})$, then $T(k)$ is meromorphic in the upper-half complex plane and the number of its poles is finite; each of these poles is simple, occurs on the positive imaginary axis, and corresponds to a bound state of $U(x)$ [139].

\section{C.2 Relation between transmission and reflection coefficients}

It is obvious from Eqs. (C.7)-(C.9) that some redundancy is incorporated in the scattering data. It can be shown that all of the coefficients $T(k), R_{\mathrm{L}}(k), R_{\mathrm{R}}(k)$ can be constructed in terms of the bound-state energies and either one of the reflection coefficients. If the potential contains no bound states and $T(k)$ has no pole in the upper-half complex plane, the following relation holds [117]:

$$
\frac{\mathrm{d}}{\mathrm{d} k} \ln T(k)=-\frac{1}{2 \pi \mathrm{i}} \int_{-\infty}^{\infty} \mathrm{d} \xi \frac{\frac{\mathrm{d}}{\mathrm{d} \xi}|R(\xi)|^{2}}{\left(\xi-k-\mathrm{i} 0^{+}\right)\left(1-|R(\xi)|^{2}\right)},
$$

where either $R_{\mathrm{L}}(k)$ or $R_{\mathrm{R}}(k)$ may be inserted due to Eq. (C.7). The integration constant is fixed by $T(k) \rightarrow 1$ for $k \rightarrow \infty$. The integral is particularly easy to evaluate when $R(k)$ is a rational function of $k$.

If $T(k)$ contains first-order poles in the upper-half complex plane at points $\mathrm{i} \lambda_{j}$, Eq. (C.10) holds for the new function

$$
\tilde{T}(k)=T(k) \prod_{j} \frac{k-\mathrm{i} \lambda_{j}}{k+\mathrm{i} \lambda_{j}}
$$

which has now the properties required above for $T(k)$. 


\section{C.3 Marchenko equations without bound states}

In this section, the necessary relations for the determination of the potential $U(x)$ within the Faddeev-Marchenko approach are given without proof. A detailed analysis may be found in Refs. [118, 116].

For later purpose, the Fourier transforms of the left and the right reflection coefficients are defined by

$$
\begin{aligned}
& r_{\mathrm{L}}(z)=\frac{1}{2 \pi} \int_{-\infty}^{\infty} \mathrm{d} k \mathrm{e}^{-\mathrm{i} k z} R_{\mathrm{L}}(k), \\
& r_{\mathrm{R}}(z)=\frac{1}{2 \pi} \int_{-\infty}^{\infty} \mathrm{d} k \mathrm{e}^{\mathrm{i} k z} R_{\mathrm{R}}(k) .
\end{aligned}
$$

They determine the left and the right Marchenko equations, which are integral equations for unknown functions $A_{\mathrm{L}}(x, y)$ and $A_{\mathrm{R}}(x, y)$ and take the following form:

$$
\begin{array}{ll}
0=r_{\mathrm{L}}(x+y)+A_{\mathrm{L}}(x, y)+\int_{-\infty}^{x} \mathrm{~d} x^{\prime} r_{\mathrm{L}}\left(x^{\prime}+y\right) A_{\mathrm{L}}\left(x, x^{\prime}\right), & x>y, \\
0=r_{\mathrm{R}}(x+y)+A_{\mathrm{R}}(x, y)+\int_{x}^{\infty} \mathrm{d} x^{\prime} r_{\mathrm{R}}\left(x^{\prime}+y\right) A_{\mathrm{R}}\left(x, x^{\prime}\right), \quad x<y .
\end{array}
$$

The desired potential $U(x)$ is given by the functions $A_{\mathrm{L}}(x, y)$ and $A_{\mathrm{R}}(x, y)$ through the relations

$$
\begin{aligned}
& U(x)=-\frac{\mathrm{d}}{\mathrm{d} x} \lim _{\epsilon \rightarrow 0^{+}} A_{\mathrm{R}}(x, x+\epsilon), \\
& U(x)=\frac{\mathrm{d}}{\mathrm{d} x} \lim _{\epsilon \rightarrow 0^{+}} A_{\mathrm{L}}(x, x-\epsilon) .
\end{aligned}
$$

\section{C.4 Marchenko equations with bound states}

In the presence of bound states, the derivation of the potential $U(x)$ is more complicated. Assuming the poles of the transmission coefficient $T(k)$ in the upper-half space to be at $k=\mathrm{i} \lambda_{j}$, new functions $s_{\mathrm{L}}(z)$ and $s_{\mathrm{R}}(z)$ have to be defined by:

$$
\begin{aligned}
& s_{\mathrm{L}}(z)=r_{\mathrm{L}}(z)-\mathrm{i} \sum_{j} \operatorname{Res}_{\mathrm{i} \lambda_{j}}[T(k)] \frac{R_{\mathrm{L}}\left(\mathrm{i} \lambda_{j}\right)}{T\left(\mathrm{i} \lambda_{j}\right)} \mathrm{e}^{\lambda_{j} z}, \\
& s_{\mathrm{R}}(z)=r_{\mathrm{R}}(z)-\mathrm{i} \sum_{j} \operatorname{Res}_{\mathrm{i} \lambda_{j}}[T(k)] \frac{R_{\mathrm{R}}\left(\mathrm{i} \lambda_{j}\right)}{T\left(\mathrm{i} \lambda_{j}\right)} \mathrm{e}^{-\lambda_{j} z} .
\end{aligned}
$$

Now the Marchenko equations for the functions $A_{\mathrm{L}}(x, y)$ and $A_{\mathrm{R}}(x, y)$ are exactly the same integral equations as in the case of no bound states where $r_{\mathrm{L}}(z)$ and $r_{\mathrm{R}}(z)$ are replaced by $s_{\mathrm{L}}(z)$ and $s_{\mathrm{R}}(z)$, respectively:

$$
\begin{array}{ll}
0=s_{\mathrm{L}}(x+y)+A_{\mathrm{L}}(x, y)+\int_{-\infty}^{x} \mathrm{~d} x^{\prime} s_{\mathrm{L}}\left(x^{\prime}+y\right) A_{\mathrm{L}}\left(x, x^{\prime}\right), & x>y, \\
0=s_{\mathrm{R}}(x+y)+A_{\mathrm{R}}(x, y)+\int_{x}^{\infty} \mathrm{d} x^{\prime} s_{\mathrm{R}}\left(x^{\prime}+y\right) A_{\mathrm{R}}\left(x, x^{\prime}\right), & x<y .
\end{array}
$$

For the determination of the potential, Eqs. (C.16) and (C.17) hold as before. 


\section{Appendix D}

\section{Atomic units}

For convenience, numbers are given in specific atomic units in this work. They are defined in the following. Additionally, a list of useful conversion coefficients is provided.

\section{Definition (atomic units):}

$$
\begin{aligned}
\hbar & =1 \\
1 \text { unit of mass } & =m_{\mathrm{Cs}}=2.2 \times 10^{-25} \mathrm{~kg} \\
1 \text { unit of time } & =10^{-6} \mathrm{~s} .
\end{aligned}
$$

This uniquely fixes all other units. For instance, one has

$$
\begin{aligned}
1 \text { unit of length } & =0.0213 \mu \mathrm{m} \\
1 \text { unit of frequency } & =10^{6} \mathrm{~s}^{-1} \\
1 \text { unit of velocity } & =2.13 \mathrm{~cm} / \mathrm{s} \\
1 \text { unit of energy } & =10^{6} \hbar \mathrm{s}^{-1} \\
1 \text { unit of momentum } & =4.69 \times 10^{-27} \mathrm{~kg} \mathrm{~m} / \mathrm{s} .
\end{aligned}
$$

For the optical transition used in this work, the transition $6^{2} P_{3 / 2}-6^{2} S_{1 / 2}$ of cesium is considered. The Einstein coefficient for this transition is $\gamma=33.3 \times 10^{6} \mathrm{~s}^{-1}$ [24]. 


\section{Bibliography}

[1] J.G. Muga, R. Sala, I.L. Egusquiza (eds.), Time in Quantum Mechanics (Springer, Berlin, 2002).

[2] D.J. Auerbach, in: Atomic and Molecular Beam Methods, edited by G. Scoles (Oxford University Press, New York, 1988), chap. 14, pp. 362-379.

[3] P. Szriftgiser, D. Guéry-Odelin, M. Arndt, and J. Dalibard, Phys. Rev. Lett. 77, 4 (1996).

[4] J.G. Muga and G.R. Leavens, Phys. Rep. 338, 353 (2000).

[5] W. Pauli, in: Handbuch für Physik, edited by H. Geiger and K. Scheel (Springer, Berlin, 1933), chap. XXIV, p. 140.

[6] G.R. Allcock, Ann. Phys. (N.Y.) 53, 253;286;311 (1969).

[7] J.G. Muga, S. Brouard, and D. Macias, Ann. Phys. (N.Y.) 240, 351 (1995).

[8] Y. Aharonov, J. Oppenheim, S. Popescu, B. Reznik, and W.G. Unruh, Phys. Rev. A 57, 4130 (1998).

[9] Y. Aharonov and D. Bohm, Phys. Rev. 122, 1649 (1961).

[10] J. Kijowski, Rep. Math. Phys. 6, 361 (1974).

[11] R. Giannitrapani, Int. J. Theor. Phys. 36, 1575 (1997).

[12] P. Busch, M. Grabowski, and P.J. Lahti, Operational Quantum Mechanics (Springer, Berlin, 1995).

[13] E.H. Hauge and J.A. Støvneng, Rev. Mod. Phys. 61, 917 (1989).

[14] R. Landauer and T. Martin, Rev. Mod. Phys. 66, 217 (1994).

[15] G.C. Hegerfeldt, in: Extensions of Quantum Theory 3, edited by A. Horzela and E. Kapuscik (Apeiron, Montreal, 2001), p. 9.

[16] G. Nimtz, A. Enders, and H. Spieker, J. Phys. I France 4, 565 (1994).

[17] A.M. Steinberg and R.Y. Chiao, Phys. Rev. A 51, 3525 (1995). 
[18] T. Hartman, J. Appl. Phys. 33, 3427 (1962).

[19] E. Wigner, Phys. Rev. 98, 145 (1955).

[20] C.R. Leavens and G.C. Aers, Phys. Rev. B 40, 5387 (1989).

[21] C.R. Leavens and R. Sala Mayato, Ann. Phys. (Leipzig) 7, 662 (1998).

[22] J. Halliwell, Progr. Theor. Phys. 102, 707 (1999).

[23] J.G. Muga, A.D. Baute, J.A. Damborenea, and I.L. Egusquiza, quant$\mathrm{ph} / 0009111$ (2000).

[24] J.A. Damborenea, I.L. Egusquiza, G.C. Hegerfeldt, and J.G. Muga, Phys. Rev. A 66, 052104 (2002).

[25] A.D. Baute, I.L. Egusquiza, and J.G. Muga, Phys. Rev. A 64, 012501 (2001).

[26] H. Ekstein and A.J.F. Siegert, Ann. Phys. 68, 509 (1971).

[27] R. Brunetti and K. Fredenhagen, Phys. Rev. A 66, 044101 (2002).

[28] N. Grot, C. Rovelli, and R.S. Tate, Phys. Rev. A 54, 4676 (1996).

[29] M. Razavy, Can. J. Phys. 49, 3075 (1971).

[30] V. Delgado and J.G. Muga, Phys. Rev. A 56, 3425 (1997).

[31] E.A. Galapon, Proc. R. Soc. Lond. A 458, 451 (2002).

[32] E.A. Galapon, R.F. Caballar, and R.T. Bahague, Phys. Rev. Lett. 93, 180406 (2004).

[33] J. Kijowski, Phys. Rev. A 59, 897 (1999).

[34] R. Werner, Ann. Inst. Henri Poincare 47, 429 (1987).

[35] N. Yamada and S. Takagi, Prog. Theor. Phys. 85, 985 (1991).

[36] N. Yamada and S. Takagi, Prog. Theor. Phys. 86, 599 (1991).

[37] N. Yamada and S. Takagi, Prog. Theor. Phys. 87, 77 (1992).

[38] P. Kochański and K. Wódkiewicz, Phys. Rev. A 60, 2689 (1999).

[39] C. Leavens, Phys. Rev. A 58, 840 (1998).

[40] C. Leavens, Phys. Lett. A 303, 154 (2002).

[41] J. Finkelstein, Phys. Rev. A 59, 3218 (1999).

[42] J. Ruseckas and B. Kaulakys, Phys. Lett. A 287, 297 (2001). 
[43] J. Ruseckas and B. Kaulakys, Phys. Rev. A 66, 052106 (2002).

[44] J.G. Muga, C.R. Leavens, and J.P. Palao, Phys. Rev. A 58, 4336 (1998).

[45] M. Toller, Phys. Rev. A 59, 960 (1999).

[46] V. Delgado, Phys. Rev. A 59, 1010 (1999).

[47] I.L. Egusquiza and J.G. Muga, Phys. Rev. A 61, 012104 (2000).

[48] A.J. Bracken and G.F. Melloy, J. Phys. A: Math. Gen. 27, 2197 (1994).

[49] R. Werner, J. Math. Phys. 27, 793 (1986).

[50] J. León, J. Julve, P. Pitanga, and F.J. de Urries, Phys. Rev. A 61, 062101 (2000).

[51] L. Cohen and P. Loughlin, J. Mod. Opt. 49, 539 (2002).

[52] J.G. Muga, J.P. Palao, and R. Sala, Phys. Lett. A 238, 90 (1998).

[53] P. Guéret, A. Baratoff, and E. Marclay, Europhys. Lett. 3, 367 (1987).

[54] A.M. Steinberg, P.G. Kwiat, and R.Y. Chiao, Phys. Rev. Lett. 71, 708 (1993).

[55] P. Balcou and L. Dutriaux, Phys. Rev. Lett. 78, 851 (1997).

[56] D. Mugnai and A. Ranfagni, in: Time in Quantum Mechanics, edited by J.G. Muga, R. Sala Mayato and I.L. Egusquiza (Springer, Berlin, 2002), chap. 12 , pp. 326-368.

[57] V.S. Olkhovsky and E. Recami, Phys. Rep. 214, 339 (1992).

[58] J. Fletcher, J. Phys. C: Solid State Phys. 18, L55 (1985).

[59] F. Raciti and G. Salesi, J. Phys. I France 4, 1783 (1994).

[60] M. Sassoli de Bianchi, Eur. J. Phys. 21, L21 (2000).

[61] M. Büttiker and R. Landauer, Phys. Rev. Lett. 49, 1739 (1982).

[62] J.G. Muga, S. Brouard, and R. Sala, Phys. Lett. A 167, 24 (1992).

[63] V. Delgado, S. Brouard, and J.G. Muga, Solid State Commun. 94, 979 (1995).

[64] S. Brouard, R. Sala, and J.G. Muga, Phys. Rev. A 49, 1994 (1994).

[65] E. Pollak and W.H. Miller, Phys. Rev. Lett. 53, 115 (1984).

[66] F. Smith, Phys. Rev. 118, 349 (1960).

[67] M. Büttiker, Phys. Rev. B 27, 6178 (1983). 
[68] A. Baz', Sov. J. Nucl. Phys. 4, 182 (1967).

[69] V. Rybachenko, Sov. J. Nucl. Phys. 5, 635 (1967).

[70] D. Sokolovski and L.M. Baskin, Phys. Rev. A 36, 4604 (1987).

[71] H. Fertig, Phys. Rev. Lett. 65, 2321 (1990).

[72] Z.S. Wang, L.C. Kwek, C.H. Lai, and C.H. Oh, Phys. Rev. A 69, 052108 (2004).

[73] W. Jaworski and D.M. Wardlaw, Phys. Rev. A 37, 2843 (1988).

[74] W. Jaworski, J. Math. Phys. 30, 1505 (1989).

[75] W. Jaworski and D.M. Wardlaw, Phys. Rev. A 40, 6210 (1989).

[76] J.A. Damborenea, I.L. Egusquiza, J.G. Muga, and B. Navarro, quantph/0403081 (2004).

[77] D. Alonso, R. Sala Mayato, and J.G. Muga, Phys. Rev. A 67, 032105 (2003).

[78] R. Golub, S. Felber, R. Gähler, and E. Gutsmiedl, Phys. Lett. A 148, 27 (1990).

[79] P. Blanchard and A. Jadczyk, Phys. Lett. A 175, 157 (1993).

[80] A. Jadczyk, Progr. Theor. Phys. 93, 631 (1995).

[81] J.A. Damborenea, I.L. Egusquiza, G.C. Hegerfeldt, and J.G. Muga, J. Phys. B: At. Mol. Opt. Phys. 36, 2657.

[82] B. Navarro, I.L. Egusquiza, J.G. Muga, and G.C. Hegerfeldt, J. Phys. B: At. Mol. Opt. Phys. 36, 3899 (2003).

[83] B. Navarro, I.L. Egusquiza, J.G. Muga, and G.C. Hegerfeldt, Phys. Rev. A 67, 063819 (2003).

[84] G.C. Hegerfeldt, D. Seidel, and J.G. Muga, Phys. Rev. A 68, 022111 (2003).

[85] G.C. Hegerfedt and T.S. Wilser, in: Classical and Quantum Systems. Proceedings of the Second International Wigner Symposium 1991, edited by H.D. Doebner, W. Scherer and F. Schroeck (World Scientific, Singapore, 1991), p. 104.

[86] G.C. Hegerfeldt, Phys. Rev. A 47, 449 (1993).

[87] G.C. Hegerfeldt, in: Springer LNP 622, Irreversible Quantum Dynamics, edited by F. Benatti and R. Floreanini (Springer, 2003), p. 233.

[88] J. Dalibard, Y. Castin, and K. Mølmer, Phys. Rev. A 68, 580 (1992). 
[89] H. Carmichael, An Open Systems Approach to Quantum Optics, Lecture Notes in Physics (Springer, Berlin, 1993).

[90] M.B. Plenio and P.L. Knight, Rev. Mod. Phys. 70, 101 (1998).

[91] The projection postulate as commonly used nowadays is due to G. Lüders, Ann. Phys. 8, 323 (1951). For observables with degenerate eigenvalues his formulation differs from that of J. von Neumann, Mathematische Grundlagen der Quantenmechanik, (Springer, Berlin, 1932).

[92] G.S. Agarwal, Quantum Optics, Springer Tracts of Modern Physics, Vol. 70 (Springer, Berlin, 1974).

[93] D. Müller, D.Z. Anderson, R.J. Grow, P.D.D. Schwindt, and E.A. Cornell, Phys. Rev. Lett. 83, 5194 (1999).

[94] D. Schneble, M. Hasuo, T. Anker, T. Pfau, and J. Mlynek, J. Opt. Soc. Am. B 20, 648 (2003).

[95] V. Hannstein, G.C. Hegerfeldt, and J.G. Muga, J. Phys. B: At. Mol. Opt. Phys. 38, 409 (2005).

[96] M.S. Kim, P.L. Knight, and K. Wódkiewicz, Opt. Commun. 62, 385 (1987).

[97] L.I. Schiff, Quantum Mechanics (McGraw-Hill, New York, 1955), chap. II, p. 24.

[98] G.C. Hegerfeldt, unpublished.

[99] J.G. Muga, J.P. Palao, B. Navarro, and I.L. Egusquiza, Phys. Rep. 395, 357 (2004).

[100] J.G. Muga and B. Navarro, Chem. Phys. Lett. 390, 454 (2004).

[101] H. Feshbach, Ann. Phys. (N.Y.) 5, 357 (1958).

[102] H. Feshbach, Ann. Phys. (N.Y.) 19, 287 (1958).

[103] A. Ruschhaupt, J.A. Damborenea, B. Navarro, J.G. Muga, and G.C. Hegerfeldt, Europhys. Lett. 67, 1 (2004).

[104] E.H. Lieb, Rev. Mod. Phys. 53, 603 (1981).

[105] P.W. Ayers, R.G. Parr, and A. Nagy, Int. J. Quant. Chem. 90, 309 (2002).

[106] R.F.W. Bader and P.M. Beddall, J. Chem. Phys. 56, 3320 (1972).

[107] L. Cohen, J. Chem. Phys. 70, 788 (1979).

[108] A. Tachibana, J. Chem. Phys. 115, 3497 (2001).

[109] D.C. Rivier, Phys. Rev. 83, 862 (1957). 
[110] Y. Aharonov, S. Popescu, D. Rohrlich, and L. Vaidman, Phys. Rev. A 48, 4084 (1993).

[111] L.M. Johansen, Phys. Rev. Lett. 93, 120402 (2004).

[112] L.M. Johansen, quant-ph/9804002 (1998).

[113] J.G. Muga, D. Seidel, and G.C. Hegerfeldt, J. Chem. Phys. 122, 154106 (2005).

[114] Y.Z. Huang and C.M. Wang, J. Phys.: Condens. Matt. 3, 5915 (1991).

[115] J.P. Palao, J.G. Muga, S. Brouard, and A. Jadczyk, Phys. Lett. A 233, 227 (1997).

[116] K. Chadan and P. Sabatier, Inverse Problems in Quantum Scattering Theory (Springer, New York, 1989).

[117] I. Kay, Commun. Pure Appl. Math. 13, 371 (1960).

[118] G. Lamb, Elements of soliton theory (John Wiley \& Sons, New York, 1980).

[119] R.S. Dumont and T.L. Marchioro II, Phys. Rev. A 47, 85 (1993).

[120] K.I. Aoki, A. Horikoshi, and E. Nakamura, Phys. Rev. A 62, 022101 (2000).

[121] E. Nelson, Phys. Rev. 150, 1079 (1966).

[122] G.C. Hegerfeldt, D. Seidel, J.G. Muga, and B. Navarro, Phys. Rev. A 70, 012110 (2004).

[123] G.S. Agarwal and R. Arun, Phys. Rev. Lett. 84, 5098 (2000).

[124] R. Arun and G.S. Agarwal, Phys. Rev. A 64, 065802 (2001).

[125] J.G. Muga, I.L. Egusquiza, J.A. Damborenea, and F. Delgado, Phys. Rev. A 66, 042115 (2002).

[126] H. Winful, Phys. Rev. Lett. 91, 260401 (2003).

[127] W. van Dijk and K.A. Kiers, Am. J. Phys. 60, 520 (1992).

[128] C.J. Bordé, Metrologia 39, 435 (2002).

[129] M. Abramowitz, I.A. Stegun (eds.), Handbook of mathematical functions, (Dover Publications, New York, 1968).

[130] R.E. Crandall and B.R. Litt, Ann. Phys. (NY) 146, 458 (1983).

[131] R.L. Jaffe, An Algebraic Approach to Reflectionless Potentials in One Dimension, unpublished. 
[132] R.K. Dodd, J.C. Eilbeck, J.D. Gibbon, and H.C. Morris, Solitons and Nonlinear Wave Equations (Academic Press, London, 1982), p. 71.

[133] M. Sassoli de Bianchi, J. Math. Phys. 35, 2719 (1994).

[134] S.H. Dong, Z.Q. Ma, and M. Klaus, quant-ph/9903016 (1999).

[135] F. Delgado, J.G. Muga, and A. Ruschhaupt, Phys. Rev. A 69, 022106 (2004).

[136] E. Merzbacher, Quantum Mechanics (Wiley, New York, 1961), p. 92.

[137] M. Razavy, Quantum theory of tunneling (World Scientific, Singapore, 2003).

[138] M.G. Rozman, P. Reineker, and R. Tehver, Phys. Rev. A 49, 3310 (1994).

[139] T. Aktosun and M. Klaus, in: Scattering, edited by E.R. Pike and P.C. Sabatier (Academic Press, London, 2001), chap. 2.2.4, pp. 770-785. 




\section{Danksagung}

Hiermit möchte ich mich bei all denen bedanken, die mir während der Promotionszeit mit Rat und Tat zur Seite standen und die zum Gelingen dieser Arbeit beigetragen haben.

An erster Stelle danke ich Herrn Prof. Dr. Gerhard C. Hegerfeldt für seine gute Betreuung, für die interessante Themenstellung sowie für die zahlreichen Möglichkeiten, die Ergebnisse meiner Arbeit zu präsentieren. Herrn Prof. Dr. Kurt Schönhammer danke ich für die Übernahme des Korreferates.

Weiterhin danke ich Volker Hannstein für eine schöne gemeinsame Zeit im Büro sowie Volker Hannstein und Jens-Timo Neumann für viele hilfreiche fachliche Diskussionen. Volker Hannstein, Lucie Hamdi sowie Maike Dittmar bin ich sehr verbunden für die undankbare Arbeit des Korrekturlesens.

Meiner liebsten Maike danke ich für ihr stets offenes Ohr für Probleme aller Art und für noch viel mehr. Nicht zuletzt geht mein Dank an meine Eltern und meinen Onkel für ihre ständige Unterstützung während meiner Promotionszeit.

Die Finanzierung meiner Promotion wurde durch ein Georg-Christoph-Lichtenberg-Stipendium des Landes Niedersachsen im Rahmen der Göttingen Graduate School of Physics getragen.

\section{Acknowledgements}

I gratefully acknowlegde many helpful discussions with Prof. J. Gonzalo Muga from the University of the Basque Country, Bilbao.

Moreover, I would like to thank Prof. J. Gonzalo Muga, Iñigo L. Egusquiza, Beatriz Navarro, Fernando Delgado, José P. Palao and Rafael Sala Mayato for their hospitality during workshops and for a great time in Bilbao and in Tenerife - muchas gracias! 



\title{
Lebenslauf
}

\author{
Name: $\quad$ Dirk Seidel \\ Geburtstag: $\quad$ 4. Mai 1977 \\ Geburtsort: Halle(Saale) \\ Staatsangehörigkeit: deutsch
}

\section{Wissenschaftlicher Werdegang:}

1983 - $1991 \quad$ Grundschule "POS Frohe Zukunft", Halle(Saale)

1991 - 1995 Gymnasium "Latina August-Hermann Francke", Halle(Saale), Abitur 1995

10/1995 - 09/1996 Zivildienst, Diakonie Halle(Saale)

10/1996-9/1998 Physikstudium, Martin-Luther-Universität Halle(Saale), Vordiplom 1998

10/1998 - 11/2001 Physikstudium, Georg-August-Universität Göttingen, Diplomarbeit am Institut für Theoretische Physik in der Arbeitsgruppe von Prof. G.C. Hegerfeldt, Diplom 11/2001

3/2002 - 5/2002 Wissenschaftliche Hilfskraft am Institut für Theoretische Physik in Göttingen

5/2002-7/2005 Promotionsstudent an der Universität Göttingen, GeorgChristoph-Lichtenberg-Stipendium des Landes Niedersachsen, Promotion am Institut für Theoretische Physik in der Arbeitsgruppe von Prof. G.C. Hegerfeldt 